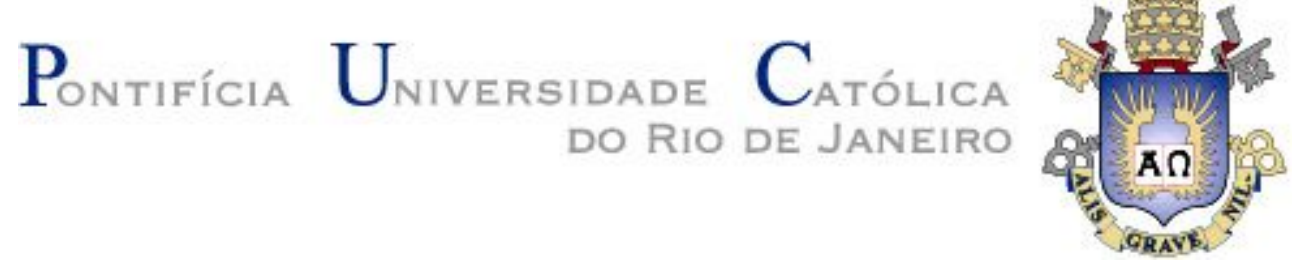

Edgar Sarmiento Calisaya

Analysis of Natural Language Scenarios

Thesis presented to the Programa de Pós-graduação em Informática, of the Departamento de Informática do Centro Técnico Científico da PUC-Rio, as partial fulfillment of the requirements for the degree of Doutor.

Advisor: Prof. Julio Cesar Sampaio do Prado Leite

Rio de Janeiro

April 2016 


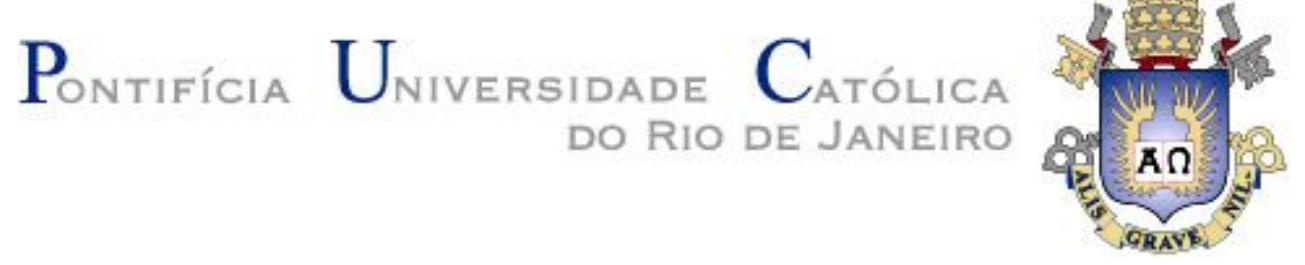

Edgar Sarmiento Calisaya

\section{Analysis of Natural Language Scenarios}

Thesis presented to the Programa de Pós-Graduação em Informática, of the Departamento de Informática do Centro Técnico Científico da PUC-Rio, as partial fulfillment of the requirements for the degree of Doutor.

Prof. Julio Cesar Sampaio do Prado Leite Advisor

Departamento de Informática - PUC-Rio

Prof. Arndt von Staa

Departamento de Informática - PUC-Rio

Prof. Carlos José Pereira de Lucena Departamento de Informática - PUC-Rio

Prof. Marcos Roberto da Silva Borges

UFRJ

Prof. Eduardo Kinder Almentero

UFRRJ

Prof. Vera Maria B. Werneck

UERJ

Prof. Márcio da Silveira Carvalho

Coordinator of the Centro Técnico Científico da PUC-Rio

Rio de Janeiro, April 13th, 2016. 
All rights reserved

\section{Edgar Sarmiento Calisaya}

Graduated in Systems Engineering (Computer Science) from Universidad Nacional de San Agustin - Arequipa - Perú. He obtained the degree of Master in Informatics at Universidade federal do Rio de Janeiro - Rio de janeiro - Brazil. He has been working in the field of Software Engineering for over fifteen years.

Ficha Catalográfica

Sarmiento Calisaya, Edgar

Analysis of Natural Language Scenarios / Edgar Sarmiento Calisaya ; advisor: Julio Cesar Sampaio do Prado Leite. - 2016. $231 \mathrm{f}$ : : il. ; $30 \mathrm{~cm}$

Tese (doutorado)-Pontifícia Universidade Católica do Rio de Janeiro, Departamento de Informática, 2016.

Inclui bibliografia

1. Informática - Teses. 2. Requisitos de software. 3. Cenários. 4. Petri-Net. 5. Análise de requisitos. 6. Verificação de requisitos. I. Leite, Julio Cesar Sampaio do Prado. II. Pontifícia Universidade Católica do Rio de Janeiro. Departamento de Informática. III. Título. 


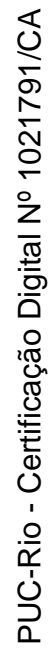

To my parents, Antonia Calisaya Sarmiento and Fabio Estanislao Sarmiento

Choque. 


\section{Acknowledgements}

First of all, I would like to give my most sincere tribute and gratitude to my advisor Julio Cesar Sampaio do Prado Leite who believed in this work. Their academic vision and timely discussions always inspire me. For his friendship, guidance, encouragement and insights, which guide me through my Ph.D life.

Futhermore, I would like to thank my parents Antonia and Fabio Estanislao for education and caring, for giving me all the necessary support, so that I could come here and be able to do this Ph.D study.

I would like to thank my brothers Lourdes, Juan Estanislao and Alberto for their companionship, love and patience in listening to me every time I needed.

I would like to thank to the professors Vera Maria B. Werneck, Arndt von Staa, Noemi Rodrigues and Marcos Roberto da Silva Borges for their important contributions; and who participated in the examination Committee.

To my mates Roxana, Giovana, Elizabeth, Joanna, Priscilla, Marilia, Andre, Eduardo and Henrique of Requirements Engineering research group at PUC-Rio, for the inspiration, knowledge, contributions, fellowship and time spend on the problem presented in this thesis.

Additionally, I am grateful to my friends Guina, Ruben Rafael, Giovana, Roxana, Fernanda and Gilbert, who were always very helpful and supportive.

I am also thankful to professors at PUC-Rio for everything I learned from them.

In adittion, I would like to thank to CAPES funding agency and the PUC-Rio, for the financial support. 


\section{Abstract}

Sarmiento Calisaya, Edgar; Leite, Julio Cesar Sampaio do Prado. Analysis of Natural Language Scenarios. Rio de Janeiro, 2016, 231p. DSc Thesis Departamento de informática, Pontifícia Universidade Católica do Rio de Janeiro.

Requirements analysis plays a key role in the software development process. Natural language-based scenario representations are often used for writing software requirements specifications (SRS). Scenarios written using natural language may be ambiguous, and, sometimes, inaccurate. This problem is partially due to the fact that relationships among scenarios are rarely represented explicitly. As scenarios are used as input to subsequent activities of the software development process (SD), it is very important to enable their analysis; especially to detect defects due to wrong information or missing information. This work proposes a Petri-Net and Natural Language Processing (NLP) based approach as an effective way to analyze the acquired scenarios, which takes textual description of scenarios (conform to a metamodel defined in this work) as input and generates an analysis report as output. To enable the automated analysis, scenarios are translated into equivalent Place/Transition Petri-Nets. Scenarios and their resulting Petri-Nets can be automatically analyzed to evaluate some properties related to unambiguity, completeness, consistency and correctness. The identified defects can be traced back to the scenarios, allowing their revision. We also discuss how unambiguity, completeness, consistency and correctness of scenariobased SRSs can be decomposed in related properties, and define heuristics for searching defect indicators that hurt these properties. We evaluate our work by applying our analysis approach to four case studies. The evaluation compares the results achieved by our tool-supported approach, with an inspection based approach and with related work.

\section{Keywords}

Software requirements; scenarios; use cases; requirements analysis; requirements verification; Petri-Net; Natural Language Processing. 


\section{Resumo}

Sarmiento Calisaya, Edgar; Leite, Julio Cesar Sampaio do Prado. Análise de Cenários em Linguagem Natural. Rio de Janeiro, 2016, 231p. Tese de Doutorado - Departamento de informática, Pontifícia Universidade Católica do Rio de Janeiro.

A análise de requisitos desempenha um papel fundamental no processo de desenvolvimento de software. Neste sentido, representações de cenários baseados em linguagem natural são muitas vezes utilizados para descrever especificações de requisitos de software (SRS). Cenários descritos usando linguagem natural podem ser ambíguos e, às vezes, imprecisos. Este problema é parcialmente devido ao fato de que os relacionamentos entre os cenários são raramente representados explicitamente. Como os cenários são utilizados como entrada para as actividades subsequentes do processo de desenvolvimento de software (SD), é muito importante facilitar a sua análise; especialmente para detectar defeitos devido a informações erradas ou falta de informação. Este trabalho propõe uma abordagem baseada em Redes de Petri e técnicas de Processamento de Linguagem Natural como uma forma eficaz para analisar os cenários adquiridos, e que toma descrições textuais de cenários (em conformidade com um metamodelo definido neste trabalho) como entrada e gera um relatório de análise como saída. Para facilitar a análise automática, os cenários são transformados em Redes de Petri (Lugar/Transição) equivalentes. Os cenários e suas Redes de Petri resultantes podem ser analisados automaticamente para avaliar algumas propriedades relacionadas à desambiguidade, completeza, consistência e corretude. Os defeitos identificados podem ser rastreados até os cenários, permitindo a sua revisão. Nós também discutimos como desambiguidade, completeza, consistência e corretude das SRSs baseadas em cenários podem ser decompostas em propriedades relacionadas, e definimos heurísticas para encontrar indicadores de defeitos que prejudicam estas propriedades. Avaliamos nosso trabalho, aplicando a nossa abordagem de análise em quatro estudos de caso. Essa avaliação compara os resultados obtidos pela nossa abordagem automatizada contra os resultados obtidos por um processo de inspeção e com trabalhos relacionados.

\section{Palavras-chave}

Requisitos de software; cenários; casos de uso; análise de requisitos; verificação de requisitos; Petri-Net; Processamento de Linguagem Natural. 


\section{Contents}

1 Introduction $\quad 21$

1.1. Motivation 23

1.2. Problem 24

1.3. Objective 26

1.4. Thesis 26

1.5. Proposed Solution 26

1.5.1. Approach Overview 27

1.5.2. Expected Contribution 28

1.5.3. Evaluation 29

1.6. Outline 30

2 Theoretical Background 31

2.1. Requirements Engineering 31

2.1.1. Requirements 32

2.1.2. Requirements Specification 33

2.1.3. Scenario-Based Requirements Specification 34

2.1.3.1. Scenarios 34

2.1.3.2. Representing Scenarios 35

2.1.3.3. Use Case Representation 36

2.1.3.4. Scenario Representation 37

2.1.4. Natural Language-based Scenario Representations

$\begin{array}{ll}\text { Compared } & 38\end{array}$

2.2. Quality in Software Requirements Specification 39

2.2.1. Non-functional Requirements (NFR) 40

2.2.1.1. NFR Framework 40

2.2.2. Quality Assurance for Software Requirements 41

2.2.2.1. Software Requirements Quality Characteristics 42

2.2.2.2. Verification \& Validation 43

2.2.2.3. Quality of Scenarios 44 
2.3. Concurrency 45

2.3.1. Synchronization 46

2.3.2. Non-determinism 46

2.3.3. Synchronization Constraints $\quad 47$

2.3.4. Desired Properties of Concurrent Systems 47

2.3.4.1. Deadlock-free $\quad 47$

2.3.4.2. Boundedness $\quad 47$

2.3.5. Petri-Net 48

2.3.5.1. Petri-Net Definitions 48

2.3.5.2. Modeling with Petri-Nets 50

2.3.5.3. Analysis of Petri-Nets 51

2.4. Considerations about Scenarios and Concurrency 54

2.5. Related Work 54

2.5.1. Analysis of Software Requirements Specification 54

2.5.2. Overview of the State of the Art 55

2.5.2.1. Static Analysis of Software Requirements Specification 55

2.5.2.1.1. Static Analysis of Requirement Statements 56

2.5.2.1.2. Static Analysis of Scenarios $\quad 59$

2.5.2.2. Dynamic Analysis of Software Requirements Specification 62

2.5.3. Analysis Approaches Compared 65

2.5.4. Research Gaps 69

3 A Quality Model for Scenarios $\quad 71$

3.1. Quality in Scenario-based SRS 71

3.2. Modeling Correctness as Non-functional Requirements 72

3.2.1. Defining the Main NFRs 73

3.2.1.1. Unambiguity 74

3.2.1.2. Completeness 76

3.2.1.3. Consistency 80

3.2.1.4. Correctness 81

3.2.2. Modeling the SIG 81

3.3. Final Considerations 83

4 Scenario Analysis Approach $\quad 84$ 
4.1. Writing Restricted-form of Natural Language Scenarios

4.1.1. Scenario

4.1.1.1. Title

87

4.1.1.2. Goal

87

4.1.1.3. Context 88

4.1.1.4. Resources 88

4.1.1.5. Actors 88

4.1.1.6. Episodes 88

4.1.1.7. Exception 90

4.1.1.8. Constraint 90

4.1.2. Restricted-form of Natural Language 91

4.1.3. Scenario Relationships-based Modularity 94

4.1.3.1. Sequential Relationships 95

4.1.3.2. Non-sequential Relationships 96

4.1.3.3. Heuristics to Find Non-explicit and Non-sequential Relationships

4.1.4. Running Example 100

4.2. Pre-processing Scenarios 104

4.3. Deriving Petri-Nets 105

4.3.1. Transforming Scenarios into Petri-Nets 105

4.3.2. Integrating Petri-Nets 110

4.3.3. Petri-Net Example 112

4.3.4. Preservation of Properties 117

4.4. Analyzing Scenarios 118

4.4.1. Unambiguity Analysis $\quad 119$

4.4.2. Completeness Analysis 121

4.4.2.1. Lexical Analysis $\quad 122$

4.4.2.2. Syntactical Analysis 123

4.4.3. Consistency Analysis $\quad 125$

4.4.3.1. Managing the State Explosion 125

4.4.4. Correctness Analysis 130

4.5. Generating Feedback 130

4.5.1. Traceability between Petri-Net and Scenario 132 
4.6. Recommending Fixes for Defects 132

4.7. Final Considerations 135

$\begin{array}{ll}\text { 4.7.1. Complexity Analysis } & 136\end{array}$

5 C\&L (Cenários \& Léxicos) 138

5.1. C\&L 138

5.2. Extending C\&L - Lua 139

5.2.1. Tools 140

5.2.2. Modules 141

5.3. Implementation Details 142

5.3.1. Syntax Parser Module 142

5.3.1.1. Construct Scenarios 143

5.3.1.2. Identify Root Scenario 144

5.3.1.3. Construct Integration Scenario 145

§ 5.3.1.4. Operationalize Scenarios 145

5.3.2. Pre-processing Module 146

5.3.2.1. Construct Scenarios 146

5.3.2.2. Operationalize Scenarios 146

5.3.3. Petri-Net Generator Module 147

5.3.3.1. Construct Scenarios 148

5.3.3.2. Identify Root Scenario 149

5.3.3.3. Construct Integration Scenario 149

5.3.3.4. Operationalize Scenarios 149

5.3.4. Analysis Module 149

5.3.4.1. Construct Scenarios 149

5.3.4.2. Identify Root Scenario 150

5.3.4.3. Construct Integration Scenario 151

5.3.4.4. Operationalize Scenarios 151

5.3.4.4.1. String Finding 151

5.3.4.4.2. Regular Expression 152

5.3.4.4.3. Levenshtein's distance (Levenshtein, 1966) 152

5.3.4.4.4. Phrase-structure Parsing 152

5.3.4.4.5. Syntactic Similarity Heuristic 156 
5.3.4.4.6. Reachability Analysis:

159

5.3.5. Feedback Generator Module 160

5.3.5.1. Construct Scenarios 161

5.3.5.2. Operationalize Scenarios 161

5.4. Usage 162

5.4.1. C\&L Main Menu 163

5.4.2. C\&L Scenario and Lexicon Functionalities 164

5.4.3. C\&L Analysis Functionality 165

5.4.4. C\&L Petri-Net Visualizer Functionality 167

5.5. Final Considerations 168

6 Case Studies 169

6.1. Introduction 169

6.1.1. Hypothesis 169

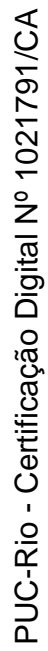

6.1.2. Variables 170

6.1.3. Evaluation Metrics 170

6.1.4. Case Study Selection 171

6.1.5. Subjects 173

6.2. Referential Baseline Solution 174

6.2.1. Online Broker System (Somé, 2010) 174

6.2.2. ATM system (Cox et al., 2004) 175

6.2.3. DLibra and Mobile News 176

6.2.4. Summary of Baselines 177

6.3. Evaluation 178

6.3.1. Time Analysis 178

6.3.2. Analysis Results 179

6.3.2.1. Results of Unambiguity Analysis 180

6.3.2.2. Results of Completeness Analysis 181

6.3.2.3. Results of Consistency Analysis 182

6.3.2.4. Results of Correctness Analysis 184

6.4. Interpretation 184

6.4.1. Accuracy of the Petri-Net Generator 185

6.4.2. Considerations about Scalability 186 
$\begin{array}{ll}\text { 6.5. Threats to Validity } & 187\end{array}$

$\begin{array}{lr}\text { 6.6. Conclusion } & 187\end{array}$

7 Conclusion 189

$\begin{array}{ll}\text { 7.1. Comparison with Related Work } & 191\end{array}$

7.2. Contribution 193

7.3. Limitation 194

$\begin{array}{ll}\text { 7.4. Future Work } & 195\end{array}$

$\begin{array}{ll}\text { References } & 196\end{array}$

Appendix A1 Referential Specification Used as Baseline 202

A1.1 The Online Broker System 203

A1.2 The ATM System 206

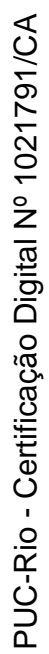

A1.3 DLibra CRM 210

$\begin{array}{ll}\text { A1.4 Mobile News } & 217\end{array}$

Appendix A2 Quality Models of Related Work 224

A.2.1. Static Analysis of Software Requirements Specification 224

A.2.1.1. Static Analysis of Requirement Statements 224

A.2.1.2. Static Analysis of Scenarios 226

A.2.2. Dynamic Analysis of Software Requirements Specification $\quad 230$ 


\section{List of Figures}

Figure 1 - Overview of the Scenarios Analysis Approach. 28

Figure 2 - Requirement Engineering (Leite, 2007) 32

Figure 3 - SIG of Correctness. 41

Figure 4 - Producer and Consumer Problem Using Petri-Nets 45

Figure 5 - Reader and Writer Problem Using Petri-Nets 46

Figure 6 - Petri-Net metamodel (Sarmiento et al., 2015) 48

Figure 7 - Marked Petri-Net $\quad 49$

Figure 8 - (a) Transitions before Firing, (b) Transitions after firing 50

Figure 9 - Sequential structure $\quad 50$

Figure 10 - Non-deterministic structure $\quad 51$

Figure 11 - Concurrency structure $\quad 51$

Figure 12 - Synchronization structure $\quad 51$

Figure 13 - (a) Transitions before Firing, (b) Transitions after firing 52

Figure 14 - A Reachable Petri-Net (generated using PIPE2, 2015) 53

Figure 15 - Initial SIG of SRS Correctness. 73

Figure 16 - SIG of SRS Correctness. 83

Figure 17- SADT of the Scenarios Analysis Approach. 85

$\begin{array}{ll}\text { Figure } 18 \text { - Scenario Conceptual Model. } & 87\end{array}$

Figure 19 - Example of scenario (Submit Order) in the Online Broker

$\begin{array}{lr}\text { System. } & 91\end{array}$

Figure 20 - Making Explicit Non-sequential Relationships (Heuristic 1). 100

Figure 21 - "Submit Order" use case in the Online Broker System (Somé, 2010).

Figure 22 - Description of scenario "Submit Order" in the Online Broker $\begin{array}{ll}\text { System. } & 102\end{array}$

Figure 23 - Scenarios of the "Online Broker System". 103

Figure 24 - Transforming Simple Episode 109 
Figure 25 - Mapping scenario constructs into Petri-Net elements.

Figure 26 - Transform Scenario into Petri-Net (Method 1).

Figure 27 - Integrate Petri-Nets (Method 2).

Figure 28 - Register Customer (a), Submit Order (b) and Process Bids

(c) Petri-Nets.

Figure 29 - Integrated Petri-Net of "Submit Order".

Figure 30 - Substitution input place (a) and concurrent fusion place (b). 118

Figure 31 - Unambiguity Analysis (Method 3).

Figure 32 - Lexical analysis of simple episode (a) and exception (b) elements.

Figure 33 - Parse tree for verb-object (a), subject-verb-object (b) and subject-verb-object-indirect-object (c) sentences.

Figure 34 - Completeness Analysis (Method 4).

Figure 35 - Consistency Analysis (Methoid 5).

Figure 36 - Integrating "Suppliers" Petri-Nets into the Petri-Net of "Submit Order".

Figure 37 - Reachability graph (a) and Reachability analysis results (b) of "Submit Order" scenario.

Figure 38 - C\&L - Lua Architecture (Sarmiento et al., 2014).

Figure 39 - High Level Architecture of Extended C\&L

Figure 40 - Scenario to Identify the Scenario Elements

Figure 41 -Scenario to Verify the Main Components of Scenario Context

Figure 42 -Scenario to Verify the Main Components of Scenario Resource

Figure 43 - Scenario to Verify the Main Components of Scenario Episodes

Figure 44 - Scenario to Verify the Main Components of Scenario Exceptions

Figure 45 - Relationships among scenarios of Syntax Parser module

Figure 46 - Integration Scenario of Syntax Parser Module

Figure 47 - Scenario to Clean Scenario of Irrelevant Information

Figure 48- Scenario to transform a Scenario into a Petri-Net 
$\begin{array}{ll}\text { Figure } 49 \text { - Scenario to integrate a set of related Petri-Nets } & 148\end{array}$

Figure 50 - Scenario to Analyze Unambiguity 150

Figure 51 - Scenario to Analyze Completeness 150

Figure 52 - Scenario to Analyze Consistency 150

Figure 53 - Scenario to Analyze Scenario 151

Figure 54 - String Finding Operationalization 152

Figure 55 - NLP Tags (Compendium-js, 2015) 153

Figure 56 - Get sentence components method (Subject, Action-Verb and Objects). 156

Figure 57 - Syntactic Similarity Implementation. 158

Figure 58 - Reachability Analysis on PIPE2. 160

Figure 59 - Scenario to Generate Feedback 161

Figure 60 - Initial page of the C\&L. 163

Figure 61 - Integration scenario to use the C\&L. 164

$\underset{Ð}{\circlearrowleft} \quad$ Figure 62 - Add lexicon symbol and add scenario forms. 164

Figure 63 - Visualize Project Form. 165

Figure 64 - Visualize Scenario Form. 166

Figure 65 - Project Analysis Feedback Interface (1). 166

Figure 66 - Project Analysis Feedback Interface (2). 167

Figure 67 - Petri-Net Visualization Interface. 168

Figure 68 - Relation between case study length and average processing $\begin{array}{ll}\text { time. } & 179\end{array}$

Figure 69 - Consistency Analysis Using Petri-Nets in "Broker System”. 183

Figure 70 - Consistency Analysis Using Petri-Nets in "Mobile News". 184 


\section{List of Tables}

Table 1 - Use Case Template (Cockburn, 2001) 37

Table 2- Scenario template (Leite et al., 2000) 38

Table 3 - Scenario and Use Case Comparison 39

Table 4 - Comparing Requirement Statements Static Analysis Techniques $\quad 66$

Table 5 - Comparing Scenarios Static Analysis Techniques 67

Table 6 - Comparing Requirement Statements Dynamic Analysis $\begin{array}{ll}\text { Techniques } & 68\end{array}$

Table 7 - Properties Related to Unambiguity. 75

Table 8 - Intra-scenario Properties Related to Completeness (Continued on Table 9).

Table 9 - Intra-scenario Properties Related to Completeness. 78

Table 10 - Inter-scenario Properties Related to Completeness. 79

Table 11 - Feasibility Property Related to Completeness. 80

Table 12 -Properties Related to Consistency. 81

Table 13 - Scenario Grammar 92

Table 14 - Proximity Index between Scenarios of the Online Broker $\begin{array}{ll}\text { System } & 104\end{array}$

Table 15 - Transforming Scenario Triggering 107

$\begin{array}{ll}\text { Table } 16 \text { - Transforming Episode } & 107\end{array}$

Table 17 - Transforming Concurrency Construct 108

Table 18 - Transforming Exception 108

Table 19 - Transforming Scenario Completion 108

Table 20 - Scenario Defects Classification 131

Table 21 - Recommendations for Analyzing Unambiguity Properties. 133

Table 22 - Recommendations for Analyzing Completeness (Intra-

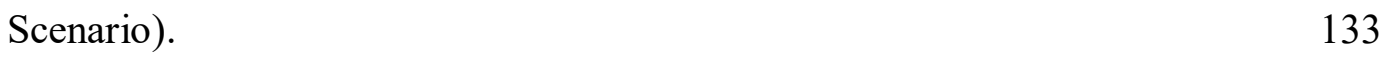

Table 23 - Recommendations for Analyzing Completeness (Intra-

$\begin{array}{ll}\text { Scenario). } & 134\end{array}$

Table 24 - Recommendations for Analyzing Completeness (InterScenario). 
Table 25 - Recommendations for Analyzing Consistency Properties.

Table 26 - Symbol definition in lexicon language.

Table 27 - tagging Examples using NLP Tools

Table 28 - Rules to Extract Action-Verbs and Nouns

Table 29 - Intra-scenario Properties Related to Completeness.

Table 30 - Characteristics of the Case Studies

Table 31 - Summary of the Baseline for the Case Studies

Table 32 - Analysis of Unambiguity using the C\&L - Lua.

Table 33 - Analysis of Completeness using the C\&L - Lua.

Table 34 - Analysis of Consistency using the C\&L - Lua.

Table 35 - Analysis of Correctness using the C\&L - Lua.

Table 36 - Characteristics of the Admission System Case Study 186

Table 37 - Comparing SRS Analysis Techniques 193

Table 38 - Quantitative Analysis of Online Broke System 203

Table 39 - Unambiguity Analysis of Online Broke System

Table 40 - Completeness Analysis of Online Broke System

Table 41 - Consistency Analysis of Online Broke System

Table 42 - Quantitative Analysis of ATM System 206

Table 43 - Unambiguity Analysis of ATM System 207

Table 44 - Completeness Analysis of ATM System 207

Table 45 - Consistency Analysis of ATM System 207

Table 46 - Quantitative Analysis of Online Broke System 210

Table 47 - Unambiguity Analysis of Dlibra System 210

Table 48 - Completeness Analysis of Dlibra System 211

Table 49 - Quantitative Analysis of Mobile News System 217

Table 50 - Unambiguity Analysis of Mobile News System 217

Table 51 - Completeness Analysis of Mobile News System 218

Table 52 - Quality Indicators of ARM (Wilson et al., 1997) 224

Table 53 - Expressiveness Quality Model of QuARS (Gnesi et al., 2005) 224

Table 54 - Ambiguity Indicators of SRRE (Tjong, 2008) 225

Table 55 - Requirements language criteria (IEEE, 2011; Femmer et al., 2014)

Table 56 - Potentially problematic constructs (from Berry et al., 2012) 225 
Table 57 - Quality User Story Framework (Lucassen et al., 2015)

Table 58 - Taxonomy of defects in use case models (Anda and Sjoberg, 2002)

Table 59 - Scenario Checklist (Leite et al., 2000; Leite et al., 2005)

Table 60 - The 7Cs Verification Heuristics (Phalp et al., 2007) 228

Table 61 - The Use Case Defects (Ciemniewska and Jurkiewicz, 2007) 229

Table 62 - Use Case Checklist of Text2Test (Sinha et al., 2010) 230

Table 63 - Common use case defects (Liu et al., 2014) 230

Table 64 - Consistency and Completeness in CMPN (Lee et al.,1998) 230

Table 65 - Faults Detected by Time Petri-Nets (Lee et al., 2001) 231

Table 66 - Use Case Defect Classification (Denger et al., 2005) 231

Table 67 - Properties of UC-LTSs (Sinnig et al., 2009) 231

Table 68 - Properties of Timed and Controlled Petri-Nets (Zhao and Duan, 2009) 231

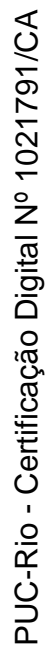

Table 69 - Properties of Reactive Petri-Nets (Somé, 2010) 


\section{List of Abbreviations}

\begin{tabular}{|c|c|}
\hline BNF & Backus Normal Form \\
\hline C\&L & Cenários e Léxico \\
\hline CSP & Communicating Sequential Processes \\
\hline DEO & Discrepancies, Errors, and Omissions \\
\hline IEEE & Institute of Electrical and Electronics Engineers \\
\hline ISO & International Standards Organization \\
\hline FR & Functional requirements \\
\hline GORE & Goal-Oriented Requirements Engineering Approaches \\
\hline LTS & Labeled Transition Systems \\
\hline LEL & Language Extended Lexicon \\
\hline LSC & Live Sequence Charts \\
\hline MSC & Message Sequence Charts \\
\hline MVC & Model-View-Controller \\
\hline NL & Natural Language \\
\hline NLP & Natural Language Processing \\
\hline NFR & Non-functional requirements \\
\hline POS & Part-of-Speech - POS \\
\hline PN & Petri-Nets \\
\hline PNML & Petri Net Markup Language \\
\hline $\mathrm{RE}$ & Requirements Engineering \\
\hline RNL & Restricted-form of natural language \\
\hline SADT & Structured Analysis and Design Technique \\
\hline SIG & Soft-goals Interdependency Graph \\
\hline SD & Software Development \\
\hline SRS & Software Requirements Specification \\
\hline UML & Unified Modeling Language \\
\hline UofD & Universe of Discourse \\
\hline
\end{tabular}




\section{1 \\ Introduction}

Many research studies have shown how the Requirements Engineering (RE) activities play an important role in the reliability, cost and safety of a software system; especially, the importance of early requirements analysis on the reduction of the development costs, confusion and complexity in the later activities of Software Development (SD). RE activities are important mainly for two reasons. First, they help software development stakeholders to better understand and communicate the software requirements. Second, their main output, i.e. the Software Requirements Specification (SRS) serves as the basis for later software development activities, e.g., design, coding and testing.

Therefore, it is highly desirable to produce a Software Requirements Specification with a good quality, i.e., a SRS that is more correct, consistent, complete, unambiguous, understandable and traceable (IEEE, 1998, Lee et al., 1998; Glinz, 2000; Lee et al., 2001; Cheung et al, 2006; Somé, 2010; Zhao and Duan, 2009).

Requirements Analysis includes activities related to verification and validation (Leite, 2007), such as finding defects in structural and behavioral properties of SRS documents and addressing problems related to these properties, which could reduce most of the risks in the later activities of software development. However, requirements analysis is still an extensive and iterative process, which is mostly performed manually, requiring a great effort and taking a lot of time.

Requirements described through rigorous or tabular specifications enable automated analysis by simulating a sequence of events that represents a narrow aspect of a system's required behavior; these detect several classes of faults by checking specification properties (Lamsweerde et al., 1998; Heitmeyer, 2007). However, these practices are expensive and not widely used in industrial practice.

For practical reasons, and in order to allow for an easy communication with stakeholders, informal or semiformal representations are widely used by user- 
oriented approaches. User-oriented approaches are dominant during Requirements Engineering activities in industry; and, one of the key elements in this perspective is the notion of scenarios. In this context, a SRS is represented as a collection of scenarios and described by specific flows of events and their guard conditions. The use of scenarios helps understanding a specific situation in an application, prioritizing their behavior (Leite et al., 2000). Some of the most prominent languages to write scenarios are restricted-form of use case descriptions (Cockburn, 2001), scenario descriptions (Leite et al., 2000), UML dynamic behavior (UML, 2015) diagrams and Message Sequence Charts (Andersson and Bergstrand, 1995).

The graphical notation based languages for writing scenarios are very attractive and user-friendly; however, they can be difficult to design, and domain experts cannot reasonably be asked to draw them (Gutiérrez, 2008). Although these languages provide an accessible visualization of models, they lack formal semantics to support the analysis of structural and behavioral properties of the modeled system.

According to Glinz, (2000), in the literature, there is no clear distinction between natural language-based scenarios and use case descriptions, both scenarios and use cases describe situations that could possibly happen between the users and a system. However, the scenario language proposed by Leite et al (2000) also helps on understanding specific situations in an application, prioritizing their behavior.

In this thesis, scenario and use case are considered synonymous because they are described by similar components. There are several different templates or syntax for writing scenarios, and some of the most common components used to detail scenarios are: Title/Name, Goal, Pre-condition, Post-condition, Actors, Episodes/Main Flow and Exceptions/Alternative Flows.

Unfortunately, natural language-based scenarios exhibit some shortcomings: (1) informally specified scenarios are usually hard to analyze, because natural language is by definition ambiguous; (2) modularity is poorly supported, because the relationships among scenarios are rarely represented explicitly; and (3) currently, there are no systematic approaches to identify and make explicit potential concurrency issues (e.g. deadlock, non-determinism) in initial requirements descriptions. 
According to Lee et al. (1998), although such subsets of scenarios might seemingly be independent, they are rarely truly independent in practice. A set of scenarios can be considered as a set of concurrently executing threads. Thus, from the concurrency perspective, scenarios are rarely truly independent in practice; they may interact or compete with each other by communication channels or shared resources, what can lead to erroneous situations such as deadlocks.

\section{1.}

\section{Motivation}

Because of inherent ambiguity of natural language (NL), defects are inevitably introduced into scenario-based SRS. Thus, assessing the quality of a SRS document is not a simple process, mainly, because:

- Finding defects in scenarios is an important activity mostly performed manually, which is expensive, time-consuming and error-prone.

- Multiple users with different viewpoints and conflicting needs about the system are involved at RE activities.

- Relationships among scenarios are rarely represented explicitly (Lee et al., 1998, Leite et al., 2000). Scenarios are related to other scenarios by sequential (precedence order) and non-sequential relationships (indistinct sequential order, concurrency or parallelism).

- Heuristics for finding non-explicit relationships among scenarios are rarely proposed (Leite et al., 2005).

- Finding defects from the relationships among different scenarios is a complex activity. It is necessary to execute (or simulate the behavior) a set of scenarios for detecting defects from the relationships among them (Denger et al., 2005).

- Most of the defects found by analysis techniques are in fact simple linguistic defects in single scenarios (Adapted from Gnesi et al., 2005).

Ambiguity may lead to incomplete, inconsistent and incorrect scenariobased SRS documents. Since a scenario-based SRS describes requirements statements using scenarios and their relationships: Ambiguity occurs when two or more users have different interpretations of the same requirement statement stated in a single scenario. Incompleteness in a single scenario or involving multiple 
scenarios occurs because the world is complex; as such, users or clients are not able to identify and develop all relevant requirements within scenarios. Inconsistency occurs when two or more users have conflicting or overriding requirements, thus, scenarios can overlap other scenarios. Incorrect scenarios occur when the acquired requirements do not accurately reflect the facts, or erroneous predicts about future states.

Some examples of defects that hurt Unambiguity, Completeness, and Consistency quality properties in scenario-based SRS include:

- Unambiguity: Different interpretations of the same requirement;

○ Title contains subjective words or phrases (e.g., similar, better);

○ Episode contains weak words or phrases (e.g., can, might);

- Completeness: Fully developed requirement statements;

- Actor does not participate in any episode of the main flow;

- Conditional episode is not conform to the syntax rules;

- Related Scenario does not exist in the set of Scenarios;

- Consistency: Free of conflicting or overriding requirement statements;

- Pre-condition coincidence: non-determinism (warning);

- Bi-directional reference among related scenarios (circular inclusion);

- Never enabled sequence of episodes (or steps);

Usually, these defects are fixed in software design activities; however, ambiguity, incompleteness, inconsistency and incorrectness in scenarios must be resolved in early activities of software development (i.e., RE activities). It increases the software reliability and improves the productivity of software development (Lee et al., 1998). The importance of SRS quality has been recognized by several studies (Boehm and Basili, 2001; Bernstein and Yuhas, 2005).

\section{2.}

\section{Problem}

Scenario specifications are usually informal or semi-formal, and in these cases, they are not the best choice for further automated analysis (including graphical notation based models) because they lack of formal semantics to support 
it. Thus, there is a lack of formal semantics to support the early analysis of structural and behavioral properties of systems described as scenarios.

Several research studies have shown the importance to formalize scenarios through restricted-form of use case descriptions (Somé, 2010), Message Sequence Charts - MSC (Andersson, and Bergstrand, 1995; Damas et al., 2006), Live Sequence Charts - LSC (Damm and Harel, 2001) or BNF-like gramar (Hsia et al., 1994); other researchers have used concepts from Petri-Nets (Lee et al., 1998; Lee et al., 2001; Cheung et al., 2006; Zhao and Duan, 2009; Somé, 2010), Statecharts (Glinz, 2000; Denger et al., 2005), Labeled Transition Systems - LTS (Sinnig et al., 2007) or Communicating Sequential Processes - CSP (Cabral and Sampaio, 2006).

These literature circles argue for the need for a precise representation for scenarios in order to be useful in automated analysis, model derivation or test generation. In these approaches, scenarios are described by a variety of scenario notations, in some cases with rigorous semantics; scenarios are used to document system requirements, then, scenarios are translated into Petri-Nets - PN (Murata, 1989), LTS (Keller, 1976) or CSP (Roscoe, 1998); which are used as the mechanism to enable rigorous analysis. The resulting formal models can be further processed and analyzed using available tools to verify structural and behavioral properties, ensuring mainly the consistency and correctness.

The translation-based approaches are difficult to apply because it requires a strong knowledge and experience on formal modeling for translating initial scenarios into formal models. Other drawbacks are:

- There is no consensus on how to represent scenarios; some languages depend on formal definition of pre-conditions and post-conditions within single scenarios (Lee et al., 1998; Sinnig et al., 2009);

- Most of the existing approaches do not provide formal definition of translation rules between scenario elements and formal model elements, which can make the automation more difficult;

- Most of the existing approaches do not provide procedures for integrating a set of related scenarios into a whole representation, and detect defects from these relationships. Scenarios interact by sequential and nonsequential relationships; 
- In most of the existing approaches, the use of the analysis feedback of equivalent formal models to improve the scenario descriptions is difficult, since they do not provide ways of tracing to defects in the original scenario.

\section{3.}

Objective

Motivated by the importance of improving the quality of Software Requirements Specification documents based on scenario representations, we are propose a new approach for scenarios analysis that is based on Petri-Nets and Natural Language Processing (NLP) techniques, which exploits inter-scenario relationships to overcome major unsolved problems and improve the state of the art.

1.4.

Thesis

"NATURAL LANGUAGE-BASED SCENARIOS CAN BE ANALYZED THROUGH PETRI-NETS AND NLP” BY AN APPROACH THAT:

- Show defects that hurt unambiguity in single scenarios at RE;

- Show defects that hurt completeness in single scenarios at RE;

- Show defects that hurt completeness from relationships among scenarios at RE;

- Show defects that hurt consistency and correctness in Petri-Nets derived from scenarios and their relationships at RE;

- Support modularity by proposing heuristics for finding explicit and nonexplicit relationships among scenarios;

- Support traceability by indicating defects in Petri-Nets and showing the source of the defects in scenarios;

- Can be implemented through a software tool.

\section{5.}

Proposed Solution

As scenarios are useful in other development activities, these scenarios must be correct and valid. Therefore, effectiveness of scenarios analysis could be significantly improved by an approach, which could discover defects that are hidden in scenarios and their relationships in an automatic way. The higher goal 
of this thesis is to develop a "Petri-Net and NLP based Approach as an Effective Way to Analyze the Acquired Scenarios", which evaluates structural and behavioral properties related to Unambiguity, Completeness, Consistency and Correctness. The following goals refine the stated goal:

- Define a restricted-form of natural language (RNL) to write scenarios;

- Develop heuristics for finding non-explicit relationships among scenarios;

- Develop a systematic procedure that transforms scenarios stated in a RNL to Petri-Nets;

- Improve the existing NLP Parsing strategies to correctly identify the Subject, Objects and Action-Verb in textual scenario sentences.

- Employ the non-functional requirements (NFR) approach to:

○ Model the relationships between unambiguity, completeness, consistency and correctness qualities of scenarios;

- Identify the properties related to unambiguity, completeness, consistency and correctness;

- Develop heuristics for searching defect indicators that hurt properties related to unambiguity, completeness, consistency and correctness qualities.

\subsection{1.}

\section{Approach Overview}

Our scenarios analysis approach checks the acquired scenarios by detecting wrong information, missing information and erroneous situations that can be hidden within scenarios and their relationships. In this regard, we instantiate a Quality Model for Scenarios (defined in this thesis), and consider the results achieved by NLP and Petri-Net based related work.

The related work in using the potential of Petri-Nets for scenario formalization indicates that Petri-Nets are an effective mechanism for scenariobased SRS analysis. The motivation behind translating scenarios into Petri-Nets can be attributed to three reasons: First, the reachability analysis can reveal the incorrect behavior of a set of scenarios (mapped into Petri-Nets); Second, the availability of Petri-Net tools, such as PIPE2 (2015); and Third, the portability of Petri-Net models (interchangeable format between tools - Petri Net Markup Language - PNML). 
So, in our approach: First, requirements engineers start to describe the different functionalities, services or situations of the system as scenarios using a RNL. Second, irrelevant information within scenario elements are removed. Third, in order to perform an automated analysis of scenarios, an initial system design is derived by translating these scenarios into Place/Transition Petri-Nets, and synthesizing them into a consistent whole Petri-Net. Fourth, scenarios and their resulting Petri-Nets are automatically analyzed to evaluate some properties related to unambiguity, completeness, consistency and correctness. Fifth, the analysis outcome is formatted and returned to the requirements engineers. Sixth, if defects are found, the analysis feedback is used to improve the scenario descriptions, since the identified defects and their causes can be traced to the scenarios. Figure 1 depicts an overview of our approach. The different phases of our approach were implemented in the C\&L (2010) prototype tool.

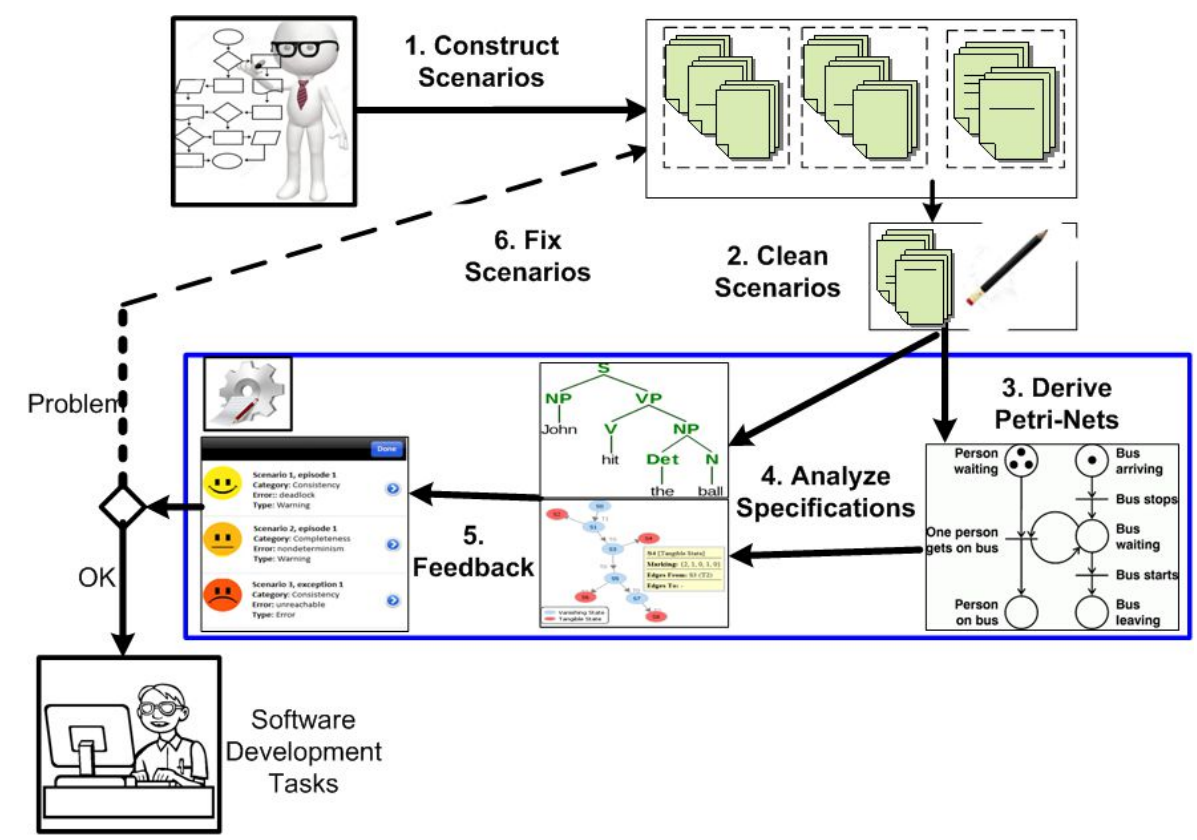

Figure 1 - Overview of the Scenarios Analysis Approach.

\subsection{2.}

\section{Expected Contribution}

The main contribution of this thesis is an automated analysis approach of structural and behavioral properties in scenario specifications. The analysis is able to detect defects that provide evidence that the properties related to Unambiguity, Completeness, Consistency and Correctness were violated. 
This approach benefits from both the precision of graphical Petri-Nets and the usability of textual scenarios; and it also allows an easier integration to available Petri-Net tools like PIPE2 (2015).

The objective of this thesis is to contribute with the following results:

- The definition of a Restricted-form of Natural Language-based Scenario Model, whose elements may be written using a semi-structured linguistic grammar. This scenario language was initially proposed by Leite et al (2000).

- The definition of Heuristics for Finding Non-explicit Relationships among scenarios: Scenarios are related to other scenarios by sequential (precedence order) and non-sequential relationships. Frequently, nonsequential relationships are non-explicit.

- The definition of a reusable Quality Model for Scenarios, which organizes the properties related to Unambiguity, Completeness, Consistency and Correctness. These properties were based on previous work (Leite et al., 2000) and related work.

- A procedure to Translate Scenarios stated in a restricted-form of natural language into Petri-Nets, preserving the consistency between these equivalent representations.

- A systematic procedure to synthesize a system design from the resulting Petri-Nets, preserving the original properties of synthesized Petri-Nets.

- Manage the State Explosion Issue (Lee et al., 1998): State explosion issue is a serious problem when applying Petri-Net analysis to large systems. A contribution of this thesis is a MULTI-STEP BOTTOM-UP analysis approach to manage this problem.

- The development of the C\&L tool (Cenários \& Léxicos): An experimental tool that automatically detects potential defects in scenarios. For every potential defects detected, C\&L shows it to the user in a understandable way.

\subsection{3.}

\section{Evaluation}

Five case studies with different degree of complexity were carried out to evaluate the accuracy and the scalability of the proposed analysis approach. We 
evaluated the accuracy of results produced by the developed tool (C\&L-Lua) with respect to reference solutions elaborated by expert Requirements Engineers of different universities. These set of scenarios have a near-typical profile, i.e., they contain typical defects in industrial projects (UCDB, 2015).

\section{6.}

\section{Outline}

This thesis is organized as follows:

Chapter 2: Presents a general introduction of Requirements Engineering, Concurrency and Petri-Nets. It also presents the state of the art in requirements analysis, compare the different existing approaches, and identify the most important research gaps.

Chapter 3: Presents a reusable Quality Model for Scenarios by modeling the relationships between unambiguity, completeness, consistency and correctness qualities.

Chapter 4: Presents the proposed approach for scenarios analysis. It includes the proposed scenario language, which is used to write scenarios enabling further transformations into executable representations; the procedure for translating scenarios into Petri-Nets; the strategy for evaluating structural and behavioral properties of scenarios; and the strategy for managing the state explosion issue of Petri-Nets.

Chapter 5: Presents the C\&L tool architecture and its implementation. An experimental tool that automatically detects potential defects in scenarios

Chapter 6: Shows the evaluation of the developed approach by finding defects in scenario specifications.

Chapter 7: Discusses the differences between our work and those related work and summarize our improvements on the state-of-the-art. Presents the conclusions, limitations, and some suggestions for future work. 


\section{2 \\ Theoretical Background}

This chapter begins with a general overview of Requirements Engineering and techniques to document and analyze scenarios (Section 2.1). Next, Section 2.2 investigates the topic of requirements quality. Concurrency and Petri-Nets are introduced in a comprehensive way in Section 2.3. Section 2.4 discusses research (or tools) related to the analysis of Natural Language based SRSs, compares techniques to analyze static and dynamic aspects related to quality attributes of requirements in a comprehensive way, and highlight research gaps. Finally, Section 2.5 concludes by discussing the relationships between scenarios and concurrent systems.

\section{1.}

\section{Requirements Engineering}

The development of software systems with acceptable quality and lower cost is a constant concern for the software development industry as well as it customers. An erroneous or incomplete understanding of the problem that software aims to solve may lead to software systems correctly implemented, but missing the customer needs. One of the main success factors at the activities involved in the $\boldsymbol{S D}$ process, is the correct understanding of the problem domain, i.e., a more clear and precise $\boldsymbol{S R S}$; but this is not always possible because of multiple stakeholders involved in the software development process, with different needs, assumptions and points of view of the domain. Thus, Requirements engineering is closely related to the good quality of software systems; thus, it is a key factor for successful software development companies.

Requirements Engineering (RE) is one of the most crucial and complex activities in software development and bridges the gap between customers needs and software engineering. According to Pohl (1994), requirements engineering may be understood as a process with a set of activities; where the desired output of this process is a document (SRS - Software Requirements Specification) expressed using a formal language on which most of stakeholders agree. This 
process inputs are user's points of view (usually ambiguous) of the system to be built; these inputs are obtained using an informal language (or making use of graphics), usually natural language.

Similarly, Leite (2007) subdivided the Requirements engineering into elicitation, modeling, analysis, and management activities (Figure 2). Elicitation is the first step and responsible to identify most of relevant key stakeholders and discover what they need. Modeling is the process of building abstract descriptions of the requirements that are amenable to interpretation. Analysis corresponds to the generation of a SRS with acceptable quality and it is subdivided into verification and validation. Requirements management is transversal to RE process, and it consists of version control, change control, and traceability of the requirements (Leite, 2007). The input of this process is the Universe of Discourse (UofD), i.e., it includes all the sources of information and all the people related to the software.

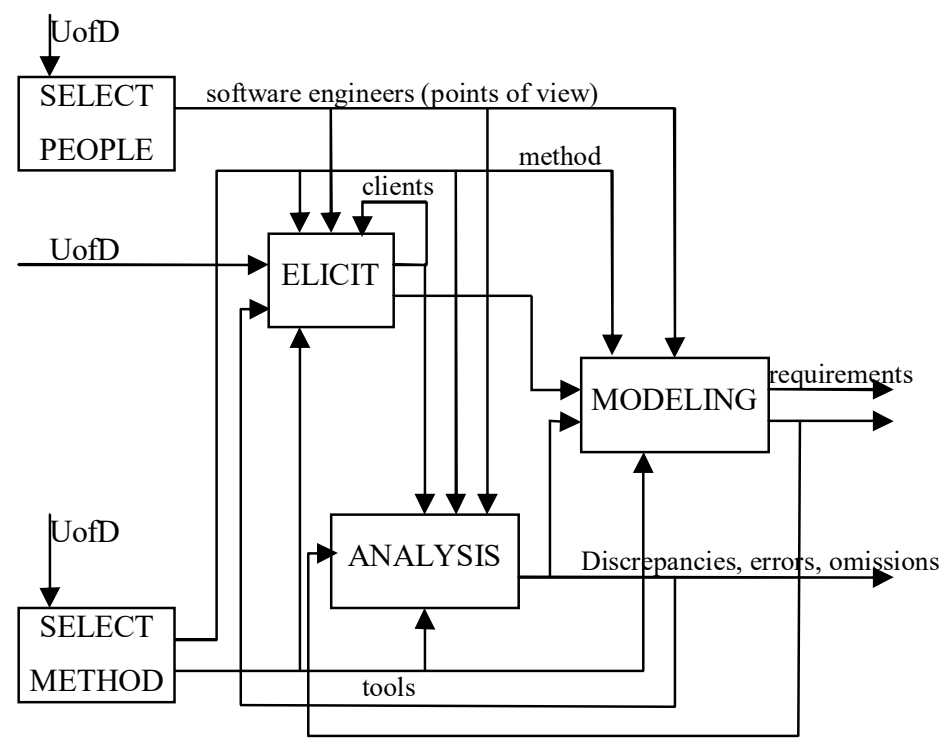

Figure 2 - Requirement Engineering (Leite, 2007)

\subsection{1.}

\section{Requirements}

Poorly defined requirements are the major cause for software projects to fail. Requirements in software engineering are descriptions of actions, behavior and constraints of a system in order to meet stakeholders' needs. According to Sommerville (2010), requirement is the specification of what is to be implemented, and he classified requirements into functional and non-functional 
requirements and distinguished between two different levels of abstraction: user requirements and system requirements.

- Functional requirements (FR) define the behavior of the system and what it should do.

- Non-functional requirements (NFR) also known as quality attributes (QA) or soft-goals (van Lamsweerde, 2001), describe the system attributes and define the constraints of a system.

- User requirements define the software functionality from a user perspective. They define what the software has to do to accomplish the user's goals.

- System requirements are more detailed description of software functions, services and operational constraints.

\subsection{2.}

\section{Requirements Specification}

Once the requirements are gathered, they need to be described. Requirements are expressed in a software requirements document, which is also called a SRS. The document includes requirements definitions, requirements specifications or system models. Usually, requirements definitions and requirements specifications are presented separately. However, in some cases these two are incorporated into a single description.

A requirements definition is a high-level abstract statement of the services a system must provide and the constraints it must meet. It is expressed in a natural language and without any reference to a solution, and targeted mainly at clients and project managers. The definition is based on information supplied by clients or users.

A requirements specification (also called a functional specification) is a structured description describing the services the system must meet in a more detailed manner. It may contain references to technologies or solutions. The description may serve as a contract between software developers and customers. The requirements specification expands the requirements definition and is targeted mainly at project managers and software developers.

Sommerville (2010) called requirements definition, a user requirements specification; and requirements specification, a system requirements specification. 
To document software requirements there are various techniques and languages, which may be classified as being informal, formal or semi-formal.

- Informal techniques use unrestricted natural language to document requirements. The advantage of natural language is that it is universal and flexible, but unfortunately is ambiguous.

- Formal languages are based on rigorous mathematical or logical reasoning for which the syntax, semantics and rules are explicitly defined (e.g. Temporal Logic, First Order Logic, SCR, Z, B, Petri-Nets). These methods are accurate and eliminate ambiguity, but they are hard to understand without a specific training and require a formal background.

- Semi-formal techniques include diagrams and tabular techniques that present information in a structured form (e.g. UML diagrams, Message Sequence Charts diagrams, ER models). They try to close the gap between two previous techniques.

\subsection{3.}

\section{Scenario-Based Requirements Specification}

While requirements are statements describing the expected system services, scenario-based representations have attracted considerable attention in RE. A scenario describes a situation that could possibly happen in a system.

In this context, a SRS document could be described as a collection of scenarios and each scenario is described by specific flows of events and their guard conditions. The use of scenarios helps understanding a specific situation in an application, prioritizing their behavior (Leite et al., 2000).

The main purpose of scenarios is to stimulate thinking about possible events, opportunities and risks in a system. They are often applied to model and communicate requirements among stakeholders due to their comprehensibility (Glinz, 2000; Leite et al., 2000).

\subsubsection{1. Scenarios}

According to Leite et al. (2000), the word scenario has a particular meaning in the software engineering community. It is "a description technique that is both process-focused and user centric". It is widely used in requirements engineering 
because it helps engineers to better understand the software requirements and its interface with the environment.

According to Glinz (2000), in software engineering, scenarios are described as "an ordered set of interactions between partners, usually between a system and a set of actors external to the system". Other researchers have similar definitions. For instance, Leite at al. (2000) defines a scenario as a partial description of the application behavior that occurs at a given moment in a specific geographical context - a situation. Van Lamsweerde and Willemet (1998) framed the term to "temporal sequence of interactions among different agents in the restricted context of achieving some implicit purpose".

In literature there is no clear distinction between scenarios and use cases. While some authors consider that each scenario corresponds to one use case (Glinz, 2000), others define a scenario as sequences of use case steps that represent different paths through a use case (Cockburn, 2001). According to Glinz (2000), a scenario may comprise a concrete sequence of interaction steps (instance scenario) or a set of possible interaction steps (type scenario).

\subsubsection{2.}

\section{Representing Scenarios}

There are a wide variety of scenarios representations in the literature, each one with quite different purposes. Therefore, scenarios can take many forms and provide various types of information on different levels of abstraction and formalism. Some of the most prominent languages to write scenarios are semistructured-form of use case descriptions (Cockburn, 2001), restricted-form of scenario descriptions (Leite at al., 2000), UML sequence diagrams (UML, 2015), UML activity diagrams (UML, 2015), MSC (Andersson and Bergstrand, 1995), LSC (Damm and Harel, 2001), StateCharts (Harel, 1987) or Petri-Nets (Murata, 1989).

There are several styles in which scenarios are written. In system development, Alexander and Maiden (2005) defined six common types of scenarios used. These can have different representation style and are defined as follow:

- Story: Narrative description of connected sequence of events, e.g. a user story that is written in plain text as often seen in agile methodologies. 
- Sequence: straight-line of interactive steps taken by human or system agents, e.g. List of numbered user actions.

- Structure: More elaborated representation of a scenario, e.g. activity diagram.

- Situation: Snapshot of a future state of the system, e.g. a picture or an example of a user interface of an imagined future state.

- Simulation: Models to explore and animate stories or situations, e.g. animated diagram to show the eventual real effects of alternative conditions and courses of action.

- Storyboard: Drawing or a sequence of drawings to describe a story, e.g. mock-ups of a flow that are linked together.

Natural language-based scenarios like use case (Cockburn, 2001) or scenario (Leite at al., 2000) representations, are widely used to specify software requirements because they promote the communication between engineers and stakeholders, even when they have no modeling background. Furthermore, natural language-based scenarios offer several practical advantages: (1) Scenarios are easy to describe and understand; (2) They are scalable; the behavior of a large and complex system can be represented as a collection of independently and incrementally developed scenarios; and (3) It is relatively easy to provide traceability throughout the design (Lee et al., 1998).

\subsubsection{3. \\ Use Case Representation}

A typical use case (Cockburn, 2001) describes the interaction (triggered by an external actor in order to achieve a goal) between a system and its environment. Every use case constitutes a goal-oriented set of interactions between external actors and the system under consideration. The term actor is used to describe any person or system that has a goal in the system under discussion or interacts with the system to achieve some other actor's goal. A primary actor triggers the system behavior in order to achieve a certain goal. A secondary actor interacts with the system but does not trigger the use case.

A use case is completed successfully when the goal that is associated with it is reached. Use case descriptions also include possible extensions to this sequence, e.g., alternative sequences that may also satisfy the goal, as well as sequences that 
may lead to failure in completing the service in case of exceptional behavior, or some fault. In the textual notation proposed by Cockburn (2001), the main flow is expressed, in the "description" section, by an indexed sequence of NL sentences, describing a sequence of actions of the system. Variations are expressed (in the "extensions" section) as alternatives to the main flow, linked by their index to the point of the main flow from which they branch as a variation (See Table 1).

Table 1 - Use Case Template (Cockburn, 2001)

\begin{tabular}{|c|c|c|}
\hline Element & \multicolumn{2}{|c|}{ Description } \\
\hline Use Case \# & \multicolumn{2}{|r|}{$<$ The name is the goal as a short active verb phrase $>$} \\
\hline Goal in Context & \multicolumn{2}{|r|}{$<\mathrm{A}$ longer statement of the goal in context if needed $>$} \\
\hline $\begin{array}{l}\text { Scope \& } \\
\text { Level }\end{array}$ & \multicolumn{2}{|r|}{$\begin{array}{l}<\text { What system is being considered black box under design }> \\
<\text { One of: Summary, Primary task, Sub-function }>\end{array}$} \\
\hline Preconditions & \multicolumn{2}{|r|}{$<$ What we expect is already the state of the world $>$} \\
\hline $\begin{array}{l}\text { Success } \\
\text { Condition }\end{array}$ & \multicolumn{2}{|r|}{$<$ The state of the world upon successful completion $>$} \\
\hline Failed End Condition & \multicolumn{2}{|c|}{$<$ The state of the world if goal abandoned $>$} \\
\hline $\begin{array}{l}\text { Primary, } \\
\text { Secondary Actors }\end{array}$ & \multicolumn{2}{|r|}{$\begin{array}{l}<\text { A role name or description for the primary actor }> \\
<\text { Other systems relied upon to accomplish the use case }>\end{array}$} \\
\hline Trigger & \multicolumn{2}{|r|}{$<$ The action upon the system that starts the use case $>$} \\
\hline \multirow[t]{2}{*}{ Description } & Step & Action \\
\hline & & $\begin{array}{l}<\text { Put here the steps of the scenario from trigger to goal delivery, and } \\
\text { any cleanup after }>\end{array}$ \\
\hline \multirow[t]{2}{*}{ Extensions } & Step & Branching Action \\
\hline & & $\begin{array}{l}<\text { Condition causing branching }> \\
<\text { Action or name of sub-use case }>\end{array}$ \\
\hline \multirow[t]{2}{*}{ Sub-Variations } & Step & Branching Action \\
\hline & & $<$ List of variations $>$ \\
\hline
\end{tabular}

\subsubsection{4.}

\section{Scenario Representation}

The scenario language proposed by Leite et al. (2000) describes situations in the system and its relation with other situations and the environment. This description is made using natural language. The proposed structure of this model is composed of the following elements: title, goal, context, resources, actors, episodes, exceptions and constraints (See Table 2). In the episodes, the operational behavior of the situations is described in natural language, but using special operators for optionality, concurrency and selection. A scenario is identified by a title and must satisfy a goal. The path to achieving this goal must be described in detail in its episodes. The episodes represent the main stream of the actions, but also include variations and possible alternatives. An exception can occur during the execution of episodes, which indicates that there is an obstacle to satisfy the goal. The treatment to this exception does not need to satisfy the scenario goal. 
Table 2- Scenario template (Leite et al., 2000)

\begin{tabular}{|c|c|}
\hline Element & Description \\
\hline Title & $<$ Identifies the scenario $>$ \\
\hline Goal & $<$ Describe the purpose of the scenario $>$ \\
\hline Context & $\begin{array}{l}<\text { Describes the scenario initial state }> \\
<\text { Must be described through at least one of these options: precondition, geographical } \\
\text { or temporal location }>\end{array}$ \\
\hline Resources & $<$ Passive entities used by the scenario to achieve its goal $>$ \\
\hline Actors & $<$ Active entities directly involved with the situation $>$ \\
\hline Episodes & $\begin{array}{l}<\text { Sequential sentences in chronological order with the participation of actors and use } \\
\text { of resources }> \\
<\text { One of: Simple, Conditional, Optional }> \\
<\text { Non-sequential order can be bounded by the symbol "\#”, it is used to describe } \\
\text { parallel or concurrent episodes }>\end{array}$ \\
\hline Exception & \begin{tabular}{|l|l|} 
Cause & Solution \\
\end{tabular} \\
\hline & $\begin{array}{l}<\text { Situations that prevent the proper course }<\text { Its treatment should be described }> \\
\text { of the scenario }>\end{array}$ \\
\hline
\end{tabular}

The attribute constraint is used to describe non-functional aspects that may restrict the goal of a scenario to be achieved within the desired quality. These nonfunctional aspects can be related to context, resources and episodes

The existence of relationships among scenarios is an important characteristic of this representation. Scenarios can be connected to other scenarios through links, yielding a complex network of relationships. These links can be of four distinct types: constraint, precondition, sub-scenario and exception. A constraint as well as a precondition can be described by another scenario. Subscenario or exception relationships are defined when an episode (sentence) or exception (solution) of a scenario is detailed in another scenario.

\subsection{4.}

\section{Natural Language-based Scenario Representations Compared}

There is no clear and correct answer when it comes to selecting the right representation or language for writing scenarios. The selection of appropriate technique depends on different factors and can be different for every project. Most of the existing languages or templates for writing use cases are extensions based on textual use case template proposed by Cockburn (2001). Therefore, most of the existing use case templates only represent specific situations between the user and the system through user interface. Other drawbacks of use cases based on Cockburn (2001) template are the following:

- Lack of precise definition, which originated that several companies have reinvented their own versions (Lee et al., 1998; Sinnig et al., 2009). 
- Consider only the user interactions with the system.

- The relationships among use cases are rarely explicit.

On the other hand, the scenario language proposed by Leite et al. (2000) represents situations in the domain application. A situation describes the interactions among actors in the Universe of Discourse, including interactions with a software system (existing or a future one) or the internal behavior of the application. Besides in Leite et al. (2000), are presented powerful characteristics to make explicit the relationships among different scenarios.

Table 3 - Scenario and Use Case Comparison

\begin{tabular}{|l|l|}
\hline Scenario (Leite et al., 2000) & Use Case (Cockburn, 2001) \\
\hline Title & Use Case \# \\
\hline Goal & Goal in Context \\
\hline Context & Scope \& Level \\
\cline { 2 - 2 } & Preconditions \\
\cline { 2 - 2 } & Success End Condition \\
\cline { 2 - 2 } & Failed End Condition \\
\hline Resources & Not applicable \\
\hline Actors & Primary, Secondary Actors \\
\hline Episodes & Trigger \\
\hline Exception & Description \\
\hline & Extensions \\
\cline { 2 - 2 } & Sub-Variations \\
\hline
\end{tabular}

Table 3 compares the elements that compose the scenario language (Leite et al., 2000) and use case representation (Cockburn, 2001). Scenario title and goal are equivalent to Use Case name and goal, respectively. Scenario context can be equivalent to Use Case scope \& level, pre-conditions and conditions. Scenario episodes are equivalent to use case description element. Scenario exception can be represented by use case extensions or sub-variations because they are triggered by situations that prevent the main course of actions. Scenario resources element is not applicable to use cases because use case does not consider this element.

\section{2.}

\section{Quality in Software Requirements Specification}

Software quality is defined by the IEEE as "the degree to which a system, component or process Answer: (1) the specified requirements, and (2) the expectations or needs of customers or users". On the other hand, the ISO defines quality as" the totality of characteristics of a product or service that demonstrate their ability to meet needs specified or implied". Therefore, these two definitions 
show that the quality of a software product is closely linked to meeting their requirements.

In requirements engineering, requirements quality is not just about whether the functionality has been correctly documented (Quality Assurance), but also depends on non-functional requirements.

\subsection{1. \\ Non-functional Requirements (NFR)}

Non-functional requirements (NFRs) are often called quality attributes of a system. Other terms for non-functional requirements are constraints, quality goals, quality of service requirements and non-behavioral requirements. In contrast to functional requirements, non-functional requirements define how a system should behave. They significantly influence the product quality of the final software system (Sommerville, 2010).

Quality attributes are hard to specify and are usually stated informally. In Goal-Oriented Requirements Engineering approaches (GORE), non-functional requirements are represented as soft-goals, whose satisfaction cannot be established in a clear-cut sense. The main objective of GORE is to iteratively refine higher-level requirements until concrete system requirements are obtained. The NFR framework developed by Chung et al. (2000) is a goal-oriented approach to represent non-functional requirements.

\subsubsection{1. \\ NFR Framework}

The NFR Framework (Chung et al., 2000) is a Goal-Oriented RE approach for capturing NFRs in the domain of interest, and defining their interdependencies and operationalizations. The NFR Framework allows to: (1) model the NFRs and their decomposition, (2) design alternatives for different NFRs, (3) deal with conflicts, tradeoffs, and priorities, and (4) evaluate the decisions impact centered on NFRs. These NFRs are modeled using a Soft-goals Interdependency Graph (SIG). The SIG graphically represent NFRs as soft-goal nodes (clouds); their refinements using AND/OR decompositions links; their positive/negative interdependencies as some+ (help), some- (hurt), some++ (make), some-- (break) contribution links; their operationalizations as leaf nodes; and claims as annotations in natural language. Generally, soft-goals are named using the 
convention Type [Topic1, Topic2...] where Type is the soft-goal and Topic is the field of application of Type; Topic is optional.

Figure 3 illustrates a very simple SIG that models the Software Requirements Specification Correctness, by considering its contributions - HELP links - in Specification Consistency, Completeness and Unambiguity. An interdependency between Consistency and Completeness negatively impacts (HURT) on both, because increasing Completeness might negatively impact Consistency. According to Glinz (2000), and Zowghi and Gervasi (2003), there is an important causal relationship between Consistency, Completeness and Correctness.

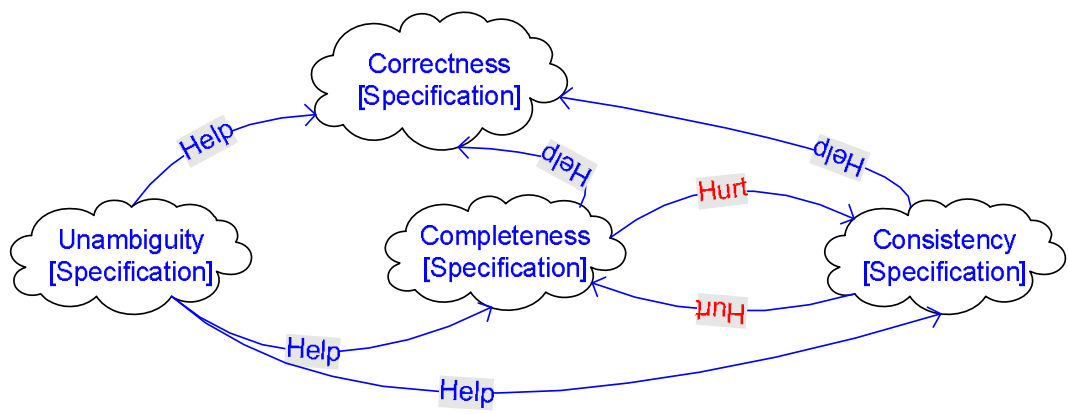

Figure 3 - SIG of Correctness.

\subsection{2.}

\section{Quality Assurance for Software Requirements}

Evaluating the quality of a SRS involves aspects related to quality assurance of software artifacts produced in the requirements engineering process. The quality of a requirements artifact can be addressed by the use of metrics, standards, prototyping, indicators or tests. A critical review of these artifacts may be accomplished through inspections.

Various authors have worked on quality assurance of software requirements. Some focus on the classification of quality into characteristics (Gnesi et al., 2005; Wilson et al., 1997; Lucassen et al., 2015; Arora et al., 2015), and the definition of quality models and metrics to quantify the presence of evidences (or not) of these characteristics; others developed comprehensive checklists or constructive approaches (Glinz, 2000; Leite et al. 2000; Denger et al., 2005; Phalp et al., 2007; Sinha et al., 2010) for detecting defects classified into taxonomy of defects.

Some researches focused in the automatic detection of defects in requirements (Gnesi et al., 2005; Arora et al., 2015; Sinha et al., 2010), taking advantage of NLP techniques. 
According to Gnesi et al. (2005), a Quality Model is the formalization of the definition of the term "quality" to be associated to a type of work product. The typical objectives of a quality model are to define, analyze, and document a product's:

- Quality Characteristics: define and document the relevant quality factors (also known as quality attributes or "ilities") that are important attributes of work products (e.g. applications, components, or documents) or processes that characterizes part of their overall quality (e.g. extensibility, operational availability, performance, re-usability, ...). Quality subcharacteristics are important properties of quality characteristics.

- Quality Indicators: are specific descriptions of something that provides evidence either for or against the existence of a specific quality characteristic or sub- characteristic.

- Quality Metrics: provide numerical values estimating the quality of a work product or process by measuring the degree to which it possesses a specific quality characteristic.

\subsubsection{1. \\ Software Requirements Quality Characteristics}

Most of the quality models or checklist for software requirements specifications (detailed in Section 2.5) are based on the quality characteristics defined by the IEEE std 830-1998 standard (IEEE, 1998). Among others, the desirable characteristics of a "good" SRS are:

- Complete: An SRS is complete if, most of relevant requirements are present and each requirement is fully developed (Boehm, 1979). It must not include situations that will not be encountered or capability features that are unnecessary (Wilson et al., 1997).

- Consistent: An SRS is consistent when, two or more requirements are not in conflict with one another (Boehm, 1979).

- Correct: An SRS is correct if and only if, every requirement stated therein is one that the software shall meet (IEEE, 1998).

- Unambiguity: An SRS is unambiguous if and only if, every requirement stated therein has only one interpretation (IEEE, 1998). Unambiguity 
requires the specification to be as formal as possible; however, in the vast majority of requirements specifications, requirements are stated informally with natural language or at best semi-formally. Thus, unambiguity is very difficult to achieve. (Glinz, 2000).

The ISO 29148 (IEEE, 2011) standard was created to harmonize a set of existing standards or quality models, including the IEEE 830-1998 (IEEE, 1998) standard. It differentiates between quality characteristics for a set of requirements, such as completeness or consistency, and quality characteristics for individual requirements, such as ambiguity or singularity. Apart from the "dos", it also provides some "don'ts" regarding requirements language (e.g. avoid ambiguous adverbs, vague pronouns, subjective language, and so on). Some researches classify these "don'ts" as indicators or smells of ambiguity (Wilson et al., 1997; Gnesi et al., 2005; Tjong, 2008; Femmer et al., 2014).

In practice, most software requirements specifications do not meet these quality characteristics. Thus, it is impossible to be complete as well as to assure correctness due to the completeness fallacy (Leite, 2007).

\subsubsection{2. \\ Verification \& Validation}

The terms Verification and Validation are commonly used in software engineering to mean two different types of analysis. According to Boehm (1979), the usual definitions are:

- Verification: to establish the truth of the correspondence between a software product and its specification, i.e. are we building the product right?

- Validation: to establish the fitness or worth of a soft-ware product for its operational mission, i.e. are we building the right product?

In other words, validation is concerned with checking that the software meets user's actual needs, while verification is concerned with whether the software is well-engineered, error-free, and so on. Verification helps to determine whether the software is of high quality, but it does not ensure that it is useful (Easterbrook, 2010).

Verification is a relatively objective process. It includes the activities associated with producing high quality software: inspection, analysis, simulation 
or checklist heuristics for evaluating that specifications are expressed precisely enough.

In contrast, validation is a subjective process. It should confirm that the Universe of Discourse situations, occurrences, have been reported in accordance with the real world needs of the users. Requirements validation includes techniques such as mock-up, storyboards and high level prototyping, and must be performed with clients and users.

A SRS can be verified by the following approaches: (1) inspection, to examine carefully and critically, especially for flaws; (2) analysis, a series of logical deductions based on logic or math oriented representations; (3) simulation, execution of a model, usually with a computer program; (4) checklist, an examination of the SRS by pre-defined rules, patterns or taxonomies.

Each of the above mentioned approaches for SRS verification has its own advantages and drawbacks. Formal verification through model checking techniques can be used for analysis of structural and behavioral properties; however, this approach is not well suitable for models with large number of states (the complexity of the generated reachability graph is exponential).

\subsubsection{3. \\ Quality of Scenarios}

Many authors have suggested using guidelines for writing use case descriptions (e.g. Cockburn, 2001; Denger et al., 2005; Phalp et al., 2007) and such guidance is often entirely plausible. For example, Cockburn's (2001) recommendation of "subject...verb... direct object... prepositional phrase", appears to be particularly straightforward and intuitive. Although these structure guidelines are meant to aid composition, the ultimate goal is to improve the resulting description.

Similarly, other authors suggest to measure compliance with the guideline suggestions (e.g.; Anda and Sjøberg, 2002; Denger et al., 2005; Leite et al., 2005; Phalp et al., 2007; Sinha et al., 2010). Indeed, these proposals use inspection techniques by examination of scenario (or use case) descriptions, and find defects using checklists.

Leite et al. (2005) developed a scenario-based reading (inspection) technique to improve the qualities of scenarios. The technique allows identifying 
missing, incorrect, ambiguous, contradicting and overlapping information in scenarios. The output is a list of discrepancies, errors, and omissions (DEOs). The inspection process is divided into four steps: plan, prepare, meet and rework. Their method was applied in 9 different case studies and returned positive results.

It was difficult to find formal definitions of quality characteristics related to scenarios; most of the authors suggested the use of taxonomy of defects and checklists to find potential problems. Anda et al. (2009) improved a previous use case inspection technique (Anda and Sjøberg, 2002), by defining an initial model of quality attributes for UML use cases.

\section{3.}

\section{Concurrency}

Concurrent systems (i.e., ones where there is more than one process existing at a time) present characteristics such as non-deterministic and synchronization between processes. Web systems provide the most obvious examples of concurrent systems, which can be characterized as a system where there are a number of different processes being carried out at the same time.

According to Roscoe (1998), what all concurrent systems have in common is a number of separate processes which need to interact with each other. Therefore, the crucial thing which makes concurrent systems different from sequential ones is the fact that their processes interact with each other at the level of communication (Roscoe, 1998). To understand this point, one process communicates with another by a named communication channel or a shared resource (or variable).

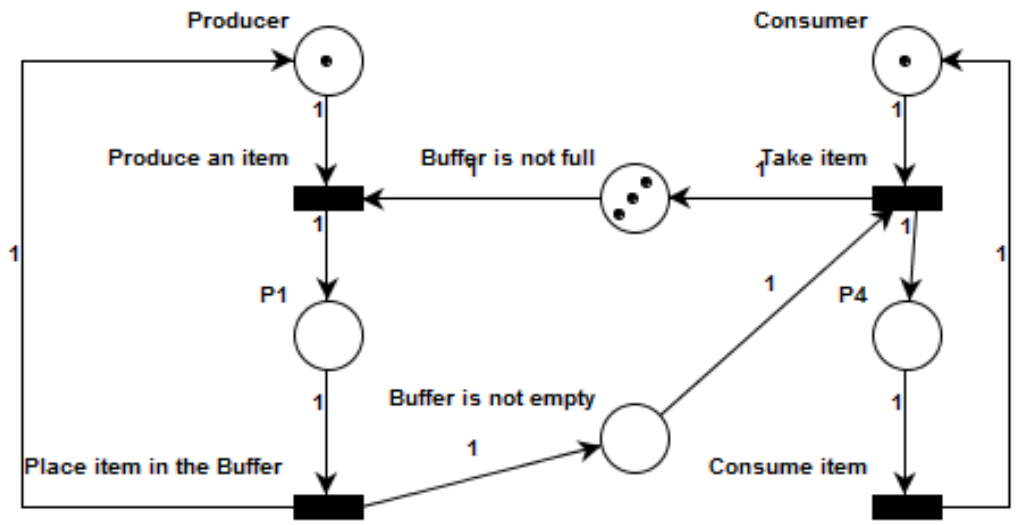

Figure 4 - Producer and Consumer Problem Using Petri-Nets

The Producer-Consumer problem is an example of communicating processes using a named channel called "the buffer"; the producer sends a 
message to the consumer putting an element in the buffer. Figure 4 shows this communication using a Petri-Net model.

In the Readers-Writers problem, processes communicate with each other by a shared resource; while the shared resource is being written or modified by the writer process, it is often necessary to bar other writer or reader processes. Figure 5 shows this communication using a Petri-Net model.

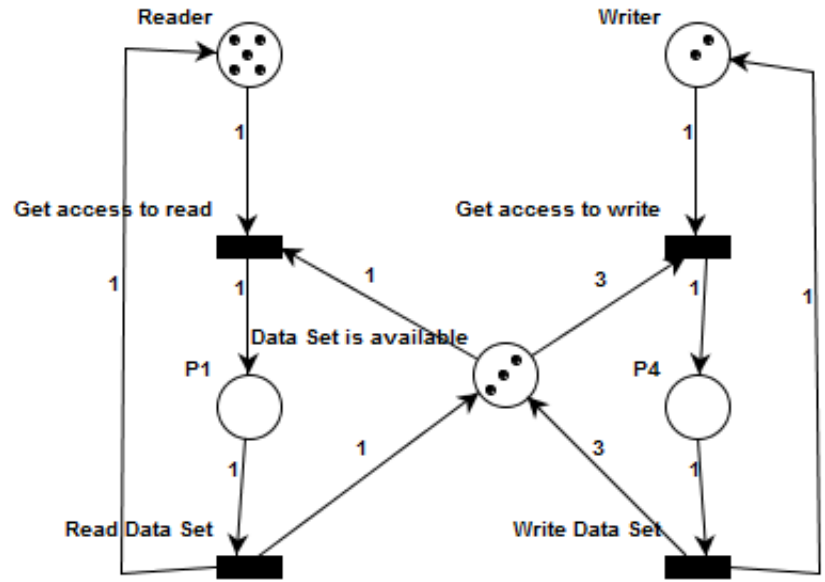

Figure 5 - Reader and Writer Problem Using Petri-Nets

\subsection{1.}

\section{Synchronization}

According to Downey (2005), Synchronization refers to relationships among several processes and any kind of relationships (before, during, after), i.e. it is related to the execution order of processes. Synchronization between two processes means that one process necessarily waits the other process. In sequential systems, computers execute one process after another, and it is possible to know the order of execution; but in concurrent systems it is impossible to tell. Examples of synchronization include: (1) Serialization - event A must happen before Event B; and (2) Mutual exclusion - events A and B must not happen at the same time.

\subsection{2.}

\section{Non-determinism}

A system exhibits Non-determinism if two different copies of it may behave differently when given exactly the same inputs (Roscoe, 1998). Concurrent systems are often non-deterministic because of interleaving accesses on shared resources, i.e. concurrent systems suffers from limited controllability and observability problem. 


\subsection{3. \\ Synchronization Constraints}

Due to non-deterministic behavior, it is difficult to ensure that the system is free of synchronization bugs. The most common alternative to reduce these bugs is the use of synchronization constraints to control concurrent access to shared resources. Serialization and Mutual exclusion are examples of synchronization constraints. Serialization may seem trivial, but the underlying idea, message passing, is a real solution for many synchronization problems (Downey, 2005).

The most common constraint is mutual exclusion, or mutex; mutex guarantees that only one process accesses a shared resource at a time, eliminating the kinds of synchronization bugs. Like serialization, mutual exclusion can be implemented using message passing (Downey, 2005).

\subsection{4.}

\section{Desired Properties of Concurrent Systems}

A system should employ the principles of modularity (top-down design) and make explicit the interconnectivity among modules. Modularity is considered as a mechanism to deal with the complexity of concurrent systems (Lee et al., 1998).

The design of concurrent systems is often modular and proceeds by first developing local processes, services, modules or components, and then composing these modules to one component. While the composing is performed using synchronization constraints, it is possible to introduce some concurrency bugs that impair the following desired properties of concurrent systems.

\subsubsection{1.}

\section{Deadlock-free}

A system should not have any deadlock situation. A concurrent system is deadlocked if no process can make any progress, generally because each is waiting for communication with others (Roscoe, 1998).

\subsubsection{2. Boundedness}

This property refers to the limited capacity of a communication channel or a shared resource (Murata, 1989). A concurrent system is overflowed when the number of elements in some channel or resource exceeds a finite capacity. 


\subsection{5. Petri-Net}

This section presents the fundamentals of Petri-Nets, especially of placetransition Petri-Nets (Reisig, 1985; Murata, 1989).

Petri-Net is a graphical and mathematical language for modeling and analysis of systems that are characterized as concurrent, asynchronous, distributed, parallel, nondeterministic, and/or stochastic. Due to these features, Petri-Nets can be used for modeling and analysis of: performance, communication protocols, distributed-software systems, distributed-database systems, concurrent and parallel programs, industrial control systems, discrete-events systems, multiprocessor memory systems, dataflow-computing systems, fault-tolerant systems.

They were introduced by Carl Adam Petri in 1962 at the Technische Universität Darmstadt, Germany.

A Petri-Net (Figure 6) is a directed, weighted, bipartite graph; and it is composed of nodes that denote places (Place) or transitions (Transition). Nodes are linked together by arcs (Arc).

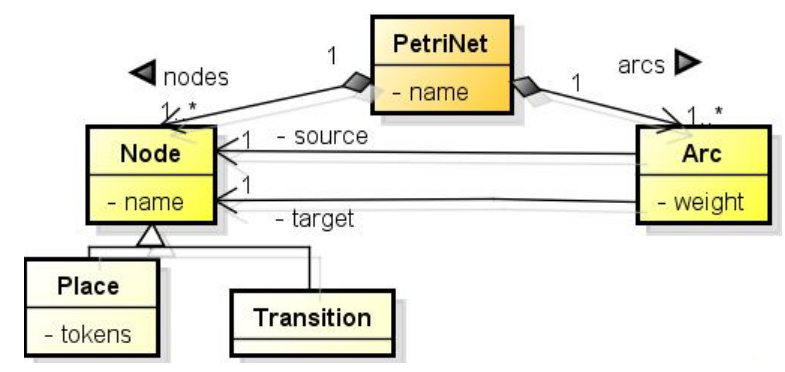

Figure 6 - Petri-Net metamodel (Sarmiento et al., 2015)

\subsubsection{1.}

\section{Petri-Net Definitions}

Transitions are active components. They model the activities that can occur, thus changing the state of the system. Transitions are only allowed to fire if they are enabled, which means that all the pre-conditions (input places) for the activity have been fulfilled.

Places are passive components and placeholders for tokens. They model communication medium, buffer, geographical location or a possible state (condition). The current state of the system being modeled is called marking, which is given by the number of tokens in each place. 
Tokens model physical or information object, collection of objects, resource availability, jobs to perform, flow of control, synchronization conditions, indicator of state or indicator of condition.

In addition, tokens are used in Petri-Nets to simulate the dynamic and concurrent activities of systems.

Arcs are of two types. Input arcs start from places and ends at transitions, while output arcs start at a transition and end at a place.

Definition 2.1. A place-transition Petri-Net (Reisig, 1985) is a five-tuple $\boldsymbol{P N}=\left(P, T, F, W, M_{0}\right)$ where $P=\left\{p_{1}, p_{2}, \ldots, p_{n}\right\}$ is a finite set of places, $T=\left\{t_{1}, t_{2}\right.$, $\left.\ldots, t_{m}\right\}$ is a set of transitions, $F \subseteq(P \times T) \cup(T \times P)$ is a set of arcs, $W: F \rightarrow\{1,2$, $\ldots\}$ is a weight function, $M_{0}: P \rightarrow\{0,1,2, \ldots\}$ is the initial marking and $P \cap T=$ $\varnothing$ and $P \cup T \neq \varnothing$.

In addition to have a static structure defined above, systems change over time and it is of great interest to study its dynamic behavior. In Petri-Nets, markings represent the states of the system over time. Figure 7 shows an example of a marked Petri-Net.

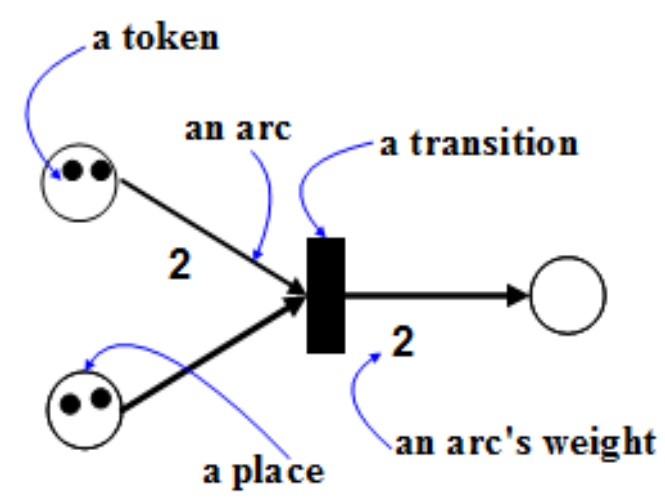

Figure 7 - Marked Petri-Net

Definition 2.2. For a $P N=\left(P, T, F, W, M_{0}\right)$, a marking is a function $M: P$ $\rightarrow\{0,1,2, \ldots\}$, where $M(p)$ is the number of tokens in $p . M_{0}$ represents $P N$ with an initial marking.

When a transition fires, it removes tokens from its input places and adds some at all of its output places. The number of tokens removed/added depends on the cardinality (weight) of each arc. Figure 8 shows an example of a marked PetriNet with a transition enabled for firing. 


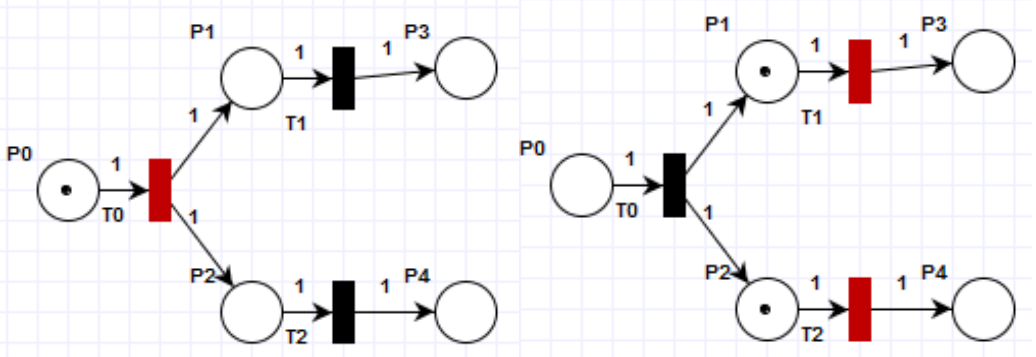

Figure 8 - (a) Transitions before Firing, (b) Transitions after firing

Definition 2.3. A transition $t$ is enabled for firing at a marking $M$ if $M(p) \geq$ $W(p, t)$ for any $p \in{ }^{\circ} t$ where ${ }^{o} t$ is the set of input places of $t$. On firing $t, M$ is changed to $M^{\prime}$ such that $\forall p \in P: M^{\prime}(p)=M(p)-W(p, t)+W(t, p) . M[t\rangle M^{\prime}$ denotes firing $t$ at marking $M . t^{o}$ is the set of output places of $t$. The notations ${ }^{o} p$ or $p^{o}$ have the same meaning for places.

Definition 2.4. For a $P N$, a sequence of transitions $\sigma=\left\langle t_{1}, t_{2}, \ldots, t_{n}\right\rangle$ is called a firing sequence if and only if $M_{0}\left[t_{1}\right\rangle,\left[t_{2}\right\rangle, \ldots,\left[t_{n}\right\rangle M_{n}$. In notation, $M_{0}[P N$, $\sigma) \underline{M_{n}}$ or $M_{0}[\sigma\rangle M_{n}$.

\subsubsection{2. Modeling with Petri-Nets}

In the real world, events may happen at the same time changing the state of a system over time. In systems modeled as Petri-Net models, the states of the system changes via enabling and firing transitions. A system may have many local states to form a global state.

Due to complex behavior of the systems, there is a need to model the relationships among several events of the system. In Petri-Net models, we may describe these relationships using sequential, non-deterministic, concurrency and synchronization structures.

In a sequential structure, events happen in a sequential order. In Figure 9, event $t 0$ fires before $t 1, t 1$ fires before $t 3$.

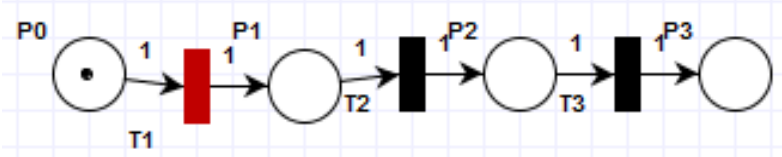

Figure 9 - Sequential structure

In a non-deterministic (conflict, choice, decision) structure, only one of the simultaneously enabled events may happen. In Figure 10, only one of event $t 1$ or t2 may fire. 


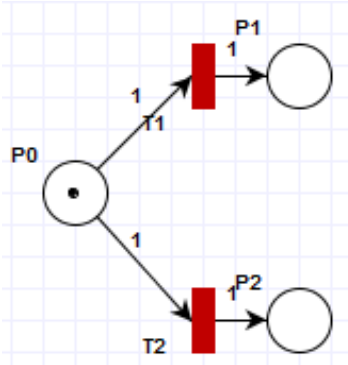

Figure 10 - Non-deterministic structure

In a concurrency structure, all simultaneously enabled events may happen. In Figure 11, events $t 1$ and $t 2$ may fire simultaneously.

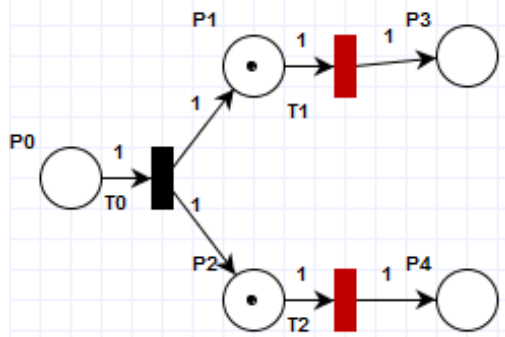

Figure 11 - Concurrency structure

In a synchronization structure, an event happens only if all events defined as pre-conditions or inputs happen. In Figure 12, event $t 3$ fires after events $t 1$ and $t 2$ fire.

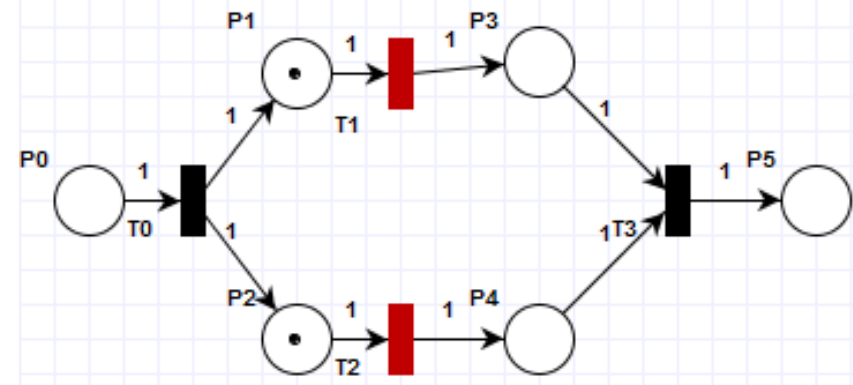

Figure 12 - Synchronization structure

In complex systems, several events happen at the same time and interact with each other. These relationships among events can lead to problems or erroneous situations such as deadlocks.

\subsubsection{3.}

\section{Analysis of Petri-Nets}

One feature that makes Petri-Nets interesting is that they also provide the capability to analyze model properties. The analysis of Petri-Net models evaluates defects related to structural and dynamic properties. The structural properties can 
be detected traversing the flow relation between places and transitions, while dynamic properties can be detected using the initial marking and markings which can be reached by firing transitions. Simulation, reachability/coverability or invariant analysis are methods for detecting defects due to dynamic properties like reachability, boundedness, liveness, and deadlock free (Reisig, 1985).

The most important analysis strategy is the reachability (Murata, 1989). Figure 13 shows that the marking of the Petri-net in Figure 13 (b) is reachable from the marking in Figure 13 (a).

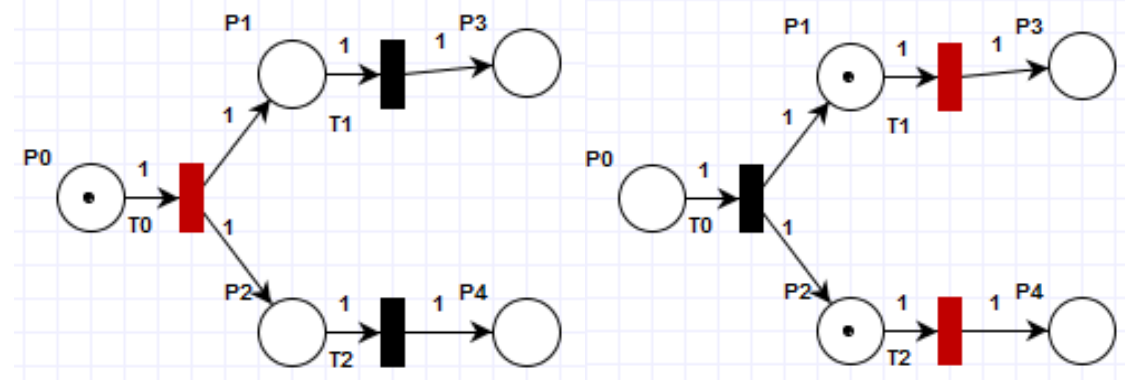

Figure 13 - (a) Transitions before Firing, (b) Transitions after firing

Definition 2.5. For a $P N=\left(P, T, F, W, M_{0}\right)$, a marking $M$ is said to be reachable if and only if there exists a firing sequence $\sigma$ such that $M_{0}[\sigma\rangle M$. In notation, $M_{0}\left[\mathrm{PN},{ }^{*}>\mathrm{M}\right.$ or $M_{0}\left[{ }^{*} / \mathrm{M}\right.$. represents the set of all reachable markings of $P N$.

If a Petri-Net is to be a model of a real hardware device, one of the important properties it should have is safeness. Safeness is a special case of the property called Boundedness.

Definition 2.6. A PN is bounded if the number of tokens in each place does not exceed a finite number $k$ for any marking reachable from $M_{0}$. A $P N$ is safe if it is 1-bounded.

Other important property is the concept of liveness (when modeling operating systems); and it is closely related to the complete absence of deadlocks (Murata, 1989).

Definition 2.7. For a $P N$, a transition $t$ is said to be live if it is possible to ultimately fire it by progressing through some firing sequence, i.e. if and only if $\forall M \in\left[M_{0}\right\rangle, \exists M^{\prime}: M\left[^{*} \mid M^{\prime}[t\rangle . P N\right.$ is said to be live if and only if every transition is live.

Definition 2.8. For a $P N$, if there exists a marking $M \in\left[M_{0}\right\rangle$ such that $\neg M$ [t> for any $t \in T$, then marking $M$ is called a dead marking of $P N$, i.e., a deadlock. 
A Petri-Net $P N$ is called deadlock-free if deadlock does not exist in PN. $\neg M[t\rangle$ denotes that $t$ is disenabled under $M$.

Another desirable property is that the system (when modeling manufacturing systems) can be re-initialized from any reachable state.

Definition 2.9. A $P N$ is reversible if $M_{0}$ is reachable from each other reachable marking $M$.

The reachability analysis method generates a reachability graph which contains reachable markings as nodes and transitions as arcs (which effect the change from one marking to another by firing). We can get an overview about possible states. Figure 14 shows the reachability graph for Petri-Net depicted in Figure 12 using the PIPE2 (2015) tool.
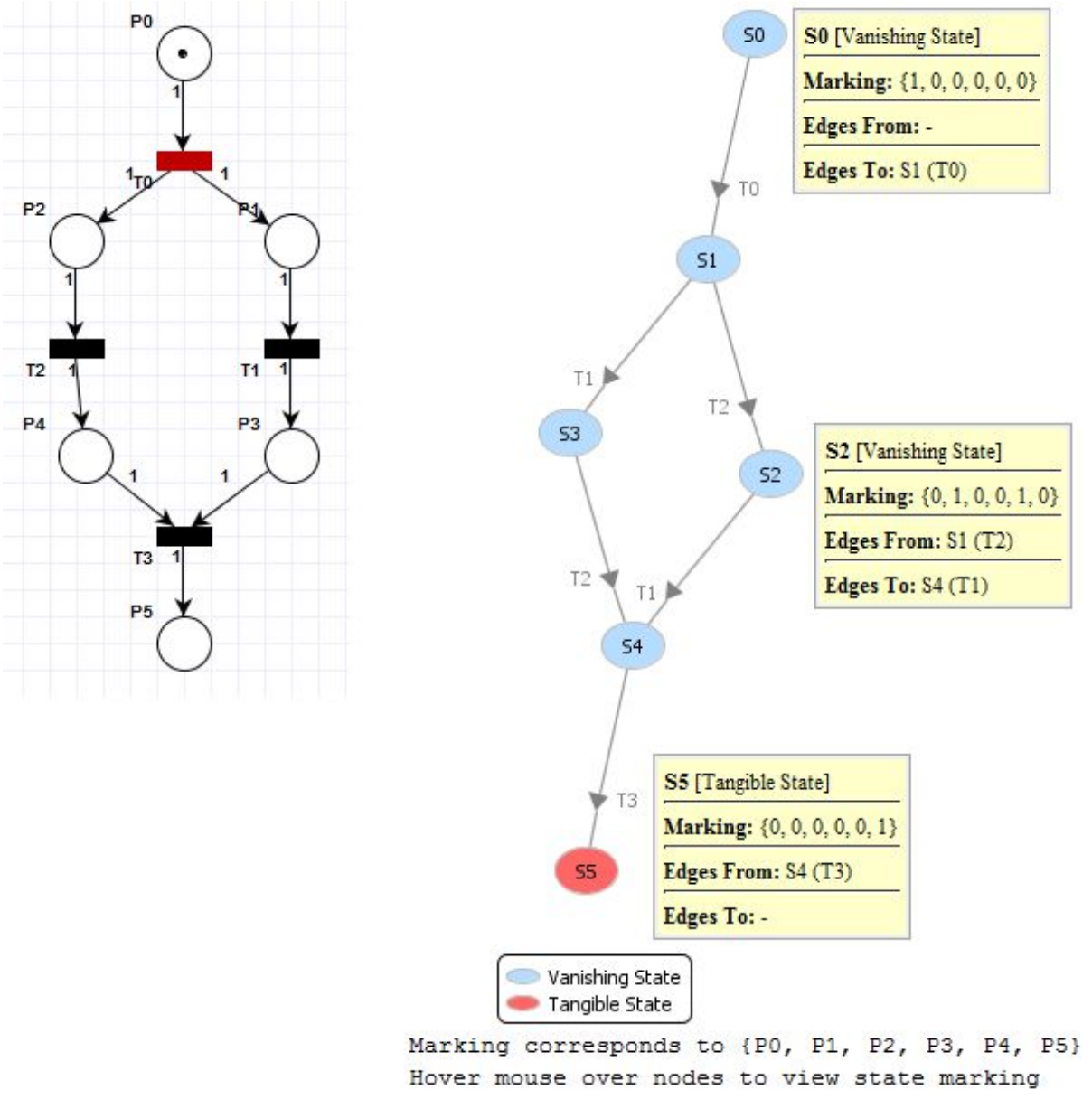

Figure 14 - A Reachable Petri-Net (generated using PIPE2, 2015)

In case of Petri-Nets with infinite many markings the computation of the reachability graph with this method fails. The state explosion issue is a serious problem when applying Petri-Net analysis to large systems, In fact, the generation of the reachability graph of a Petri-Net model requires an exponential space. Research continues to work on how to do it efficiently. 


\section{4 . \\ Considerations about Scenarios and Concurrency}

Such as introduced by Lee et al (1998) and Leite et al (2000), scenarios are rarely truly independent in practice; they may interact by complex relationships, what can lead to erroneous situations such as conflicts. Some of these relationships include potentially concurrent scenarios.

Considering that some languages for writing scenarios present characteristics for representing or identifying the relationships among different scenarios; they can be used to perform early concurrent system reliability assessment, i.e. scenarios can be used to detect potential concurrency defects at early software development activities.

Thus, scenarios are a key concept for writing software requirements, because: (1) they describe requirements such that users and developers can easily understand; (2) they may make explicit the relationships between scenarios, such as introduced by Leite et al (2000); and (3) they may support the early detection and resolution of quality defects.

Due to these characteristics, a scenario-driven requirements engineering approach has the potential to influence positively the Software Development process. Requirements engineers need to pay special attention to its quality, in special with respect to unambiguity, completeness, consistency and correctness since they will anchor further development.

\section{5 . \\ Related Work}

This sub section: (1) discusses research (or tools) related to the analysis of Natural Language based Software Requirements Specifications; (2) compares techniques to analyze static and dynamic aspects related to quality attributes of requirements; and finally (3) research gaps are appointed.

\subsection{1.}

\section{Analysis of Software Requirements Specification}

Not all the aspects related to the analysis of $S R S$ quality can be addressed in the same way and with the same depth and with the same ease, because it depends not only on internal requirements specifications, but also on external 
specifications (domain information or other software artifacts) and actual user's satisfaction.

In fact, Correctness evaluation of SRS, i.e. the verification that the system to be constructed is correctly described by them, needs to be supported by more rigorous methods (Glinz, 2000), and it depends on actual user's needs satisfaction.

Fortunately, some properties related to static (structural) and dynamic (behavioral) aspects of SRS can be addressed without increasing the formalism level. These properties may be grouped into three principal groups: Unambiguity, Completeness and Consistency.

Several studies dealing with the evaluation and the achievement of quality in NL based SRS can be found in the literature. Most of them are based on the definition of taxonomy of common defects or checklists necessary to improve the main quality characteristics of software requirements (unambiguity, completeness, consistency and correctness). Effectively, checklists or taxonomy of defects are used to perform the analysis of NL requirements aiming to detect defects and collect metrics. We will discuss some literature that we consider to be of particular interest to our research.

\subsection{2.}

\section{Overview of the State of the Art}

Quality in NL requirements can be addressed through the use of two types of analysis techniques, or the combination of them:

- Static Analysis: Analysis of the style and content of individual requirements or a set of requirements. This is accomplished by verification of conformance to the representation language, and in some cases by NLP techniques that can make explicit ambiguous terms and styles.

- Dynamic Analysis: Analysis of the behavior of a set of requirements to identify inconsistency or incorrectness defects, such as conflicting or overlapping requirements.

\subsubsection{1.}

\section{Static Analysis of Software Requirements Specification}

Requirements written using natural language are usually hard to analyze, because natural language is by definition ambiguous. Consequently, the inherent 
ambiguity of natural language makes difficult the evaluation of properties related to completeness and consistency quality attributes.

Ambiguity analysis and checking for properties related to completeness and consistency in requirements are usually performed by different stakeholders (e.g., developers, testers, customers, project managers) through a tedious procedure of reading requirements documents and looking for defects. According to Gnesi et al. (2005), most of the defects found by inspection techniques are in fact simple linguistic defects.

In static analysis, it is not necessary to execute the requirements for searching defect indicators that hurt unambiguity and completeness. Static analysis techniques may provide support that can facilitate the work of requirements engineers for checking consistency.

Software requirements (user or system requirements) may be a set of statements or scenarios; thus, static analysis techniques may be applied on requirements described as statements or represented as scenarios, depending on the level of abstraction.

\subsubsection{1.}

\section{Static Analysis of Requirement Statements}

Requirement statements are high-level descriptions of system functionalities. Most of the existing studies about analysis of requirement statements are addressed for detecting ambiguity indicators within individual statements. Only a few works (Arora et al., 2015; Lucassen et al., 2015) were proposed for searching defect indicators that contributes to completeness (mainly, conformance to requirements templates).

Wilson et al. (1997) examine the quality evaluation of NL software requirements. This approach defined a quality model composed of quality attributes and quality indicators, and develops an automatic tool called Automated Requirement Measurement (ARM) to perform the analysis against the quality model aiming to detect defects and collect metrics by searching the SRS document for words or phrases that have been identified as quality indicators, e.g. weak phrases. The quality model is composed of desirable characteristics for requirements specifications (Complete, Consistent, Correct, Modifiable, Ranked, Traceable, Unambiguous and Verifiable); although most of these quality attributes 
are subjective, there are aspects that can be measured and are indicators of quality attributes.

Based on the analysis of a set of requirements specification documents of NASA projects, nine categories of quality indicators were established. These categories are related to the evaluation of individual specification statements and entire requirements documents. Individual indicators were identified by finding frequently used words, phrases, and structures of the selected documents that were related to quality attributes and could be easily identified and counted by computer programs. These individual indicators were grouped according to their indicative characteristics.

Table 52 (Appendix 2) shows the main categories of indicators (or properties) that hurt Unambiguity and, for each of them, a sub-set of indicators detected by the ARM tool.

Gnesi et al. (2005) define a quality model for Natural Language requirements specifications, composed of the three target qualities to be achieved (Expressiveness, Consistency and Completeness). They concentrate on Expressiveness-related issues, leaving consistency and completeness problems for further studies. It includes those quality characteristics dealing with the understanding of the meaning of the requirements by humans. Linguistic techniques were used to address the issues related to the Expressiveness because the lexical and syntactical levels provide means enough to obtain effective results.

Expressiveness quality model is composed of three quality characteristics (Unambiguity, Specification Completion and Understandability) to be evaluated by means of indicators. We understand that these three quality characteristics are related to Unambiguity main quality. Indicators are linguistic components of the requirements directly detectable and measurable on the requirements document (See Table 53 in Appendix 2) that hurt Unambiguity.

In this approach the Quality Analyzer for Requirements Specifications (QuARS) tool performs a lexical analysis (morphological analysis) of the requirements document to evaluate properties that hurt Unambiguity. Vagueness, Optionality, Subjectivity and Readability defects are detected based on the occurrence of special terms in the requirements or the number of elements in the sentences. To point out the other indicators, it performs a syntactical analysis 
because the knowledge of the syntactical structure of the sentences is required to detect an Implicitly, Weakness, and Multiplicity indicators.

Tjong (2008) proposes an approach for Avoiding Ambiguity in Requirements Specifications, which provides a set of guideline rules and an inspection checklist for writing less ambiguous requirements. The guideline rules and checklist were implemented in an experimental lexical analyzer tool called SREE. During the inspection of the NL Requirements, it notifies the user about the potential ambiguity in the document, leaving space for the user to act upon and disambiguate a truly ambiguous statement.

The SREE is based on an Ambiguity Indicator Corpus (AIC), which contains the corpus of indicators of potentially ambiguous keywords, key phrases, and symbols. Although it may not be possible to have an AIC that contains an indicator of every possible potential ambiguity due to the richness of NL, SREE allows its user to add new indicators to its AIC. There are two categories of AICs in SREE, the Original Indicator Corpus (OIC) and the Customized Indicator Corpus (CIC). The OIC contains ten categories of ambiguity indicators (corpi), each in a separate file and each named appropriately for the nature of the potential ambiguities indicated by its contents: Continuance, Coordinator, Directive, Incomplete, Optional, Pronoun, Plural, Quantifier, Vague, and Weak. Each of these corpi has its own list of indicators. SREE automatically loads these corpi into the AIC each time a user starts up SREE.

Table 54 (Appendix 2) shows some of these indicators of these corpi, organized as properties that hurt Unambiguity.

In essence, SREE is a lexical analyzer. When SREE finds a word in its input matching one of the indicators in its AIC, then SREE notifies its user by printing out a message describing the kind of potential ambiguity suffered by the word.

Femmer et al. (2014) study the application of light-weight static analyses to make instant checks on natural language requirements and detect bad smells. Based on the ISO/IEC/IEEE 29148 standard (ISO, 2011), they derive a set of 8 smells that indicate potential issues in requirements specifications: ambiguous adverbs and adjectives, vague pronouns, subjective language, comparative phrases, and so on (See Table 55 in Appendix 2). 
The authors implemented a prototype tool for detecting patterns that are signs of potential quality defects in requirements by using NLP techniques, such as, part-of-speech tagging, morphological analysis, and customized dictionaries.

However, the granularity of the smells identified by the tool is limited to individual sentences, disregarding duplicate functionality among documents.

Arora et al. (2015) present an automated and tool-supported approach for checking conformance to requirements templates (conform to the templates of Rupp's (Pohl and Rupp, 2011) and EARS (Mavin et al., 2009). The approach builds on a mature Natural Language Processing technique, known as text chunking. In this context, the approach builds an NLP pipeline for text chunking. To instantiate the pipeline, one needs to choose, for each step in the pipeline, a specific implementation from the set of existing alternative implementations.

In addition to checking template conformance, they use NLP for detecting and warning about several potentially problematic constructs, also called requirements smells (Femmer et al., 2014), that may be signs of vagueness or ambiguity in requirements statements. Table 56 (Appendix 2) lists and exemplifies several constructs that can be detected automatically.

Lucassen et al. (2015) propose a Quality User Story Framework with 14 different criteria (See Table 57 in Appendix 2) to assess the quality of user stories (conform to the format of Cohn, 2004). Furthermore, the researchers developed a conceptual model to improve the quality of raw user stories by exploring defects and deviations in user stories. They developed the Automatic Quality User Story Artisan (AQUSA) tool on a NLP technique, which chunks the text and exposes defects and deviations from good practice in user stories. Unlike most NLP tools for RE, and in line with Berry's notion of a dumb tool (Berry et al., 2012); the tool detects some defects with close to $100 \%$ recall and high precision; this is necessary to avoid that a human requirements engineer has to double check the entire requirements document for missed defects. Consequently, AQUSA can support only certain syntactical and pragmatic criteria in an effective manner.

\subsubsection{2.}

\section{Static Analysis of Scenarios}

Scenarios are described by a set of more detailed statements of the functionality to be performed. Usually, scenarios are written using natural 
language. In our work, scenario and use case descriptions are considered synonymous because they are described by similar components.

Most of the existing approaches for scenario-based representations analysis are focused on checking of properties related to completeness, i.e., checking the conformance to scenario templates or guidelines and the coherence among scenario components (e.g. actors, pre-conditions, main flow descriptions, alternatives or extensions).

Only a few works (Leite et al., 2000; Anda and Sjoberg, 2002; Sinha et al., 2010, Liu, 2015) focused on checking some properties from the relationships among different scenarios. In these approaches, related scenarios are explicitly referenced within statements described in scenario descriptions.

Anda and Sjoberg (2002) develop a taxonomy of defects in use case diagrams and use case descriptions (conform to the template of Cockburn, 2001) considering different stakeholders. The defects are divided into omissions, incorrect facts, inconsistencies, ambiguities and extraneous information. They proposed a checklist-based inspection technique for detecting such defects in: (1) actors and use cases of use case diagrams; and (2) flow of events, variations, relationships between use cases, triggers, pre-conditions and post-conditions of use case descriptions. Table 58 (Appendix 2) shows the defects detected by the inspection technique.

Leite et al. (2005) present a strategy for scenario inspections to be performed by requirements engineers as a verification process before validation with clients, and they show how inspections help software developers to better manage the production of scenarios. This strategy was designed to be integrated with a specific scenario construction process (Leite et al., 2000) and was applied to several case studies. The data collected regarding the types of problems support their claim that scenario inspections do improve scenario quality.

Table 59 (Appendix 2) shows a checklist with verification heuristics for looking for discrepancies, errors and omissions (DEOs) in scenarios and their relationships with other scenarios and the symbols of the Language Extended Lexicon (LEL). The LEL (Leite et al., 2000) registers symbols (words or phrases) that are peculiar to the application domain. The types of symbols are: Subject, Object, Verb and State. Most of the heuristics for checking may be automated by intelligent editors and verification agents. 
Phalp et al. (2007) describe a Use Case Description Quality Checklist that acts as a check on the quality of the written description for detecting defects in use case descriptions. They proposed a set of heuristics ( 7 Cs of Communicability) that organizes the ideas, comments and suggestions of related work into categories relevant for improving the communicability of use case descriptions, and consequently producing a coherent set of desirable use case qualities. Their results of one experiment indicate that when a checklist is used in inspections it is mostly errors in syntax that will be discovered because they are easier to find than semantic ones.

The $7 \boldsymbol{C s}$ quality model is proposed as a set of heuristics to evaluate internal elements of use case descriptions (See Table 60 in Appendix 2).

Ciemniewska and Jurkiewicz (2007) developed a method for detecting requirements defects in an automatic way using simple heuristics and NLP. This thesis focuses on requirements in a form of use cases, as they consist of simple structure sentences, which are easy to analyze with available Natural Language Processing tools. Defects are organized in three levels: at the level of specifications, use cases, and steps. The level of specifications considers the behavior duplication. The level of use cases considers use cases very short or long, or complex extensions (alternative flows), among others. The level of steps considers complex syntactic structures, or omission of actors, among others. Heuristics detect defects by counting sentences, searching keywords or terms stored in dictionaries. NLP tool is used for searching verbs, subjects and objects in sentences. Table 61 (Appendix 2) shows the defects detected by the automated method.

Sinha et al. (2010) present a tool-supported approach for inspection (during edit time) of use case models (conform to a use case description metamodel), in conjunction with models from associated use cases, and reports on problems found. Text2Test tool detects problems related to style and content of use cases performing a linguistic analysis. Domain specific knowledge is needed to introduce semantic information to the analysis, assigning classification confidence to the concepts and verbs (type of action) described in use case sentences.

Tex2Test runs a set of checks for looking for defects in use case models; however, not every condition of interest (check) for a use case can evaluated automatically, but the set of interesting conditions that can be evaluated 
automatically is quite large (if not open-ended). Some examples of these conditions are shown in Table 62 (Appendix 2), and some of which are of particular interest for test-case generation.

Liu et al. (2014) present a tool-supported approach to achieve automatic defect detection in use case documents by leveraging on advanced parsing techniques. In this approach, they first parse the use case document using dependency parsing techniques; the dependency parsing provides richer syntactic details, i.e., provides the subject, object and main verb information of a sentence directly. The parsing results of each use case are further processed to form an activity diagram. Lastly, they perform defect detection on the activity diagrams.

In order to find defects in a set of use cases, Liu (2015) proposed a heuristic for finding relationships among use cases by constructing Deterministic FiniteState Automaton (DFA) from the behavior in individual use cases and composing them in a whole use cases graph. Traversing this graph is possible to find potential missing scenarios or whether a certain pre-condition is satisfied by certain postcondition.

The heuristic for finding relationships among use cases depends on an active learning strategy; they discover missing scenarios by generating questions to users. They base their approach on common use case defects shown in Table 63 (Appendix 2).

\subsubsection{2.}

\section{Dynamic Analysis of Software Requirements Specification}

In order to improve the correctness and consistency of systems described as informal requirements (or semi-formal), it is necessary to perform an extensive and iterative analysis, which is mostly performed manually, requiring a great effort and taking a lot of time. Inexperienced inspectors often do not detect these defects or only with much effort. The other option is write requirements using formal languages, such as Petri-Nets (Murata, 1989), LTS (Keller, 1976) or CSP (Roscoe, 1998), which will allow an automatic and rigorous analysis, which usually reduces the effort and time to be done.

In order to evaluate dynamic aspects of requirements, it is necessary to execute (or simulate the execution) a set of requirements for detecting defects that can hurt properties related to consistency or correctness. The use of formal 
techniques make it possible to perform a rigorous analysis and improve the consistency and correctness of a set of requirements, i.e., the set of requirements contains less erroneous situations such as conflicts or overlaps raised from the complex relationships among requirements. There is an interaction (or relationship) when two or more requirements have some effect on each other.

Many researchers have shown the importance to formalize the informal aspects of requirements in order to benefit from automated analysis of dynamic aspects. Usually, these approaches describe requirements as scenario representations. Some research focused on developing formal syntax and semantics for scenario representations, like Hsia et al. (1994) and Cheung et al. (2006); others are focusing on developing techniques to transform scenarios into executable representations. Therefore, these researches demonstrate that informal or semi-formal requirements cannot be used for further automated analysis.

Due to the focus of this work is the analysis of scenarios written using natural language, we selected related studies that focus on natural language-based scenarios. A few of them are supported by full automatic tools.

Lee et al. (1998) propose a systematic procedure to formalize use cases, by mapping use case descriptions into Constraint-based Modular Petri-Nets (CMPNs), allowing the analysis of use cases. To facilitate the transformation, use cases are described in relation to formal definition of pre and post-conditions, and represented like Action-Condition tables. Use cases are considered as a collection of interacting and concurrently executing units of system functionalities. Petri-Net analysis techniques can be used to evaluate completeness and consistency related properties in CMPNs (See Table 64 in Appendix 2). It is the unique approach that manages the state explosion problem of Petri-Nets by dividing the CMPN into a set of slices. However, intermediate models are created and it uses a non-standard use case model without alternative/exception flows.

Lee et al. (2001) present an approach to analyze use cases using formal semantics of Time Petri-Nets. Use case are used to elicit system requirements; in order to represent the interaction between the actors and the system, scenarios are derived from these use cases and represented as sequence diagrams. From these sequence diagrams, Time Petri-Nets are derived to check the acquired scenarios by indicating missing information (incompleteness) or wrong information (inconsistency) hidden in these scenarios. The approach avoids deadlock 
situations in the mapping process because sequence diagrams are constrained by the time line. However, relationships among use cases are no considered. Table 65 (Appendix 2) shows the faults detected by a CASE support tool.

Denger et al. (2005) present an integrated approach for achieving high quality in use cases that combines Use Case creation guidelines, Use Case inspections, and simulation in a systematic way. They base their combined approach on a defect classification for use cases (Table 66 in Appendix 2). This classification enables the requirements engineer to focus the different techniques on different types of defects. They showed that guidelines are valuable for the prevention of structural and syntactic defects, and inspections are suitable for detecting subtle logical defects. Simulation is integrated so that serious consistency and correctness defects resulting from the interference between Use Cases can be efficiently detected; for it Use Case are mapped into Statecharts (Harel, 1987), and several statecharts can be simulated simultaneously.

Ad-hoc recommendations, guidelines and checklists are used for avoiding defects that hurt unambiguity and completeness; simulation is used for detecting defects that hurt consistency.

Sinnig et al. (2009) propose a syntax definition that formalizes the sequencing of use case steps and their types; based on these syntax a formal semantics based on Labeled Transition System (LTS) (Keller, 1976) is proposed for use case models containing extend and include UML relationships. A use case model is mapped to UC-LTS by generating UC-LTSs from use case descriptions (steps and extensions), and merging the UC-LTSs representing the various entailed use cases.

The authors developed the Use Case Analyzer tool to automatically detect livelocks. They also propose a method for verifying refinement of use case models, namely checking their equivalence and deterministic reduction. Most of the checks focus on global properties of use case models, and only sequential relationships (precedence) among use cases are considered (See Table 67 in Appendix 2).

Zhao and Duan (2009) propose an approach to formalize use cases semantics with Timed and Controlled Petri-Nets (TCPN). A semi-structured natural language is proposed for use case syntax. The events in use cases can be sequential, conditional, iteration or concurrent (parallelism). Petri-Nets are 
derived mapping use case events into sub Petri-Nets and linking them. Based on the obtained Petri-Net model, criteria to detect incompleteness, inconsistency and incorrectness properties are described (See Table 68 in Appendix 2). This approach evaluates properties of use cases and their associated use cases, separately; thus, relationships among use cases are no considered.

Somé (2010) proposes an approach for formalizing textual use cases via reactive Petri-Nets (Eshuis and Dehnert, 2003). They provided an algorithm for the generation of a reactive Petri-Net from textual use cases described using a formal syntax, and taking into account include and extend UML relationships and sequencing constraints using pre/post-conditions. The constructed reactive PetriNet can be used for synthesis or analysis of Consistency properties defined in the transformation are satisfied (Table 69 in Appendix 2). This approach deals with sequential UML relationships among use cases (include and extend). However, the language to describe use cases does not deal with communication between concurrent use cases and other type of relationships among use cases.

\subsection{3.}

\section{Analysis Approaches Compared}

The studies reported in the literature for analysis of requirement statements are focused mainly in analysis of static aspects of individual requirement sentences in order to detect and correct ambiguity issues. The evaluation of the different techniques were based on the following aspects: What requirement representation is used?, What analysis technique is used?, Are relationships among requirements considered for analysis?, Is it toolsupported?, Does it detect unambiguity defects?, Does it detect completeness defects?, Does it detect consistency defects?, Does it detect correctness defects?, Is it applicable to scenario representations?. Table 4 summarizes the results in a matrix.

Most of the reported techniques (Section 2.5.2.1.1) support the detection of ambiguity defects based on indicators databases and NLP techniques (except Wilson et al., 1997). Only Lucassen et al. (2015) consider the relationships among requirements for analysis. Lucassen et al. (2015) and Arora et al. (2015) check completeness in relation to conformance to requirements templates; Gnesi 
et al. (2005) and Lucassen et al. (2015) supports the consistency checks by making explicit related requirements.

Most of these techniques are applicable to analysis of requirements described as scenario representations, since analysis of ambiguity can be applied to individual scenario elements or steps.

Table 4 - Comparing Requirement Statements Static Analysis Techniques

\begin{tabular}{|c|c|c|c|c|c|c|}
\hline & $\begin{array}{l}\begin{array}{l}\text { Wilson et } \\
\text { al., } 1997\end{array} \\
\end{array}$ & $\begin{array}{l}\text { Gnesi et al. } \\
2005\end{array}$ & Гjong, 2008 & $\begin{array}{l}\text { Femmer et al. } \\
2014\end{array}$ & $\begin{array}{lll}\text { Arora et a } \\
2015\end{array}$ & . Lucassen et al., \\
\hline \begin{tabular}{|l|} 
Requirement \\
Representation \\
\end{tabular} & No & No & No & No & $\begin{array}{l}\text { Rupp's; } \\
\text { EARS; }\end{array}$ & User Story; \\
\hline Analysis Technique & \begin{tabular}{|l} 
Dictionary \\
of \\
Indicators;
\end{tabular} & $\begin{array}{l}\text { Dictionary of } \\
\text { Indicators; } \\
\text { NLP; }\end{array}$ & $\begin{array}{l}\text { Dictionary of } \\
\text { Indicators; } \\
\text { NLP; }\end{array}$ & $\begin{array}{l}\text { Dictionary of } \\
\text { Indicators; } \\
\text { NLP; }\end{array}$ & $\begin{array}{c}\text { Dictionary of } \\
\text { Indicators; } \\
\text { NLP; }\end{array}$ & $\begin{array}{l}\text { Dictionary of } \\
\text { Indicators; } \\
\text { NLP; }\end{array}$ \\
\hline $\begin{array}{l}\text { Relationships Among } \\
\text { Requirements }\end{array}$ & \begin{tabular}{l|l} 
& No \\
\end{tabular} & No & No & No & No & Yes \\
\hline Tool-supported & Yes & Yes & Yes & Yes & Yes & Yes \\
\hline Unambiguity & Yes & Yes & Yes & Yes & Yes & Yes \\
\hline Completeness & No & No & Partial & Partial & Yes & Yes \\
\hline Consistency & No & Partial & No & No & No & Partial \\
\hline Correctness & No & No & No & No & No & No \\
\hline Applicable to Scenario & Yes & Yes & Yes & Yes & Yes & Partial \\
\hline
\end{tabular}

Most of the studies reported in the literature for analysis of scenarios are focused mainly in analysis of static aspects of scenario elements. The evaluation of the different static analysis techniques were based on the following aspects: What scenario representation is used?, Is there a syntax for scenario?, What analysis technique is used?, Are relationships among internal components of scenarios considered (e.g. actors, steps, extensions) for analysis?, Are relationships among scenarios considered for analysis?, Is it tool-supported?, Does it detect unambiguity defects?, Does it detect completeness defects?, Does it detect consistency defects?, Does it detect correctness defects?. Table 5 summarizes the results in a matrix.

Most of the reported techniques support the detection of ambiguity indicators and completeness defects by applying inspection checklists; Ciemniewska and Jurkiewicz (2007), Sinha et al. (2010) and Liu et al. (2014) also take advantage of NLP techniques. Phalp et al. (2007) does not consider the relationships among scenarios for analysis. Only Ciemniewska and Jurkiewicz (2007), Sinha et al. (2010) and Liu et al. (2014) present tool-supported techniques.

Most of these techniques check completeness in relation to conformance to scenario templates. Leite et al. (2000) provides heuristics for consistency checking; others support partially the consistency checking, e.g., Ciemniewska 
and Jurkiewicz (2007) provide heuristics for automatic detection of use case duplication. Only Leite et al. (2000) checks the consistency between scenarios and domain information (LEL).

Most of these techniques do not provide support for analysis of dynamic aspects of scenarios, because they do not provide execution semantics or insights for mapping into executable models.

Table 5 - Comparing Scenarios Static Analysis Techniques

\begin{tabular}{|c|c|c|c|c|c|c|}
\hline & $\begin{array}{l}\text { Leite et al } \\
2000\end{array}$ & Andar an & $\begin{array}{l}\text { Ciemniewska a1 } \\
\text { Jurkiewicz, 2007 }\end{array}$ & $\begin{array}{l}\text { Phalp et al } \\
2007\end{array}$ & $\begin{array}{l}\text { Sinha et } \\
2010\end{array}$ & . $\begin{array}{l}\text { Liu et al } \\
2014\end{array}$ \\
\hline Scenario Representation & Scenario & $\begin{array}{l}\text { Use Case } \\
\text { Diagram; } \\
\text { Use Case; }\end{array}$ & Use Case & Use Case; & $\begin{array}{l}\text { Use Case } \\
\text { diagram; } \\
\text { Use Case; }\end{array}$ & Use Case; \\
\hline Syntax for Scenarios & Yes & Partial & Partial & Yes & Yes & Partial \\
\hline Analysis Technique & $\begin{array}{l}\text { Checklist; } \\
\text { Heuristics }\end{array}$ & Checklist & $\begin{array}{l}\text { Heuristics; } \\
\text { NLP; }\end{array}$ & Checklist & $\begin{array}{l}\text { Checklist; } \\
\text { NLP; }\end{array}$ & $\begin{array}{l}\text { Checklist; } \\
\text { NLP; }\end{array}$ \\
\hline $\begin{array}{l}\text { Relationships Among } \\
\text { Internal Components }\end{array}$ & Yes & Partial & Yes & Partial & Yes & Partial \\
\hline $\begin{array}{|ll|}\text { Relationships among } \\
\text { Scenarios }\end{array}$ & Yes & Partial & Partial & No & Partial & Partial \\
\hline Tool-supported & Manual & Manual & Yes & Manual & Yes & Yes \\
\hline Unambiguity & Partial & Partial & Yes & Partial & Partial & Partial \\
\hline Completeness & Yes & Yes & Yes & Yes & Yes & Partial \\
\hline Consistency & Yes & Partial & Partial & Partial & Partial & Partial \\
\hline Correctness & Partial & Partial & Partial & Partial & Partial & No \\
\hline
\end{tabular}

Most of the studies reported in the literature for analysis of dynamic aspects of scenarios are focused on translating natural language-based scenarios into formal representations, and take advantage of execution semantics of formal languages to simulate the behavior of set of scenarios and detect defects from possible interactions. The evaluation of the different dynamic analysis techniques were based on the following aspects: What scenario representation is used?, Is there a syntax for scenario?, What analysis technique is used?, Are relationships among internal components of scenarios considered (e.g. actors, steps, extensions)?, Are relationships among scenarios considered for analysis?, Are non-explicit relationships among scenarios considered for analysis?, Are related scenarios integrated for whole analysis?, Is the state explosion issue of reachability analysis managed?, Is it tool-supported?, Does it detect unambiguity defects?, Does it detect completeness defects?, Does it detect consistency defects?, Does it detect correctness defects?. Table 6 summarizes the results in a matrix.

Most of the reported techniques support partially the detection of unambiguity and completeness defects by applying inspection checklists; these 
checklists mainly evaluate the conformance to previous syntax defined. Lee et al. (2001), and Zhao and Duan (2009) do not consider the relationships among scenarios for analysis. Only Lee et al. (1998) take into account non-explicit relationships among scenarios for analysis. Most of the techniques that consider relationships among scenarios integrate the related scenarios for a whole analysis. Lee et al. (1998) and Glinz (2000) do not present tools to support the analysis.

Most of these techniques are based on mapping to formal representations to take advantage of execution semantics, such as Petri-Nets, LTS or Statecharts; from these representations, a reachability graph is generated for analysis of the behavior of a set of scenarios and their relationships.

Most of these techniques do not take into account the state explosion issue of the generated reachability graph. Only Lee et al. (1998) manages the state explosion issue of reachability analysis by the use of slices strategy.

Petri-Net based techniques represent the interaction among concurrent scenarios in a more intuitive way, by fusing places or transitions to show the communication among concurrent scenarios.

Most of these techniques check consistency and correctness using equivalent formal representations, and they rarely map the results of the analysis to scenarios or the scenario relationships.

Table 6 - Comparing Requirement Statements Dynamic Analysis Techniques

\begin{tabular}{|c|c|c|c|c|c|c|}
\hline & Lee et al., 1998 & $\begin{array}{l}\text { Lee et al., } \\
2001\end{array}$ & $\begin{array}{l}\text { Denger } \\
\text { al., 2005 }\end{array}$ & $\begin{array}{l}\text { et Zhao } \\
\text { Duan, } 2009\end{array}$ & \begin{tabular}{l|l} 
and $\mid \begin{array}{ll}\text { Sinnig } \\
\text { al., 2009 }\end{array}$ \\
\end{tabular} & $\begin{array}{l}\text { et Somé, } \\
2010\end{array}$ \\
\hline Scenario Representation & $\begin{array}{l}\text { Use Case; } \\
\text { Action-Condition } \\
\text { table; }\end{array}$ & $\begin{array}{l}\text { Use case; } \\
\text { Sequence } \\
\text { Diagram; }\end{array}$ & Use Case; & Use Case; & $\begin{array}{l}\text { Use Case; } \\
\text { Use Case } \\
\text { diagram; }\end{array}$ & $\begin{array}{l}\text { Use Case; } \\
\text { Use Case } \\
\text { diagram; }\end{array}$ \\
\hline Syntax for Scenarios & No & Partial & Yes & Yes & Yes & Yes \\
\hline Analysis Technique & $\begin{array}{l}\text { Constraints-based } \\
\text { Modular Petri- } \\
\text { Net; }\end{array}$ & $\begin{array}{l}\text { Time Petri- } \\
\text { Nets; }\end{array}$ & $\begin{array}{l}\text { Checklist; } \\
\text { Statechart; }\end{array}$ & $\begin{array}{l}\text { Timed } \\
\text { Controlled } \\
\text { Petri-Nets; }\end{array}$ & and LTS; & $\begin{array}{l}\text { Reactive } \\
\text { Petri-Net; }\end{array}$ \\
\hline \begin{tabular}{|l|} 
Relationships Among \\
Internal Components \\
\end{tabular} & No & Yes & Partial & Partial & Yes & No \\
\hline $\begin{array}{|ll|}\begin{array}{l}\text { Relationships among } \\
\text { Scenarios }\end{array} & \\
\end{array}$ & Yes & No & Partial & No & Yes & Partial \\
\hline \begin{tabular}{|l|} 
Non-explicit \\
Relationships among \\
Scenarios
\end{tabular} & Yes & No & No & No & No & No \\
\hline \begin{tabular}{|l} 
Integration of Related \\
Scenarios for Whole \\
Analysis
\end{tabular} & Yes & No & Partial & No & Partial & No \\
\hline Tool-supported & No & Partial & Partial & Partial & Partial & Yes \\
\hline $\begin{array}{|ll|}\text { State } & \text { Explosion } \\
\text { Management } & \end{array}$ & Slices & No & No & No & No & No \\
\hline Unambiguity & Partial & Partial & Partial & Partial & Partial & Partial \\
\hline Completeness & Yes & Partial & Partial & Partial & Partial & Partial \\
\hline Consistency & Yes & Partial & Partial & Partial & Partial & Partial \\
\hline Correctness & Yes & Partial & Partial & Partial & Partial & Partial \\
\hline
\end{tabular}




\subsection{4.}

\section{Research Gaps}

Due to the focus of our research is the analysis of requirements described as scenario representations, we have identified the following gaps in scenariobased analysis techniques:

- We have not seen an explicit understanding of how the main quality characteristics are related to each one, i.e. they have positive and negative contributions among them. Only the requirement statements analysis works decomposed the main qualities (Unambiguity) in related properties (Categories of indicators) and modeled their impacts.

- Most of the techniques for static or dynamic analysis of scenarios do not take into consideration the results achieved by requirement statements analysis techniques in finding ambiguity indicators. Mainly, the works of Gnesi et al. (2005), Tjong (2008) and Lucassen et al. (2015) demonstrate that NLP techniques may be effective in detecting ambiguity, in some cases with $100 \%$ recall and high precision.

- Most of the analysis techniques are applied on scenarios written using formal syntax rules, or based on purely textual descriptions (conform to the template of Cockburn, 2001). Therefore, there is a lack of systematic procedures on how to represent scenarios. In case of techniques based on Cockburn (2001), intermediate models are created for mapping into formal representations.

- Only few of the works, like Somé (2010) apply consistency rules for verifying the preservation of the consistency between scenarios and their equivalent formal representations.

- Scenarios are rarely independent; they interact, in some cases nonexplicitly. Most of the existing proposals only consider sequential relationships (e.g. extend or include UML relationships) among scenarios; they do not propose constructs or heuristics to identify non-explicit relationships. Non-explicit relationships can hide non-sequential interactions (indistinct sequential order, concurrency or parallelism) among scenarios. 
- Most of the existing techniques do not describe systematic procedures on how integrate the equivalent formal representations of a set of related scenarios (they interact) into a whole representation, in order to evaluate the behavior and detect defects from the relationships in a set of scenarios. Only Lee et al. (1998) present a formal approach to integrate formal use cases with sequential and non-sequential (concurrent) relationships. Sinnig et al. (2009) and Somé (2010) give some details for the integration of sequentially related scenarios.

- The state explosion issue is a big problem when reachability graphs are generated for analysis of complex systems. Only Lee et al. (1998) propose a strategy to lead with this problem.

- When equivalent formal representation are evaluated using formal analysis strategies, and errors are found, the results of the analysis must be described in a comprehensive way, and mapped to defects within scenarios or the scenarios' relationships. None of the existing approaches return a feedback in a comprehensive way and tracing the errors from formal representations to scenarios. 


\section{A Quality Model for Scenarios}

This section begins with a general introduction of Software Requirements Specification (SRS) quality and the importance of scenario-based SRS (Section 3.1). Next, In Section 3.2 we employ the non-functional requirements (NFR) approach (Chung et al., 2000) to model the relationships between unambiguity, completeness, consistency and correctness qualities. This section also shows how a quality property can be evaluated by searching defect indicators. Finally, Section 3.3 discusses the benefits of the proposed Quality Model for Scenarios.

\section{1.}

\section{Quality in Scenario-based SRS}

Many problems and high-risk issues that arise during the software development process are related with deficiencies at RE activities. Assessing that SRS satisfices (relative satisfaction) the necessary quality is crucial to the success of any software development project, since the SRS is the anchor for software development. However, assessing the quality of a SRS is not a simple process, mainly, because a system must often support multiple stakeholders with different viewpoints and needs, which may be contradictory.

Nowadays, Scenario-based representations are frequently used in RE for requirements specification. A scenario-based SRS has the potential to influence positively the SD process, requirements engineers need to pay special attention to its quality, in special with respect to unambiguity, completeness, consistency and correctness since they will anchor further development.

There are several different templates or syntax for writing scenarios, and most of common components used to detail scenarios are:

- Title/Name: Name that identifies the scenario;

- Goal: Purpose of the scenario;

- Pre-condition: System state before the scenario can start;

- Post-condition: System state after the scenario is performed; 
- Actors: Persons, device or organization structures (active entities) that have a role in the scenario;

- Episodes/Main Flow: Steps to achieve the goal of the scenario;

- Exceptions/Alternative Flows: Exceptions from the course of events;

Other components that might be useful to detail in scenario descriptions are:

Resources (Leite et al., 2000; Sinha et al., 2010) and Context (Leite et al., 2000).

As stated before (Chapter 2), scenarios are usually written in natural language, however, natural language is by definition ambiguous leading to incomplete, inconsistent and incorrect SRS. Ambiguity occurs when two or more users have different interpretations of the same requirement. Incomplete requirements occur because the world is complex; as such, users or clients are not able to identify and develop all relevant requirements. Inconsistent requirements occur when two or more users have conflicting requirements, or the captured requirements are internally inconsistent when one or more requirements override others. Incorrect requirements may occur when the acquired requirements do not accurately reflect the facts, or erroneous predicts about future states.

Numerous techniques have been developed to deal with these quality problems in SRS and each one with its own context of applicability. However, most SRS still do not meet these qualities. According to Glinz (2000), it is not only a problem of applying the right methods and processes for SRS until they yield the desired qualities; the qualities themselves are part of the problem.

So, in order to understand these qualities it is necessary to model the relationships between unambiguity, completeness, consistency and correctness.

\section{2. \\ Modeling Correctness as Non-functional Requirements}

Our proposal is one of the first to represent unambiguity, completeness, consistency and correctness as non-functional requirements based on NFR framework (Chung et al., 2000). Moreover, our contribution also exposes the links and impacts between the properties related to the main NFRs. We introduce a novel perception of correctness and its complex relationships with unambiguity, completeness and consistency describing it as a quality that should be satisficed by contributions of related qualities or properties. 
Based on the literature, we have developed a Soft-goal Interdependency Graph (SIG) for SRS Correctness (Figure 15), which will be the base for cataloged information and will be detailed by a series of decomposition or contribution interdependencies. In order to elaborate the SIG to achieve Correctness, we defined a set of two steps, which are detailed below.

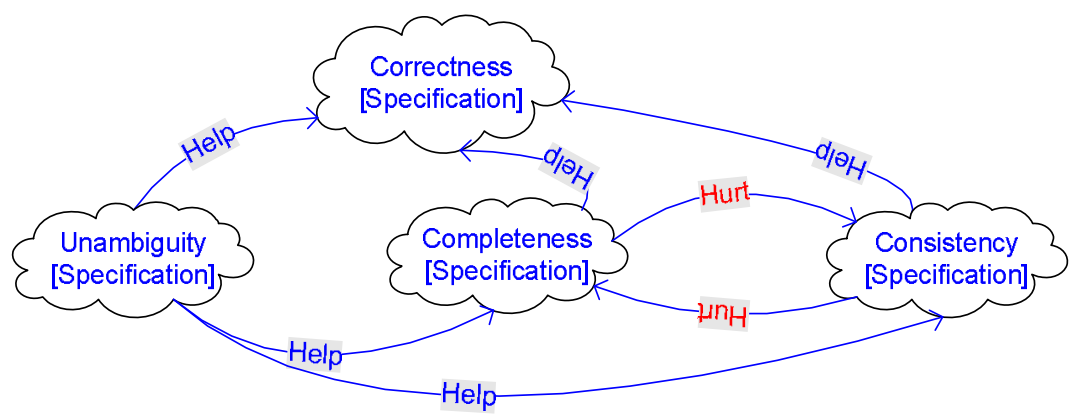

Figure 15 - Initial SIG of SRS Correctness.

\subsection{1.}

\section{Defining the Main NFRs}

In the NFR Approach, unambiguity, completeness, consistency and correctness are non-functional requirements that need to be satisficed (relative satisfaction) by the specifications.

In our work, it is assumed that: (1) correctness is the most important quality; and (2) there is an important causal relationship between unambiguity, completeness, consistency and correctness of SRS. Glinz (2000) uses the term adequacy instead of correctness, and focuses on adequacy as the most important quality.

Zowghi and Gervasi (2003) argue as increasing the completeness of a SRS can decrease its consistency and hence affect the correctness of the final product. Conversely, improving the consistency of the SRS can reduce the completeness, thereby again diminishing correctness. It is frequently the case that in an attempt to maintain consistency within the requirements we remove one or more requirements from the specification and fail to preserve its completeness. Conversely, when we add new requirements to the specification to make it more complete, it is possible to introduce inconsistency in the specification (Zowghi and Gervasi, 2003).

In the context of scenario-based SRS, unambiguity concerns a sentence (scenario consists of many sentences); completeness concerns a single scenario 
and its internal components, and a set of scenarios and their relationships; consistency concerns a set of scenarios and their relationships.

\subsubsection{1.}

\section{Unambiguity}

A specification is unambiguous if and only if, every requirement stated therein has only one interpretation (IEEE, 1998). Although, unambiguity is very difficult to achieve and evaluate because most of the related indicators are subjective; there are defect indicators that can be found. These indicators provide evidence against the existence of unambiguity. These indicators can be grouped and categorized in properties that contribute negatively to Unambiguity: Vagueness, Subjectiveness, Optionality, Weakness, Multiplicity, Implicitly and Quantifiability. Other properties that contribute positively to unambiguity are: Readabiity and Minimality.

Due to inherent ambiguity in natural language-based scenario specifications; NLP techniques can be useful to address several problems that impacts negatively to unambiguity by evaluating linguistic aspects of internal scenario components (Title, Goal, Episodes/Steps, Conditions, Exceptions, and so on). Most of these problems are defects that contribute to unambiguity properties, and they can be identified by performing a lexical or syntactical analysis.

Lexical analysis can be performed to search defect indicators that contribute to Vagueness, Subjectiveness, Optionality, Multiplicity, Quantifiability, Readabiity and Minimality. This analysis is based on the occurrence of special terms (words or phrases) in the sentences or the number of elements in the sentences (Gnesi et al., 2005).

Syntactical analysis is necessary to detect defects related to Weakness and Implicitly properties; however, lexical parsers can be also used to search ambiguity indicators, e.g. weak phrases (Wilson et al., 1998; Tjong, 2008).

Based on results achieved by requirement statements analysis techniques (Wilson et al., 1998; Gnesi et al., 2005; Tjong, 2008), we organized the set of properties that contribute to unambiguity in NL-based scenarios, which can be evaluated by following a checklist with verification heuristics to search defect indicators. The indicators of these properties are collected into dictionaries that contain frequently used words or phrases characterizing defects, and evaluated by 
metrics which can be easily identified and counted by computer programs (See

Table 7).

Table 7 - Properties Related to Unambiguity.

\begin{tabular}{|c|c|c|c|}
\hline Property & Description & Heuristic & Indicator \\
\hline Vagueness & $\begin{array}{l}\text { The sentence contains words } \\
\text { or phrases having a non- } \\
\text { uniquely quantifiable } \\
\text { meaning (Gnesi et al., 2005). }\end{array}$ & $\begin{array}{l}\text { Check that a sentence } \\
\text { does not contain vague } \\
\text { terms }\end{array}$ & $\begin{array}{l}\text { A sentence contains: } \\
\text { adaptability, additionally, } \\
\text { adequate, aggregate, } \\
\text { also, ancillary, arbitrary, } \\
\text {... }\end{array}$ \\
\hline Subjectiveness & $\begin{array}{l}\text { The sentence contains words } \\
\text { or phrases expressing } \\
\text { personal opinions or feeling } \\
\text { (Gnesi et al., 2005). }\end{array}$ & $\begin{array}{l}\text { Check that a } \begin{array}{r}\text { sentence } \\
\text { does not contain } \\
\text { subjective words }\end{array} \\
\end{array}$ & $\begin{array}{l}\text { A sentence contains: } \\
\text { similar, better, similarly, } \\
\text { worse, having in mind, } \\
\text { take into account, take } \\
\text { into consideration, as } \\
\text { possible. }\end{array}$ \\
\hline Optionality & $\begin{array}{l}\text { The sentence contains words } \\
\text { that give the developer } \\
\text { latitude in satisfying the } \\
\text { specification statements that } \\
\text { contain them (Wilson et al., } \\
\text { 1997). }\end{array}$ & $\begin{array}{l}\text { Check that a sentence } \\
\text { does not contain optional } \\
\text { words }\end{array}$ & $\begin{array}{l}\text { A sentence contains: as } \\
\text { desired, at last, either, } \\
\text { eventually, if appropriate, } \\
\text {... }\end{array}$ \\
\hline Weakness & $\begin{array}{l}\text { The sentence contains clauses } \\
\text { that are apt to cause } \\
\text { uncertainty and leave room } \\
\text { for multiple interpretations } \\
\text { (Wilson et al., 1997). }\end{array}$ & $\begin{array}{l}\text { Check that a sentence } \\
\text { does not contain weak } \\
\text { terms }\end{array}$ & $\begin{array}{l}\text { A sentence contains: can, } \\
\text { could, may, might, ought } \\
\text { to, preferred, should, will, } \\
\text { would. }\end{array}$ \\
\hline Multiplicity & $\begin{array}{l}\text { The sentence has more than } \\
\text { one main verb, subject or } \\
\text { object. }\end{array}$ & $\begin{array}{l}\text { Check that a sentence } \\
\text { does not contain } \\
\text { conjunction or disjunction } \\
\text { words }\end{array}$ & $\begin{array}{l}\text { A sentence contains: and, } \\
\text { or, and/or }\end{array}$ \\
\hline Implicitly & $\begin{array}{l}\text { The sentence does not specify } \\
\text { the subject or object by means } \\
\text { of its specific name but uses } \\
\text { pronoun or other indirect } \\
\text { reference (Gnesi et al., 2005). }\end{array}$ & $\begin{array}{l}\text { Check that a sentence } \\
\text { does not contain implicit } \\
\text { words }\end{array}$ & $\begin{array}{l}\text { A sentence contains: } \\
\text { anyone, anybody, } \\
\text { anything, everyone, he, } \\
\text { her, hers, herself, ... }\end{array}$ \\
\hline Quantifiability & $\begin{array}{l}\text { Terms used for quantification } \\
\text { can lead to ambiguity if not } \\
\text { used properly (Arora et al., } \\
\text { 2015). }\end{array}$ & $\begin{array}{l}\text { Check that a sentence } \\
\text { does not contain } \\
\text { quantification words }\end{array}$ & $\begin{array}{l}\text { A sentence contains: all, } \\
\text { any, few, little, many, } \\
\text { much, several, some. }\end{array}$ \\
\hline Readabiity & $\begin{array}{l}\text { It measures how easily an } \\
\text { adult can read and understand } \\
\text { the sentence or Document } \\
\text { (Wilson et al., 1997). }\end{array}$ & $\begin{array}{l}\text { This metric is the } \\
\text { Coleman-Liau Formula } \\
\text { readability metric: } \\
(5.89 * \text { chars/words- } \\
0.3 * \text { sentences/( } 100 * \text { word } \\
\text { s)-15.8]). }\end{array}$ & $\begin{array}{l}\text { The reference value of } \\
\text { this formula for an easy- } \\
\text { to-read technical } \\
\text { document is } 27.60 \text {, if it is } \\
<17.10 \text { and }>55.80 \text { the } \\
\text { document is difficult-to- } \\
\text { read. }\end{array}$ \\
\hline Minimality & $\begin{array}{l}\text { A sentence contains nothing } \\
\text { more than basic attributes } \\
\text { (Lucassen et al., 2015). }\end{array}$ & $\begin{array}{l}\text { Check that a sentence } \\
\text { does not contain } \\
\text { additional information }\end{array}$ & $\begin{array}{l}\text { A sentence contains a Text } \\
\text { after a: dot, hyphen, } \\
\text { semicolon or other } \\
\text { punctuation mark. }\end{array}$ \\
\hline
\end{tabular}




\subsubsection{2.}

\section{Completeness}

A specification is complete if all relevant requirements are present and each requirement is fully developed (Boehm, 1979). Incomplete requirements occur because the world is complex; as such, users or clients are not able to fully understand the impact of present decisions.

Although, completeness is very difficult to define and evaluate because it also depends on external aspects, and violations are hard to detect; there are internal aspects that can be measured. In Leite et al. (2000), the evaluation of completeness in scenario-based specifications is done following a checklist with verification heuristics to detect violations of properties in internal elements of scenarios and their relationships.

Based on Leite et al. (2000), we re-organized the properties that contribute positively to completeness. We understand that a fully developed SRS presents properties related to internal aspects of scenarios (intra-scenario) and their relationships (inter-scenario). The intra-scenario properties include: Atomicity, Simplicity, Uniformity, Usefulness and Conceptually Soundness. The interscenario properties include: Integrity, Coherency and Uniqueness. Other important property related to completeness is Feasibility. For each property, we defined verification heuristics for searching defect indicators that hurt the property.

Table 8, Table 9, Table 10 and Table 11 show the properties that contribute to completeness and heuristics to search common defect indicators that contribute negatively to them. These verification heuristics are driven by syntax checks and by cross-referencing the related scenarios. Some of these heuristics were learned or reported by related work during their experience with scenarios or use cases analysis. 
Table 8 - Intra-scenario Properties Related to Completeness (Continued on Table

9).

\begin{tabular}{|c|c|c|c|}
\hline Property & Description & Heuristic & Indicator \\
\hline \multirow[t]{3}{*}{ Atomicity } & \multirow{3}{*}{ 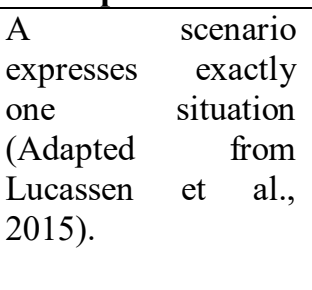 } & $\begin{array}{l}\text { 1. Check that Title defines } \\
\text { exactly one situation; }\end{array}$ & and, or, and/or; \\
\hline & & $\begin{array}{l}\text { 2. Check that Goal satisfies } \\
\text { exactly one purpose; }\end{array}$ & and, or, and/or; \\
\hline & & $\begin{array}{l}\text { 3. Check that Title contains a } \\
\text { verb in infinitive (base) form and } \\
\text { an object; }\end{array}$ & $\begin{array}{l}\text { Missing Action-Verb in Title; } \\
\text { Missing Object in Title; }\end{array}$ \\
\hline \multirow[t]{6}{*}{ Simplicity } & \multirow[t]{6}{*}{$\begin{array}{l}\text { A scenario should } \\
\text { be as readable as } \\
\text { possible; }\end{array}$} & $\begin{array}{l}\text { 1. Check that each } \\
\text { Episode/Exception consists of a } \\
\text { subject, a verb, and optionally, } \\
\text { an object and a prepositional } \\
\text { phrase (It is not a complex } \\
\text { sentence, Ciemniewska and } \\
\text { Jurkiewicz, 2007); }\end{array}$ & $\begin{array}{l}\text { Episode/Exception contains } \\
\text { more than one Action-Verb; } \\
\text { Episode/Exception contains } \\
\text { more than one Subject; } \\
\text { Missing Subject; } \\
\text { Missing Object; }\end{array}$ \\
\hline & & $\begin{array}{l}\text { 2. Check that Episode/Exception } \\
\text { is described from user point of } \\
\text { view, i.e., the present simple } \\
\text { tense and active form of a verb } \\
\text { should be used (Ciemniewska } \\
\text { and Jurkiewicz, 2007); }\end{array}$ & $\begin{array}{l}\text { The Action-verb is not in the } \\
\text { third form; }\end{array}$ \\
\hline & & $\begin{array}{l}\text { 3. Check that Title does not } \\
\text { contain extra unnecessary } \\
\text { information (Adapter from Phalp } \\
\text { et al., 2007). }\end{array}$ & $\begin{array}{l}\text { Title contains text between } \\
\text { brackets (e.g. }(\ldots),\{\ldots\}), \\
\text { URLs, HTML }\end{array}$ \\
\hline & & $\begin{array}{l}\text { 4. Check that Episode } \\
\text { coincidence only takes place in } \\
\text { different situations; }\end{array}$ & $\begin{array}{l}\text { Duplicated Episode Id or } \\
\text { sentence; }\end{array}$ \\
\hline & & $\begin{array}{l}\text { 5. Check that nested IF } \\
\text { statement is not used in a } \\
\text { Conditional Episode, i.e., it can } \\
\text { confuse the user and be difficult } \\
\text { to read (Ciemniewska and } \\
\text { Jurkiewicz, 2007); }\end{array}$ & $\begin{array}{l}\text { More than one Episode inside } \\
\text { a nested } I F \text {; }\end{array}$ \\
\hline & & $\begin{array}{l}\text { 6. Check that Exception is } \\
\text { handed by a simple action, i.e., if } \\
\text { the interruption causes the } \\
\text { execution of a sequence of } \\
\text { sentences, then this sequence } \\
\text { should be extracted to a separate } \\
\text { scenario (Ciemniewska and } \\
\text { Jurkiewicz, 2007); }\end{array}$ & $\begin{array}{l}\text { More than one Sentence inside } \\
\text { a Exception Solution; }\end{array}$ \\
\hline Uniformity & $\begin{array}{lr}\text { Each } & \text { scenario } \\
\text { element } & \text { is } \\
\text { constructed } & \text { using } \\
\text { defined } & \text { scenario } \\
\text { model. } & \end{array}$ & $\begin{array}{l}\text { 1. Check the completeness of } \\
\text { each scenario element (Leite et } \\
\text { al., 2000); }\end{array}$ & $\begin{array}{l}\text { Missing Title } \\
\text { Missing Goal } \\
\text { Missing Actors } \\
\text { Missing Resources } \\
\text { Context does not contain its } \\
\text { relevant sub-components } \\
\text { Missing Episodes } \\
\text { Episode does not contain its } \\
\text { relevant parts (Id, Sentence) } \\
\text { Exception does not contain its } \\
\text { relevant parts (Id, Cause, } \\
\text { Solution) }\end{array}$ \\
\hline
\end{tabular}


Table 9 - Intra-scenario Properties Related to Completeness.

\begin{tabular}{|c|c|c|c|}
\hline Property & Description & Heuristic & Indicator \\
\hline \multirow[t]{6}{*}{ Usefulness } & \multirow{6}{*}{$\begin{array}{l}\text { A scenario does } \\
\text { not contain } \\
\text { superfluous } \\
\text { information, i.e., } \\
\text { there should be } \\
\text { consistency } \\
\text { among scenario } \\
\text { elements. } \\
\text { (Adapted from } \\
\text { Anda et al., 2009). }\end{array}$} & $\begin{array}{l}\text { 1. Check that every Actor } \\
\text { participates in at least one } \\
\text { episode; }\end{array}$ & $\begin{array}{l}\text { Actor does not participate in the } \\
\text { situation; }\end{array}$ \\
\hline & & $\begin{array}{l}\text { 2. Check that every Actor } \\
\text { mentioned in episodes is } \\
\text { included in the Actor element; }\end{array}$ & $\begin{array}{llll}\text { Missing } & \text { Actor in } & \text { Actors } \\
\text { element; } & & & \end{array}$ \\
\hline & & $\begin{array}{l}\text { 3. Check that every Resource is } \\
\text { used in at least one episode; }\end{array}$ & $\begin{array}{l}\text { Resource that is not used in the } \\
\text { situation; }\end{array}$ \\
\hline & & $\begin{array}{l}\text { 4. Check that every Resource } \\
\text { mentioned in episodes is } \\
\text { included in the Resource } \\
\text { element; }\end{array}$ & $\begin{array}{l}\text { Missing Resource in Resources } \\
\text { element; }\end{array}$ \\
\hline & & $\begin{array}{l}\text { 5. Ensure that step numbering } \\
\text { between the main flow and } \\
\text { alternative/exception flow are } \\
\text { consistent (Liu et al., 2015); }\end{array}$ & $\begin{array}{l}\text { Branching Episode of an } \\
\text { exception is missing; }\end{array}$ \\
\hline & & $\begin{array}{l}\text { 6. Check the existence of more } \\
\text { than two and less to } 10 \text { episodes } \\
\text { per scenario (Leite et al., 2000; } \\
\text { Ciemniewska and Jurkiewicz, } \\
\text { 2007); }\end{array}$ & $\begin{array}{l}\text { Number of episodes in each } \\
\text { scenario is less than } 3 \text { or more than } \\
9 ;\end{array}$ \\
\hline \multirow[t]{9}{*}{$\begin{array}{l}\text { Conceptually } \\
\text { Soundness }\end{array}$} & \multirow{9}{*}{$\begin{array}{lr}\text { Internal } & \text { scenario } \\
\text { elements } & \text { are } \\
\text { semantically } & \\
\text { coherent, } & \text { i.e., } \\
\text { scenario elements } \\
\text { satisfy } & \text { the } \\
\text { scenario } & \text { goal } \\
\text { (Leite et al., } \\
2000) . & \end{array}$} & $\begin{array}{l}\text { 1. Check that the Title describes } \\
\text { the Goal; }\end{array}$ & $\begin{array}{l}\text { The corresponding verbs and } \\
\text { objects appearing in the two } \\
\text { compared sentences are not the } \\
\text { same }\end{array}$ \\
\hline & & $\begin{array}{l}\text { 2. Ensure that the set of Episodes } \\
\text { satisfies the Goal and is within } \\
\text { the Context; }\end{array}$ & \multirow[t]{2}{*}{$\begin{array}{l}\text { Difficult to be measured by an } \\
\text { automatic tool; }\end{array}$} \\
\hline & & $\begin{array}{l}\text { 3. Ensure that actions presents in } \\
\text { the Pre-conditions are already } \\
\text { performed; }\end{array}$ & \\
\hline & & $\begin{array}{l}\text { 4. Ensure that Episodes contain } \\
\text { only action to be performed; }\end{array}$ & $\begin{array}{l}\text { Missing Action-Verb in episode } \\
\text { sentences; }\end{array}$ \\
\hline & & $\begin{array}{l}\text { 5. Ensure that Episode condition } \\
\text { contains Linking-Verbs; }\end{array}$ & $\begin{array}{l}\text { Missing Linking-Verb in episode } \\
\text { conditions; }\end{array}$ \\
\hline & & $\begin{array}{l}\text { 6. Ensure that Pre-conditions } \\
\text { contain State-Verbs; }\end{array}$ & $\begin{array}{l}\text { Missing State-Verb in Pre- } \\
\text { conditions; }\end{array}$ \\
\hline & & $\begin{array}{l}\text { 7. Ensure that Post-conditions } \\
\text { contain State-Verbs; }\end{array}$ & $\begin{array}{l}\text { Missing State-Verb in Post- } \\
\text { conditions; }\end{array}$ \\
\hline & & $\begin{array}{l}\text { 8. Ensure that Exception solution } \\
\text { contains only action to be } \\
\text { performed; }\end{array}$ & $\begin{array}{l}\text { Missing Action-Verb in } \\
\text { exception solution; }\end{array}$ \\
\hline & & $\begin{array}{l}\text { 9. Ensure that Exception cause } \\
\text { contains Linking-Verbs or State- } \\
\text { Verbs; }\end{array}$ & $\begin{array}{l}\text { Missing Linking-Verb or State- } \\
\text { Verb in exception causes; }\end{array}$ \\
\hline
\end{tabular}

In Table 10, Integrity and Coherency properties are evaluated checking the main scenario against the related scenarios to it. Uniqueness properties are evaluated checking the main scenario against the other scenarios (related or not). 
Table 10 - Inter-scenario Properties Related to Completeness.

\begin{tabular}{|c|c|c|c|}
\hline Property & Description & Heuristic & Indicator \\
\hline \multirow[t]{3}{*}{ Integrity } & \multirow[t]{3}{*}{$\begin{array}{l}\text { Whenever } \\
\text { scenario includes } \\
\text { an explicit } \\
\text { relationship on } \\
\text { another scenario, } \\
\text { the related scenario } \\
\text { should exist as } \\
\text { another scenario } \\
\text { within the set of } \\
\text { scenarios. }\end{array}$} & $\begin{array}{l}\text { 1. Check that every included } \\
\text { scenario exists within the set of } \\
\text { scenarios (Leite et al., 2000); }\end{array}$ & $\begin{array}{l}\text { Pre-condition identified as } \\
\text { related scenario does not exist } \\
\text { within the set of scenarios; } \\
\text { Post-condition identified as } \\
\text { related scenario does not exist } \\
\text { within the set of scenarios; } \\
\text { Episode sentence identified as } \\
\text { related scenario does not exist } \\
\text { within the set of scenarios; } \\
\text { Exception solution identified } \\
\text { as related scenario does not } \\
\text { exist within the set of } \\
\text { scenarios; } \\
\text { Constraint identified as related } \\
\text { scenario does not exist within } \\
\text { the set of scenarios; }\end{array}$ \\
\hline & & $\begin{array}{l}\text { 2. Check that every Exception is } \\
\text { treated by a scenario (Leite et al., } \\
2000 \text { ); }\end{array}$ & $\begin{array}{l}\text { Complex Exception Solution } \\
\text { must be treated by a scenario; }\end{array}$ \\
\hline & & $\begin{array}{l}\text { 3. Check that a Pre-condition (not } \\
\text { described as another scenario) of a } \\
\text { scenario is satisfied by a Post- } \\
\text { condition of other scenario, i.e., it } \\
\text { is possible to infer relationships } \\
\text { from pre-condition/post-condition } \\
\text { (Leite et al., 2000); }\end{array}$ & $\begin{array}{l}\text { Missing pre-condition/post- } \\
\text { condition; }\end{array}$ \\
\hline \multirow[t]{3}{*}{ Coherency } & \multirow{3}{*}{$\begin{array}{l}\text { Internal elements } \\
\text { of explicitly related } \\
\text { scenarios should be } \\
\text { precise and use a } \\
\text { common } \\
\text { terminology, e.g. } \\
\text { pre-conditions of } \\
\text { sub-scenarios are } \\
\text { coherent with main } \\
\text { scenario } \\
\text { conditions. }\end{array}$} & $\begin{array}{l}\text { 1. Check coherence between the } \\
\text { related scenario Pre-conditions and } \\
\text { the main scenario Pre-conditions; }\end{array}$ & $\begin{array}{l}\text { Difficult to be measured by an } \\
\text { automatic tool; }\end{array}$ \\
\hline & & $\begin{array}{l}\text { 2. Check that Geographical and } \\
\text { Temporal location of the related } \\
\text { scenarios are equal or more } \\
\text { restricted than those of the main } \\
\text { scenario (Leite et al., 2000); }\end{array}$ & $\begin{array}{l}\text { Related scenario Geographical } \\
\text { location is not in the set of } \\
\text { Geographical locations of root } \\
\text { scenario; } \\
\text { Related scenario Temporal } \\
\text { location is not in the set of } \\
\text { Temporal locations of root } \\
\text { scenario; }\end{array}$ \\
\hline & & $\begin{array}{l}\text { 3. Check that every referenced } \\
\text { scenario does not reference the } \\
\text { main scenario (Adapted from } \\
\text { Sinnig et al., 2009); }\end{array}$ & $\begin{array}{l}\text { Circular inclusion between } \\
\text { two scenarios; }\end{array}$ \\
\hline \multirow[t]{5}{*}{ Uniqueness } & \multirow{5}{*}{$\begin{array}{l}\text { A scenario is } \\
\text { unique when no } \\
\text { other scenario is } \\
\text { the same or too } \\
\text { similar, } \\
\text { duplicates are } \\
\text { avoided because } \\
\text { they are source of } \\
\text { inconsistencies } \\
\text { (Adapted from } \\
\text { Lucassen et al., } \\
\text { 2015); }\end{array}$} & $\begin{array}{l}\text { 1. Check that the Title of a } \\
\text { scenario is not already included in } \\
\text { another scenario; }\end{array}$ & $\begin{array}{l}\text { Title coincidence between two } \\
\text { scenarios; }\end{array}$ \\
\hline & & $\begin{array}{l}\text { 2. Check that the Goal of a } \\
\text { scenario is not already included in } \\
\text { another scenario; }\end{array}$ & $\begin{array}{l}\text { Goal coincidence between two } \\
\text { scenarios; }\end{array}$ \\
\hline & & $\begin{array}{l}\text { 3. Check that the Context Pre- } \\
\text { condition of a scenario is not } \\
\text { already included in another } \\
\text { scenario; }\end{array}$ & $\begin{array}{l}\text { Pre-condition coincidence } \\
\text { between two scenarios; }\end{array}$ \\
\hline & & $\begin{array}{l}\text { 4. Check that the set of Episodes of } \\
\text { a scenario is not already included } \\
\text { in another scenario; }\end{array}$ & $\begin{array}{l}\text { Episodes coincidence between } \\
\text { two scenarios; }\end{array}$ \\
\hline & & $\begin{array}{l}\text { 5. Check the similarity of the } \\
\text { scenario with other scenarios using } \\
\text { syntactic analysis; }\end{array}$ & $\begin{array}{l}\text { Titles share the same Action- } \\
\text { Verb and the direct Object; }\end{array}$ \\
\hline
\end{tabular}


Table 11 - Feasibility Property Related to Completeness.

\begin{tabular}{|c|c|c|c|}
\hline Property & Description & Heuristic & Indicator \\
\hline \multirow[t]{2}{*}{ Feasibility } & \multirow{2}{*}{$\begin{array}{l}\text { It is possible to } \\
\text { perform each } \\
\text { operation described } \\
\text { in a scenario and } \\
\text { each } \\
\text { internal/external } \\
\text { condition is not } \\
\text { violated. }\end{array}$} & $\begin{array}{l}\text { 1. Check that is possible to } \\
\text { derive an initial system } \\
\text { design from the current } \\
\text { scenario (Adapted from } \\
\text { Denger et al., 2005); }\end{array}$ & $\begin{array}{l}\text { There are } r \text { not } \\
\text { relationships } \\
\text { scenarios; }\end{array}$ \\
\hline & & $\begin{array}{l}\text { 2. Check that initial system } \\
\text { design does not contain } \\
\text { isolated sub-systems; }\end{array}$ & Unreachable operations; \\
\hline
\end{tabular}

\subsubsection{3.}

\section{Consistency}

A specification is consistent when two or more requirements are not in conflict with one another or with governing specifications and objectives (Boehm, 1979). Inconsistent requirements occur when two or more users have conflicting requirements, or the captured requirements are internally inconsistent when one or more requirements override others.

Evaluation of consistency with respect to external specifications is very difficult to perform. Consistency defects are difficult to detect or only with mucheffort. However, internal aspects of consistency can be evaluated when the behavior of a set of scenarios is simulated, and defects are identified in scenario relationships.

One of the main strategies to ensure the consistency is the evaluation of dynamic aspects of a SRS. This is done by first mapping scenario representations into executable models, and performing a rigorous behavioral analysis to detect violations (defects) of properties that contribute positively to consistency. There exist several tools to perform rigorous analysis on executable models (e.g. PetriNet). These tools generate a reachability graph which contains the different states of execution, and traverse this graph for searching defect indicators.

Table 12 shows the properties that contribute to consistency and verification heuristics for searching defect indicators that hurt these properties. Dynamic properties that influence the consistency are: Non-interferential, Boundedness, Reversibility and Liveness. 
Table 12 -Properties Related to Consistency.

\begin{tabular}{|c|c|c|c|}
\hline Property & Description & Heuristic & Indicator \\
\hline $\begin{array}{l}\text { Non- } \\
\text { interferential }\end{array}$ & 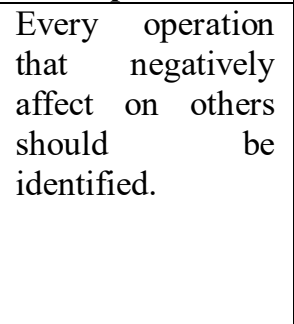 & $\begin{array}{l}\text { Check Non-determinism: A non- } \\
\text { deterministic behavior occurs when a } \\
\text { set of operations are simultaneously } \\
\text { enabled. If the reachability graph } \\
\text { reveals non-deterministic execution } \\
\text { paths, a warning is reported to } \\
\text { indicate wrong information (Lee et } \\
\text { al., 1998; Lee et al., 2001). }\end{array}$ & $\begin{array}{l}\text { Simultaneously } \\
\text { enabled } \\
\text { operations; }\end{array}$ \\
\hline Boundedness & $\begin{array}{l}\text { This property } \\
\text { refers to the } \\
\text { limited capacity } \\
\text { of a a } \\
\text { communication } \\
\text { channel or shared } \\
\text { resource. }\end{array}$ & $\begin{array}{l}\text { Check Overflow: An executable } \\
\text { model is overflowed when the } \\
\text { number of elements in some } \\
\text { communication channel or resource } \\
\text { exceeds a finite capacity (Zhao and } \\
\text { Duan, 2009). }\end{array}$ & $\begin{array}{l}\text { Overflowed } \\
\text { resource; }\end{array}$ \\
\hline Reversibility & $\begin{array}{l}\text { The behavior } \\
\text { should reach its } \\
\text { initial state again. }\end{array}$ & $\begin{array}{l}\text { Check Reversibility: Reversibility of } \\
\text { an executable model guarantees that } \\
\text { the described behavior reaches its } \\
\text { initial state again. If the executable } \\
\text { model is not reversible, the automatic } \\
\text { error recovery is not possible } \\
\text { (Cheung et al., 2006). }\end{array}$ & $\begin{array}{l}\text { There are no a } \\
\text { path from an } \\
\text { operation to the } \\
\text { initial state; }\end{array}$ \\
\hline Liveness & $\begin{array}{l}\text { Every operation } \\
\text { can be executed in } \\
\text { the future. }\end{array}$ & $\begin{array}{l}\text { Check Liveness: Liveness is closely } \\
\text { related to the complete absence of } \\
\text { deadlocks. An executable model is } \\
\text { deadlocked if no process can make } \\
\text { any progress, generally because each } \\
\text { is waiting for communication with } \\
\text { others (Lee et al., 1998). }\end{array}$ & $\begin{array}{l}\text { Path to deadlock; } \\
\text { Never enabled } \\
\text { operations; }\end{array}$ \\
\hline
\end{tabular}

\subsubsection{4. \\ Correctness}

A specification is correct if, and only if, every requirement stated therein is one that the software shall meet (IEEE, 1998). Incorrect requirements may occur when the acquired requirements do not accurately reflect the facts, or erroneous predicts about future states.

Correctness is the main requirements quality, and it is difficult to evaluate and achieve. Therefore, having an unambiguous, complete and consistent set of scenarios contributes positively to more correct SRS.

\subsection{2. Modeling the SIG}

In order to evaluate unambiguity, completeness, consistency and consequently correctness, we apply the NFR qualitative reasoning approach (Chung et al., 2000); the goal here is to achieve good correctness in scenariobased SRS. 
Figure 16 illustrates the SIG that models the SRS Correctness and how SRS Unambiguity, Completeness and Consistency impact positively (help) to Correctness. We assume that a SRS is more correct, if it is perceived as unambiguous, complete and consistent with respect to real user's needs. Interdependency between SRS Consistency and Completeness impacts negatively (hurt) on both (Zowghi and Gervasi, 2003).

Properties that contribute negatively to Unambiguity are modeled using HURT links - in Vagueness, Subjectiveness, Optionality, Weakness, Multiplicity, Implicitly and Quantifiability. Also, properties that contribute positively to Unambiguity are modeled using HELP links - in Readabiity and Minimality softgoals.

Completeness and Consistency are decomposed - using AND links - in Internal and External soft-goals, following the lead of (Boehm, 1979) and (Zowghi and Gervasi, 2003). Evaluating External Completeness and External Consistency is a hard problem because it depends on external specifications, external domain models and user's needs satisfaction.

Properties that contribute positively to Internal Completeness are modeled using HELP links - in Atomicity, Simplicity, Uniformity, Usefulness, Conceptually Soundness, Integrity, Coherency, Uniqueness and Feasibility soft-goals. These soft-goals can be operationalized by: (1) Writing Scenarios using Regular Languages (Hsia et al., 1994; Cheung et al., 2006), OR (2) Writing Scenarios following concrete syntax rules (Leite et al., 2000; Anda and Sjoberg, 2002; Denger et al., 2005; Phalp et al., 2007; Sinha et al., 2010), OR (3) Analyzing scenarios using NLP techniques.

Properties that contribute positively to Internal Consistency are modeled using HELP links - in Non-interferential, Boundedness, Reversibility and Liveness soft-goals. These soft-goals can be operationalized by: (1) Writing scenarios using Regular Languages (Hsia et al., 1994; Cheung et al., 2006), OR (2) Analysis of Scenarios with Petri-Nets (Lee et al., 1998; Lee et al., 2001; Zhao and Duan, 2009; Somé, 2010); OR (3) OR Analysis of Scenarios with LTS (Sinnig et al., 2009). 


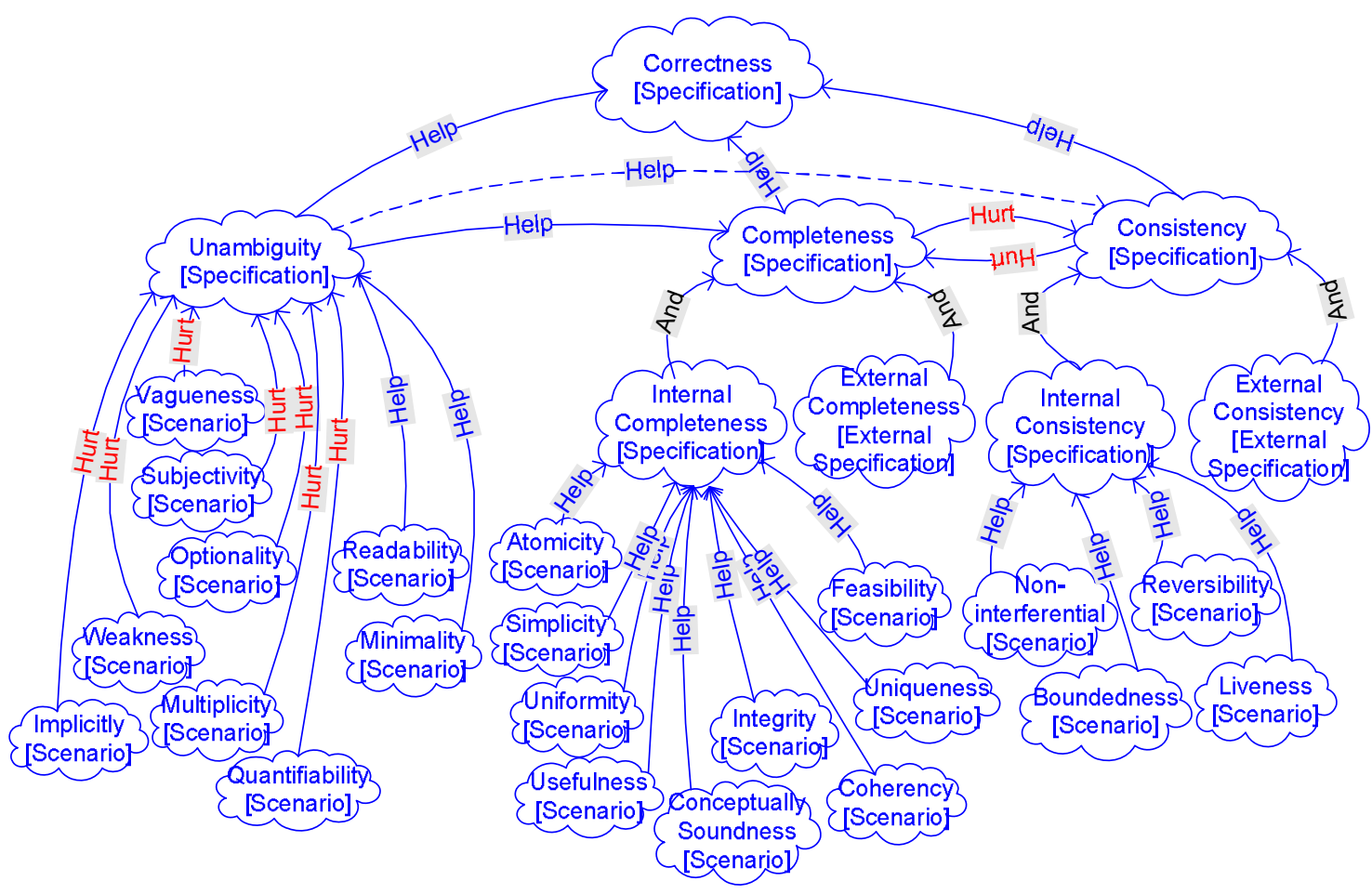

Figure 16 - SIG of SRS Correctness.

\section{3.}

\section{Final Considerations}

The process of scenario-based SRS verification is a complicated activity; no single solution is effective to resolve the challenges of dealing with Unambiguity, Completeness, Consistency and Correctness. And, there is a lack of systematic approaches to model and organize the related properties to Unambiguity, Completeness, Consistency and Correctness of scenario-based SRS.

We have used the NFR framework to help the organization (Quality Model for Scenarios) of properties that contribute to Unambiguity, Completeness, Consistency and Correctness qualities with operationalizations using NLP techniques, Petri-Nets or LTSs, which can enable the automated SRS verification.

The quality model for scenarios can be used to evaluate static and dynamic properties of scenario-based SRS; and, it provides benefits due to the following reasons: (1) it identifies properties to be evaluated due to individual and interacting scenarios; (2) it shows what kind of operationalizations can be made to support the evaluation of properties; and (3) it is possible to reuse the patterns and specialize them for more specific scenario languages. 


\section{4 \\ Scenario Analysis Approach}

Scenarios are the main technique for modeling user requirements, which have been widely adopted by user-oriented approaches for software development. Due to natural language, defects are inevitably introduced in scenario descriptions. In this chapter, we discuss our approach for detecting defects in acquired scenario descriptions. It detects wrong information, missing information and erroneous situations that can be hidden within scenarios and their relationships with related scenarios.

For it, we (1) describe scenarios using a Restricted-from of Natural Language (RNL) Scenario technique, which presents a concrete grammar based on linguistic patterns to write sentences and describes the relationships among scenarios, and heuristics to identify non-explicit relationships; (2) instantiate the Quality Model for Scenarios (Chapter 3); and (3) consider the results achieved by NLP and Petri-Net based related work.

In our scenario analysis approach: First, requirements engineers start to describe the different functionalities, services or situations of the system as textual scenarios. Second, irrelevant information within scenario elements are removed. Third, by an automatic transformation, an initial system design is derived by translating these scenarios into Place/Transition Petri-Nets, and synthesizing them into a consistent whole Petri-Net which represents the relationships among related scenarios. Fourth, from these representations (Scenarios and their resulting Petri-Nets), defects that hurt unambiguity and completeness of scenarios are detected by analyzing structural properties of scenarios, and defects that hurt consistency and correctness of scenarios are detected by analyzing behavioral properties of equivalent Petri-Nets. Fifth, the analysis outcome is formatted and returned to the requirements engineers. Sixth, if defects are found, the analysis feedback is used to improve the scenario descriptions, since the identified defects and their causes can be traced to the scenarios. The approach also shows the source of errors detected in equivalent 
Petri-Nets, i.e., Petri-Net analysis errors are traced into defects in scenarios or their relationships. With the feedback provided by the approach, the requirements engineer can improve the scenario descriptions and then the process starts again in pre-process activity until no defects are detected.

Below in Figure 17, we detail the activities of our analysis approach using the SADT language (Ross, 1977). The activities two to five (Pre-process, Derive, Analyze and Generate) are performed automatically by the C\&L (2015) tool.

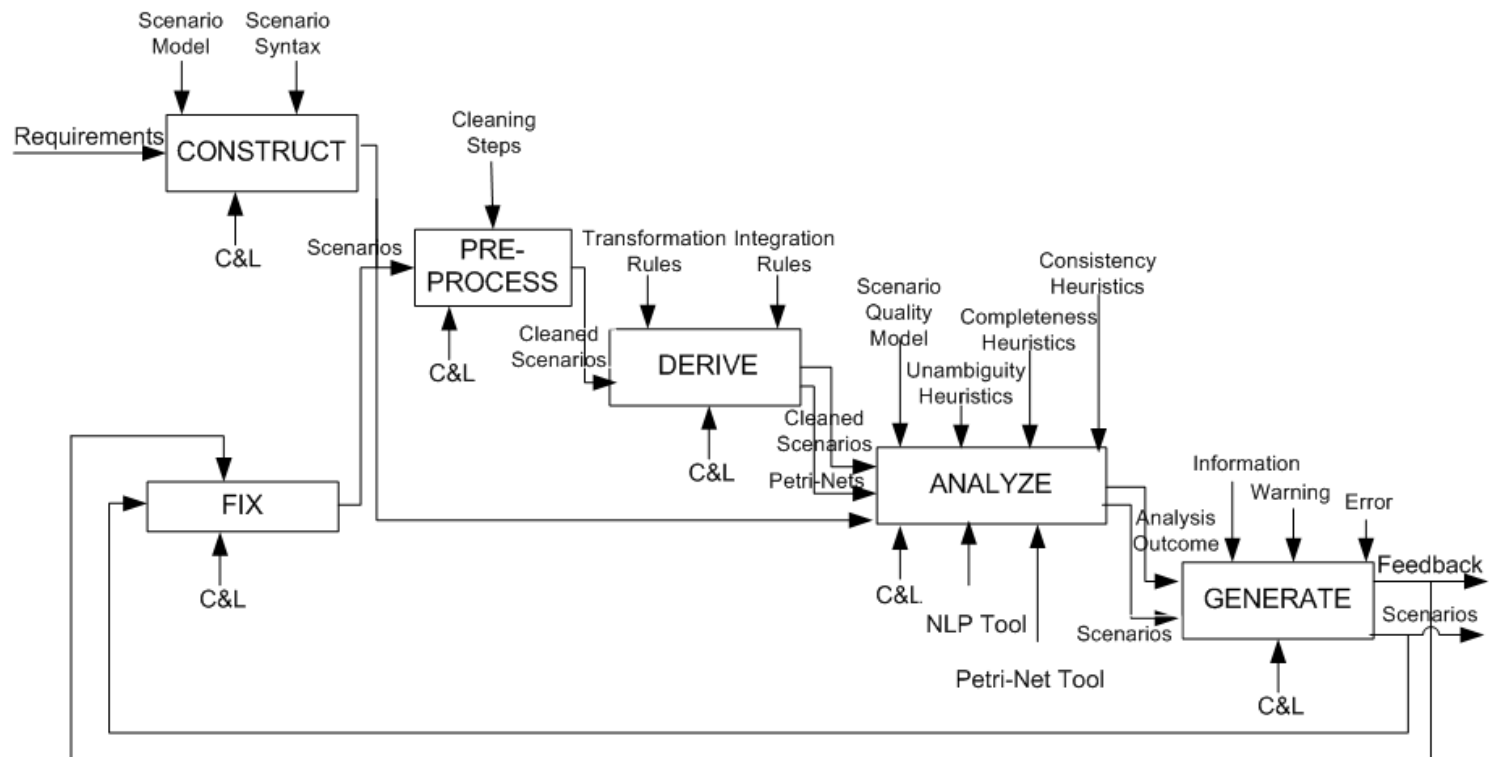

Figure 17- SADT of the Scenarios Analysis Approach.

\section{1.}

\section{Writing Restricted-form of Natural Language Scenarios}

This activity is carried out by requirements engineers, which start to elicit the requirements and describe the different situations, functionalities, services or tasks of the system as scenario representations. In Leite et al. (2000), the scenario construction process is detailed and decomposed in other activities.

As mentioned before, the language used to write these scenarios is a Restricted-form of Natural Language (RNL). Using RNL it is possible to write imperative and declarative sentences. An imperative sentence describes actor events; and a declarative sentence describes actor or resource states. Thus, software requirements specifications can be described as clear and well-defined scenario descriptions.

The use of RNL restricts the vocabulary used to write scenarios and prevents the introduction of ambiguous sentences in the scenario specification, contributing to the quality of documentation. RNL is also necessary to define 
syntax rules for sentences construction. Moreover, it helps the automatic transformation of textual scenarios into formal executable models.

The natural language based-scenario used in this work is an adaptation of a previous language (Leite et al., 2000). Unlike Leite et al. (2000), our focus is the analysis of scenarios. For this purpose, a new scenario language is defined by adding the pre-condition and post-condition attributes, and the repetition structure control to the grammar proposed in (Leite et al., 2000).

This sub-section begins with a definition of an abstract conceptual model for the proposed scenario language. Next, it presents a concrete grammar based on linguistic patterns to write sentences within scenarios using a restricted-form of natural language. It also describes the relationships among scenarios, and heuristics to identify non-explicit relationships.

\subsection{1. Scenario}

Scenario specifications capture system behaviors or situations in the domain (Leite et al., 2000) and, it helps the understanding of the requirements by the developers and other stakeholders.

In literature, the term scenario is used with different meanings in different contexts, and there is no clear distinction between scenarios and use cases. While some authors consider that each scenario corresponds to one use case (Glinz, 2000), others define a scenario as sequences of use case steps that represent different paths through a use case (Cockburn, 2001). According to Glinz (2000), a scenario may comprise a concrete sequence of interaction steps (instance scenario) or a set of possible interaction steps (type scenario). Based on definitions from Glinz (2000) and Leite et al. (2000), we stated a scenario definition that enables a further transformation.

A scenario is a collection of partially ordered event occurrences, each guarded by a set of conditions (pre-condition and post-condition) or restricted by constraints. An event is an operation or an interaction involving persons, organizations, system, environment, or system's components. A condition is an actor/resource/system state (e.g. the availability of some resource). An actor can be a user, organization, device, the system, system's components or agents in the environment; they have a role in the scenario or act on the Universe of Discourse. 
Figure 18 presents an abstract conceptual model for the proposed scenario language, using a class diagram. According to our conceptual model, the scenario language is composed of the main entity Scenario, and the Context, Resource, Actor, Episode, Exception and Constraint entities.

In the proposed language, a scenario starts in an initial state (context) with all necessary Resources and Actors, and must satisfy a Goal that is reached by performing its Episodes. The episodes describe the operational behavior of the situation, which includes the main course of action and possible alternatives. An Exception can arise during the execution of episodes, and indicates that there exists an obstacle to satisfy the goal. The treatment to this exception does not need to satisfy the scenario goal. A scenario pre-condition, post-condition, constraint, episode or exception can be expressed by another scenario.

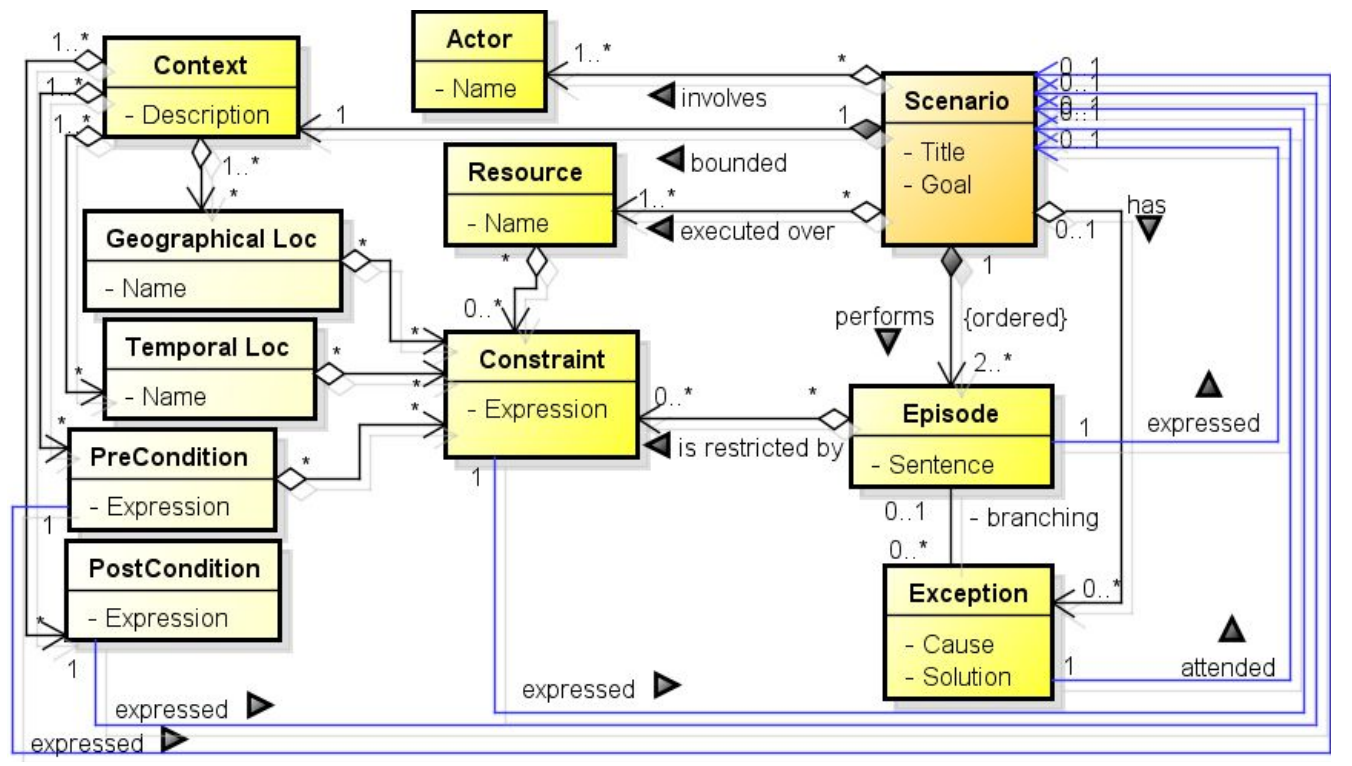

Figure 18 - Scenario Conceptual Model.

\subsubsection{1.}

Title

The title of a scenario identifies the scenario using a declarative sentence, and it must be unique.

\subsubsection{2.}

Goal

The goal describes the purpose of the scenario using a declarative sentence, and it gives a general idea about the scenario main purpose and how it is achieved. 


\subsubsection{3.}

Context

The context describes the scenario initial state using declarative sentences, and it must be described through at least one of the following sub-components: Pre-condition, Post-condition, Geographical or Temporal location.

Pre-condition expresses the initial state of the scenario. Post-condition expresses the final state of the scenario. Geographical location represents the physical set of the scenario. Temporal location is the time specification for the scenario development.

Pre-conditions, Post-conditions, Geographical locations and Temporal locations may be expressed by one or more simple sentences linked by the logical connectors $\boldsymbol{A N D}$ or $\boldsymbol{O R}$. A pre-condition or post-condition sentence can be detailed in another scenario.

\subsubsection{4. Resources}

Resources are an enumeration of relevant physical elements or information (passive entities) that must be available in the scenario. They are used by the actors in the episodes to achieve scenario's goal. Resources must appear in at least one of the episodes.

\subsubsection{5.}

Actors

Actors are an enumeration of persons, device or organization structures (active entities) that have a role in the scenario. They are directly involved with a situation. Actors must appear in at least one of the episodes.

\subsubsection{6. Episodes}

They are a set of actions that give an operational description of behavior. They represent the main flow, which is a sequence of steps where everything works as expected. An episode can be described as a scenario.

An episode $e$ is a 7-tuple (Id, Sentence, Type, Condition, Constraint, Precondition, Post-condition). Every episode is identified through an identifier Id and a Type. An episode performs an action - Sentence (imperative) that can use (or modify) resources and be executed by actors. Depending on the episode type, 
conditions are added (conditional or loop episode). Optionally, the following attributes can be added: (1) Constraints that restrict the quality with witch the episode is performed; (2) Pre-conditions that we expect are already satisfied before the episode is performed; (3) Post-conditions that we expect will be achieved after the episode occurs. An episode is carried out only when the set of pre-conditions are satisfied.

Pre-condition and post-condition are described as declarative sentences involving relevant actor/resource/system states (e.g. the availability of a resource). They are different of context's pre-condition and context's post-condition because: (1) context's pre-conditions are the state of the system before the scenario is started; (2) context's post-conditions are the state of the system when the set of episodes are carried out. These attributes are important in the modeling, analysis and design synthesis of concurrent systems (Lee et al., 1998; Cheung et al., 2006). Pre-conditions and post-conditions can be expressed by one or more single sentences linked by the logical connectors AND or OR.

Episodes are simple, conditional, optional and loop ones. Simple episodes are those necessary to complete the scenario. Conditional episodes are those whose occurrence depends on internal or external conditions. Optional episodes are those that may or may not take place depending on conditions that cannot be detailed. Loop episodes can be used as repetition structures whose occurrence depends on internal or external conditions. Internal conditions may be due to alternative pre-conditions, actors or resources constraints and previous episodes. External conditions may be provided by external actors or another scenario. Conditions can be expressed by one or more single logical sentences linked by the logical connectors AND or OR.

Independently of its type, an episode can be expressed as a single action or can itself be conceived as a scenario, thus enabling the possibility of decomposition of a scenario in sub-scenarios.

A sequence of episodes implies a precedence order, but a non-sequential order can be bounded by the symbol "\#” allowing the grouping of two or more episodes. This is used to describe indistinct sequential order, concurrent or parallel episodes (\#<Episode Series $>\#)$. 


\subsubsection{7. Exception}

Exceptions are situations that prevent the proper course of the scenario. The treatment of the exception may be expressed through a sentence or detailed in another scenario. An exception hinders the achievement of the scenario goal, and it can describe an alternative or exceptional execution flow.

An exception ex is a 4-tuple (Id, Cause, Solution, Post-condition). Every exception is identified through an identifier Id. An exception: (1) is caused by invalid input data or the lack or malfunction of a necessary resource (resource/system state) - Cause; (2) is treated by an imperative sentence Solution; and optionally (3) may generate effects on the resource/system states, or simply produce a message - Post-condition .

An exception is always branched from an episode of the main execution flow. Causes and post-conditions can be expressed by one or more single sentences linked by the logical connectors AND or OR.

\subsubsection{8. Constraint}

Scope or non-functional requirement referring to a given entity, and described as a declarative sentence that restricts the quality with witch: (1) the goal is achieved, (2) a resource is needed and (3) an episode is performed. Thus, Constraint is an attribute of resource, episode or context's sub-components. Constraints can be expressed by a set containing one or more single sentences.

Figure 19 presents a scenario example. This example itself is explained later. The "Submit Order" scenario describes the interactions between the Online Broker System and its partner services, Local Supplier and International Supplier. 


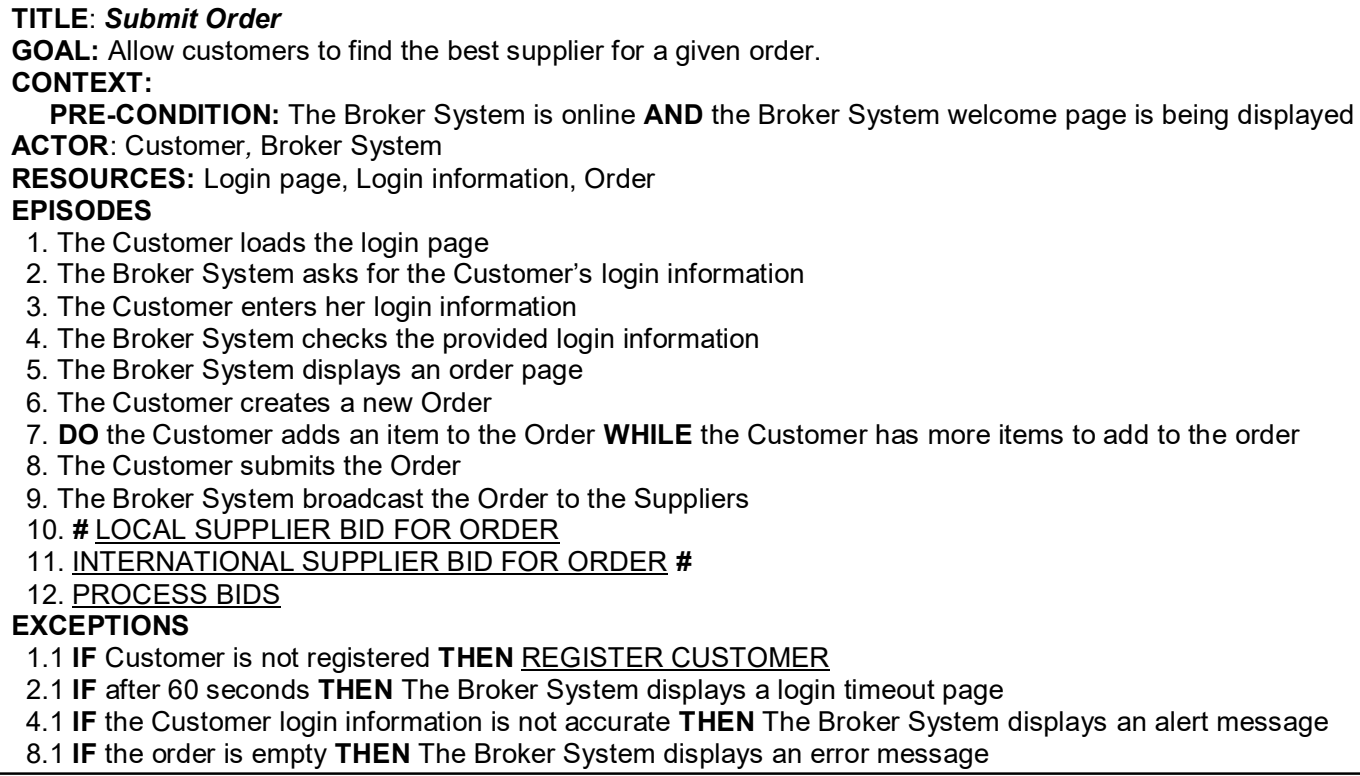

Figure 19 - Example of scenario (Submit Order) in the Online Broker System.

\subsection{2.}

\section{Restricted-form of Natural Language}

As already mentioned, scenario elements (Title, Goal, Context, Resource, Actor, Episodes and Exception) are written using a Restricted-form of Natural Language (RNL). In order to reduce ambiguity in natural language-based sentences, we have defined a scenario grammar for writing sentences in accordance to its conceptual model (Figure 18). Using this grammar, it is possible to write imperative (title, episode sentence or exception) and declarative (conditions or states) sentences. An imperative sentence describes actor events; and a declarative sentence describes actor or resource states.

A sentence in scenario grammar is basically defined according to the format "Subject + Verb + Predicate", where subject, verb and predicate represent the subject, main verb and objects affected by the main verb, respectively. Therefore, the sentence construction is centered on the main verb.

Table 13 shows the grammar for writing scenario elements using partial Extended-BNF (ISO/IEC 14977, 2015). The scenario model should be seen as a syntax and structural guidelines to: (1) obtain a homologous description style, (2) demonstrate the aspects that scenarios can cover and (3) facilitate the automated analysis (Leite et al., 2000).

According to the grammar described in Table 13, a Scenario must be described by: Title, Goal, Context, Resource, Actor, Episodes and Exception. 
Table 13 - Scenario Grammar

\begin{tabular}{|c|c|}
\hline TYPE & DESCRIPTION \\
\hline$<$ Scenario $>$ & $\begin{array}{l}\text { TITLE: }<\text { Title }>+ \\
\text { GOAL: }<\text { Goal }>+ \\
\text { CONTEXT: }<\text { Context }>+ \\
\text { RESOURCE: }\{<\text { Resource }>\}_{1}{ }^{\mathrm{N}}+ \\
\text { ACTOR: }\{<\text { Actor }>\}_{1}{ }^{N}+ \\
\text { EPISODES: }<\text { Episodes }>+ \\
\text { EXCEPTION: }\{<\text { Exception }>\}\end{array}$ \\
\hline$\langle$ Title $>$ & $([$ Actor $\mid$ Resource $]+$ Action-Verb + Predicate $) \mid$ Phrase \\
\hline$<$ Goal $>$ & [Actor | Resource] + Verb + Predicate \\
\hline$<$ Context $>$ & $\begin{array}{l}{[\text { [GEOGRAPHICAL LOCATION: }<\text { Geographical Location }>]+} \\
{[\text { TEMPORAL LOCATION: }<\text { Temporal Location }>]+} \\
{[\text { PRE-CONDITION }:<\text { Pre-condition }>]+} \\
{[\text { POST-CONDITION }:<\text { Post-condition }>]}\end{array}$ \\
\hline $\begin{array}{l}\text { <Geographical } \\
\text { Location }>\end{array}$ & $\begin{array}{l}\text { Name }+[\text { CONSTRAINT: }\{<\text { Constraint }>\}] \mid \\
<\text { Geographical Location }><\text { connective }><\text { Geographical Location }>\end{array}$ \\
\hline <Temporal Location> & $\begin{array}{l}\text { Name }+[\text { CONSTRAINT: }\{<\text { Constraint }>\}] \mid \\
<\text { Temporal Location }><\text { connective }><\text { Temporal Location }> \\
\end{array}$ \\
\hline$<$ Pre-condition $>$ & $\mid<$ expression $>\mid<$ Title $>\mid<$ Pre-condition $><$ connective $><$ Pre-condition $>$ \\
\hline$<$ Post-condition $>$ & $\mid<$ expression $>\mid<$ Title $>\mid<$ Post-condition $><$ connective $><$ Post-condition $>$ \\
\hline <expression $>$ & $(($ Actor $\mid$ Resource $)+$ State-Verb + Predicate $) \mid$ Phrase \\
\hline <connective> & AND $\mid$ OR \\
\hline$<$ Resource $>$ & Name $+[$ CONSTRAINT: $\{<$ Constraint $>\}]$ \\
\hline$\langle$ Actor $>$ & Name \\
\hline$<$ Episodes $>$ & $\mid<$ Group $>\mid<$ Episodes $><$ Group $>$ \\
\hline <Group $>$ & $\mid<$ Sequential Group $>\mid<$ Non-Sequential Group $>$ \\
\hline <Sequential Group > & $\mid<$ Episode $><$ Episode $>\mid<$ Sequential Group $><$ Episode $>$ \\
\hline <Non-Sequential Group > & $\{<$ Episode $>\} \#<$ Episode Series $>\#\{<$ Episode $>\}$ \\
\hline$\langle$ Episode Series $>$ & $\mid<$ Episode $><$ Episode $>\mid<$ Episode Series $><$ Episode $>$ \\
\hline <Episode $>$ & $\begin{array}{l}\text { <Simple Episode }>\mid<\text { Conditional Episode }>\mid<\text { Optional Episode }>\mid \\
\text { <Loop Episode }>\end{array}$ \\
\hline <Simple Episode $>$ & $\begin{array}{l}<\text { Id }><\text { Episode Sentence }>+[\text { PRE-CONDITION: }<\text { Pre-condition }>]+ \\
{[\text { POST-CONDITION: }<\text { Post-condition }>]+[\text { CONSTRAINT: }\{<\text { Constraint }>\}]}\end{array}$ \\
\hline$<$ Conditional Episode $>$ & $\begin{array}{l}<\mathrm{Id}>\text { IF }<\text { Condition }>\text { THEN }<\text { Episode Sentence }>+ \\
{[\text { PRE-CONDITION }:<\text { Pre-condition }>]+[\text { POST-CONDITION }:<\text { Post-condition }>]} \\
+[\text { CONSTRAINT: }\{<\text { Constraint }>\}]\end{array}$ \\
\hline$<$ Optional Episode $>$ & $\begin{array}{l}<\text { Id }>\text { "[" < Episode Sentence }>\text { "]" + [PRE-CONDITION: }<\text { Pre-condition>] + } \\
{[\text { POST-CONDITION: }<\text { Post-condition }>]+[\text { CONSTRAINT: }\{<\text { Constraint }>\}]}\end{array}$ \\
\hline <Loop Episode $>$ & $\begin{array}{l}<\mathrm{Id}>\text { DO }<\text { Episode Sentence }>\text { WHILE }<\text { Condition }>+ \\
{[\text { PRE-CONDITION: }<\text { Pre-condition }>]+[\text { POST-CONDITION }:<\text { Post-condition }>]} \\
+[\text { CONSTRAINT: }\{<\text { Constraint }>\}]\end{array}$ \\
\hline$\langle\mathbf{I d}\rangle$ & <id-chair $>\{(.|,| ; \mid:)+<$ id-char $>\}+[.|,| ; \mid:]$ \\
\hline <id-char> & Letter $\mid$ Digit \\
\hline <Episode Sentence $>$ & $([$ Actor $\mid$ Resource $]+$ Action-Verb $+[$ Direct-Object-Predicate $]) \mid<$ Title $>$ \\
\hline$<$ Condition> & $\mid<$ atomic sentence $>\mid<$ Condition $><$ connective $><$ Condition $>$ \\
\hline$<$ atomic sentence $>$ & $(($ Actor $\mid$ Resource $)+$ Linking-Verb + Predicate $) \mid$ Phrase \\
\hline <Exception> & $<$ Id $>$ IF $<$ Cause $>$ THEN $<$ Solution $>+[$ POST-CONDITION: $<$ Post-condition $>$ ] \\
\hline$<$ Cause $>$ & $\mid<$ atomic sentence $>\mid<$ expression $>\mid<$ Cause $><$ connective $><$ Cause $>$ \\
\hline$<$ Solution> & $([$ Actor $\mid$ Resource $]+$ Action-Verb + [Direct-Object-Predicate $]) \mid<$ Title $>$ \\
\hline$<$ Constraint $>$ & $\mid([$ Actor $\mid$ Resource $]+[$ MUST $]+[$ NOT $]+$ Predicate $) \mid<$ Title $>\mid$ Phrase \\
\hline
\end{tabular}

In Table 13, + means composition, $\{\mathrm{x}\}$ means 0 or more occurrences of $\mathrm{x}$, $\{\mathrm{x}\}_{1}{ }^{\mathrm{N}}$ means 1 or more occurrences of $\mathrm{x},()$ is used for grouping, | stands for "OR" and $[\mathrm{x}]$ denotes that $\mathrm{x}$ is optional. The following words contain only terminal symbols: Phrase, Verb, Predicate, Name, Action-Verb, Linking-Verb, Letter, and 
Digit. The following words and phrases are terminal symbols: TITLE, GOAL, CONTEXT, RESOURCE, ACTOR, EPISODES, EXCEPTION, GEOGRAPHICAL LOCATION, TEMPORAL LOCATION, PRECONDITION, POST-CONDITION, CONSTRAINT, IF, THEN, WHILE, DO, AND, OR, MUST, NOT, “[" and "]". Figure 19 shows how a scenario is described using the terminal and non-terminal symbols described in Table 13.

Our scenario grammar assumes that an episode sentence and exception solution are declared according to the format "[Actor | Resource] + Action-Verb + [Direct-Object-Predicate]", where "Action-Verb" express action and are the most common verbs in the present tense, and "Direct-Object-Predicate" refers to an object affected by the action. In the sentence "The Customer submits the Order", the work "submits" is an Action-Verb and the word "Order" is the Direct-Object-Predicate.

For example, a simple episode is described as follows:

$<\mathrm{Id}>(([$ Actor $\mid$ Resource $]+$ Action-Verb + [Direct-Object-Predicate] $) \mid$ $<$ Title $>)+$

[PRE-CONDITION: $<$ Pre-condition $>$ ] +

[POST-CONDITION: $<$ Post-condition $>]+$ [CONSTRAINT: $\{<$ Constraint $>\}$ ]

The first element of a simple episode is the identifier. The second element is a Sentence that describes a situation involving users, system, environment or system's components. Optionally; the other elements are non-functional requirements (Constraint) related to the episode, the initial state (Pre-condition) before the episode is carried out, and the expected results (Post-condition) after the episode occurs.

An exception is described as follows:

$<$ Id $>$ IF $<$ Cause $>$ THEN $(([$ Actor $\mid$ Resource $]+$ Action-Verb $+[$ DirectObject-Predicate]) $\mid<$ Title $>$ ) +

[POST-CONDITION: $<$ Post-condition> ] +

The first element of an exception is the identifier. This is composed by the identifier of the episode followed by the number of the exception (an episode can branch several exceptions). The second element is the Cause that triggers the exception, the third element is the Solution to treat the exception, and optionally, the Post-condition attribute is the expected results after performing the Solution. 
A condition may be formally defined as a logical sentence declared according to the format "(Actor | Resource) + Linking-Verb + Predicate". In linguistics (Cambridge, 2015), a "Linking-Verb" (copular verb) is a word used to link the Subject (Actor or Resource) of a sentence with a Predicate (a subject complement), such as the word "is" in the sentence "Feeder area is available".

Linking verbs are not followed by objects. Instead, they are followed by phrases which give extra information about the subject (e.g. noun phrases, adjective phrases, adverb phrases or prepositional phrases). Linking verbs include the conjugated form of limited number of verbs: Be, Look, Feel, Taste, Smell, Sound, Seem, Appear, Get, Become, Grow, Stay, Keep, Turn, Prove, Go, Remain, Resemble, Run, Lie (Usingenglish, 2015).

Like condition, a State (pre-condition and post-condition) may be formally defined as a sentence declared according to the format "(Actor | Resource) + State-Verb + Predicate". In linguistics (Grammaring, 2015), a "State-Verb" express a state which is relatively static. They include verbs of perception, cognition, the senses, emotion and state of being. In the sentence "The buffer is empty", the work "is" is a State-Verb and the word "empty" is the Predicate. State verbs are not normally used in continuous forms. Examples of state verbs include: Appear, Be, Believe, Belong, Consider, Consist, Contain, Cost, Doubt, Exist, Fit, Hate, Hear, Have, Know, Like, Love, Matter, Mean, Need, Owe, Own, Prefer, Remember, Resemble, Seem, Suppose, Suspect, Understand, Want, Wish.

\subsection{3.}

\section{Scenario Relationships-based Modularity}

When facing large systems, the number of scenarios could be unmanageable and the requirements engineers become sunk in details, losing the global vision of the system. Or simply, the requirements engineers are most likely interested in a subset of scenarios. In order to face this problem, Leite et al. (2000) proposes the construction integration scenarios based on the existing scenarios. An integration scenario gives an overview of the relationship among several scenarios of the system, since each integration scenario episode corresponds to a sub-scenario. A sub-scenario details in another scenario a complex episode sentence.

Thus, the scenario language is designed with modularity in mind, mainly using a mereology operator for decomposition and the construction of integration 
scenarios. Modularity is considered a mechanism to deal with the scenario explosion problem (Lee et al., 1998; Leite et al., 2000).

\subsubsection{1.}

Sequential Relationships

Besides of integration scenario, other relationships (pre-condition, postcondition, sub-scenario, exception and constraint) also provide modularity through the inter-connectivity among related scenarios. For example, the comprehension of an episode is facilitated by the use of natural language, wellbounded situations, and mainly through the use of sub-scenarios, i.e., an episode sentence may be detailed in another scenario, or an exception may be treated by another scenario.

A scenario element is detailed in another scenario when (Leite et al., 2000):

- Common behavior is detected in several scenarios;

- A complex conditional or alternative course of action appears in a scenario; and

- The need to enhance a situation with a concrete and precise goal is detected inside a scenario.

Through these relationships it is possible to determine the order in which the scenarios should be executed. For instance, if the scenario $\boldsymbol{X}$ has among its preconditions the scenario $\boldsymbol{Y}$, then the last one must be executed first (precedence order).

In a scenario description, if we include the title of another scenario (UPPERCASE sentence) within the context (pre-condition or post-condition), an episode (sentence), an exception (solution) or a constraint, then, this context subcomponent, episode, exception or constraint will be detailed or treated by this last scenario. Thus, the scenario language defines semantics to represent sequential relationships among scenarios. Scenarios can be connected to other scenarios through links or references, yielding a complex network of relationships:

- Pre-condition is a relationship defined within the context element of a scenario. If a scenario has among its pre-conditions another scenario, then the last one must be executed first. 
- Post-condition is a relationships defined within the context element of a scenario. If a scenario has among its post-conditions another scenario, then the last one must be executed last.

- Sub-scenario relationship is defined when an episode (sentence) of a scenario can be described by another scenario. This allows the decomposition of complex scenarios, facilitating both its writing and understanding.

- Exception relationship is defined when a scenario is used to detail the treatment of an exception (solution); the scenario that treats the exception is only executed when exception's cause is triggered in the main scenario.

- Constraint relationship is defined when a scenario is used to detail nonfunctional aspects that qualify/restrict the proper execution of another, which also give us an order among the scenarios.

\subsubsection{2.}

\section{Non-sequential Relationships}

Often in software development processes, multiple stakeholders are involved, with different needs, assumptions and points of view. But, a given group of stakeholders can be most likely interested in a specific subset of scenarios. According to Lee et al. (1998), although such subsets of scenarios might seemingly be independent, they are rarely truly independent in practice. Thus, scenarios also interact by complex non-sequential relationships, and in some cases these relationships are non-explicit.

So, scenarios are also related to other scenarios by explicit and non-explicit non-sequential relationships.

Explicit non-sequential relationships among scenarios are described using the structure for grouping non-sequential episodes (\#<episodes series $>\#$ ); i.e., if a set of episodes inside a non-sequential group are detailed in another scenarios (sub-scenario relationship), then these sub-scenarios could be executed in an indistinct order or concurrently. In Figure 22, the episodes 10 and 11 of the main execution flow reference sub-scenarios described in Figure 23. These subscenarios are explicitly described to be executed in an indistinct order or concurrently. 
In some cases, the given scenarios could interact by non-explicit and nonsequential relationships; often, they communicate by concurrency, which can lead to erroneous situations such as deadlocks. From the concurrency perspective, a set of scenarios can be considered as a set of concurrently executing threads, and they could interact or compete with each other by communication channels or shared resources.

In practice, it is very difficult to identify non-sequential relationships among scenarios, because most of the proposed languages to write scenarios do not provide:

- Constructs or semantics to represent explicitly the relationships among scenarios;

- Heuristics to find non-explicit relationships based on concurrency characteristics (e.g. non-determinism and synchronization by shared resources);

- Heuristics to assist the developer in making explicit non-sequential relationships.

\subsubsection{3.}

\section{Heuristics to Find Non-explicit and Non-sequential Relationships}

An heuristic for finding non-explicit relationships is shown in this sub section. It uses information of scenario descriptions and the scenario model for making explicit non-sequential relationships among scenarios.

This heuristic could assist the developers in identifying concurrency opportunities since initial requirements engineering activities, and requirement engineers in detecting defects arose from interactions among related scenarios.

In a concurrent system, local processes are first developed, and it has particular characteristics such as non-deterministic execution and synchronization between processes. These characteristics arise from the possibility of communication between process, which can be via communication channels or shared resources, resulting in complex interactions.

In the scenario language, two or more scenarios are likely related when they share common portions in their descriptions, i.e., they involve the participation of common actors, they access shared resources or they are executed in the same context. Leite et al. (2005) used the concept of Proximity Index to more detailed 
comparisons between any two scenarios with obscure and poorly defined borders. It is defined by:

$$
\text { Let } \mathbf{I}_{\mathrm{ij}}=\left(\boldsymbol{\alpha} * \mathbf{C} \cap_{\mathrm{ij}}+\boldsymbol{\beta} * \mathbf{A} \cap_{\mathrm{ij}}+\Upsilon * \mathbf{R} \cap_{\mathrm{ij}}\right) /\left(\boldsymbol{\alpha} * \mathbf{C} \cup_{\mathrm{ij}}+\boldsymbol{\beta} * \mathbf{A} \cup_{\mathrm{ij}}+\Upsilon * \mathbf{R} \cup_{\mathrm{ij}}\right)
$$

be the proximity index of Scenarios $\mathbf{S}_{\mathbf{i}}$ and $\mathbf{S}_{\mathbf{j}}$; where:

$\alpha, \beta, \Upsilon$ are weight factors.

$$
\mathbf{C} \cap_{\mathrm{ij}}=\mid \text { Context }\left(\mathrm{S}_{\mathrm{i}}\right) \cap \operatorname{Context}\left(\mathrm{S}_{\mathrm{j}}\right) \mid \text {; }
$$$$
\mathbf{A} \cap_{\mathrm{ij}}=\left|\operatorname{Actor}\left(\mathrm{S}_{\mathrm{i}}\right) \cap \operatorname{Actor}\left(\mathrm{S}_{\mathrm{j}}\right)\right| \text {; }
$$$$
\mathbf{R} \cap_{\mathrm{ij}}=\left|\operatorname{Resource}\left(\mathrm{S}_{\mathrm{i}}\right) \cap \operatorname{Resource}\left(\mathrm{S}_{\mathrm{j}}\right)\right| \text {; }
$$$$
\mathbf{C}_{\mathrm{ij}}=\mid \text { Context }\left(\mathrm{S}_{\mathrm{i}}\right) \cup \text { Context }\left(\mathrm{S}_{\mathrm{j}}\right) \mid \text {; }
$$$$
\mathbf{A} \cup \text { ij }=\left|\operatorname{Actor}\left(S_{i}\right) \cup \operatorname{Actor}\left(S_{j}\right)\right| ;
$$$$
\mathbf{R} \cup_{\mathrm{ij}}=\left|\operatorname{Resource}\left(\mathrm{S}_{\mathrm{i}}\right) \cup \operatorname{Resource}\left(\mathrm{S}_{\mathrm{j}}\right)\right| \text {; }
$$

$\operatorname{Actor}\left(\boldsymbol{S}_{\boldsymbol{k}}\right)$ : Actors of scenario k;

Resource $\left(\boldsymbol{S}_{\boldsymbol{k}}\right)$ : Resources of scenario $\mathbf{k}$;

Context $\left(\boldsymbol{S}_{\boldsymbol{k}}\right)$ : Context of scenario $\mathbf{k}$.

As the first step of the heuristic for finding non-explicit non-sequential relationships among scenarios (see Heuristic 1), we filter sequentially and explicit non-sequentially related scenarios.

As the second step of the Heuristic 1, we adapted the Proximity Index among any two scenarios defined in (Leite et al., 2005), by considering only common actors or shared resources.

In this case, actors and resources have the same importance because two scenarios might interact by common actors or shared resources $(\boldsymbol{\beta}=\boldsymbol{\Upsilon}=\mathbf{1})$. Thus, if two scenarios have common actors or share resources, then, they could be related to each other. If the Proximity Index is higher or equal than 0.5, then there is an indication that scenarios need to be compared in a more detailed way.

Let $\mathbf{I}_{\mathbf{i j}}=\mathbf{M A X}\left(\left(\mathbf{A} \cap_{\mathrm{ij}} / \mathbf{A} \cup_{\mathrm{ij}}\right),\left(\mathbf{R} \cap_{\mathrm{ij}} / \mathbf{R} \cup_{\mathrm{ij}}\right)\right)$ be the proximity index of Scenarios $\mathbf{S}_{\mathbf{i}}$ and $\mathbf{S}_{\mathbf{j}}$; where:

$\operatorname{MAX}(\mathrm{x}, \mathrm{y})$ : Find maximum of $x$ and $y$.

As the third step of this heuristic, each pair of two scenarios with higher proximity index is compared in more detail. This comparison is needed to determine whether they interact by non-determinism or synchronization constraints; that is: 
- Non-determinism: It compares pre-conditions to determine whether there is a non-deterministic execution or not. For example, when a precondition described in a scenario $\mathbf{S}_{\mathbf{i}}$ appears like pre-condition in another scenario $\mathbf{S}_{\mathbf{j}}$, then, $\mathbf{S}_{\mathbf{i}}$ and $\mathbf{S}_{\mathbf{j}}$ might interact concurrently.

- Synchronization: It compares pre-conditions against post-conditions to determine whether there is synchronization or not. For example, when a pre-condition described in a scenario $\mathbf{S}_{\mathbf{i}}$ appears like post-condition in another scenario $\mathbf{S}_{\mathbf{j}}$, and a pre-condition described in $\mathbf{S}_{\mathbf{j}}$ appears like postcondition in $\mathbf{S}_{\mathbf{i}}$, then, $\mathbf{S}_{\mathbf{i}}$ and $\mathbf{S}_{\mathbf{j}}$ might interact concurrently.

In Heuristic 1 (Figure 20), we list some general criteria to make explicit potentially concurrent scenarios $\left(\mathbf{S}_{\mathbf{i}}\right.$ and $\left.\mathbf{S}_{\mathbf{j}}\right)$, since non-deterministic and synchronization perspectives. In order to compare two scenario elements (e.g. two goals), or verify the similarity between an item and the items of a set (e.g. intersection between two set of pre-conditions), we use Levenshtein's distance (Levenshtein, 1966).

In Heuristic 1:

- Seq-Related $\left(\boldsymbol{S}_{i}, \boldsymbol{S}_{j}\right)$ : scenario $\mathbf{i}$ and scenario $\mathbf{j}$ are sequentially related by: pre-condition, post-condition, constraint, sub-scenario or exception.

- Explicit-Non-Seq-Related $\left(\boldsymbol{S}_{\boldsymbol{i}}, \boldsymbol{S}_{\boldsymbol{j}}\right)$ : scenario $\mathbf{i}$ and scenario $\mathbf{j}$ are nonsequentially related by non-sequential group construct: \#<episode series $>\#$.

- Pre-Cond $\left(\boldsymbol{S}_{\boldsymbol{k}}\right)$ : \{pre-conditions in the context of scenario $\mathbf{k} \cup$ preconditions in the episodes of scenario $\mathbf{k}\}$;

- Post-Cond $\left(\boldsymbol{S}_{\boldsymbol{k}}\right)$ : \{post-conditions in the context of scenario $\mathbf{k} \cup$ postconditions in the episodes of scenario $\mathbf{k}\}$; 


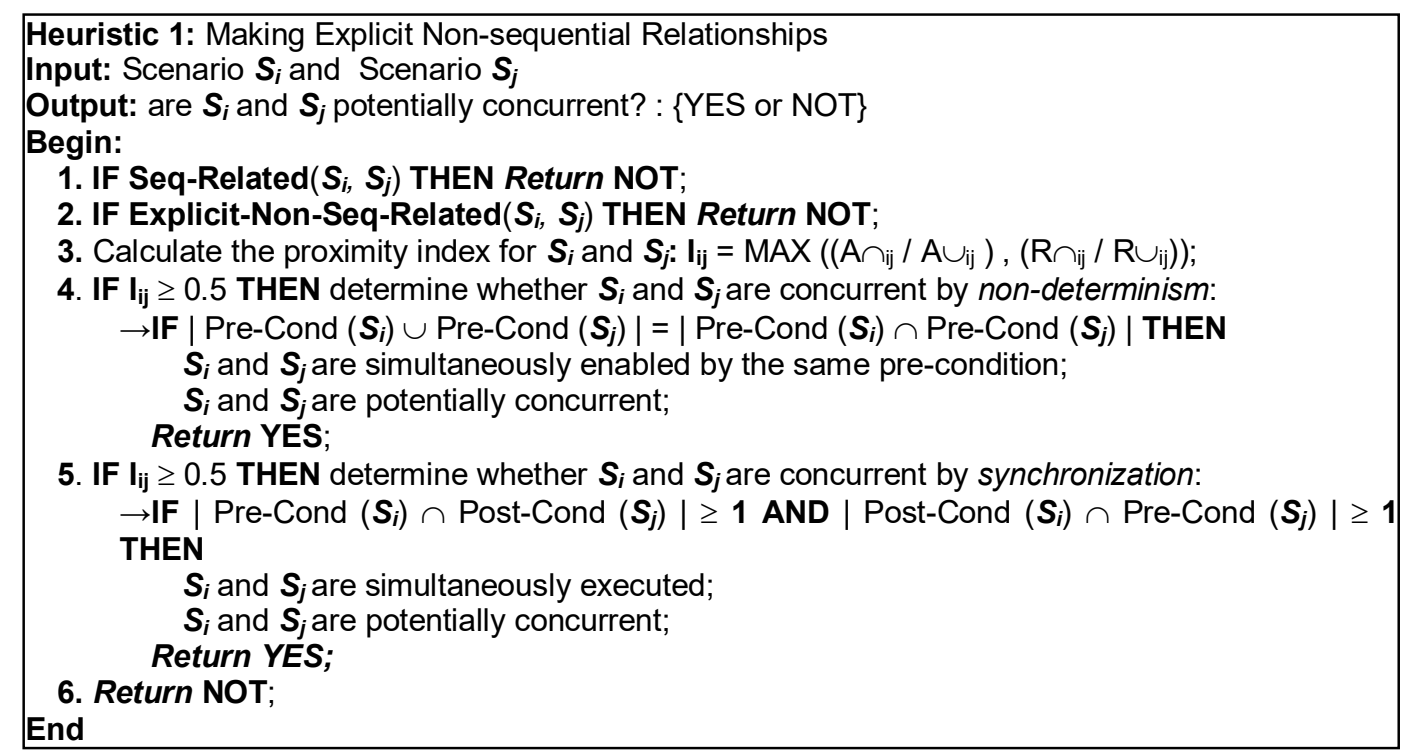

Figure 20 - Making Explicit Non-sequential Relationships (Heuristic 1).

In order to improve the reliability of systems initially specified as scenarios representations, the identification of non-explicit relationships among scenarios makes it possible to perform rigorous analysis focusing on related scenarios and achieve a more consistent and more correct requirements specification. This is especially important for systems involving concurrent, asynchronous, distributed, non-deterministic or parallel processes, such as distributed web services, multiagent systems, manufacturing systems or shared memory-based systems.

\subsection{4. Running Example}

This section describes a set of scenarios for describing a system that involves sequential and non-sequential relationships.

In the Online Broker System, the Broker System interacts with its partner services: Local Supplier and International Supplier. The system under consideration is an Online Broker System. The goal of the system is to allow customers to find the best supplier for a given order. A customer fills up an online order form and after submission; the system broadcasts it to local and international suppliers. Each supplier after examining the order may decide to decline or submit a bid. A local supplier needs to add taxes to the order total, while an international supplier needs to ensure an order does not include items restricted for export. Submitted bids are sent back to the broker to be shown to the customer, who eventually asks the system to proceed with a bid. The full 
scenarios of the "Online Broker System" example are shown in (Somé, 2010) using a use case language based on Cockburn's template (Cockburn, 2001).

In order to understand the execution order of a set of related scenarios, it is necessary to identify the main scenario of this set (or main scenarios). A main scenario will be the scenario that does not require any other scenario of the set, or that reference in its description to other scenarios. According to Almentero et al. (2014), we first determine the relationship between the scenarios of the set, and from identified relationships we will establish an execution order between them.

In the "Online Broker System", the Submit Order scenario is a main scenario because it precedes all others, and it is related to other scenarios by sequential and explicit non-sequential relationships. In the original version shown in (Somé, 2010), it is not obvious to perceive the relationships among related scenarios and that Process Bids scenario is a scenario executed after querying the customer (See Figure 21). The Process Bids scenario (Figure 23) is referenced inside Supplier scenarios (Somé, 2010). Other relationship that is difficult to perceive is the sequential relationship between "Register Customer" (Figure 23) and the main scenario "Submit Order". The meaning of the relationship is that scenario "Register Customer" extends scenario "Submit order" when condition "Customer is not registered" holds.

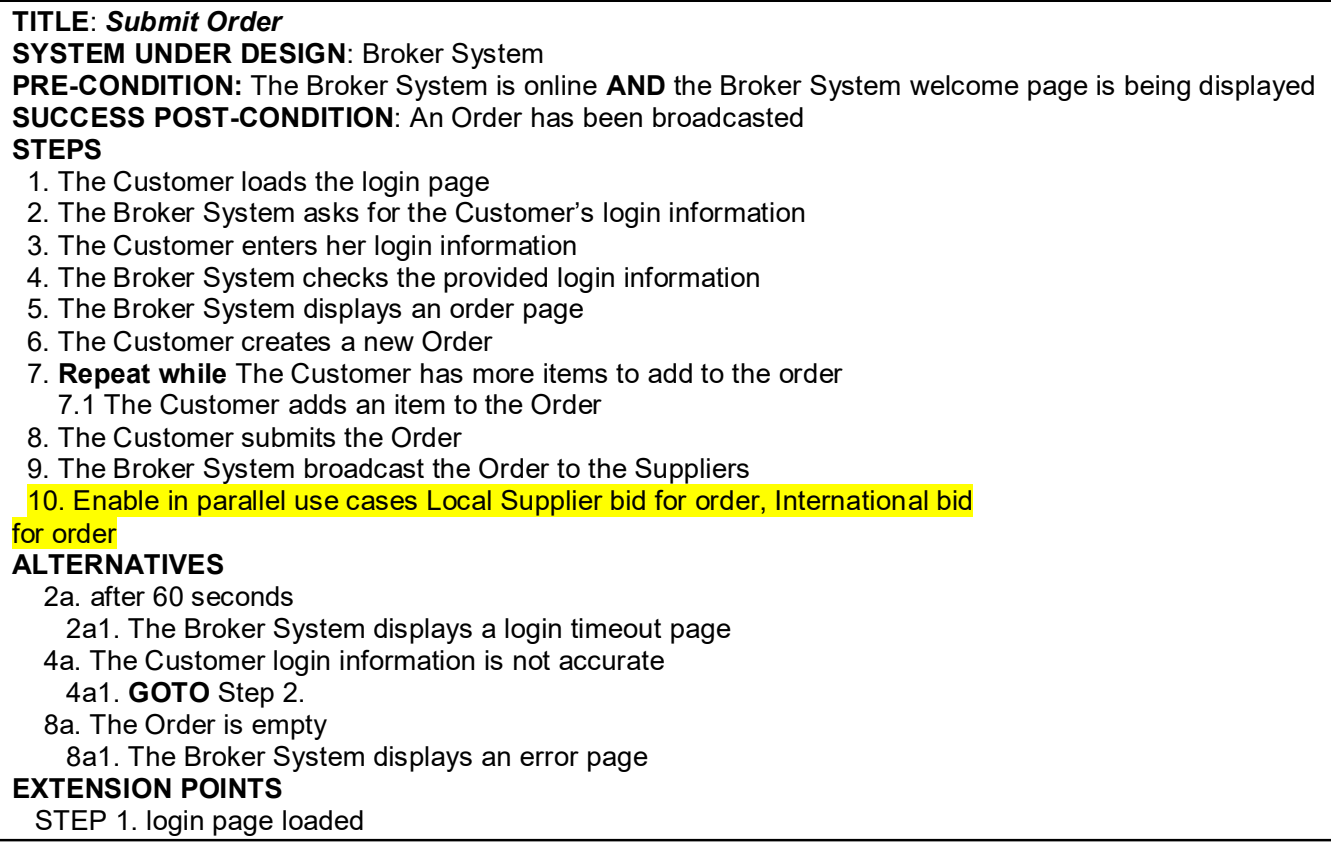

Figure 21 - "Submit Order" use case in the Online Broker System (Somé, 2010).

Using the scenario language proposed in this work, we re-described the Submit Order scenario to make explicit the sequential relationships by: (1) adding 
a last episode, which references the Process Bids scenario through sub-scenario relationship (See Figure 22); and (2) mapping the extension point into an exception with references the Register Customer scenario through exception relationship.

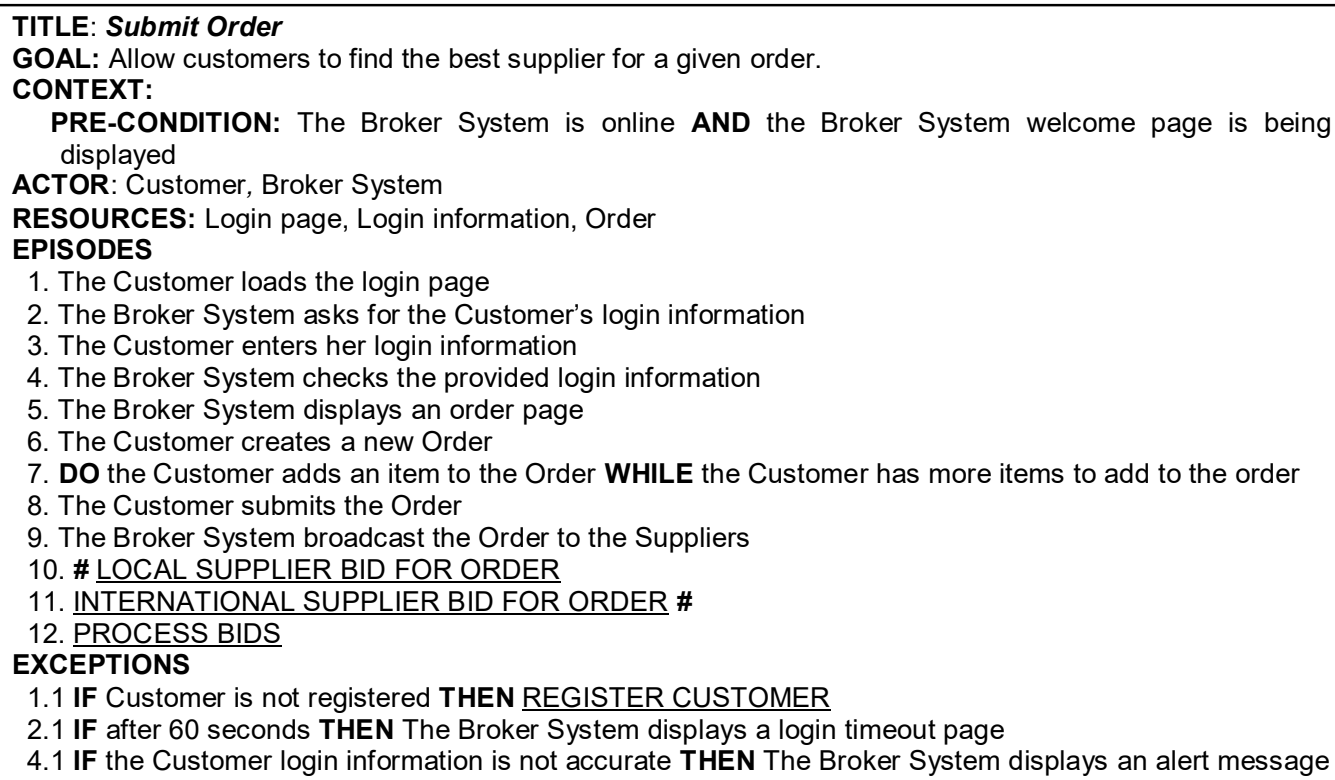

Figure 22 - Description of scenario "Submit Order" in the Online Broker System.

In Figure 22, the episodes 10 (LOCAL SUPPLIER BID FOR ORDER), 11 (INTERNATIONAL SUPPLIER BID FOR ORDER), 12 (PROCESS BIDS), and exception 1.1 (REGISTER CUSTOMER) are detailed in another scenarios. Figure 22 shows the sequential interaction among scenarios by sub-scenario (PROCESS BIDS) and exception (REGISTER CUSTOMER) relationships, and nonsequential relationships by explicit concurrency construct (SUPPLIERS).

PROCESS BIDS, REGISTER CUSTOMER, LOCAL SUPPLIER BID FOR ORDER and INTERNATIONAL SUPPLIER BID FOR ORDER are presented in Figure 23 and detailed in Appendix 1. PROCESS BIDS references sequentially to HANDLE PAYMENT scenario. 


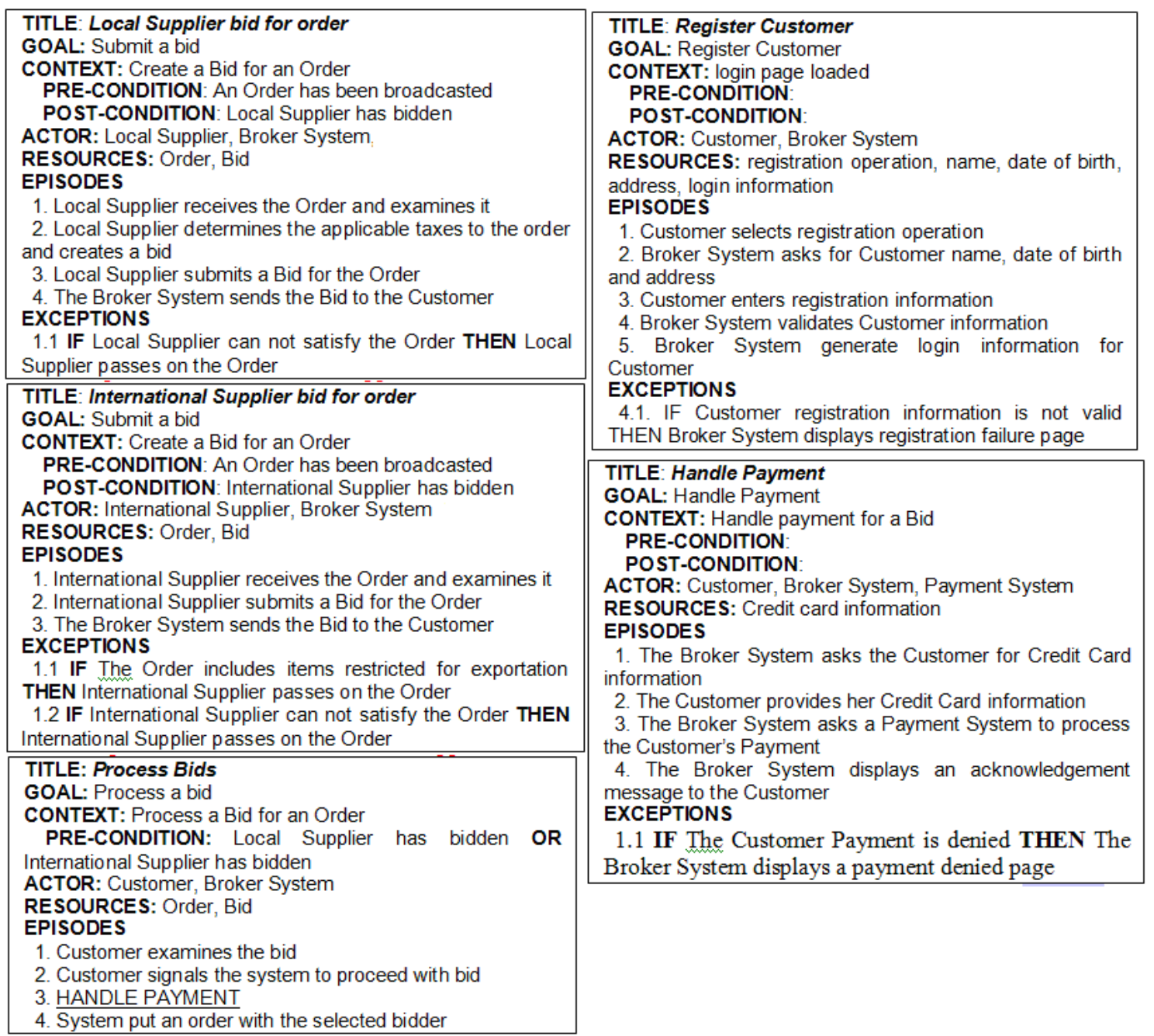

Figure 23 - Scenarios of the "Online Broker System".

In this example, from the main scenario (Submit Order), it is possible to identify the sequentially (PROCESS BIDS, REGISTER CUSTOMER) and explicit non-sequentially related scenarios (LOCAL SUPPLIER AND INTERNATIONAL SUPPLIER).

In most of projects, it is difficult to identify the non-explicit relationships among scenarios, mainly, non-sequential relationships among them.

For example, if we do not have any scenario referencing explicitly other scenarios, it will be difficult to perceive that Suppliers' scenarios are nonsequentially related to each one. So, in order to identify non-explicit relationships of "Online Broker System" scenarios, we apply the heuristic for finding nonexplicit relationships (described in Heuristic 1) to explore any two potentially related scenarios.

As the first step of the heuristic, we calculate the proximity index among any two scenarios. For example, we chose "Local Supplier for Bid" $\left(\mathbf{S}_{\mathbf{1}}\right)$ and 
"International Supplier for Bid" $\left(\mathbf{S}_{\mathbf{2}}\right)$ to be explored, and they have a degree of proximity high (proximity index $=1$ ). Therefore, they must be analyzed more deeply.

As the second step, we detect that they are enabled by the same precondition, and then they are non-sequentially related by non-determinism feature.

Table 14 shows the results of proximity index (equal to 1 ) and scenarios $\mathbf{S}_{\mathbf{1}}$ and $\mathbf{S}_{\mathbf{2}}$ are related by non-explicit non-sequential relationships (Non-determinism).

Table 14 - Proximity Index between Scenarios of the Online Broker System

\begin{tabular}{|c|c|c|c|c|c|c|c|c|c|c|}
\hline \multirow[t]{2}{*}{$S_{i}$} & \multirow[t]{2}{*}{$\mathbf{S}_{\mathrm{j}}$} & \multirow[t]{2}{*}{$\mathbf{A} \cap_{\mathrm{ij}}$} & \multirow[t]{2}{*}{$A \cup_{i j}$} & \multirow[t]{2}{*}{$\mathbf{R} \cap_{i j}$} & \multirow{2}{*}{$R \cup_{i j}$} & \multirow[t]{2}{*}{$\mathbf{I}_{\mathrm{ij}}$} & \multicolumn{3}{|c|}{ Non-determinism } & Synchronization \\
\hline & & & & & & & Goal & Temp_Loc & Pre-Condition & $\begin{array}{l}\text { Pre-Condition \& } \\
\text { Post-Condition }\end{array}$ \\
\hline & $\mathrm{S}_{2}$ & 2 & 4 & 2 & 2 & 1 & Similar & --- & YES & \\
\hline
\end{tabular}

In Figure 23, the "Local Supplier for Bid" and "International Supplier for Bid" specify as common pre-condition the availability of the "An order has been broadcasted". Thus, these scenarios interact by shared resources.

We identified the non-explicit relationships, because they can be used to perform early concurrent (potentially concurrent) system analysis to detect potential defects due to concurrency at early software development activities.

\section{2.}

\section{Pre-processing Scenarios}

In order to improve the efficacy of scenario transformation algorithm and the accuracy of NLP analysis tools, it is necessary to remove the irrelevant information and formatting symbols, such as URLs, HTML tags, parenthesized comments and bullets. According to Liu et al. (2014), the noise from the input document may affect the parsing accuracy. This is a general process applicable to any document.

Therefore, the steps to clean scenarios of these possible noise elements are described below:

- Removal of Empty Line: There is no empty line in the scenario.

- Removal of Capitalization: often it is convenient to lower case every character.

- Removal of Brackets: Text between brackets within a sentence is replaced by empty character. There are various bracket symbols: Parentheses “()", Square Brackets "[]" and Curly Braces " \{\} ". 
- Removal of URLs: URLs and hyperlinks within a sentence like comments or reviews should be removed.

- Removal of HTML Markup: HTML tags within a sentence should be removed.

- Removal Punctuation: For NLP analysis, all the punctuation marks and bullets according to the priorities should be dealt with. For example: "!”, “\#”, “?”, “॰” are important punctuations that need to be removed and replaced by a white space character.

- Apostrophe Lookup: According to Bansal (2014), to avoid any word sense disambiguation in text, it is recommended to maintain proper structure in it and to abide by the rules of context free grammar. When apostrophes are used, chances of disambiguation increase. For example "it's is a contraction for it is or it has".

We utilized regular expression matching to perform the filtering tasks. The last step (Apostrophe Lookup) was not considered in our pre-processing process because it is fairly domain dependent and a challenging topic in NLP research.

\section{3. Deriving Petri-Nets}

After constructing scenarios, it is possible to automatically derive Petri-Net formal specifications. In our approach, each scenario sentence (imperative or declarative) is translated into a Petri-Net node (transition or place, respectively). These Petri-Net nodes are linked by arcs giving rise to a Petri-Net model. Each translated scenario defines components of the initial system design.

\subsection{1.}

\section{Transforming Scenarios into Petri-Nets}

We assume that a scenario $\mathbf{S}$ : (1) starts at an idle state with all necessary resources, pre-conditions or constraints; (2) performs a collection of partially ordered event occurrences (episodes or exceptions), each guarded by a set of conditions (pre-conditions, post-conditions, or causes) and restricted by a set of constraints; and (3) returns to the idle state releasing the resources, preconditions (if it is not returned by some previous event) or constraints after completion (adapted from Cheung et al. , 2006). 
A Petri-Net PN is derived from a scenario $\mathbf{S}$ as follows: We identify the event occurrences (episodes and exceptions) and their pre-conditions (or causes), constraints and post-conditions. For each event, a transition is created for denoting the location of event occurrence. Input places are created to denote the locations of its pre-conditions, causes and constraints (They restrict but do not impede - TRUE). Output places are created to denote the location of its postconditions. Event labels, condition labels and constraint labels are assigned to these transitions and places accordingly. The initial marking $\mathbf{M}_{\mathbf{0}}$ of the $\mathbf{P N}$ is then created to denote the initial state, in which tokens are added into input places that represent pre-conditions, causes or constraints. Execution of the scenario begins at this initial marking which semantically means the system initial state, including the availability of all resources, pre-condition, causes or constraints. It ends at the same marking that semantically means the release of these resources, preconditions, causes or constraints.

As the first step of the method for Transforming a Scenario into an Equivalent Petri-Net (Method 1 in Figure 26), we define mapping rules to translate scenario elements (Title, Goal, Context, Resource, Actor, Episodes, Exception) into Petri-Net elements (transition, place and arc).

For each scenario element, a sub Petri-Net which contains places, transitions and arcs is derived. The different mapping rules to derive a sub Petri-Net from a scenario element are described using a structure composed of left and right hand sides (LHS and RHS). LHS is the conditional part of the rule (scenario element), and RHS is the expected result of the rule (sub Petri-Net).

Table 15, Table 16, Table 17, Table 18 and Table 19 define the mapping rules for initial state, episodes, exception, concurrency constructs and final state of a scenario, respectively. In LHS side (Scenario), "e" is an episode and "ex" is an exception. In RHS side (Sub Petri-Net), " $\mathbf{t}$ " is a transition (with the name attribute), "p" is a place (with the name and number of tokens attributes) and "a" is an arc (with source and target attributes). Below, we detail these mapping rules.

In order to preserve the event sequences described within the main flow (episodes) and exceptional flows of a scenario, we add appropriate Input dummy place and Output dummy place to the sub Petri-Nets derived from scenario elements. These dummy places are used for linking sub Petri-Nets derived from sequential events (e.g. episode 1 and episode 2 of the main flow of episodes). 
A Dummy transition is added to the sub Petri-Nets derived from scenario initial state and final state. It represents an initial event or a final event derived from the main flow of episodes, i.e., a scenario initial state or final state is mapped into a sub Petri-Net composed of a dummy transition and its corresponding input and output dummy places.

Table 15 - Transforming Scenario Triggering

\begin{tabular}{|c|c|}
\hline Rule & Transform Scenario Triggering - Initial State \\
\hline & When \\
\hline LHS & Title, Resources, Context $=\{$ Constraint, Pre-condition $\}$ \\
\hline & Then \\
\hline $\begin{array}{l}\text { RHS } \\
\text { (sub } \\
\text { Petri-Net) }\end{array}$ & 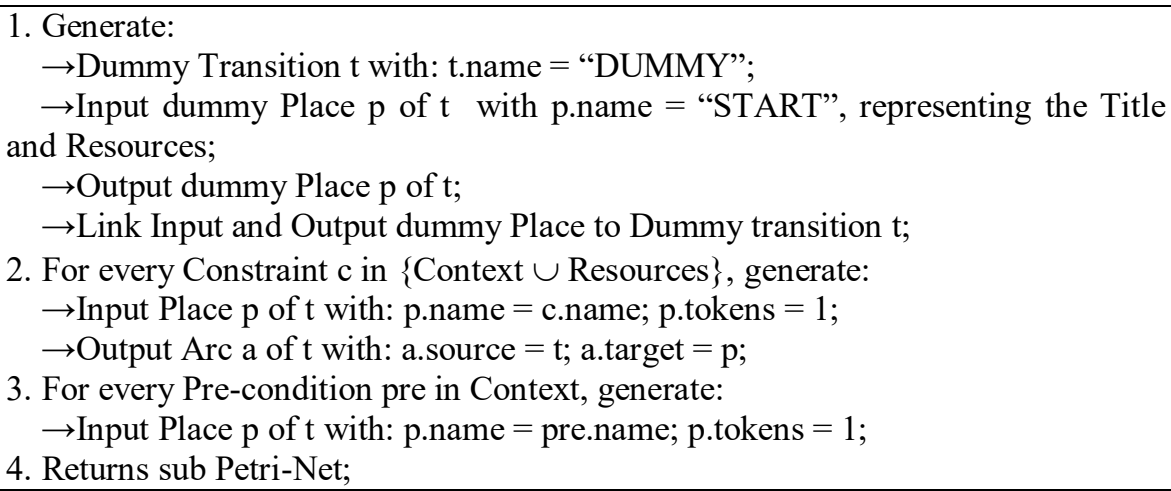 \\
\hline & End \\
\hline
\end{tabular}

Table 16 - Transforming Episode

\begin{tabular}{|l|l|}
\hline Rule & Transform Episode \\
\hline LHS & When \\
\hline Scenario) & Episode $\mathbf{e}=\{$ Id, Sentence, Type, Condition, Constraint, Pre-condition, Post- \\
& condition $\}$
\end{tabular}


Table 17 - Transforming Concurrency Construct

\begin{tabular}{|c|c|}
\hline Rule & Transform Concurrency Construct \\
\hline & When \\
\hline LHS (Scenario) & Episode $\mathbf{e 1}$ and Episode $\mathbf{e} \mathbf{2}$ \\
\hline & Then \\
\hline $\begin{array}{l}\text { RHS } \\
\text { (sub } \\
\text { Petri-Net) }\end{array}$ & $\begin{array}{l}\text { 1. IF e1.sentence starts with "\#”, generate: } \\
\rightarrow \text { Dummy Transition } \mathbf{t} \text { with: t.name = "FORK"; } \\
\rightarrow \text { Input dummy Place } \mathbf{p} \text { of } \mathbf{t} \text {; } \\
\rightarrow \text { Output dummy Place } \mathbf{p} \text { of } \mathbf{t} \text {; } \\
\rightarrow \text { Link Input and Output dummy Place to Dummy } \\
\text { transition t; } \\
\text { 2. IF e2.sentence ends with “\#”, generate: } \\
\rightarrow \text { Dummy Transition } \mathbf{t} \text { with: } \mathbf{t} \text {.name = "JOIN"; } \\
\rightarrow \text { Input dummy Place } \mathbf{p} \text { of } \mathbf{t} \text {; } \\
\rightarrow \text { Output dummy Place } \mathbf{p} \text { of } \mathbf{t} \text {; } \\
\rightarrow \text { Link Input and Output dummy Place to Dummy } \\
\text { transition t; } \\
\text { 3. Returns sub Petri-Net1 for } \mathbf{e} 1 \text { and Petri-Net2 for } \mathbf{e} 2 ;\end{array}$ \\
\hline & End \\
\hline
\end{tabular}

\begin{tabular}{|c|c|}
\hline Rule & Transform Exception \\
\hline & When \\
\hline \multirow[t]{2}{*}{ LHS (Scenario) } & Episode $\mathbf{e x}=\{$ Id, Cause, Solution, Post-condition $\}$ \\
\hline & Then \\
\hline $\begin{array}{l}\text { RHS } \\
\text { (sub } \\
\text { Petri-Nets) }\end{array}$ & $\begin{array}{l}\text { 1. Generate: } \\
\rightarrow \text { Transition } \mathbf{t} \text { with: t.name }=\mathbf{e x} \text {.solution; } \\
\rightarrow \text { Input dummy Place } \mathbf{p} \text { of } \mathbf{t} ; \\
\rightarrow \text { Output dummy Place } \mathbf{p} \text { of } \mathbf{t} \text {; } \\
\rightarrow \text { Link Input and Output dummy Place to Dummy } \\
\text { transition t; } \\
\text { 2. For every Cause } \mathbf{c} \text { in ex, generate: } \\
\rightarrow \text { Input Place } \mathbf{p} \text { of } \mathbf{t} \text { with: } \mathbf{p} \text {.name = c.name; } \mathbf{p} \text {.tokens }= \\
\text { 1; } \\
\text { 3. For every Post-condition post in ex, generate: } \\
\rightarrow \text { Output Place } \mathbf{p} \text { of } \mathbf{t} \text { with: } \mathbf{p} \text {.name = post.name; } \\
\text { 4. Returns sub Petri-Net; }\end{array}$ \\
\hline & \\
\hline
\end{tabular}

Table 19 - Transforming Scenario Completion

\begin{tabular}{|l|l|}
\hline Rule & Transform Scenario Completion - Final State \\
\hline & When \\
\hline LHS (Scenario) & Context = POst-condition $\}$ \\
\hline & Then \\
\hline RHS & 1. Generate: \\
Petri-Net) & $\rightarrow$ Dummy Transition $\mathbf{t}$ with: t.name = "DUMMY"; \\
& $\rightarrow$ Input dummy Place pof $\mathbf{t} ;$ \\
& $\rightarrow$ Output dummy Place pof $\mathbf{t}$ with p.name = "FINISH"; \\
& $\rightarrow$ Link Input and Output dummy Place to Dummy \\
& transition t; \\
& 2. For every Post-condition post in Context, generate: \\
& $\rightarrow$ Output Place p of t with: p.name = post.name; \\
& p.tokens = 1; \\
& 3. Returns sub Petri-Net; \\
& End \\
\hline
\end{tabular}

Figure 24 depicts the visual transformation (LHS $\rightarrow$ RHS) of a simple episode into Petri-Net elements. In this example, a simple episode (Submit Order 
Scenario) is mapped into a transition with an input dummy place and an output dummy place of the transition.

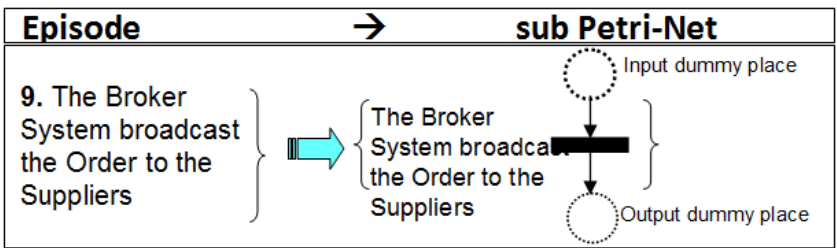

Figure 24 - Transforming Simple Episode

The visual transformation (LHS $\rightarrow$ RHS) from Scenario elements into PetriNet elements is depicted in

Figure 25. These transformations perform the tasks (mapping rules) defined in Table 15, Table 16, Table 17, Table 18 and Table 19.

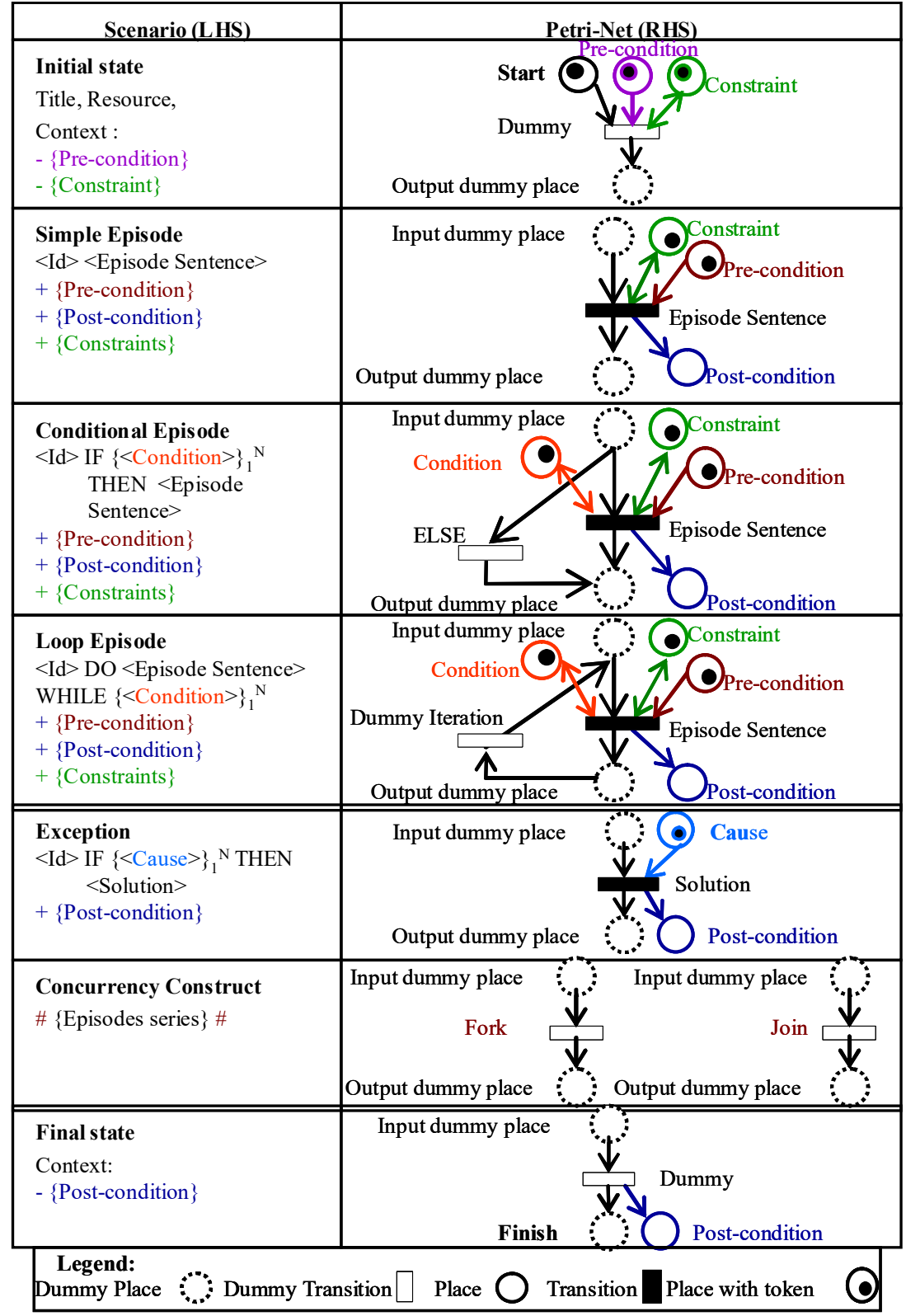

Figure 25 - Mapping scenario constructs into Petri-Net elements. 
As the second step of the Method 1 (Figure 26), the sub Petri-Nets generated from scenario elements are composed into a whole Petri-Net by Fusion Place or Modified Fusion Place operations.

Definition 6.1 (Fusion Place): A sub Petri-Net can be fused with other sub Petri-Net by fusing the output dummy place of the first sub Petri-Net into the input dummy place of the last sub Petri-Net.

Definition 6.2 (Modified Fusion Place): Any sub Petri-Net can be fused with other sub Petri-Net by fusing at least a common place among them. For example, two sub Petri-Nets derived from different episodes can be fused, if they have a common place that represents a common pre-condition.

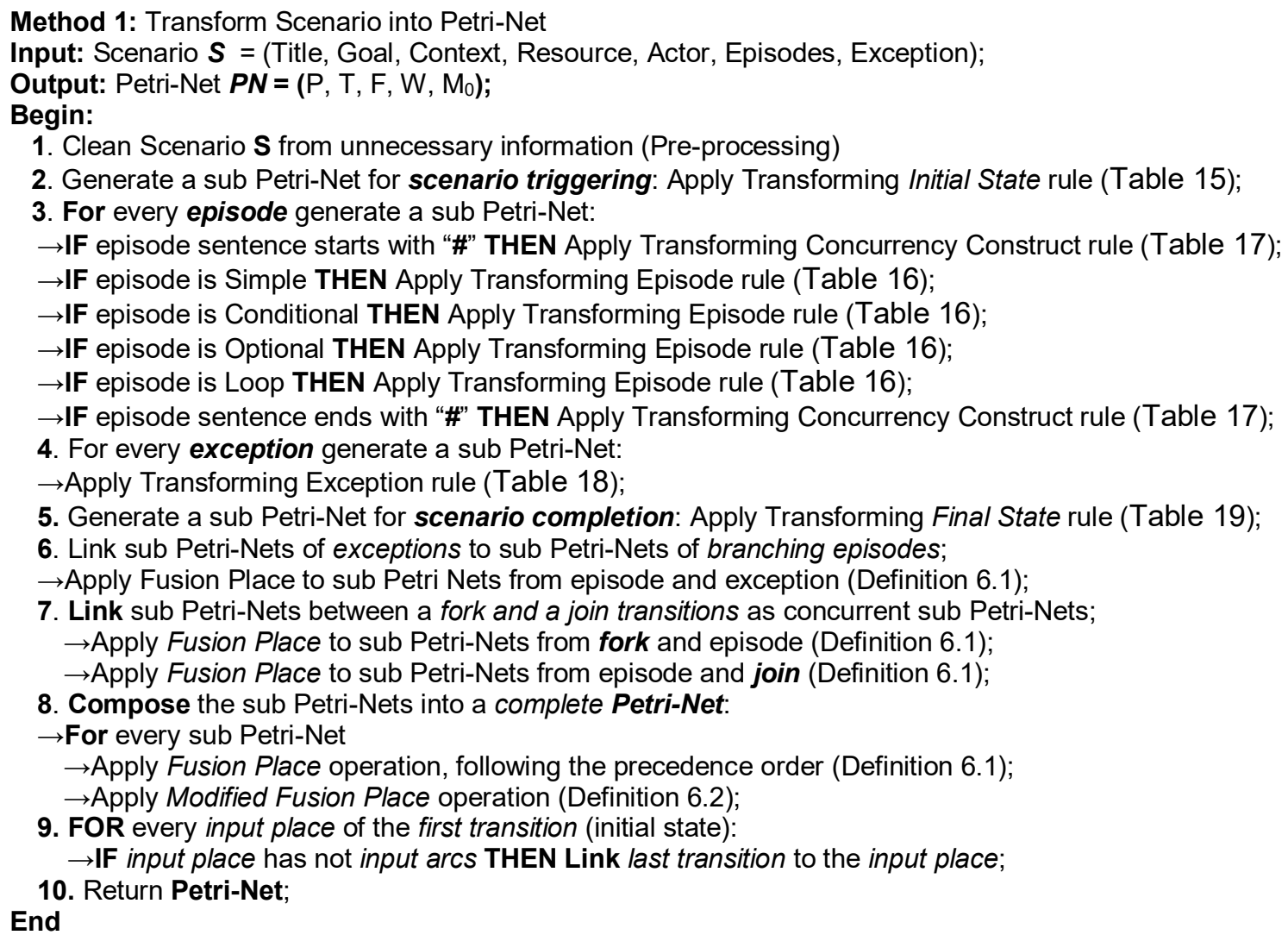

Figure 26 - Transform Scenario into Petri-Net (Method 1).

\subsection{2. Integrating Petri-Nets}

For every scenario and its related scenarios, we generate partial Petri-Nets in order to integrate these partial Petri-Nets into a consistent whole Integrated Petri-Net. The Integrated Petri-Net reflects exactly the original properties of the synthesized Petri-Nets (Demonstrated in Section 4.3.4). 
In the proposed scenario language, scenarios are related to other scenarios by explicit sequential relationships (pre-condition, post-condition, constraint, subscenario or exception). When a scenario is chosen to be a main scenario, and translated into a main Petri-Net, the referenced scenarios (sequentially) are mapped into input places (pre-conditions or constraints), output places (postconditions) or transitions (episodes' sentence or exceptions' solution).

In this case, a main scenario is the starting point to find the related scenarios.

As the first step of the method for integrating Petri-Nets (Method 2 in Figure 27), each sequentially related scenario is translated into a Petri-Net. After it, each one of these Petri-Nets must be replaced into the corresponding place or transition of the main Petri-Net. Our first step is the substitution of places or transitions.

Definition 6.3 (Substitution Transition): Any transition (not dummy) of a Petri-Net can be replaced by any other Petri-Net. Then the input dummy place of the transition is fused with the first input dummy place (Start) of the replacing Petri-Net and the output dummy place of the transition is fused with the last output dummy place (Finish) of the replacing Petri-Net.

Definition 6.4 (Substitution Input Place): Any input place (not dummy) of a Petri-Net can be replaced by any other Petri-Net. Then the last output dummy place (Start) of the replacing Petri-Net is fused with the input place.

Definition 6.5 (Substitution Output Place): Any output place (not dummy) of a Petri-Net can be replaced by any other Petri-Net. Then the first input dummy place (Start) of the replacing Petri-Net is fused with the output place.

Scenarios are also related to other scenarios by explicit and non-explicit non-sequential relationships (Indistinct order or Concurrency).

Explicit non-sequential relationships among scenarios are described using the structure for grouping non-sequential episodes (\#<episodes series $>\#)$. If a main scenario is mapped into a main Petri-Net, the explicit non-sequentially related scenarios (episodes between a Concurrency Construct) are mapped into transitions.

Non-explicit and non-sequential relationships among scenarios are found by analyzing common actors or shared resources (See Section 4.1). If a main scenario is mapped into a main Petri-Net, the interaction with non-explicit and 
non-sequentially related scenarios is described by common pre-conditions or postconditions, these common conditions are mapped into input places or output places.

As the second step of the method for integrating Petri-Nets (Method 2 in Figure 27), each non-sequentially related scenario is translated into a Petri-Net. Among the Petri-Nets, there are common places (with the same labels) that denote the same pre-condition or post-condition, and they need to be uniquely represented from the system point of view (Cheung et al., 2006). Our second step is basically the substitution of transitions (episodes referencing explicit nonsequential scenarios) and fusion of common places (non-explicit non-sequential scenarios interact by common conditions).

Definition 6.6 (Concurrent Fusion Place): Any Petri-Net can be fused with other Petri-Net by fusing at least a common place (from pre-condition or post-condition) among them.

Method 2: Integrate Petri-Nets

Input: Main Scenario $S=$ (Title, Goal, Context, Resource, Actor, Episodes, Exception);

Output: Integrated Petri-Net $I P N=\left(P, T, F, W, M_{0}\right)$

Begin:

1. Derive Main Petri-Net from the Main Scenario (Method 1);

2. Identify sequential relationships from the Main Scenario by Pre-condition. Post-condition, Constraint, Sub-scenario or Exception;

3. Identify explicit non-sequential relationships from the Main Scenario by analyzing Concurrency Constructs;

4. Identify non-explicit non-sequential relationships from the Main Scenario by common Pre-condition or Post-condition;

5. Obtain a whole Integrated Petri-Net from the Main Petri-Net:

$\rightarrow$ For every scenario in sequentially related scenarios:

$\rightarrow$ Transform scenario into a Petri-Net (Method 1);

$\rightarrow$ IF current Petri-Net represents a Sub-scenario or Exception in Main Scenario THEN:

$\rightarrow$ Substitute the corresponding "Transition" of the Main Petri-Net (Definition 6.3);

$\rightarrow$ IF current Petri-Net represents a Pre-Condition or Constraint in Main Scenario THEN

$\rightarrow$ Substitute the corresponding "Input Place" of the Main Petri-Net (Definition 6.4);

$\rightarrow$ IF current Petri-Net represents a Post-Condition in Main Scenario THEN:

$\rightarrow$ Substitute the corresponding "Output Place" of the Main Petri-Net (Definition 6.5);

$\rightarrow$ For every scenario in explicit non-sequentially related scenarios:

$\rightarrow$ Transform scenario into a Petri-Net (Method 1);

$\rightarrow$ Substitute the corresponding "Transition" of the Main Petri-Net (Definition 6.3);

$\rightarrow$ For every scenario in non-explicit non-sequentially related scenarios:

$\rightarrow$ Transform scenario into a Petri-Net (Method 1);

$\rightarrow$ Fuse the common places between the current Petri-Net and Main Petri-Net (Definition 6.6);

End

6. Return integrated Main Petri-Net;

Figure 27 - Integrate Petri-Nets (Method 2).

4.3.3.

\section{Petri-Net Example}

For illustration, we applied the Methods 1 and 2 (Figure 26 and Figure 27)

to obtain the Petri-Nets and Integrated Petri-Nets of the "Online Broker System". 
In the "Online Broker System", we choose the "Submit Order" scenario as main scenario because it does not require any other scenario of the set of scenarios and references to the most of scenarios of the system. By applying the Method 1 (Figure 26), we obtain the Petri-Net for the "Submit Order" scenario. It was derived by mapping the scenario components of the main execution flow episodes and exceptions. Figure 22 and Figure 23 depict the set of scenarios of the "Online Broker System", and Figure 28 (b) shows the Petri-Net for the "Submit Order" scenario.

For "Submit Order" scenario (Figure 22 and Figure 23), 16 event occurrences are identified (12 in the main flow - episodes and 4 in the exceptional flows): T1 (The Customer loads the login page), T2 (The Broker System asks for the Customer login information), T3 (The Customer enters her login information), T4 (The Broker System checks the provided login information), T5 (The Broker System displays an order page), T6 (The Customer creates a new Order), T7 (The Customer adds an item to the Order), T8 (The Customer submits the Order), T9 (The Broker System broadcast the Order to the Suppliers), T10 (LOCAL SUPPLIER BID FOR ORDER), T11 (INTERNATIONAL SUPPLIER BID FOR ORDER), T12 (PROCESS BIDS), T1.1 (REGISTER CUSTOMER), T2.1 (The Broker System displays a login timeout page), T4.1 (The Broker System displays an alert message) and T8.1 (The Broker System displays an error message). We construct a Petri-Net by creating transitions T1, T2,.., T11, T12 and T13 to denote these events and appending to each transition input and output places to denote: (1) internal dummy input and output places, or (2) input conditions (exception's cause or episode's condition) and post-conditions. Additionally: (1) two dummy transitions (Fork1 and Join1) are created for synchronization of concurrent transitions T10 and T11; and (2) two dummy transitions are created to denote the scenario triggering (T0) and the scenario completion (T13). 


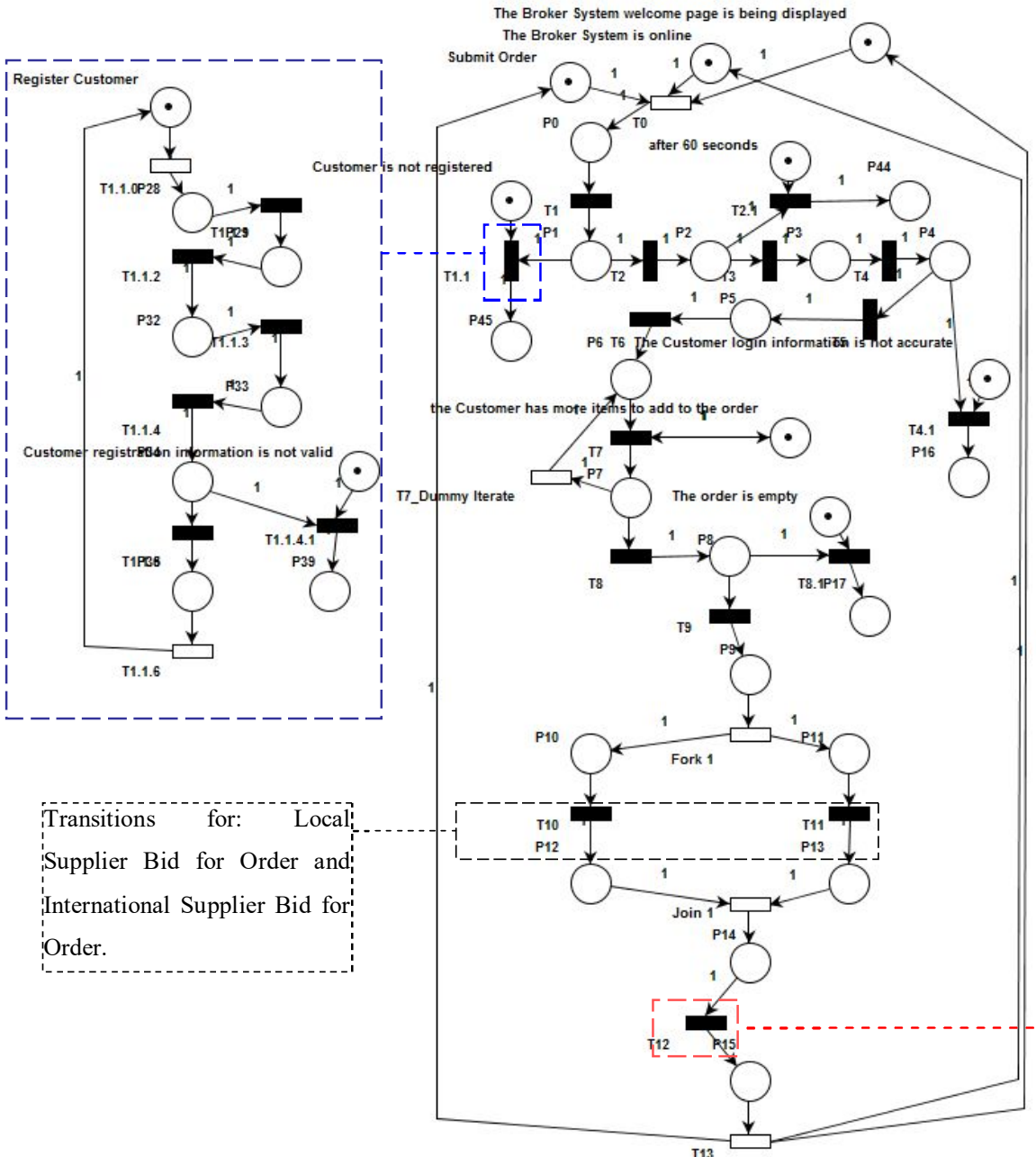

a)

b)

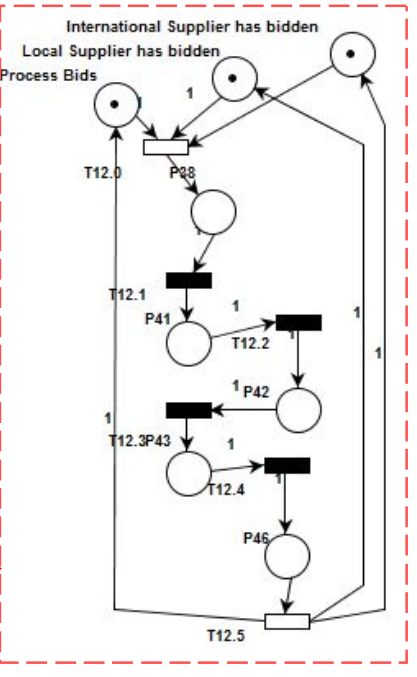

c)

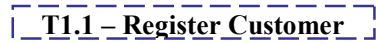

Legend:

Dummy Transition $\square$ Place $\bigcirc$ Transition $\square$ Place with token

Legends for Transition Labels

T1 The Customer loads the login page)

T2 The Broker System asks for the Customer login information

T3 The Customer enters her login information

T4 The Broker System checks the provided login information

T5 The Broker System displays an order page

T6 The Customer creates a new Order

T7 The Customer adds an item to the Order

T8 The Customer submits the Order

T9 The Broker System broadcast the Order to the Suppliers

T10 LOCAL SUPPLIER BID FOR ORDER

T11 INTERNATIONAL SUPPLIER BID FOR ORDER

T12 PROCESS BIDS

T1.1 REGISTER CUSTOMER

T2.1 The Broker System displays a login timeout page

T4.1 The Broker System displays an alert message

T8.1 The Broker System displays an error message
Legends for Transition Labels

T1.1.1 Customer selects registration operation

T1.1.2 Broker System asks for Customer name, date of birth and address

T1.1.3 Customer enters registration information

T1.1.4 Broker System validates Customer information

T1.1.5 Broker System generate login information for Customer

T1.1.4.1 Broker System displays registration failure page

T12.1 Customer examines the bid

T12.2 Customer signals the system to proceed with bid

T12.3 HANDLE PAYMENT

T12.4 System put an order with the selected bidder

Figure 28 - Register Customer (a), Submit Order (b) and Process Bids (c) PetriNets.

Revisiting the "Submit Order" scenario, exception 1.1 and episodes 10, 11 and 12 are detailed in other scenarios (exception and sub-scenario) like "Register 
Customer", "Local Supplier bid for order", "International Supplier bid for order" and "Process Bids". It means that Petri-Nets should be generated for referenced scenarios (Register Customer-T1.1, Local Supplier bid for order-T10, International Supplier bid for order-T11 and Process Bids-T12) and replaced into the main Petri-Net of "Submit Order".

Figure 28 (a) and (c) show the Petri-Nets derived for like "Register Customer" and "Process Bids" scenarios, and where must be substituted in "Submit Order" Main Petri-Net. In Figure 28 (b), transitions T10 and T11 reference the "Local Supplier bid for order" and "International Supplier bid for order" scenarios; they are executed in a non-sequential order and must be replaced in the corresponding transitions.

Figure 29 shows the Integrated Petri-Net of "Submit Order" scenario. The sequentially related scenarios (T1.1. REGISTER CUSTOMER and T12. PROCESS BIDS) are substituted by the Petri-Nets depicted in Figure 28 (a) and (c). 


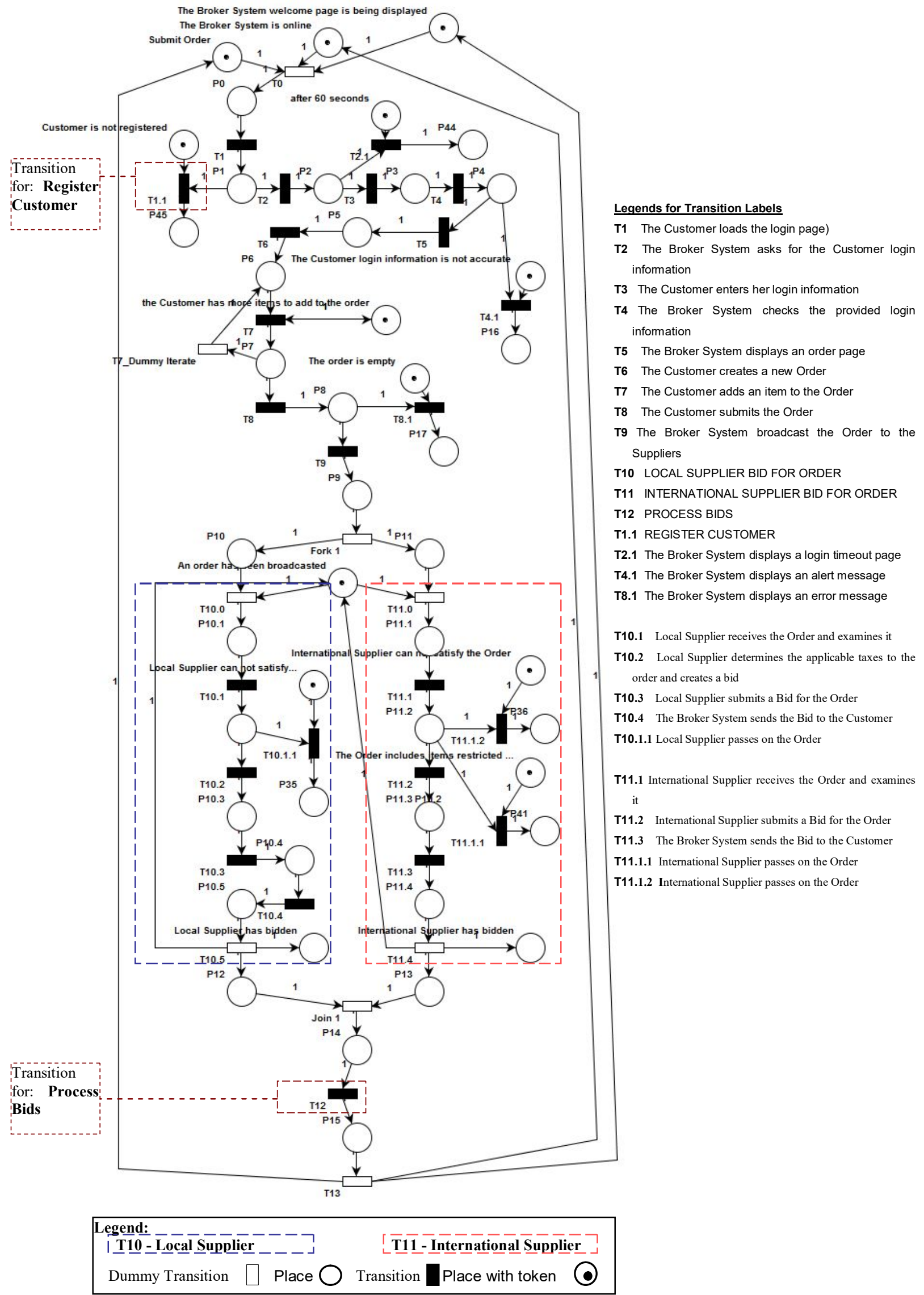

Figure 29 - Integrated Petri-Net of "Submit Order". 


\subsection{4. \\ Preservation of Properties}

We believe that Petri-Net derived from a scenario (Method 1 - Figure 26) preserves the event sequences and conditions described within a scenario, as we explain next.

Demonstration 1: A scenario describes situations (Leite et al., 2000) in the form of episodes or exceptions. From a given initial state (context with all necessary resources, pre-conditions and constraints), the execution of an episode or the treatment of an exception leads into another state. According to the transformation method (Method 1 - Figure 26), the execution of a episode or the treatment of a exception is denoted by the firing of a Petri-Net transition, which changes a marking $\boldsymbol{M}$ (source state) to $\boldsymbol{M}^{\prime}$ (target state). Each transition is labeled with the sentence or solution performed by an episode or exception, respectively. For each transition translated from an episode or exception: (1) Input places are created to denote the locations of its pre-conditions, causes and constraints; (2) Output places are created to denote the location of its post-conditions. Therefore, the execution of scenario episodes or exceptions is modeled by firing a sequence of Petri-Net transitions.

Moreover, the properties of the Petri-Nets derived from related scenarios are preserved when they are synthesized into a whole Petri-Net, because the synthesis procedure (Method 2 - Figure 27) does not introduce new non-deterministic situations (Non-determinism is the main source of synchronization defects), as we explain next.

Demonstration 2: A Petri-Net is the formal representation of a scenario. We integrate the related Petri-Nets in order to obtain a partial initial system design. The integrated Petri-Net reflects exactly the original properties of the synthesized Petri-Nets. Among the synthesized Petri-Nets, there are common places (with the same labels) that denote the same conditions or states, and there are places or transitions that reference (in their labels) other Petri-Nets. Our integration method (Method 2 - Figure 27) is basically the fusion of common places and the substitution of places or transitions by the corresponding Petri-Nets: (1) the substitution (Definition 6.3, 6.4 and 6.5) of places or transitions by sequentially related Petri-Nets do not create any new arcs between these Petri-Nets, i.e. the 
substitution of places or transitions is done by fusing with the first "input dummy place (Start)" or the last "output dummy place" (Finish) of the replacing PetriNet; and (2) the fusion (Definition 6.6) of concurrently related Petri-Nets does not create any new arcs between these Petri-Nets, i.e. the fusion of places is done by fusing common places.

Figure 30 illustrates the application of substitution input place and concurrent fusion place operations to obtain two integrated Petri-Nets: (a) First example, a constraint of a scenario $\mathbf{S 1}$ is detailed in other scenario $\mathbf{S 2}$ (sequential relationship); and (b) Second example, two scenarios S1 and S2 interact concurrently because the post-condition of the first one $\mathbf{S 1}$ has the same label that the pre-condition of the second one $\mathbf{S 2}$.

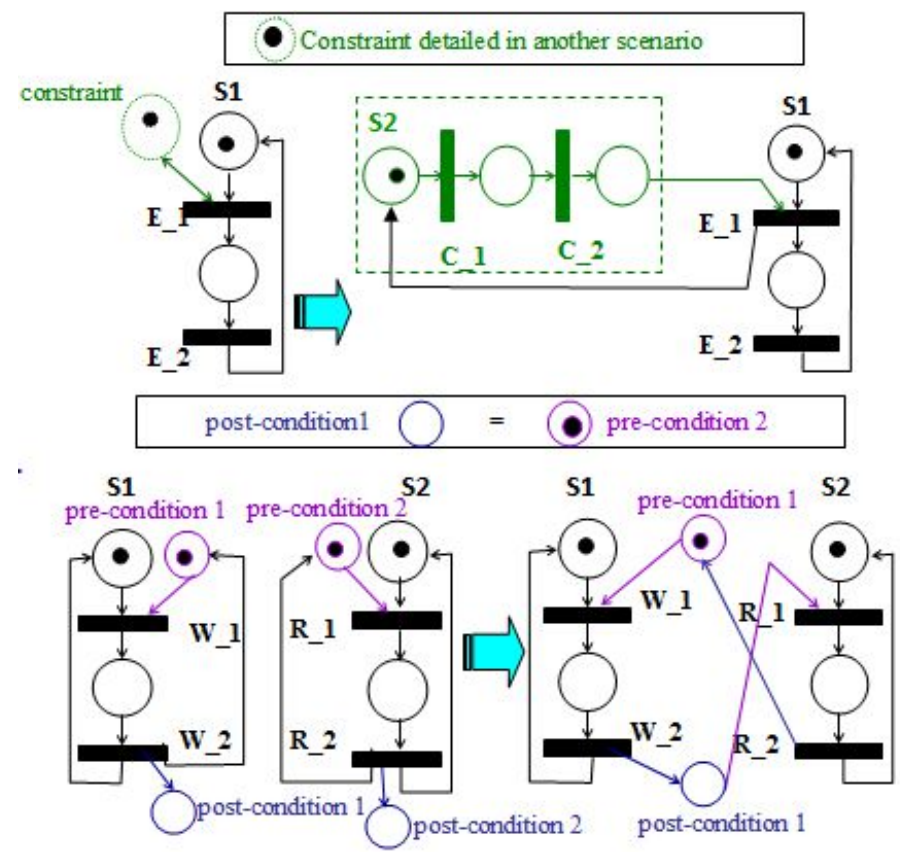

Figure 30 - Substitution input place (a) and concurrent fusion place (b).

\section{4.}

\section{Analyzing Scenarios}

The process of analysis involves checking some structural and behavioral properties in Scenario descriptions and equivalent Petri-Nets, respectively. The analysis of these properties can be addressed through the use of static and dynamic analysis techniques, or the combination of them.

In order to detect defects related to Unambiguity and Completeness, our analysis approach performs a static analysis. Unambiguity analysis detects ambiguous terms or phrases within internal scenario sentences. Completeness analysis checks the style and content of internal scenario elements, and detects 
defects in the relationships among related scenarios. Some tasks related to these activities can be supported by NLP techniques.

In order to detect defects related to behavioral properties, our analysis approach performs a dynamic analysis. The analysis of the behavior of a set of scenarios can detect inconsistency or incorrectness indicators, such as deadlock situations. Consistency analysis detects some defects due to non-determinism and synchronization issues, we have made use of Place-Transition Petri-Nets (Murata, 1989) for analysis of: (1) static properties like Correct Token Passing and Fully Connected (related to Feasibility); and (2) dynamic properties like Determinism, Boundedness, Reversibility and Deadlock free (related to Non-interferential, Boundedness, Reversibility and Liveness).

A Quality Model for Scenarios and heuristics to detect defect indicators that hurt Unambiguity, Completeness and Consistency in scenarios is presented in Chapter 3. Below we detail the steps for Scenarios analysis.

\subsection{1.}

\section{Unambiguity Analysis}

Natural language plays an important role in scenario specifications because scenario elements are described using NL. Due to the inherent ambiguity, the use of NL is a critical issue. NLP techniques can be used for the linguistic analysis of NL scenario descriptions and search defect indicators that hurt Unambiguity. These indicators can be grouped in categories (Vagueness, Subjectiveness, Optionality, Multiplicity, Quantifiability, Readabiity, Minimality, Weakness and Implicitly) and detected by lexical analysis. Readabiity and Minimality contributes positively to Unambiguity.

To evaluate Readability, we use the Coleman-Liau Formula readability metric. This metric is based on the number of the letters, words and sentences of a requirement (Wilson et al., 1997).

Coleman-Liau Formula readability metric: (5.89* letters/words$0.3 *$ sentences/(100*words)-15.8]). The reference value of this formula for an easy-to-read technical document is 27.60 , if it is $<17.10$ and $>55.80$ the document is difficult-to-read.

In scenario, the evaluation of properties related to unambiguity is performed by reading the different scenario elements (typically involving events: title, goal, 
episodes and exceptions), and searching for defect indicators (stored in indicators dictionaries) that contribute (positively or negatively) to Unambiguity: Vagueness, Subjectiveness, Optionality, Multiplicity, Quantifiability, Minimality, Weakness and Implicitly (See Table 7, Chapter 3).

A summary of unambiguity evaluation of Scenario elements is outlined below in Method 3 (Figure 31). The detected defects are classified as Warning (See Section 4.5).

Method 3: Analyze Unambiguity

Input: Scenario $S=$ (Title, Goal, Context, Resource, Actor, Episodes, Exception);

Ambiguous Indicators A = \{Vaqueness, Subjectiveness, Optionality, Multiplicity, Quantifiability, Weakness, Implicitly $\}$;

Output: Feedback F = (Informations, Warnings, Errors)

Begin:

1. Evaluate Readability index:

$\rightarrow$ IF Readability Index of Title $>55.8$ THEN Add "Readability: Title is difficult-to-read" to W

$\rightarrow$ For every Episode in episodes of Scenario $\mathrm{S}$

$\rightarrow$ IF Readability Index of Sentence > 55.8 THEN Add "Readability: Episode is difficult-to-read" to W;

$\rightarrow$ For every Exception in exceptions of Scenario $\mathbf{S}$ :

$\rightarrow$ IF Readability Index of Solution > 55.8 THEN Add "Readability: Exception is difficult-to-read" to W;

2. Evaluate Minimality:

$\rightarrow$ IF Title contains a Text after a not minimal term THEN Add "Minimality: Title describes an ambiguous situation" to W:

$\rightarrow$ For every Episode in episodes of Scenario S:

$\rightarrow$ IF Sentence contains a Text after a not minimal term THEN Add "Minimality: Episode describes a non-minimal sentence" to W;

$\rightarrow$ For every Exception in exceptions of Scenario S

$\rightarrow$ IF Solution contains a Text after a not minimal term THEN Add "Minimality: Exception describes a non-minimal solution" to W;

3. Evaluate Vagueness:

$\rightarrow$ IF Title contains a Vague term THEN Add "Vagueness: Title describes an ambiguous situation" to W

$\rightarrow$ For every Episode in episodes of Scenario $\mathbf{S}$ :

$\rightarrow$ IF Sentence contains a Vague term THEN Add "Vagueness: Episode describes an ambiguous sentence" to W;

$\rightarrow$ For every Exception in exceptions of Scenario $S$

$\rightarrow$ IF Solution contains a Vague term THEN Add "Vagueness: Exception describes an ambiguous solution" to W;

4. Evaluate Subjectiveness:

$\rightarrow$ IF Title contains a Subjective term THEN Add "Subjectiveness: Title describes an ambiguous situation" to W:

$\rightarrow$ For every Episode in episodes of Scenario S:

$\rightarrow$ IF Sentence contains a Subjective term THEN Add "Subjectiveness: Episode describes an ambiguous sentence" to W;

$\rightarrow$ For every Exception in exceptions of Scenario S

$\rightarrow$ IF Solution contains a Subjective term THEN Add "Subjectiveness: Exception describes an ambiguous solution" to W;

5. Evaluate Optionality:

$\rightarrow$ IF Title contains a Optional term THEN Add "Optionality: Title describes an ambiguous situation" to W:

$\rightarrow$ For every Episode in episodes of Scenario S:

$\rightarrow$ IF Sentence contains a Optional term THEN Add "Optionality: Episode describes an ambiguous sentence" to W:

$\rightarrow$ For every Exception in exceptions of Scenario S

$\rightarrow$ IF Solution contains a Optional term THEN Add "Optionality: Exception describes an ambiguous solution" to W;

6. Evaluate Multiplicity:

$\rightarrow$ IF Title contains a Multiple term THEN Add "Multiplicity: Title describes an ambiguous situation" to W;

$\rightarrow$ For every Episode in episodes of Scenario S:

$\rightarrow$ IF Sentence contains a Multiple term THEN Add "Multiplicity: Episode describes an ambiguous sentence" to W:

$\rightarrow$ For every Exception in exceptions of Scenario $\mathbf{S}$

$\rightarrow$ IF Solution contains a Multiple term THEN Add "Multiplicity: Exception describes an ambiguous solution" to W;

7. Evaluate Quantifiability:

$\rightarrow$ IF Title contains a Quantifiable term THEN Add "Quantifiability: Title describes an ambiguous situation" to W

$\rightarrow$ For every Episode in episodes of Scenario S:

$\rightarrow$ IF Sentence contains a Quantifiable term THEN Add "Quantifiability: Episode describes an ambiguous sentence" to W;

$\rightarrow$ For every Exception in exceptions of Scenario $\mathbf{S}$

$\rightarrow$ IF Solution contains a Quantifiable term THEN Add "Quantifiability: Exception describes an ambiguous solution" to W;

8. Evaluate Weakness:

$\rightarrow$ IF Title contains a Weak term THEN Add "Weakness: Title describes an ambiguous situation" to W;

$\rightarrow$ For every Episode in episodes of Scenario S:

$\rightarrow$ IF Sentence contains a Weak term THEN Add "Weakness: Episode describes an ambiguous sentence" to W;

$\rightarrow$ For every Exception in exceptions of Scenario S:

$\rightarrow$ IF Solution contains a Weak term THEN Add "Weakness: Exception describes an ambiguous solution" to W;

9. Evaluate Implicitly:

$\rightarrow$ IF Title contains an Implicit term THEN Add "Implicitly: Title describes an ambiguous situation" to W

$\rightarrow$ For every Episode in episodes of Scenario S:

$\rightarrow$ IF Sentence contains an Implicit term THEN Add "Implicitly: Episode describes an ambiguous sentence" to W

$\rightarrow$ For every Exception in exceptions of Scenario S

$\rightarrow$ IF Solution contains a Implicit term THEN Add "Implicitly: Exception describes an ambiguous solution" to W; End 10. Return Feedback $\mathbf{F}=\{\mathbf{W}\}$;

Figure 31 - Unambiguity Analysis (Method 3). 
Following are examples of Unambiguity defects pointed out by our analysis approach in the "Online Broker System"; the underlined words or phrases are the indicators detected by our approach to point out the episode sentence containing the defect:

- Submit Order scenario: Episode 1. The Customer enters her login information (Implicitly);

- Submit Order scenario: Episode 4. The Broker System checks the provided login information (Vagueness);

- Local Supplier scenario: Episode 3. Local Supplier receives the Order and examines it (Multiplicity);

- Handle Payment: Episode 2. The Customer provides her Credit Card information (Implicitly).

\subsection{2.}

\section{Completeness Analysis}

To evaluate Completeness, we detect missing information in internal (intrascenario) and external aspects (inter-scenario) of scenarios. The intra-scenario properties include: Atomicity, Simplicity, Uniformity, Usefulness and Conceptually Soundness. The inter-scenario properties include: Integrity, Coherency and Uniqueness. Other important property related to completeness is Feasibility (See Chapter 3).

Thus, the syntax and semantic of each element in scenario and its relationships must be described as established in the scenario model and grammar (Table 13).

The violation of properties related to completeness is detected by traversing every scenario element (Title, Goal, Context, Resource, Actor, Episodes, Exception), and following the checklist with verification heuristics described in Chapter 3 (Table 8, Table 9, Table 10 and Table 11). For each heuristic, we defined a set of common defect indicators.

In order to search defect indicators that hurt Completeness properties, we classify the defects detection heuristics according to the analysis strategy used by them, i.e., some of the heuristics verify that every scenario element contains its relevant components (Lexical), others verify that every scenario elements and its 
internal components follows the grammar rules (Syntactic). In Chapter 5 is detailed the classification of each one of the defects detection heuristics.

\subsubsection{1.}

\section{Lexical Analysis}

To detect lexical defects that hurt Uniformity it is enough to verify the conformance of scenario elements to the scenario model. Figure 32 illustrates examples of lexical analysis of an episode (a) and an exception (a) described in the "Online Broker System" scenarios.

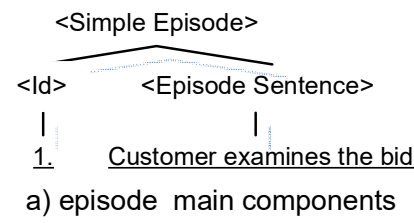

Figure 32 - Lexical analysis of simple episode (a) and exception (b) elements.

To detect lexical defects that hurt Atomicity, Simplicity, Usefulness, Conceptually Soundness, Integrity and Coherency, it is enough to search for multiplicity indicators in the scenario title, to count the number of episodes in each scenario, to check that every actor or resource is used in episodes, to verify the presence of Linking-verb or State-Verb in conditions (pre-condition, postcondition, episode condition and exception cause), to verify the existence of referenced scenarios or pre-conditions, and to check the coherency between related scenario pre-conditions (geographical location and temporal location), respectively.

Following are examples of Completeness lexical defects pointed out by our analysis approach in the "Online Broker System"; the underlined words or phrases are the indicators detected by our approach to point out the sentence containing the defect:

- International Supplier bid for order: Exception 1.1 IF The Order includes items restricted for exportation THEN International Supplier passes on the Order (Soundness - Missing Linking-Verb or State-Verb);

- Submit Order: Num. episodes > 10 (Usefulness - Too long scenario);

- Submit Order: Context Pre-condition - The Broker System is online (Integrity - It is an uncontrollable fact does not satisfied by a Postcondition of other scenario); 
- Process Bids: Actor - Broker System (Usefulness - never participates in episodes).

\subsubsection{2. \\ Syntactical Analysis}

To detect syntactic defects that hurt Atomicity, Simplicity, Usefulness, Conceptually Soundness and Uniqueness, it is necessary to check that every sentence contains significant information like the main Verb, Direct Object modified, and optionally the Subject and Indirect Objects. Considering that a scenario sentence (typically involving events: title, episode sentence and exception solution) performs an action (Action-Verb) that can use or modify resources (Objects) and be executed by actors (Subjects), there are three basic types of structured sentences: 1) verb-object (for writing the scenario title or reference another scenario), 2) subject-verb-object, and 3) subject-verb-objectindirect-object (for writing episode sentences or exception solutions).

Phrase-structure parsing or dependency parsing (Stanford, 2015) strategies can be used to identify the significant information of scenario sentences. The result of the parsing is a parse tree, in which the sentence is parsed into the Subject or Object of a Verb; then the non-leaf nodes are Part-of-Speech (Klein and Manning, 2003) tags where "NN" and "VB" represents the noun phrase and verb phrase respectively; the leaf nodes are tokenized words of the original textual sentence. Stanford (2015) tool is a program that could be used to analyze the grammatical structure of sentences.

Figure 33 illustrates the parse tree for sentences described in the "Online Broker System" scenarios.

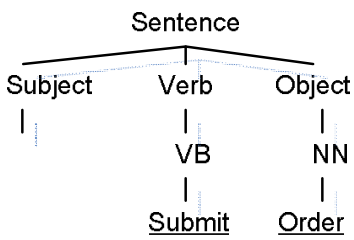

a) verb-object

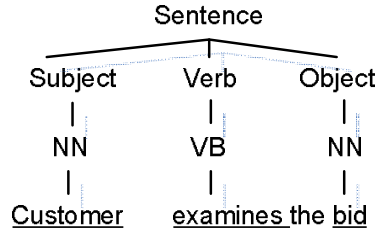

b) subject-verb-object

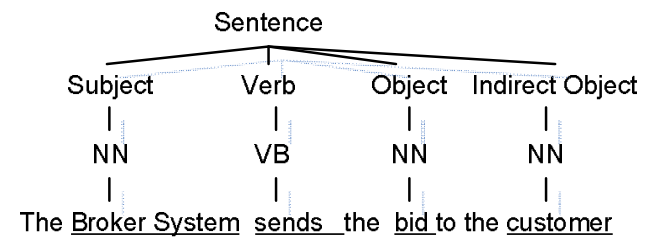

c) subject-verb-object-indirect object

Figure 33 - Parse tree for verb-object (a), subject-verb-object (b) and subject-verbobject-indirect-object (c) sentences.

Following are examples of Completeness syntactic defects pointed out by our analysis approach in the "Online Broker System"; the underlined words or 
phrases are the indicators detected by our approach to point out the sentence containing the defect:

- Local Supplier bid for order: Episode 1. Local Supplier receives the Order and examines it (Simplicity - Contains more than one Action-Verb);

- International Supplier bid for order: Episode 3. The Broker System sends the Bid to the Customer (Usefulness - Actor/Resource mentioned in episode is not included in the Actor/Resource element);

- Process Bids: Episode 4. System put an order with the selected bidder (Simplicity - Missing Action-Verb in Present Tense form);

- Local Supplier bid for order: "Local Supplier bid for order" and "International Supplier bid for order" (Uniqueness - They are potentially duplicated! Their Titles share the same Action-Verb and direct Object).

Method 4: Analyze Completeness

Input: Scenario $S=$ (Title, Goal, Context, Resource, Actor, Episodes, Exception); Completeness Defect Indicators C $=$ \{Atomicity, Simplicity, Uniformity, Usefulness, Conceptually Soundness, Integrity, Coherency, Uniqueness, Feasibility\};

Output: Feedback F = (Informations, Warnings, Errors)

Begin:

1. IF Title describes multiple situations THEN Add "Atomicity: Title must express only a situation" to $\mathbf{W}$;

2. IF Goal describes multiple situations THEN Add "Atomicity: Goal must express only a situation" to W:

3. For each Scenario Element in Scenario $\mathbf{S}$ :

$\rightarrow$ IF Scenario Element does not follow the scenario model THEN Add "Uniformity: Scenario Element must follow the scenario model" to $\mathbf{E}$;

4. For every Episode in episodes of Scenario S:

$\rightarrow$ IF Episode is not Readable THEN Add "Simplicity: Episode is difficult-to-read" to W;

$\rightarrow$ IF Episode is not consistent with Actors and Resources THEN Add "Usefulness: Episode must be consistent with actors and resources" to $\mathbf{W}$;

$\rightarrow$ IF Episode does not perform actions and change states THEN Add "Conceptually Soundness: Episode should perform actions and change states" to W;

5. For every Exception in exceptions of Scenario S:

$\rightarrow$ IF Exception is not Readable THEN Add "Simplicity: Exception is difficult-to-read" to W;

$\rightarrow$ IF Exception is not consistent with episodes THEN Add "Usefulness: Exception must be consistent with branching episode" to W;

$\rightarrow$ IF Exception does not perform actions and change states THEN Add "Conceptually Soundness: Exception should perform actions and change states" to W;

6. Identify sequential relationships of the Scenario $\mathbf{S}$ by Pre-condition. Post-condition, Constraint, Sub-scenario or Exception;

7. For every Related Scenario in Sequentially Scenarios:

$\rightarrow$ IF Related Scenario does not exist in the set of scenarios THEN Add "Integrity: Related Scenario should exist in the set of scenarios" to $\mathbf{E}$;

$\rightarrow$ IF Related Scenario does not use a common terminology with the Scenario S THEN Add "Coherency: Related Scenario should use a common terminology, e.g. pre-conditions, temporal location and geographical location should be coherent with the main Scenario" to I:

8. Get the set of Scenarios of the Project;

9. For every Scenario SS in the set of Scenarios of the Project:

$\rightarrow$ IF Scenario SS is duplicated of Scenario S THEN Add "Uniqueness: scenarios should not share the same Title, Goal or Episodes" to W;

10. Derive Petri-Net PN from Scenario $\mathbf{S}$ (Method 1);

11. IF there are places or transitions that do not interact with others in PN THEN Add "Feasibility: Petri-Net contains

isolated sub nets" to $\mathrm{E}$;

End

2. Return Feedback $\mathbf{F}$

Figure 34 - Completeness Analysis (Method 4). 
A summary of Completeness evaluation of a Scenario and its related scenarios is outlined in Method 4 (Figure 34). The detected defects are classified as Information, Warning or Error (See Section 4.5).

\subsection{3.}

\section{Consistency Analysis}

According to Denger et al (2005), the most difficult defects to detect by static analysis are consistency defects; these defects can be detected with much effort using reading or inspection techniques. In order to address this issue, we integrated the dynamic analysis.

Dynamic analysis of scenarios can be performed by rigorous analysis techniques, i.e., from a main scenario, a set of related scenarios (sequentially and non-sequentially related) are identified and translated into executable models (Petri-Nets), which are executed in a formal analysis environment like PIPE2 (2015).

To evaluate Consistency, we integrate the Petri-Nets corresponding to related scenarios into the Petri-Net derived from a main scenario, and detect wrong information in the Integrated Petri-Net. The consistency related properties include: Non-interferential, Boundedness, Reversibility and Liveness (See Chapter 3).

The violation of properties related to consistency can be detected by generating the reachability graph of the equivalent Integrated Petri-Net, and analyzing this graph following the checklist with verification heuristics described in Chapter 3 (Table 12). For each heuristic, we identified a set of common defect indicators.

\subsubsection{1.}

\section{Managing the State Explosion}

State explosion issue is a serious problem when applying Petri-Net analysis to large systems. A contribution of this thesis is a MULTI-STEP consistency analysis method to manage this problem. The reachability analysis of an Integrated Petri-Net can be performed in a compositional way, where: (1) PetriNets corresponding to sequentially related scenarios are removed from the Integrated Petri-Net, (2) Petri-Nets corresponding to non-sequentially related 
scenarios are preserved into the Integrated Petri-Net because they might interact among them, and (3) the resulting Petri-Nets are analyzed separately.

In this method, the Integrated Petri-Net is divided into a set of Petri-Nets (Petri-Nets corresponding to sequentially related scenarios and the Integrated Petri-Net) that preserves the properties and concurrency characteristics of the Integrated Petri-Net.

It is possible because the process to obtain an Integrated Petri-Net from a main scenario and its relationships does not introduce new arcs when a Petri-Net corresponding to a related scenario is fused or substituted into a place or transition of the Integrated Petri-Net (Method 2 - Figure 27, Section 4.3.2).

A summary of the MULTI-STEP Consistency evaluation of an equivalent Petri-Net is outlined below in Method 5 (Figure 35). The detected defects are classified as Information, Warning or Error (See Section 4.5).

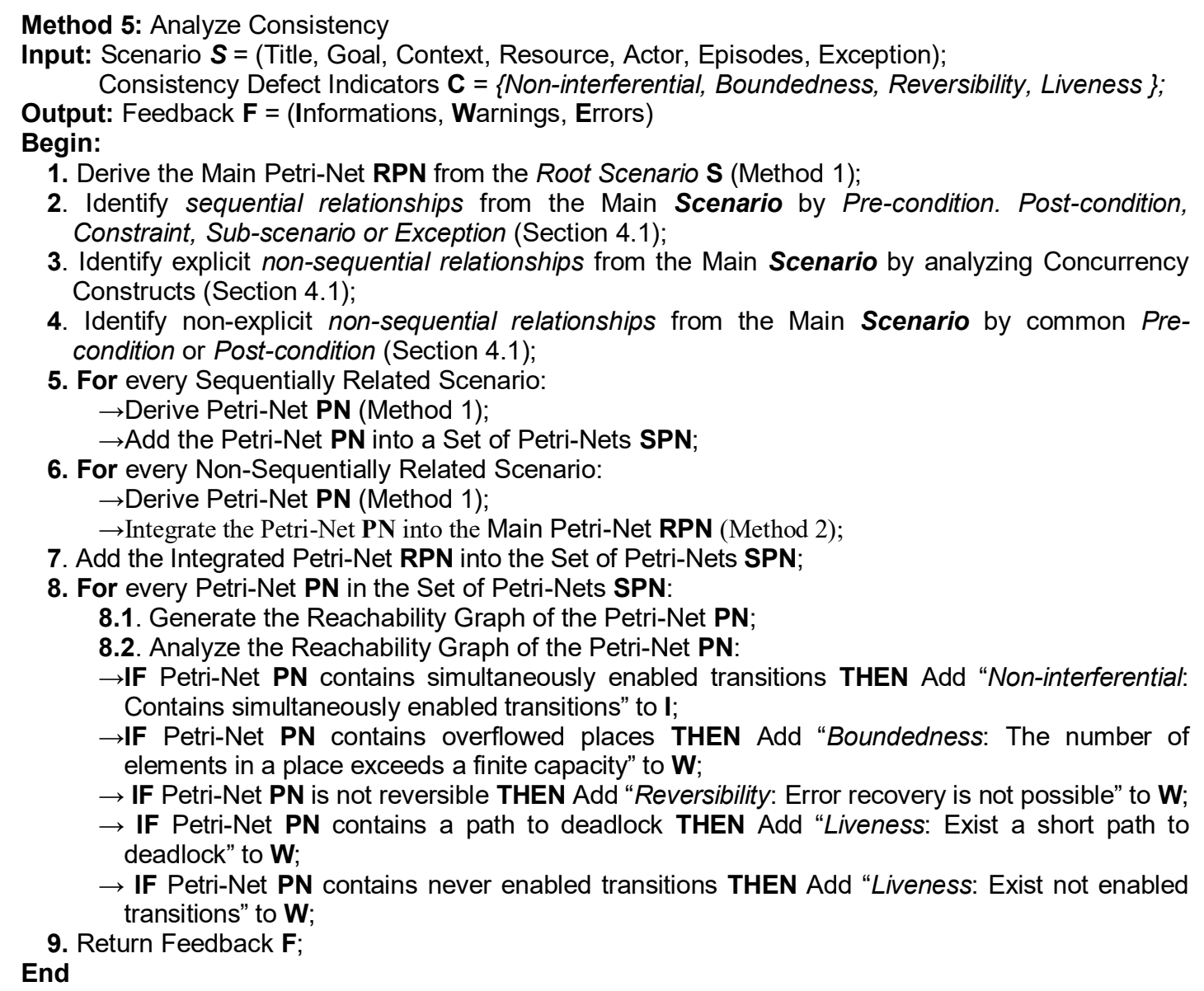

Figure 35 - Consistency Analysis (Methoid 5).

This MULTI-STEP method reduces the state explosion problem by: (1) increasing the feasibility of Petri-Nets and (2) enabling the verification of 
properties which may fail on Integrated Petri-Net due to a state explosion problem.

In the "Online Broker System", the main scenario is the "Submit Order" scenario, and the Suppliers' scenarios are executed concurrently (non-sequential), as shown in Figure 22 and Figure 23.

Figure 36 depicts how the Petri-Nets derived from non-sequentially related scenarios (LOCAL SUPPLIER BID FOR ORDER and INTERNATIONAL SUPPLIER BID FOR ORDER) are substituted into the corresponding transitions (T10 and T11) of the Petri-Net derived from the "Submit Order" scenario. The Petri-Nets corresponding to REGISTER CUSTOMER and PROCESS BIDS transitions (T1.1 and T12) are not integrated because they are sequentially related to "Submit Order" scenario, and they can be analyzed separately because they do not interact concurrently with the main scenario (avoiding the state explosion issue).

From the Integrated Petri-Net in Figure 36, we: a) generate the reachability graph, and b) apply the reachability analysis technique to detect consistency defects. Figure 37 depicts the reachability graph and results of the reachability analysis for "Submit Order" scenario using the PIPE2 (2015). Nodes are reachable states; arcs are transitions performed to reach a state, and $\boldsymbol{S}_{\boldsymbol{0}}$ is the initial state.

The reachability analysis of the integrated Petri-Net PN of "Submit Order" scenario (Figure 22 and Figure 23) using the PIPE2 (2015) tool pointed out the following Consistency defects in the "Online Broker System":

- Non bounded because it presents overflowed places (Local Supplier has bidden, International Supplier has bidden);

- Non live because the firing sequence $\langle($ SUBMIT ORDER) T0 $\rightarrow$ T1 $\rightarrow$ T2 $\rightarrow \mathrm{T} 3 \rightarrow \mathrm{T} 4 \rightarrow \mathrm{T} 5 \rightarrow \mathrm{T} 6 \rightarrow \mathrm{T} 7 \rightarrow \mathrm{T} 8 \rightarrow \mathrm{T} 9 \rightarrow$ Fork $1 \rightarrow($ LOCAL SUPPLIER BID FOR ORDER) T10.0 $\rightarrow$ T10.1 $\rightarrow$ T10.2 $\rightarrow$ T10.3 $\rightarrow$ T10.4 $\rightarrow$ T10.5 $\rightarrow$ ( INTERNATIONAL SUPPLIER BID FOR ORDER) $\mathrm{T} 11.0 \rightarrow \mathrm{T} 11.1 \rightarrow \mathrm{T} 11.2 \rightarrow \mathrm{T} 11.3 \rightarrow \mathrm{T} 11.4 \rightarrow$ (SUBMIT ORDER) Join_1 $\rightarrow \mathrm{T} 12 \rightarrow \mathrm{T} 12 \rightarrow \mathrm{T} 13 \rightarrow \mathrm{T} 0 \rightarrow \mathrm{T} 1 \rightarrow \mathrm{T} 2 \rightarrow \mathrm{T} 3 \rightarrow \mathrm{T} 4 \rightarrow \mathrm{T} 5 \rightarrow \mathrm{T} 6$ $\rightarrow \mathrm{T} 7 \rightarrow \mathrm{T} 8 \rightarrow \mathrm{T} 9 \rightarrow$ Fork_1 $\rightarrow$ ( LOCAL SUPPLIER BID FOR ORDER) $\mathrm{T} 10.0 \rightarrow \mathrm{T} 10.1 \rightarrow \mathrm{T} 10.2 \rightarrow \mathrm{T} 10.3 \rightarrow \mathrm{T} 10.4 \rightarrow \mathrm{T} 10.5 \rightarrow($ INTERNATIONAL SUPPLIER BID FOR ORDER) T11.0 $\rightarrow$ T11.1 $\rightarrow$

T11.1.1 $>$ is a shortest path to Deadlock; 
- Non reversible, because it is not bounded, not safe and not live. There is a deadlock when the "The Order includes items restricted for exportation" in the "International Supplier Bid for Order" scenario.

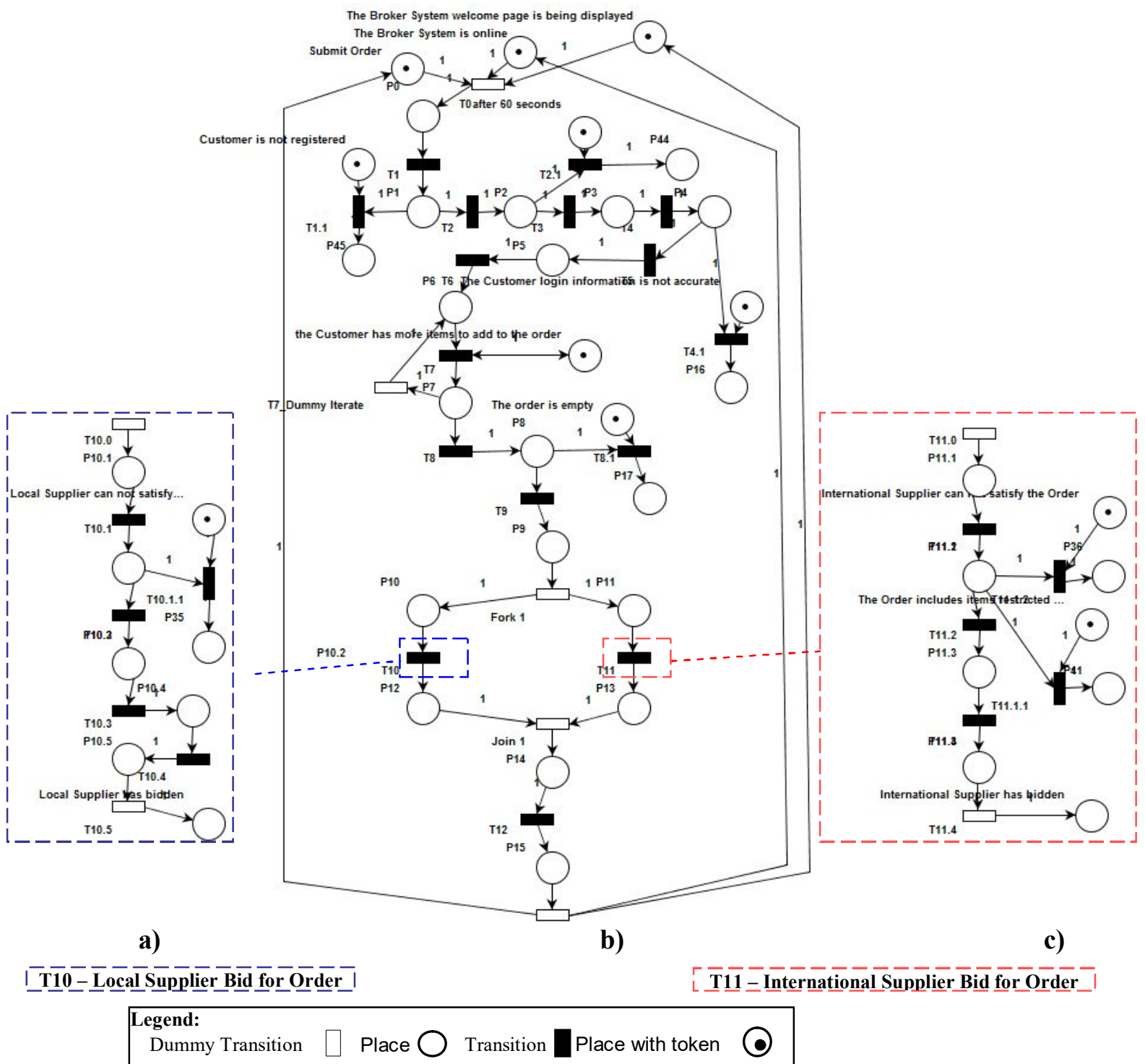

Legends for Transition Labels

T10.1 Local Supplier receives the Order and examines it

T10.2 Local Supplier determines the applicable taxes to the order and creates a bid

T10.3 Local Supplier submits a Bid for the Order

T10.4 The Broker System sends the Bid to the Customer

T10.1.1 Local Supplier passes on the Order

T11.1 International Supplier receives the Order and examines it

T11.2 International Supplier submits a Bid for the Order

T11.3 The Broker System sends the Bid to the Customer

T11.1.1 International Supplier passes on the Order

T11.1.2 International Supplier passes on the Order

$\mathrm{P}<\mathrm{x}><\mathrm{y}>$ are input or output dummy places

T8.1 The Broker System displays an error message

Figure 36 - Integrating "Suppliers" Petri-Nets into the Petri-Net of "Submit Order". 


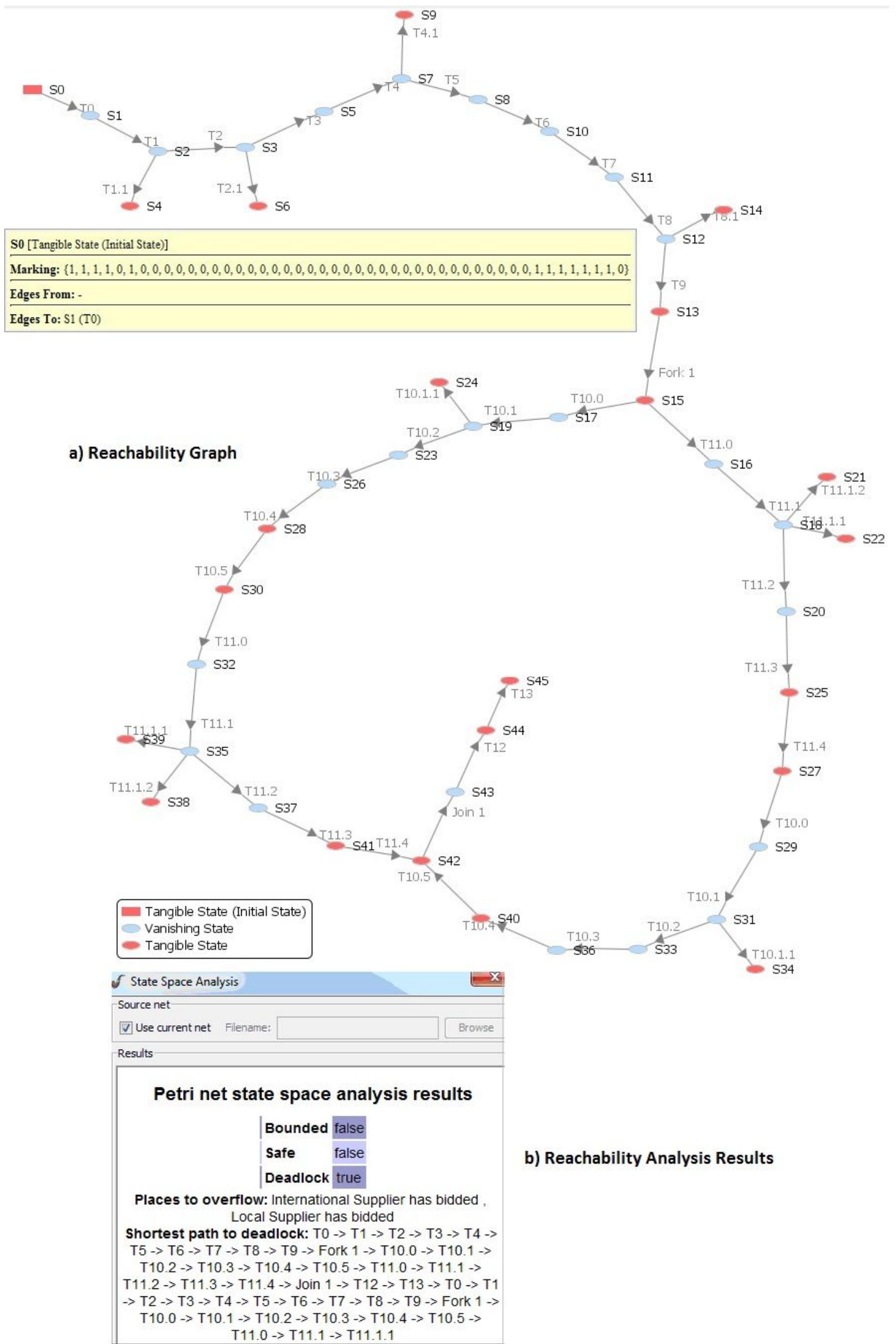

Figure 37 - Reachability graph (a) and Reachability analysis results (b) of "Submit Order" scenario. 


\subsection{4. \\ Correctness Analysis}

Correctness is the main quality in scenarios, and it is difficult to evaluate and achieve because it depends on semantic analysis of scenarios and user's satisfaction. To address some issues related to the correctness of scenarios, we could formalize the scenario descriptions using formal methods or analyze semantically the information contained in sentences described within scenarios.

Formal methods are a powerful means to evaluate scenarios because they provide a theoretical framework in which erroneous situations could be predicted. However, specific skills are needed, and this increases their application cost.

The use of NLP techniques could help in identifying the syntactic information of sentences, i.e. NLP techniques can identify Part-of-Speech (POS) tags like "Nouns" and "Verbs" in textual sentences. However, it is impossible to detect semantic defects with high precision (Lucassen et al., 2015).

In this work, NLP techniques (syntactic analysis) and Formal methods (Petri-Nets) are used for detecting defects that hurt Completeness and Consistency qualities, respectively. The use of these techniques can contribute positively to the Correctness of scenarios.

In our analysis approach, we introduced a novel perception of correctness and its complex relationships with unambiguity, completeness and consistency describing it as a quality that should be satisficed by contributions of related qualities or properties (See Chapter 3).

\section{5.}

\section{Generating Feedback}

By combining static and dynamic analysis techniques, we are able to detect defects that hurt the properties related to unambiguity, completeness and consistency qualities, and, consequently address the defects that hurt Correctness of scenario-based specifications.

In Table 20, we summarize how the defects are detected and classified by our analysis approach (Method 3,4 and 5). These defects are detected by heuristics that implement Lexical, Syntactical or Reachability analysis strategies and classified as: Information, Warning or Error. Implementation details of these heuristics are presented in Chapter 5 (Section 5.3.5). Information reveals that the 
requirements engineer may have forgotten to specify some information related to a scenario element. Warning reveals that the requirements engineer may have introduced some confusing information or forgotten to inform and important scenario element. Error reveals that the requirements engineer may have introduced wrong information related to a scenario element.

Table 20 - Scenario Defects Classification

\begin{tabular}{|c|c|c|c|c|}
\hline Quality & Property & Heuristic & Analysis Strategy & Defect Category \\
\hline \multirow[t]{8}{*}{ Unambiguity } & Vagueness & 1 & Lexical & Warning \\
\hline & Subjectiveness & 1 & Lexical & Warning \\
\hline & Optionality & 1 & Lexical & Warning \\
\hline & Weakness & 1 & Lexical & Warning \\
\hline & Multiplicity & 1 & Lexical & Warning \\
\hline & Implicitly & 1 & Lexical & Warning \\
\hline & Quantifiability & 1 & Lexical & Warning \\
\hline & Readability & 1 & Lexical & Warning \\
\hline \multirow[t]{38}{*}{ Completeness } & \multirow[t]{3}{*}{ Atomicity } & 1 & Lexical & Warning \\
\hline & & 2 & Lexical & Warning \\
\hline & & 3 & Syntactic & Warning \\
\hline & \multirow[t]{6}{*}{ Simplicity } & 1 & Syntactic & Warning \\
\hline & & 2 & Syntactic & Warning \\
\hline & & 3 & Lexical & Information \\
\hline & & 4 & Lexical & Warning \\
\hline & & 5 & Lexical & Warning \\
\hline & & 6 & Lexical & Warning \\
\hline & Uniformity & 1 & Lexical & Warning \\
\hline & \multirow[t]{6}{*}{ Usefulness } & 1 & Lexical & Warning \\
\hline & & 2 & Syntactic & Warning \\
\hline & & 3 & Lexical & Warning \\
\hline & & 4 & Syntactic & Warning \\
\hline & & 5 & Lexical & Warning \\
\hline & & 6 & Lexical & Warning \\
\hline & \multirow[t]{9}{*}{ Conceptually Soundness } & 1 & Syntactic & Warning \\
\hline & & 2 & Semantic & Warning \\
\hline & & 3 & Semantic & Warning \\
\hline & & 4 & Syntactic & Warning \\
\hline & & 5 & Lexical & Information \\
\hline & & 6 & Lexical & Information \\
\hline & & 7 & Lexical & Information \\
\hline & & 8 & Syntactic & Warning \\
\hline & & 9 & Lexical & Information \\
\hline & \multirow[t]{3}{*}{ Integrity } & 1 & Lexical & Error \\
\hline & & 2 & Lexical & Information \\
\hline & & 3 & Lexical & Information \\
\hline & \multirow[t]{3}{*}{ Coherency } & 1 & Semantic & Warning \\
\hline & & 2 & Lexical & Warning \\
\hline & & 3 & Lexical & Warning \\
\hline & \multirow[t]{5}{*}{ Uniqueness } & 1 & Lexical & Warning \\
\hline & & 2 & Lexical & Warning \\
\hline & & 3 & Lexical & Warning \\
\hline & & 4 & Lexical & Warning \\
\hline & & 5 & Syntactic & Warning \\
\hline & \multirow[t]{2}{*}{ Feasibility } & 1 & Lexical & Error \\
\hline & & 2 & Lexical & Error \\
\hline \multirow[t]{4}{*}{ Consisyency } & Non-interferential & 1 & Reachability analysis & Information \\
\hline & Boundedness & 1 & Reachability analysis & Warning \\
\hline & Reversibility & 1 & Reachability analysis & Warning \\
\hline & Liveness & 1 & Reachability analysis & Warning \\
\hline
\end{tabular}


The presence of defects classified as Information, Warning or Error is likely, although not conclusively, to be incorrect and must be fixed. Some of these defects can have been introduced on purpose by requirements engineers and that the final decision can be made only in the next software development activities.

\subsection{1.}

\section{Traceability between Petri-Net and Scenario}

Every transition in a Petri-Net denotes an event occurrence (episode sentence or exception solution) in its corresponding scenario. Every place denotes the location of a pre-condition, post-condition, cause or constraint. If event labels, condition labels and constraint labels are assigned to these Petri-Net transitions and places accordingly; then, defects in Petri-Net can be translated to defects in scenario:

- Non-interferential: Simultaneous enabled transitions represent simultaneous enabled episode sentences or exception solutions;

- Boundedness: An overflowed place represents a pre-condition, postcondition, cause or constraint;

- Liveness: A path to deadlock represents an ordered sequence of episode sentences or exception solutions from scenario initial state;

\section{6.}

\section{Recommending Fixes for Defects}

The final activity is that of giving advice to requirements engineers about the defect detected by our scenario analysis approach. Given the detailed information provided by our scenario analysis approach (e.g., it indicates the source of the defect), we have developed a rule-based heuristic as part of our scenario analysis approach in order to recommend fixes to requirements engineers, so that they can review scenario descriptions and deal with defects that hurt the properties related to unambiguity, completeness and consistency via refactoring of scenarios. A similar strategy based on recommendation tables was proposed by Rago et al. (2014).

Below, we list some general heuristics for generating useful recommendations. Table 21, Table 22, Table 23 and Table 24 show the recommendation to be provided by our analysis approach if some defect indicator 
is found within internal scenario elements or scenario's relationships. The last three columns of the table contain the property evaluation heuristic, defect indicators detected by the heuristic, and the recommendation given to the requirements engineers under the given defect indicator. It is up to the requirements engineers to decide whether a defect is correctly detected with our automated analysis approach and if they should follow the recommendation to fix that defect.

Table 21 - Recommendations for Analyzing Unambiguity Properties.

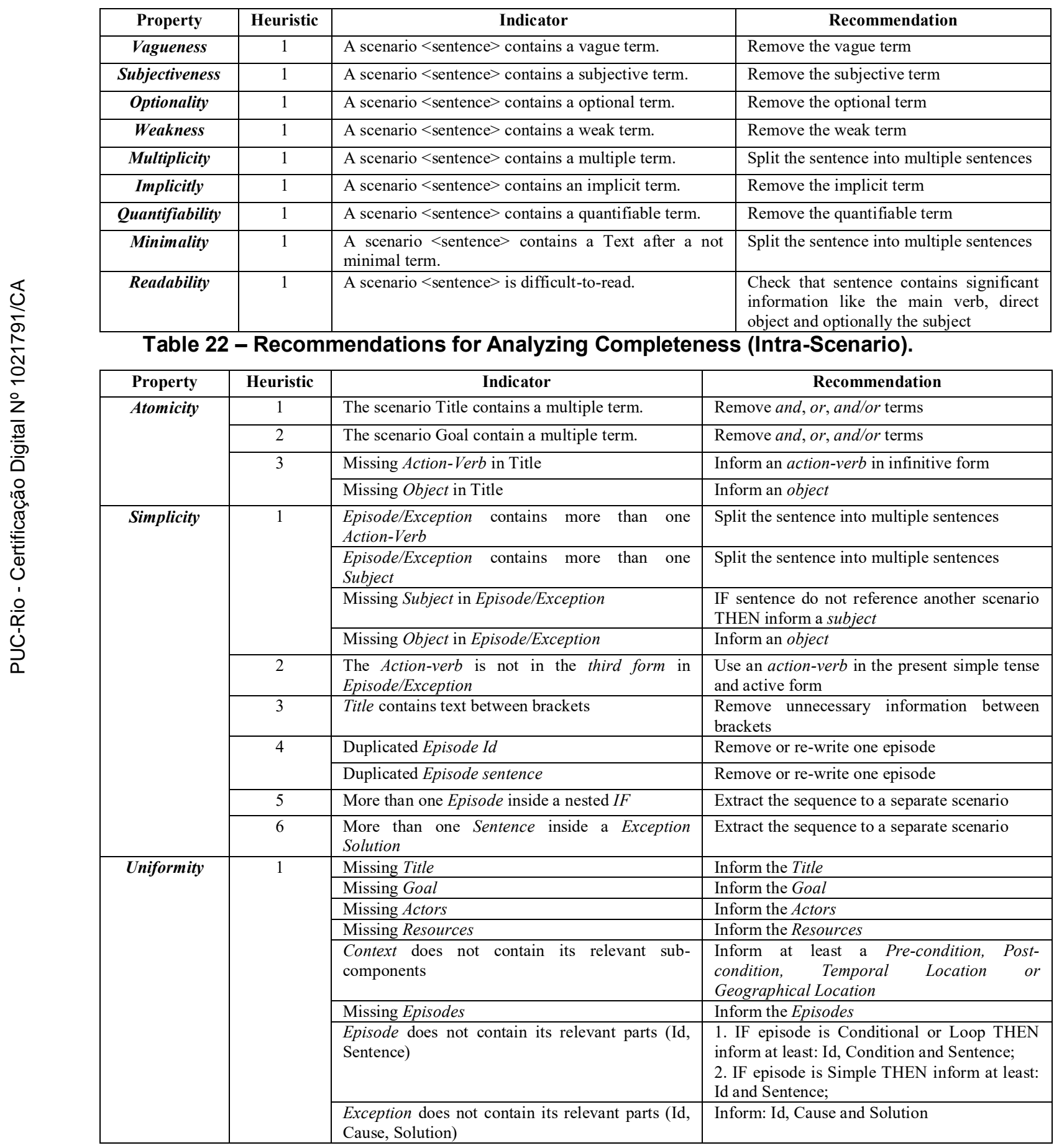


Table 23 - Recommendations for Analyzing Completeness (Intra-Scenario).

\begin{tabular}{|c|c|c|c|}
\hline Property & Heuristic & Indicator & Recommendation \\
\hline \multirow[t]{4}{*}{ Usefulness } & 1 & Actor does not participate in the situation; & Mention the actor in at least an episode \\
\hline & 2 & Missing Actor in Actors element; & Include the actor in the Actors \\
\hline & 4 & Missing Resource in Resources element; & Include the resource in the Resources \\
\hline & 5 & Branching Episode of an exception is missing; & $\begin{array}{l}\text { Update the exception Id to appoint the correct } \\
\text { episode }\end{array}$ \\
\hline \multirow[t]{5}{*}{$\begin{array}{l}\text { Conceptually } \\
\text { Soundness }\end{array}$} & 1 & $\begin{array}{l}\text { The corresponding verbs and objects appearing in } \\
\text { the two compared sentences are not the same }\end{array}$ & Re-write the Title to satisfy the Goal \\
\hline & 2 & & \\
\hline & 3 & & \\
\hline & 8 & Missing Action-Verb in exception solution; & Inform an action-verb \\
\hline & 9 & $\begin{array}{l}\text { Missing Linking-Verb or State-Verb in exception } \\
\text { causes; }\end{array}$ & Inform a linking-verb or state-verb \\
\hline
\end{tabular}

Table 24 - Recommendations for Analyzing Completeness (Inter-Scenario).

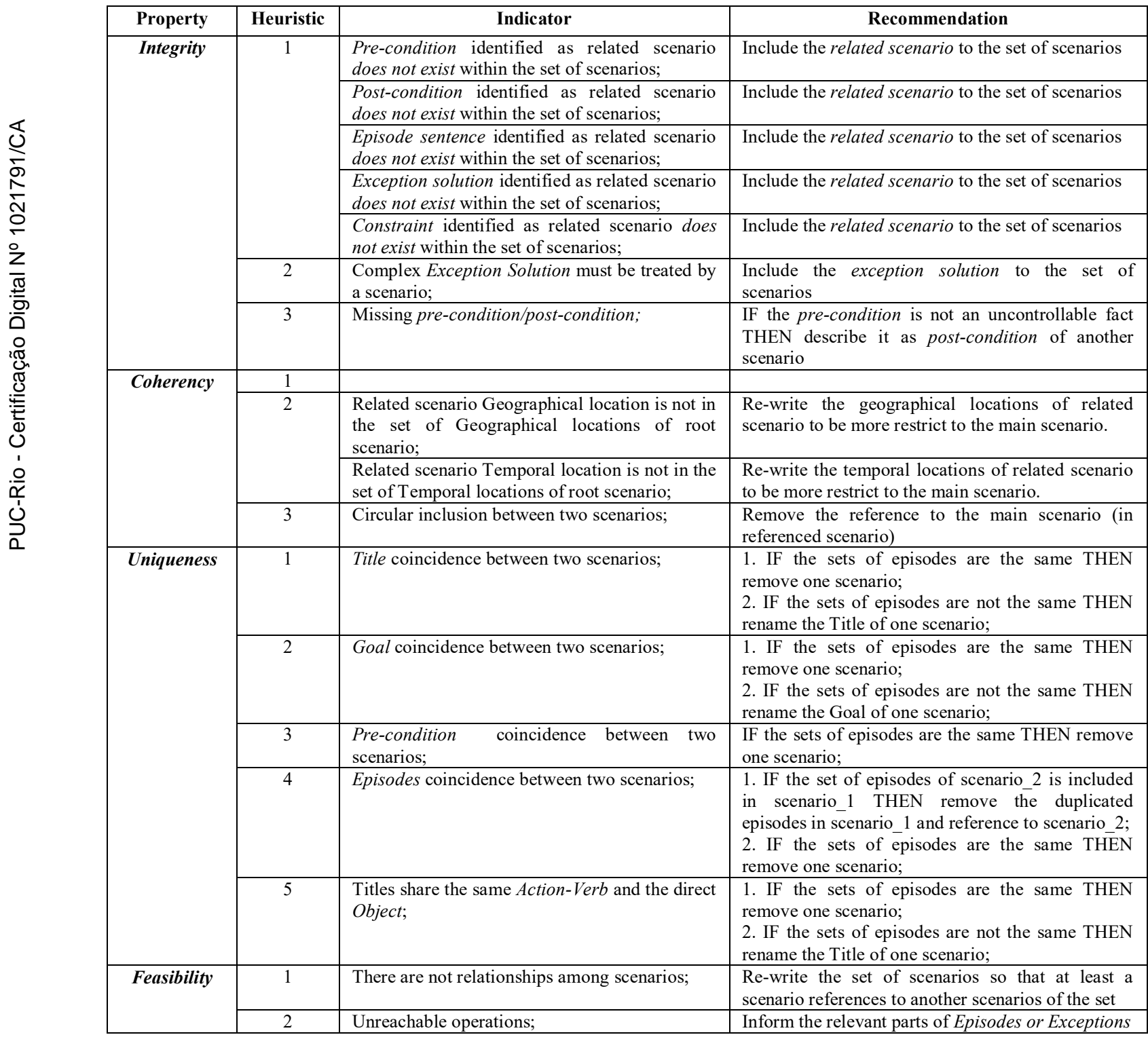


Table 25 - Recommendations for Analyzing Consistency Properties.

\begin{tabular}{|c|c|c|c|}
\hline Property & Heuristic & Indicator & Recommendation \\
\hline Non-interferential & 1 & Simultaneously enabled operations; & $\begin{array}{l}\text { 1. Check that all pre-conditions or constraints } \\
\text { associated to the episodelexception } \\
\text { corresponding to the transition are fulfilled; } \\
\text { 2. Notify to the next software development } \\
\text { activities; }\end{array}$ \\
\hline Boundedness & 1 & Overflowed resource; & $\begin{array}{l}\text { 1. Check that the overflowed resource is a } \\
\text { critical shared resource modified by several } \\
\text { scenarios; } \\
\text { 2. Notify to the next software development } \\
\text { activities; }\end{array}$ \\
\hline Reversibility & 1 & $\begin{array}{l}\text { There are no a path from an } \\
\text { operation to the initial state; }\end{array}$ & \\
\hline \multirow[t]{2}{*}{ Liveness } & \multirow[t]{2}{*}{1} & Path to deadlock & $\begin{array}{l}\text { 1. Check whether there are shared resources } \\
\text { modified by the scenarios and their } \\
\text { relationships; } \\
\text { 2. Notify to the next software development } \\
\text { activities; }\end{array}$ \\
\hline & & Never enabled transitions & $\begin{array}{l}\text { 1. Check that all pre-conditions, constraints, } \\
\text { conditions or causes of the episodelexception } \\
\text { corresponding to the transition are fulfilled; } \\
\text { 2. Notify to the next software development } \\
\text { activities; }\end{array}$ \\
\hline
\end{tabular}

Following are examples that explain the working of the recommendations in the scenarios of the "Online Broker System". Our analysis approach found a defect that hurts:

- Vagueness: The Episode 4 of the "Submit Order" scenario contains a vague term (indicator: "provided"). Therefore, the recommendation of our analysis approach is to "Remove the vague term";

- Simplicity: The Episode 1 of the "Local Supplier bid for order" scenario contains more than one Action-Verb (Indicator: "receives" and "examines"). Therefore, the recommendation of our analysis approach is to "Split the episode into multiple episodes";

Liveness: The Petri-Net corresponding to the "Submit Order" scenario contains a shortest path to deadlock (Indicator: <path from initial state to deadlock $>$ ). Therefore, the recommendation of our analysis approach is to "Check whether there are shared resources modified by the scenario and their relationships, and Notify to the next software development activities".

4.7.

Final Considerations

We presented a scenario language for describing scenarios using a Restricted-form of Natural Language (RNL). The proposed scenario language enables further transformation of scenario descriptions into executable design models like Petri-Nets, which can be used for more rigorous analysis tasks. 
In order to improve the results of analysis of scenario descriptions, we presented heuristics for finding non-explicit relationships among scenarios.

When we use any scenario for analysis, it is required to find and explore the related scenarios. However, it is difficult to ensure that all possible related scenarios are identified. To deal with this problem, we use the heuristics for finding relationships to explore the related scenarios.

We presented an approach for the analysis of scenarios through the use of NLP techniques and Petri-Nets. On the basis of this approach, it is possible to: (1) perform a static analysis to detect defects that hurt properties related to unambiguity and completeness; (2) perform a dynamic analysis to detect defects that hurt properties related to consistency, by executing equivalent Petri-Nets derived from scenarios and their relationships.

Our analysis approach provides modularity by first analyzing independent scenarios, then composing related scenarios to one component. Also, it supports traceability, indicating the defects in Petri-Nets and showing the source of the defects in scenarios (or their relationships).

It is important note that the transformation and integration of Petri-Nets methods do not introduce new defects such as described in Section 4.3.4.

\subsection{1.}

\section{Complexity Analysis}

The scenario language does not describe explicit iterations (it does not define "go to"); so that in most of cases, the Petri-Net $P N$ derived from a scenario will be an acyclic directed graph. In the Petri-Net derivation method (Method 1 Figure 26), the execution of a sequence of scenario episodes or exceptions is translated into a sequence of firing transitions. In the Petri-Net $P N$ all sequence of firing transitions are scanned from the initial places (initial marking $\boldsymbol{M}_{\boldsymbol{0}}$ ) to the final places (the set of reachable markings $\boldsymbol{M}$ ) using the DFS (Depth-first search) algorithm. The complexity of DFS is: $O(N+|E|)$ where ' $N$ ' is the nodes (|places $\mid+$ $\mid$ transitions $\mid)$ number and ' $|E|$ ' is the $\operatorname{arcs}$ number. The worst-case order is: $\mathrm{O}\left(N^{2}\right)$ where $|E|=N^{2}$. In scenarios (most case), each node has 2 outgoing edges (if- else for conditional/optional episodes), then $|E|=2 * N$, and the complexity of DFS be greatly reduced to: $\mathrm{O}(|E|)=\mathrm{O}(N)$. 
Only the integration of Petri-Nets method (Method 2 - Figure 27) induces an exponential complexity, because of the number of related Petri-Nets to be synthesized by fusing or substitution places or transitions. However, according to Somé (2010) this number is generally very limited in realistic examples. Thus, the complexity will be greatly reduced. It is $\mathrm{O}(N)$ when the synthesized Petri-Nets do not interact by non-sequential relationships.

According to Somé (2010), because scenarios describes requirements artifacts, the number of scenarios in projects is typically limited; therefore scaling to much larger projects should not be an issue because of the generally polynomial complexity of Petri-Net transformation and integration methods.

The case studies in Chapter 6 involve projects that specify between 5 and 36 scenarios with different degree of complexity, i.e., every scenario describes between 3 and 12 episodes, between 1 and 5 exceptions, and 1 concurrency construct (\#<episode series>\#). 


\section{5}

\section{C\&L (Cenários \& Léxicos)}

In this section we provide a description of $\boldsymbol{C} \boldsymbol{\&} \boldsymbol{L}-\boldsymbol{L u a}$ prototype tool, an integrated environment for supporting the analysis of natural language-based scenarios. It also provides the implementation strategies used to detect violation of properties related to Unambiguity, Completeness and Consistency.

\section{1.}

C\&L

C\&L prototype tool was developed at the PUC-Rio Requirements Engineering Group for editing and visualization of natural language-based scenarios and lexicon symbols. The Lua version of C\&L (C\&L) was developed by Almentero (2009).

Lexicon symbols are described using the Language Extended Lexicon (LEL). LEL is a language designed to help the elicitation and representation of the language used in the application. This model is based on the idea that each application has a specific language. Each symbol in the lexicon is identified by a name or names (synonyms) and two descriptions: Notion (denotation) explains the literal meaning - what the symbol is, Behavioral Response (connotation) describes the effects and consequences when the symbol is used or referenced in the application. Symbols are classified into four types: Subject, Object, Verb and State. Lexicon symbols are referenced within scenario descriptions. Table 26 shows the properties of a LEL symbol.

Table 26 - Symbol definition in lexicon language.

\begin{tabular}{|l|l|}
\hline Name & Symbol of LEL \\
\hline Type & Subject/Object/ Verb/State \\
\hline Synonymous & Term of LEL/Entry/Symbol \\
\hline Notion & $\begin{array}{l}\text { Word or relevant phrase of the Universe of Discourse. } \\
\text { It's described by Name, Type, } \text { Notion, Synonymous and Behavioral Response. }\end{array}$ \\
\hline $\begin{array}{l}\text { Behavioral } \\
\text { Response }\end{array}$ & $\begin{array}{l}\text { Its description contains the Type. } \\
\text { It has zero or more Synonymous. }\end{array}$ \\
\hline
\end{tabular}

In this thesis, lexicon symbols are not considered, but they can be used for further analysis of scenarios against application language represented in the lexicon. 
C\&L - Lua (Almentero, 2009) is a Web application developed in the Lua programming language (Ierusalimschy, 2013). The Kepler platform (Kepler, 2009) was used to develop C\&L because originally Lua was not designed for the development of Web applications. This platform provides a series of modules and tools which facilitates the writing of Lua code for the Web.

The C\&L - Lua architecture is based on the Model-View-Controller (MVC) framework. The architecture is vertically divided in layers and horizontally divided in modules. The modules are distributed in the view, controller and model layers, as can be seen in Figure 38. Four main modules were created from the scenarios that describe the situations of the application: User, Project, LEL and Scenario. These modules groups functionalities to manage users (User), projects (Project), lexicon symbols (LEL) and scenarios (Scenario).

The input of the C\&L - Lua is composed of projects containing scenarios or lexicons in plain text format. The output is a set of formatted scenarios and lexicons, where the relationships among scenarios or lexicons are represented by hyperlinks. It facilitates the navigation between scenarios and lexicons.

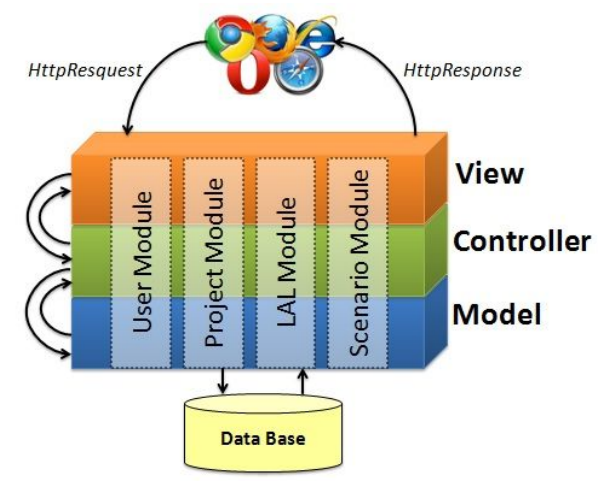

Figure 38 - C\&L - Lua Architecture (Sarmiento et al., 2014).

\section{2.}

\section{Extending C\&L - Lua}

C\&L - Lua was extended with the goal to provide an automatic support for the analysis of unambiguity, completeness and consistency qualities of scenarios described using a restricted-form of natural language (Section 4.1, Chapter 4). To reach this goal, we have implemented a set of modules and integrated a set of tools, each one dedicated to a specific analysis purpose of RNL scenarios. In particular, the involved tools are: (1) a NLP tool able to identify action-verbs, subjects and objects involved in scenario sentences; (2) a Petri-Net analysis tool 
able to simulate and detect overflows and deadlocks in translated scenarios; and (3) a Network Visualization tool having the aim of visualizing the equivalent Petri-Nets of scenarios. Figure 39 shows the high level architecture of the extended C\&L.

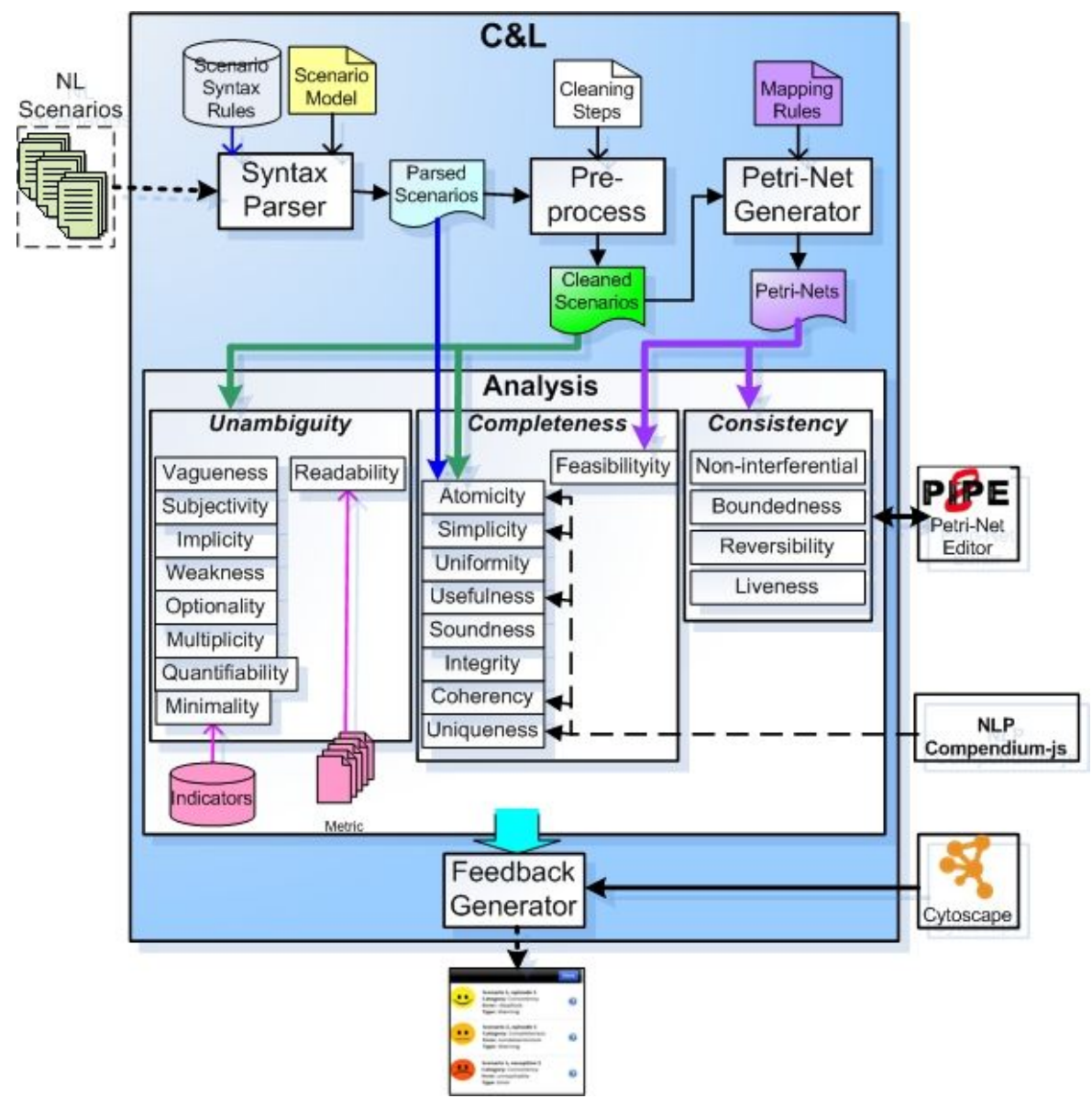

Figure 39 - High Level Architecture of Extended C\&L

\subsection{1.} Tools

During the implementation technologies strictly open source were mainly employed. Below the list of used tools together with short descriptions is presented:

- NLP Compendium-js: A Natural-Language-Processing library in Javascript, small-enough for the browser, and quick-enough to run on keypress (Compendium-js, 2015). It performs Part-of-Speech tagging (92\% on Penn Treebank, 2015), entity recognition, sentiment analysis and more. 
- Cytoscape: Cytoscape is an open source software platform in Javascript, for visualizing complex networks and integrating these with any type of attribute data (Cytoscape, 2015).

- PIPE2: An open source tool in Java, for creating and analyzing Petri-Nets (Place/Transition and Generalised Stochastic Petri-Nets). It detects defects that contribute to boundedness, safety and deadlock by analyzing the reachability graph (PIPE2, 2015).

\subsection{2. \\ Modules}

We extended the C\&L - Lua by adding the modules:

- Syntax Parser: Chunk textual scenario according to scenario model and syntax. This is necessary to enable the model's transformation.

- Pre-processing: Implement the steps for removing irrelevant information from scenario elements.

- Petri-Net Generator: Implement the Mapping Rules between scenarios and Petri-Nets.

- Analysis: Implement the methods to evaluate Structural (Static analysis) properties of scenarios and Behavioral properties (Dynamic analysis) of equivalent Petri-Nets:

○ Unambiguity: Evaluate scenario elements by searching defect indicators and applying metrics. For each property, common defect indicators are stored in dictionaries (Chapter 3, Table 7).

○ Completeness: Evaluate scenario elements by applying heuristics to search defect indicators (Chapter 3, Table 8, Table 9, Table 10 and Table 11). These heuristics are driven by syntax checks and by cross-referencing the related scenarios. Some properties (atomicity, simplicity, usefulness, conceptually soundness and uniqueness) are evaluated by Phrase-structure parsing and using a NLP tool.

- Consistency: Evaluate the behavior of a set of related scenarios by running executable equivalent Petri-Nets of scenarios and searching defect indicators (Chapter 3, Table 12). This evaluation is driven by the Reachability Analysis and using a Petri-Net tool. 
- Feedback Generator: Format the output of the analysis module by classifying defects found into Information, Warning or Error; and appointing the defect indicator and the fix recommendation for it. PetriNet defects are traced into Scenario defects. Additionally, this module uses a Visualization tool for enables the visualization of Petri-Nets in a modular way, i.e., Petri-Nets of related scenarios are grouped into separated nets and linked by common places.

\section{3.}

\section{Implementation Details}

In this sub section, we discuss the details of each module added to the C\&L - Lua. The implementation of each module was driven by scenarios and based on the process proposed by Almentero (2009). Each one of the situations performed by the modules was described using the scenario language presented in this work. The underlined terms (UPPERCASE) are references to other scenarios. Scenarios described in this chapter do not detail exceptional behavior.

The scenarios describing the situations of the remaining modules (User, Project, Lexicon and Scenario) are detailed in Almentero (2009).

\subsection{1.}

\section{Syntax Parser Module}

One of the main aspects of verifying whether a plain text represents a scenario description is splitting it into the main scenarios elements (Section 4.1, Chapter 4). This process consist of two steps: (1) Identify the main scenario elements by chunking on common text indicators such as TITLE, GOAL, CONTEXT, RESOURCE, ACTOR, EPISODES and EXCEPTION; and (2) Verify that every scenario element contains their relevant components, by chunking on common text indicators such as GEOGRAPHICAL LOCATION, TEMPORAL LOCATION, PRE-CONDITION, POST-CONDITION, CONSTRAINT, IF, THEN, WHILE, DO, AND, OR, MUST, NOT, “[”, "]" and "\#".

Each scenario element (Title, Goal, Context, Resource, Actor, Episodes, Exception) is decomposed in its main components and sub-components, i.e., each component is stored in a separate entity. String finding and regular expressions 
are used to perform this step. For example, an exception is decomposed in: $I d$, Cause (set of conditions) and Solution.

5.3.1.1.

Construct Scenarios

The main situations to verify the conformance of a plain text to the scenario model presented in Chapter 4 are described as scenarios. Figure 40 describes the steps to identify the scenario elements from a textual scenario.

\footnotetext{
TITLE: Identify Scenario Elements

GOAL: Produce a parsed scenario.

CONTEXT:

POST-CONDITION: Scenario elements are identified

ACTOR: C\&L

RESOURCES: scenario, scenario model

EPISODES

1. The C\&L identifies the Title element using the scenario model.

2. The C\&L identifies the Goal element using the scenario model.

3. The C\&L identifies the Context element using the scenario model.

4. The C\&L identifies the Actor element using the scenario model.

5. The C\&L identifies the Resource element using the scenario model.

6 . The C\&L identifies the Episodes element using the scenario model.

7. The C\&L identifies the Exception element using the scenario model.

8. The C\&L returns the semi-parsed scenario.
}

Figure 40 - Scenario to Identify the Scenario Elements

The steps to verify that every scenario element contains their main components is described by other scenarios, because each scenario element has particular components described using syntax rules. Figure 41,

Figure 42, Figure 43 and Figure 44 describe the situations to verify: Context, Resource, Episodes and Exception elements.

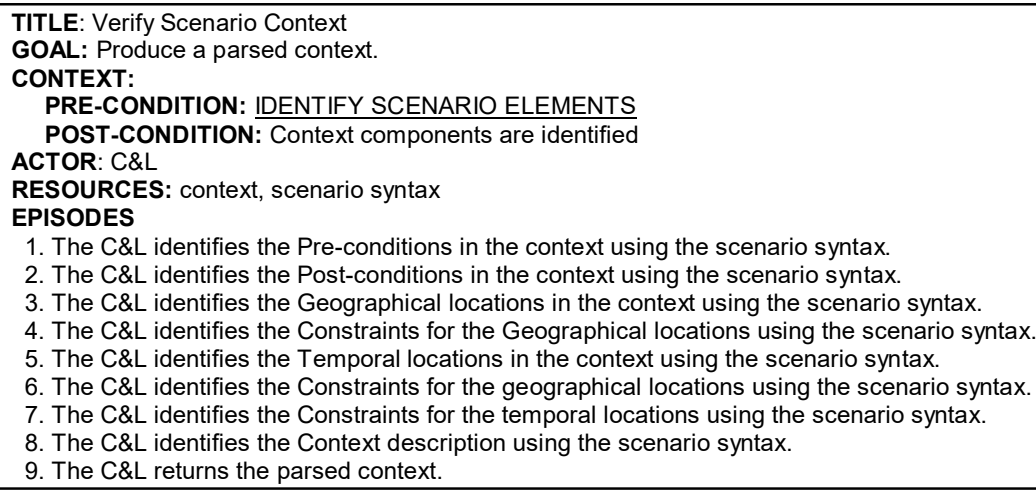

Figure 41 -Scenario to Verify the Main Components of Scenario Context

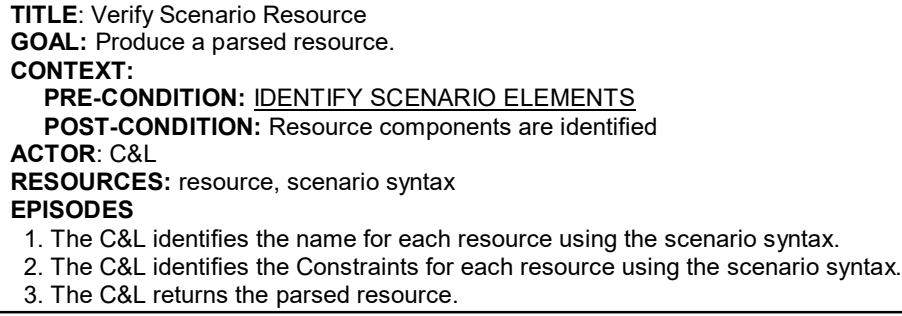

Figure 42 -Scenario to Verify the Main Components of Scenario Resource 
TITLE: Verify Scenario Episodes

GOAL: Produce a parsed list of episodes.

CONTEXT:

PRE-CONDITION: IDENTIFY SCENARIO ELEMENTS

POST-CONDITION: Episodes components are identified

ACTOR: C\&L

RESOURCES: episodes, scenario syntax

EPISODES

1. The C\&L identifies the Id for each episode in episodes using the scenario syntax

2. The C\&L identifies the Sentence for each episode in episodes using the scenario syntax.

3. The C\&L identifies the Conditions for each episode in episodes using the scenario syntax.

4. The C\&L identifies the Constraints for each episode in episodes using the scenario syntax.

5. The C\&L identifies the Pre-conditions for each episode in episodes using the scenario syntax.

6. The C\&L identifies the Post-conditions for each episode in episodes using the scenario syntax

7. The C\&L returns the parsed episodes.

Figure 43 - Scenario to Verify the Main Components of Scenario Episodes

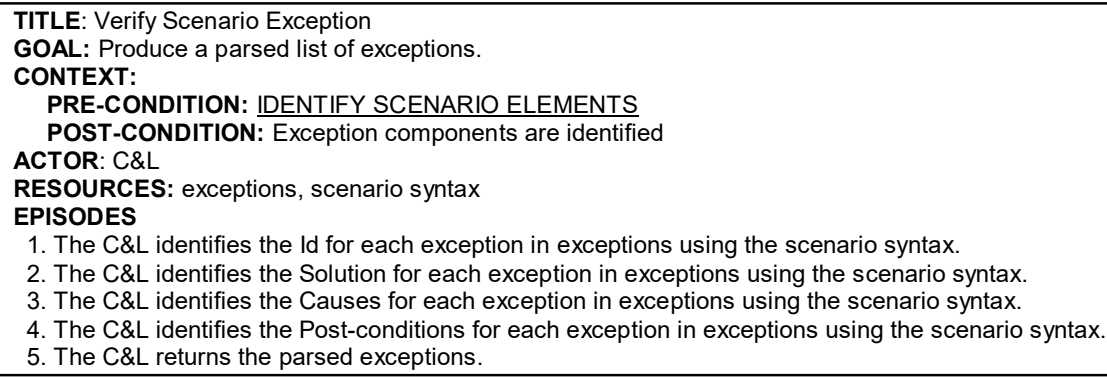

1. The C\&L identifies the Id for each exception in exceptions using the scenario syntax.

2. The C\&L identifies the Solution for each exception in exceptions using the scenario syntax.

3. The C\&L identifies the Causes for each exception in exceptions using the scenario syntax.

4. The C\&L identifies the Post-conditions for each exception in exceptions using the scenario syntax.

5 . The C\&L returns the parsed exceptions.

\section{Figure 44 - Scenario to Verify the Main Components of Scenario Exceptions}

\subsubsection{2. \\ Identify Root Scenario}

After scenarios for the Syntax Parser Module were constructed, It is necessary to identify the root scenario of this module. Thus, we first determine the relationship between the scenarios of the module, and from identified relationships we will establish an order between these scenarios. The root scenario will be the scenario that does not require any other scenario of the module (Almentero, 2009).

In this module we identify the relationships shown in

Figure 45. Based on these relationships we can determine that the scenario "Identify Scenario Elements" must precede all others. With this, we have identified the "Identify Scenario Elements" scenario as the root of this module.

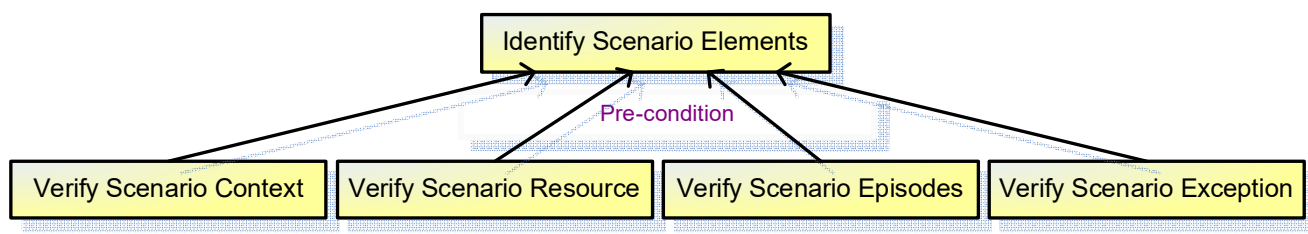

Figure 45 - Relationships among scenarios of Syntax Parser module 


\subsubsection{3. \\ Construct Integration Scenario}

In order to give an overview of the relationship among the several scenarios of the module, we construct an integration scenario. In an integration scenario, an episode corresponds to a sub-scenario.

The first step to construct the integration scenario of the module is the identification of the relationships between scenarios and the root scenario, and their order. We can see in

Figure 45 the relationships between the scenarios; the scenarios for verifying scenario elements can be executed in an indistinct or parallel order. Based on the order of the relationships, we create the integration scenario of the module. This scenario can be seen in Figure 46, and "Describe Scenario" scenario is a pre-condition for it (detailed in Almentero, 2009).

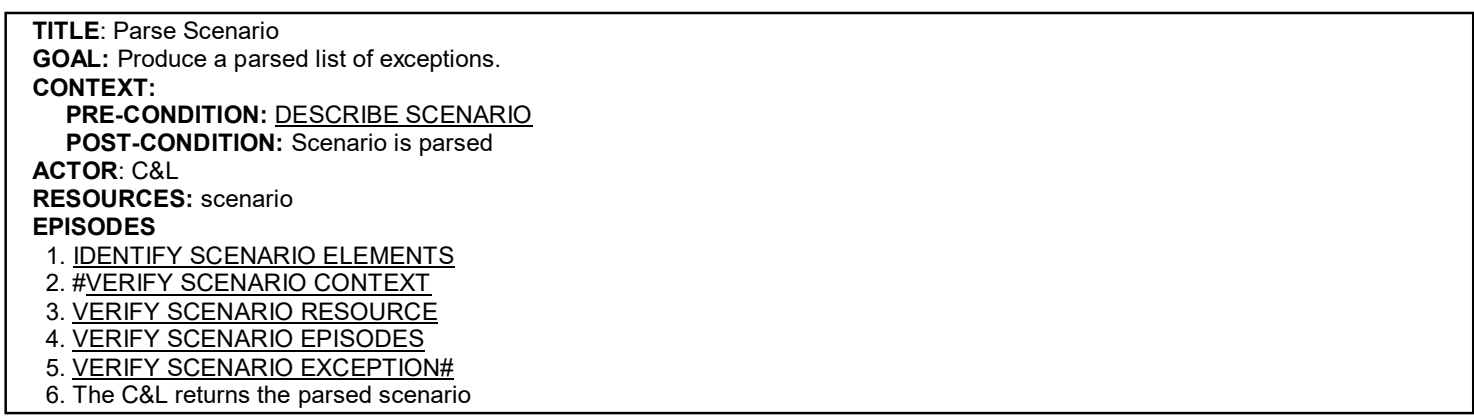

Figure 46 - Integration Scenario of Syntax Parser Module

\subsubsection{4}

\section{Operationalize Scenarios}

The scenarios of the Syntax Parser module are implemented by different methods and organized in model layer of the MVC framework.

We utilized string finding and regular expression matching to perform the sentences described in scenarios' episodes. For instance, two regular expressions are presented below:

- Regular expression used to separate each one of the actors or resources of a scenario:

$\circ \quad$ exp_reg_separ_items $="[\%, \% ;] "$.

○ For example: "actor1, actor2, actor3" $\rightarrow$ \{“actor1", "actor2", "actor3"\}. 
- Regular expression used to identify the ID of each one of the episodes or exceptions of a scenario:

$$
\begin{aligned}
& \text { ○ exp_reg_ids } \quad=\quad\left[\% \mathrm{o} * \% \mathrm{a}^{*}\right] \\
& * \% \mathrm{~d}+[\% . \%, \%: \% ; \% \mathrm{~s}] \% \mathrm{~d} *[\% . \%, \%: \% ; \% \mathrm{~s}] \% \mathrm{~d} *[\% . \%, \% ; \%:]^{*} \\
& \text { ○ For example: "1.1. IF Cause1 THEN Exception1" } \rightarrow \quad\{\mathrm{id}=" 1.1 . ", \\
& \text { Cause }=\{\text { "Cause1" }\} \text {, solution }=\text { "Exception1" }\} \text {. }
\end{aligned}
$$

\subsection{2.}

\section{Pre-processing Module}

One of the main aspects for translating scenarios into other models, or for analyzing scenarios using NLP tools, is removing irrelevant information from scenario elements (Chapter 4). This process consists of six steps: (1) Removal of Empty Line; (2) Removal of Capitalization; (3) Removal of Brackets; (4) Removal of URLs; (5) Removal of HTML Markup; and (6) Removal Punctuation.

\subsubsection{1.}

\section{Construct Scenarios}

Figure 47 presents the scenario that describes the steps to remove the scenario elements of irrelevant information. "Clean Scenario" is the root scenario of this module.

"Clean Scenario" is the root and integration scenario of this module.

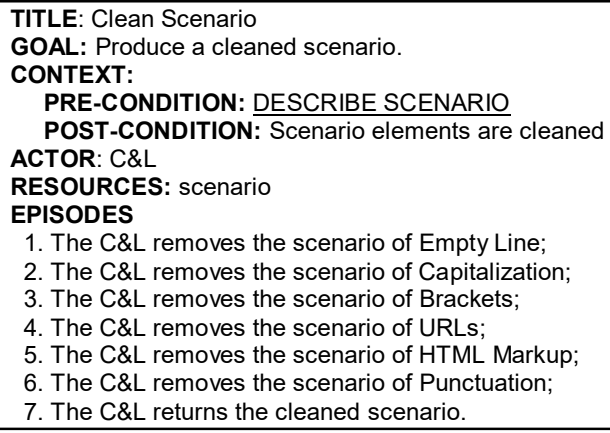

Figure 47 - Scenario to Clean Scenario of Irrelevant Information

\subsubsection{2.}

\section{Operationalize Scenarios}

We utilized regular expression matching to perform the actions described in "Clean Scenario" episodes. For instance:

- Regular expression used to remove brackets of a scenario sentence:

$\circ$ remove_parentheses_reg_ex $\left.={ }^{1} \%\left(\left[{ }^{\wedge}\right)\right]^{* \%} \%\right)^{\prime}$

○ remove_brackets_reg_ex $\left.=1 \%[\backslash[\wedge \%] \backslash]^{* \%} \%\right]^{\prime}$ 
O remove_braces_reg_ex $\left.={ }^{1} \%\{[\wedge 0 \%\}]^{* 0} \%\right\}^{\prime}$

○ For example: "The system displays the severity of alarm (high, medium, low)." $\rightarrow$ "The system displays the severity of alarm."

- Regular expression used to remove HTML Tags of a scenario sentence:

O remove_html_tags_reg_ex $==^{\prime}\left(\left\langle\left[^{\wedge}<>\right]^{*}>\right)^{\prime}\right.$

○ For example: "The system displays the name of the $\leq \mathrm{b}>$ alarm $\leq / \mathrm{b}>$ " $\rightarrow$ "The system displays the name of the alarm"

- Regular expression used to remove URL of a scenario sentence:

○ remove_url_reg_exp_http

'((https?)\%:[(\%/\%/)(\%\\%)]+[\%w\%d\%:\%\#\%@\%\%\%/\%;\%\$\%(\%) $\%$ \% $\%$ \% $\%$ \% $\left.\%+\%=\%=\% ! \% . \%: \%, \% \&]^{*}\right)^{\prime}$

○ remove_url_reg_exp_ftp $=$ '((ftp)\%:[(\%/\%/)(\%\\%)]+[\%w\%d\%:\%\#\%@\%\%\%/\%;\%\$\%(\%)\% $\%$ \% $\left.\% ? \% \backslash \%+\%-\%=\% ! \% . \%: \%, \% \&]^{*}\right)^{\prime}$

○ remove_url_reg_exp_file '((file)\%:[(\%/\%/)(\%〉\%〉)]+[\%w\%d\%:\%\#\%@\%\%\%/\%;\%\$\%(\%)\% \%_\%?\%\\%+\%-\%=\%!\%.\%:\%,\%\&]* $)^{\prime}$

○ remove_email_reg_exp $=$ '[A-Za-z0-9\%.\%\%\%+\%-]+@[A-Za-z0$9 \% . \% \% \%+\%-]+\% . \% \mathrm{w} \% \mathrm{w} \% \mathrm{w} ? \% \mathrm{w}$ '

○ For example: "The system sends an e-mail for the user user@mail.com" $\rightarrow$ "The system sends an e-mail for the user"

- Regular expression used to remove Punctuation of a scenario sentence:

○ remove_punctuation_exp_reg $=[[\% \mathrm{~s}+\%, \% . \%: \% ; \% ? \% ! \%=\%+\%$ $\% * \% / \% \# \% \$ \% \% \% \& \% \mid \%$ • $\left.\left(\%{ }^{\circ} \% \mathrm{~s}^{+}\right)\left(\%{ }^{\circ} \% \mathrm{~s}^{+}\right)\left(\% \% \mathrm{~s}^{+}\right)\left(\%{ }^{\circ} \% \mathrm{~s}^{+}\right)\left(\%{ }^{\prime} \% \mathrm{~s}^{+}\right)\right]^{\prime}$

○ For example: “6. ATM displays ¿New PIN Successful’ message.” $\rightarrow$ “6. ATM displays New PIN Successful message."

\subsection{3.}

\section{Petri-Net Generator Module}

One of the strategies to simulate a scenario description is mapping it into a Petri-Net (Chapter 4). This process consist of two steps: (1) Translate a scenario 
into a Petri-net by using mapping rules; and (2) Integrate the derived Petri-Net with Petri-Nets of related scenarios by using integration rules.

\subsubsection{1.}

\section{Construct Scenarios}

Each scenario element (Title, Goal, Context, Resource, Actor, Episodes, Exception) is mapped into Petri-Net nodes (places and transitions) and arcs. Mapping rules are used to perform this task. For example, an exception is mapped into a transition, input places and output places, which represent the exception solution, causes and post-conditions, accordingly.

Figure 48 presents the scenario that describes the steps to translate a scenario into a Petri-Net.

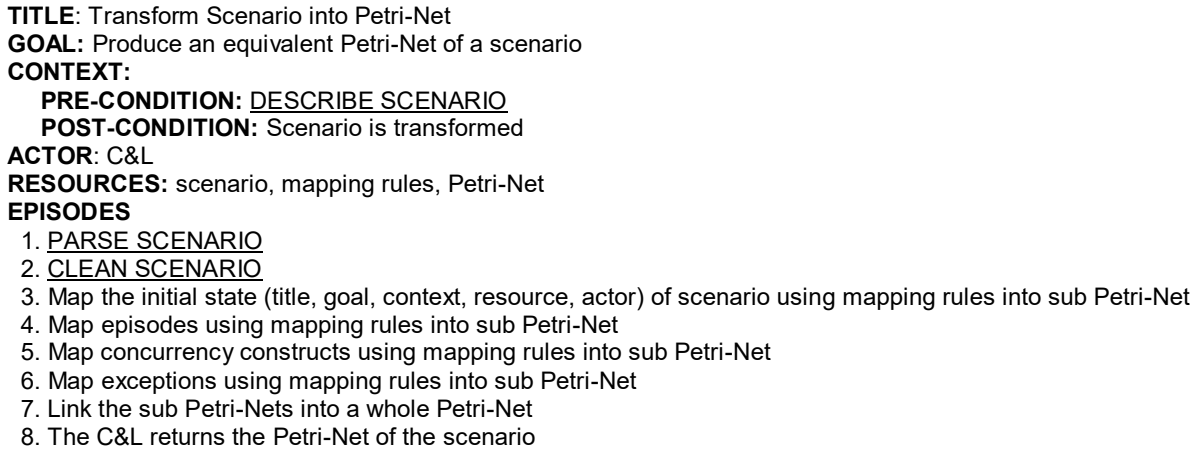

Figure 48- Scenario to transform a Scenario into a Petri-Net

A Petri-Net derived from a scenario and the Petri-Nets corresponding to related scenarios are integrated into a whole Integrated Petri-Net. Integration rules are used to perform this task.

Figure 49 presents the scenario that describes the steps to integrate a set of related Petri-Nets.

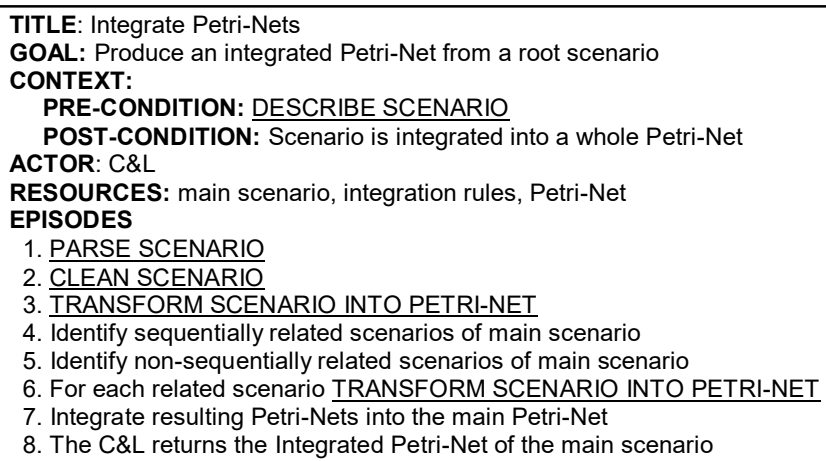




\subsubsection{2.}

\section{Identify Root Scenario}

In this module we identify the relationships among scenarios described in Figure 48 and Figure 49. Based on these relationships we can determine that both scenarios are root scenarios of this module, because none of them is pre-condition of the other.

\subsubsection{3.}

\section{Construct Integration Scenario}

Based on the relationships between scenarios and the root scenarios, we can see in Figure 49 that episode 3 references the "TRANSFORM SCENARIO INTO PETRI-NET" scenario, the relationships between these scenarios is by subscenario. Thus, "INTEGRATE PETRI-NETS" scenario is the integration scenario of the module.

\subsubsection{4.}

Operationalize Scenarios

The operationalization of the scenarios to transform a scenario into a PetriNet and integrate a Petri-Net with related Petri-Nets was presented in Chapter 4: Method 1 - Transform Scenario into Petri-Net and Method 2 - Integrate PetriNets.

We utilized mapping rules, fusion/substitution places and substitution of transition operations (Chapter 4: Table 15, Table 16, Table 17, Table 18, Table 19, and Figure 25) to translate a scenario and its relationships into a whole PetriNet.

\subsection{4.}

Analysis Module

The operationalization of the scenarios to analyze a scenario by detecting defects that hurt Unambiguity, Completeness and Consistency was presented in Chapter 4: Method 3 - Analyze Unambiguity, Method 4 - Analyze Completeness, and Method 5 - Analyze Consistency.

\subsubsection{1.}

\section{Construct Scenarios}

Figure 50, Figure 51 and Figure 52 shows the steps to verify the Unambiguity, Completeness and Consistency of a scenario, respectively. 
TITLE: Analyze Unambiguity

GOAL: Produce a list of unambiguity defects presents in a scenario.

CONTEXT:

PRE-CONDITION: DESCRIBE SCENARIO

POST-CONDITION: Scenario is analyzed

ACTOR: C\&L

RESOURCES: scenario, dictionaries

EPISODES

1. Check Readability index

2. Check Minimality using dictionaries

3. Check Vagueness using dictionaries

4. Check Subjectiveness using dictionaries

5. Check Optionality using dictionaries

6. Check Multiplicity using dictionaries

7. Check Quantifiability using dictionaries

8. Check Weakness using dictionaries

9. Check implicitly using dictionaries

10. The C\&L returns the list of defects of the scenario

Figure 50 - Scenario to Analyze Unambiguity

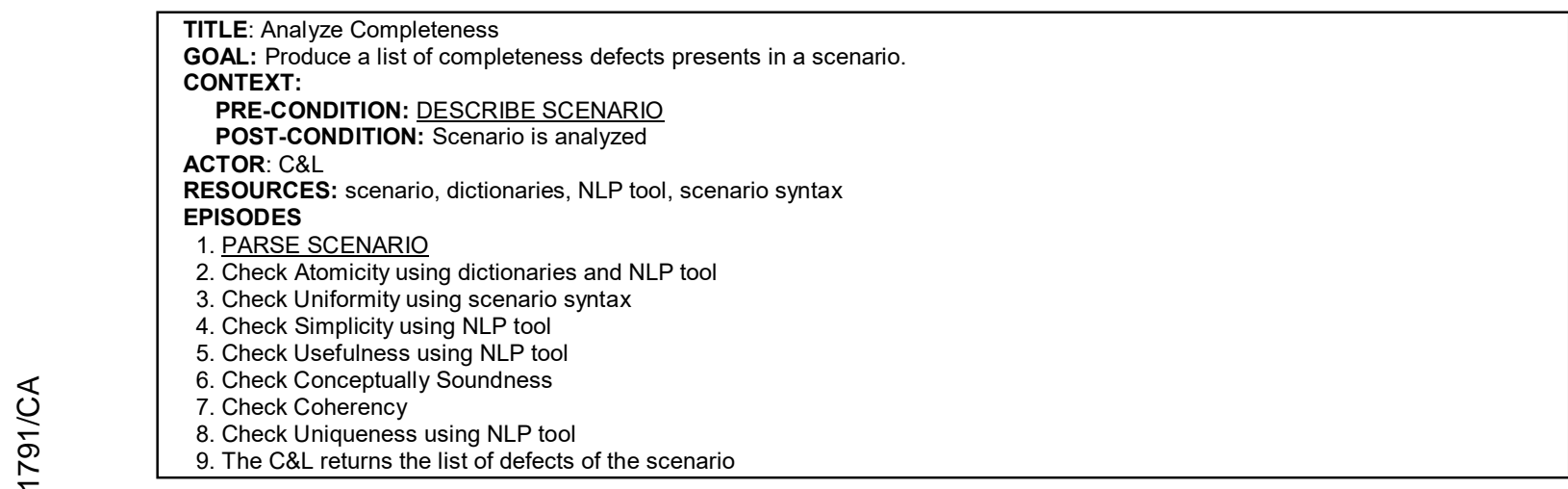

Figure 51 - Scenario to Analyze Completeness

TITLE: Analyze Consistency

GOAL: Produce a list of consistency defects presents in a scenario.

CONTEXT:

PRE-CONDITION: DESCRIBE SCENARIO

POST-CONDITION: Scenario is analyzed

ACTOR: C\&L

RESOURCES: scenario, Petri-Net tool

EPISODES

1. PARSE SCENARIO

2. INTEGRATE PETRI-NETS

3. Check Non-deterministic situations using NLP tool

4. Check Deadlock situations using NLP tool

5. Check irreversible situations using NLP tool

6. The C\&L returns the list of defects of the scenario

Figure 52 - Scenario to Analyze Consistency

\subsubsection{2.}

\section{Identify Root Scenario}

The scenarios of this module do not present relationships, then, we can determine that all scenarios are root scenarios of this module, because none of them is pre-condition of the other. 


\subsubsection{3.}

\section{Construct Integration Scenario}

Figure 53 presents the integration scenario that describes the steps to analyze a scenario. This scenario references in its episodes the integration scenarios of Syntax Parser, Pre-processing and Petri-Net Generator modules.

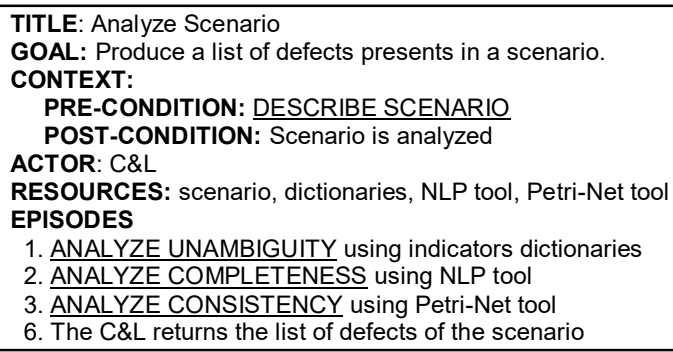

Figure 53 - Scenario to Analyze Scenario

\subsubsection{4.}

\section{Operationalize Scenarios}

We utilized String finding, Regular expression matching, Phrase-structure parsing, Levenshtein's distance (Levenshtein, 1966), Syntactic similarity heuristic, and Reachability analysis strategies to perform the tasks described in heuristics for finding defects.

\subsubsection{1. String Finding}

The string search operation is used to search a specific string within a scenario sentence. For example, this operation is used to search ambiguous indicators in scenario episodes.

- $\quad$ string.find(title, $<$ ambiguous indicator $>$ );

Heuristics for searching defect indicators of Unambiguity properties use String finding strategy and Coleman-Liau Readability metric.

Figure 54 depicts how Readability index (episode 1) and Weakness indicators (episode 8) are calculated and detected using the Lua language, respectively. 


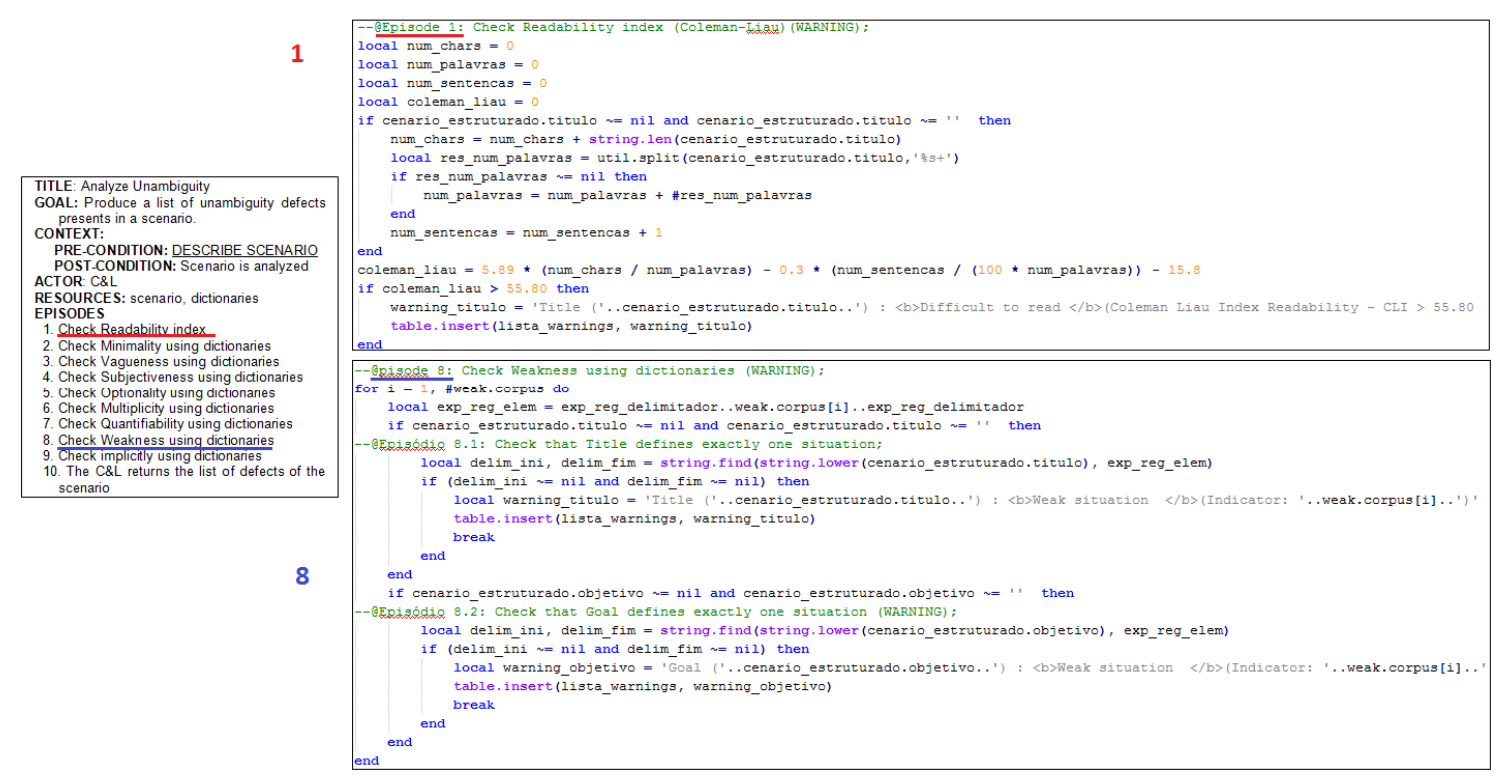

Figure 54 - String Finding Operationalization

\subsubsection{2. \\ Regular Expression}

This is a sequence of characters that forms a search pattern. The search pattern is used for text search in scenario sentences. For example, the following regular expression is used to search extra unnecessary information (text between parentheses) in scenario title:

- $\quad$ search_parentheses_reg_ex $=1 \%\left(\left[\left[^{\wedge}\right)\right]^{* \%} \%\right)^{\prime}$;

- string.find(title, search_parentheses_reg_ex);

\subsubsection{3.}

\section{Levenshtein's distance (Levenshtein, 1966)}

This strategy is a string metric for measuring the difference between two sequences. The distance between two words is the minimum number of singlecharacter edits (i.e. insertions, deletions or substitutions) required to change one word into the other. For example, this is used to measure the similarity between two scenarios by comparing their titles or objectives.

- Distance = levenshtein_distance $(<$ title $>,<$ goal $>)$;

\subsubsection{4. Phrase-structure Parsing}

This strategy is used to analyze the grammatical structure of sentences, and to identify which words are the Subject or Object of a main Verb. This strategy can indicate the forms of nouns and verbs found in a sentence. For example, this 
is used to check that scenario title contains an action-verb in infinitive form and an object.

Stanford parser (2015) tool is a most popular program to analyze the grammatical structure of sentences. This tool chunks a sentence into POS tags (Klein and Manning, 2003) and presents information about the relations between the Subject, Object and Verbs found in a sentence. In order to improve the accuracy, it is possible to train the parser by providing annotated data.

Figure 55 shows the tags used by NLP tools for tagging words in natural language-based sentences.

$\begin{array}{ll}\text { CC Coord Conjuncn } & \text { and, but, or } \\ \text { CD Cardinal number } & \text { one, two, 1,2 } \\ \text { DT Determiner } & \text { the, some } \\ \text { EX Existential there } & \text { there } \\ \text { FW Foreign Word } & \text { mon dieu } \\ \text { IN Preposition } & \text { of, in, by } \\ \text { JJ Adjective } & \text { big } \\ \text { JJR Adj., comparative } & \text { bigger } \\ \text { JJS Adj., superlative } & \text { biggest } \\ \text { LS List item marker } & 1 \text {, One } \\ \text { MD Modal } & \text { can, should } \\ \text { NN Noun, sing. or mass } & \text { dog } \\ \text { NNP Proper noun, sing. } & \text { Edinburgh } \\ \text { NNPS Proper noun, plural } & \text { Smiths } \\ \text { NNS Noun, plural } & \text { dogs } \\ \text { PDT Predeterminer } & \text { all, both } \\ \text { POS Possessive ending } & \text { 's }\end{array}$

$\begin{array}{ll}\begin{array}{ll}\text { PP Personal pronoun } \\ \text { PRP\$ Possessive pronoun }\end{array} & \begin{array}{l}\text { I, you, she } \\ \text { my, one's } \\ \text { quickly, not } \\ \text { RB Adverb }\end{array} \\ \text { RBR Adverb, comparative } & \text { faster } \\ \text { RBS Adverb, superlative } & \text { fastest } \\ \text { RP Particle } & +, \% \text {, \& } \\ \text { SYM Symbol } & \text { to } \\ \text { TO 'to' } & \text { oh, oops } \\ \text { UH Interjection } & \text { eat } \\ \text { VB verb, base form } & \text { ate } \\ \text { VBD verb, past tense } & \text { eating } \\ \text { VBG verb, gerund } & \text { eaten } \\ \text { VBN verb, past part } & \text { eat } \\ \text { VBP Verb, present } & \text { eats } \\ \text { VBZ Verb, present } & \text { which, that } \\ \text { WDT Wh-determiner } & \text { who, what } \\ \text { WP Wh pronoun } & \text { whose } \\ \text { WP\$ Possessive-Wh } & \text { how, where } \\ \text { WRB Wh-adverb } & \end{array}$

Figure 55 - NLP Tags (Compendium-js, 2015)

Parsing strategy returns a parse tree based on statistical analysis of POS tags; however, POS tagging strategies do not performs this task with high precision, such as demonstrated in Table 27. Table 27 shows the POS tagging results returned by Stanford (2015), NLTK (2015) and Compendium-js (2015) tools. The underlined tags are wrong answers pointed out by these tools.

Table 27 - tagging Examples using NLP Tools

\begin{tabular}{|c|c|c|c|}
\hline NLP tool & Sentence & POS Tags & Correct Answer \\
\hline Stanford & \multirow[t]{3}{*}{ Process bids } & Process/NN bids/NNS & \multirow[t]{3}{*}{ Process/VB bids/NNS } \\
\hline NLTK & & Process/NN bids/NNS & \\
\hline Compendium-js & & Process/ $\overline{\overline{N N}}$ bids/NNS & \\
\hline Stanford & \multirow[t]{3}{*}{$\begin{array}{l}\text { User downloads } \\
\text { the licence file }\end{array}$} & $\begin{array}{l}\text { User/NN downloads/NNS } \\
\text { the/DT licence/NN file/NN }\end{array}$ & \multirow{3}{*}{$\begin{array}{l}\text { User/NN } \\
\text { downloads/VBZ } \\
\text { the/DT } \\
\text { file/NN }\end{array}$} \\
\hline NLTK & & $\begin{array}{l}\text { User/NN downloads/NNS } \\
\text { the/DT licence/NN file/NN }\end{array}$ & \\
\hline Compendium-js & & $\begin{array}{l}\text { User/NN downloads/NNS } \\
\text { the/DT licence/NN file/NN }\end{array}$ & \\
\hline Stanford & \multirow{3}{*}{$\begin{array}{l}\text { Administrator } \\
\text { types in his user } \\
\text { name and } \\
\text { password }\end{array}$} & $\begin{array}{l}\text { Administrator/NNP types/NNS in/IN his/PRP\$ } \\
\text { user/NN name/NN and/CC password/NN }\end{array}$ & \multirow{3}{*}{ 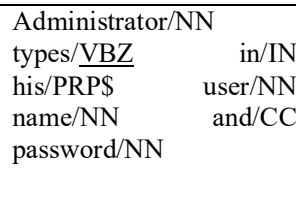 } \\
\hline NLTK & & $\begin{array}{l}\text { Administrator/NN types/NNS in/IN his/PRP\$ } \\
\text { user/NN name/NN and/CC password/NN }\end{array}$ & \\
\hline Compendium-js & & $\begin{array}{l}\text { Administrator/NNP types/VBZ in/IN his/PRP\$ } \\
\text { user/NN name/NN and/CC password/NN }\end{array}$ & \\
\hline
\end{tabular}


In Table 27, it is possible to notice that Stanford (2015) and NLTK (2015) tools did not identify the main verbs of three sentences. These verbs are tagged as "Nouns": "Process", "Downloads" and "Types". This fact is due to more than one Part-of-Speech can be associated with a word. For example in the sentence: Administrator types in his user name and password, the word types may be interpreted as a noun or verb.

Thus relying only on the parse tree may not provide good accuracy due to the imprecision of POS tagging phase. To improve the accuracy of parsing phase, we provide:

- Adjusting rules based on context-free grammars;

- Adjusting rules based on words that are both "Nouns" and "Verbs";

We noticed that sentences contain words that can be both a "Noun" and a "Verb". In fact, there are many words that can be used to name a person, place or thing and also describe an action. There are many examples of words that can be both nouns and verbs: "link", "step", "search", "contact", "validate", "approve", "download", "store", "delete", "use", "activate", "like", "form", "transfer", "view", "gra nt", "put", "display", "broadcast", "order", "process", "bid", "prompt", "update", "acce ss", "account", "release". More examples are listed in NounAndAdverb (2015).

Table 28 - Rules to Extract Action-Verbs and Nouns

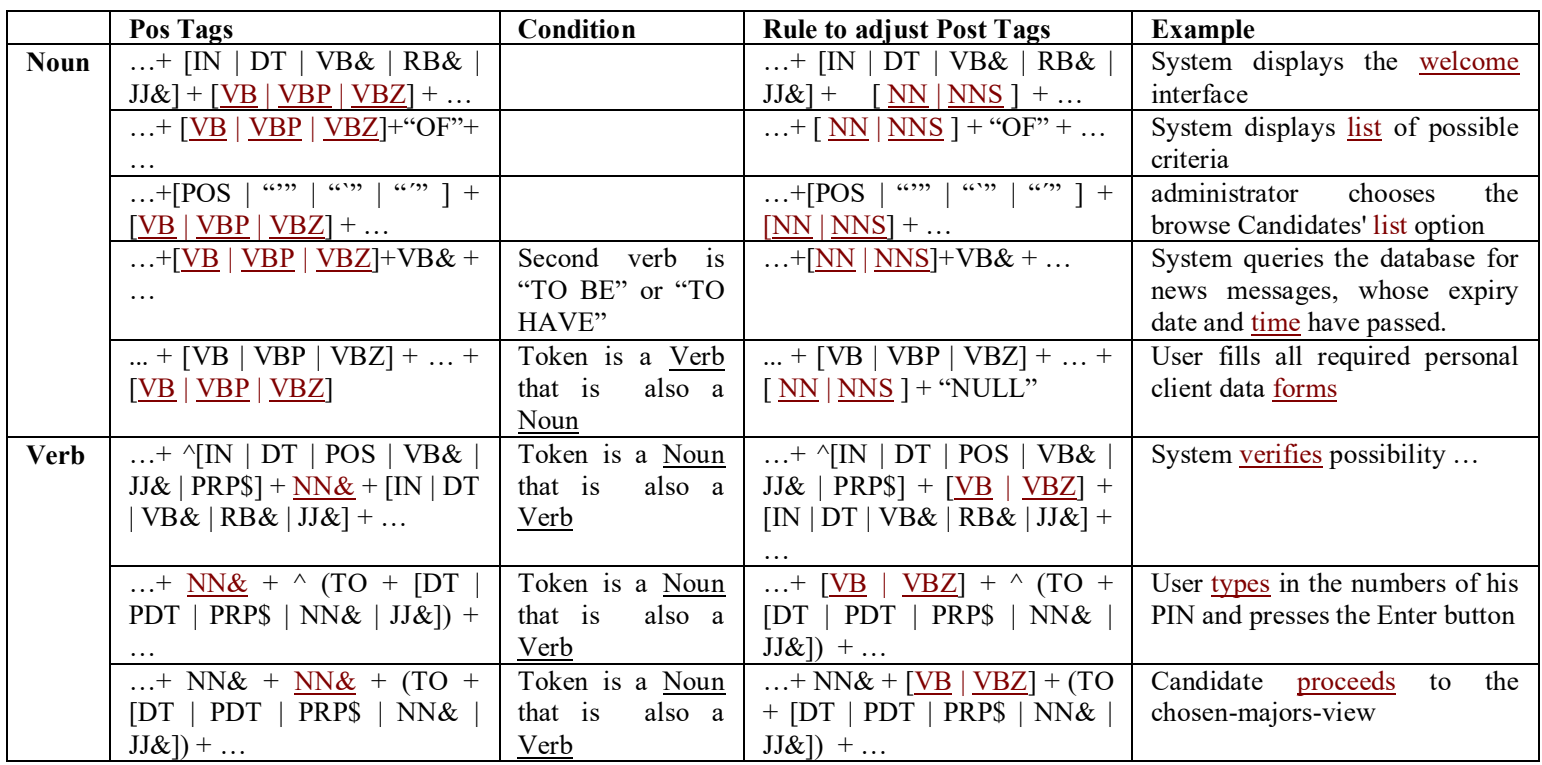

Table 28 shows the rules to adjust the accuracy of POS tagging phase adding a second phase. In Table 28, + means composition, () is used for grouping, | stands for "OR", $[\mathrm{x}]$ denotes the structure to select an option, and "^” denotes 
that is not contained within the brackets. "OF" is a terminal word and "..." means that a word is followed by other word.

Parsing strategy was used by Liu et al (2104) and Ciemniewska (2007) to extract action tuples information from use case steps. For improving the accuracy of parsing, they used annotated data for training the analysis on POS tags. However, this task requires an additional manual effort for training phase.

In this thesis, we improved the accuracy of parsing phase by creating simple rules to extract "Nouns" and "Verbs" based on dictionaries containing words that can be both a "Noun" and a "Verb". This strategy adjusts the accuracy of POS tagging phase and reduces the manual effort for training.

The tool Compendium-js (2015) provides the method "analyse (sentence)", which returns an object containing information like: POS tags and Tokens (Chunked text). Three methods were created for improving the POS tagging and Parsing tree:

- get_verbs $\left(<\right.$ sentence $>,<$ analise_sentence $>,<v e r b s \_$and_nouns $\left.>\right)$: Get action-verbs analysing POS tags, and using adjust rules detailed in Table 28 ;

- get_nouns $(<$ sentence $>,<$ analise_sentence $>,<$ verbs_and_nouns $>)$ : Get nouns analysing POS tags, and using adjust rules detailed in Table 28;

- get_sentence_components $(<$ sentence $>)$ : Get parse tree components (Action-verb, Subject and Object) using the action-verbs and nouns returned by previous methods;

Figure 56 depicts the implementation (JavaScript) of the method to get the main syntactic components of a textual sentence. 


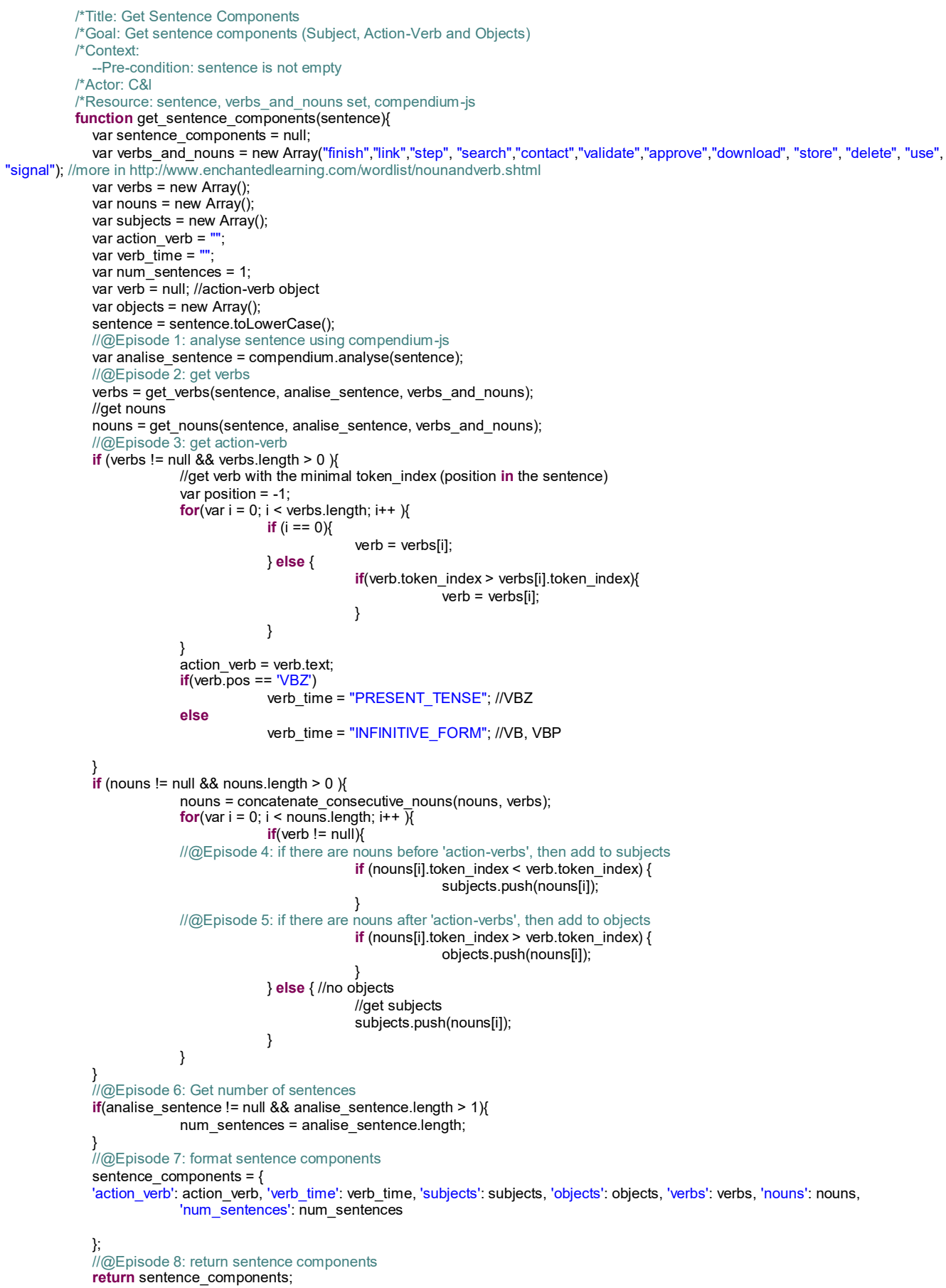

Figure 56 - Get sentence components method (Subject, Action-Verb and Objects).

\subsubsection{5. \\ Syntactic Similarity Heuristic}

This strategy is used to detect the syntactic similarity between two sentences. For example: for each verb and objects in the title of a scenario, it calculates the similarity with the verb and objects of another scenario title. We implemented the similarity heuristic by combining related works about modularization of requirements (Al-Otaiby et al., 2005) and similarity in user 
stories (Lucassen et al., 2015). We use a similarity measure that produces a value between zero and one, where zero means there is no relationship between the pairs of scenarios under question and one indicates a maximum relationship. The similarity between two scenarios $\boldsymbol{i}$ and $\boldsymbol{j}$ is calculated by the following steps:

- Calculate Object_Similarity $(\operatorname{title}(i)$, title $(j))=m / p$, where $p$ is total distinct objects in the two scenarios and $m$ is number of matching objects between the scenarios;

- Find Action_Verb(title(i)) and Action_Verb(title(j)) in the two scenarios;

- IF Object_Similarity(title(i), title(j)) >0 AND Action_Verb(title(i)) = Action_Verb(title(j)) THEN Scenario $\boldsymbol{i}$ and $\boldsymbol{j}$ are pontentially duplicated;

For example, "International Supplier bid for order" and "Local Supplier bid for order" are potentially duplicated, because they perform the same Action-Verb (bid) for the same Object (order).

Figure 57 depicts the implementation (JavaScript) of the method to measure the syntactic similarity between two textual sentences. 


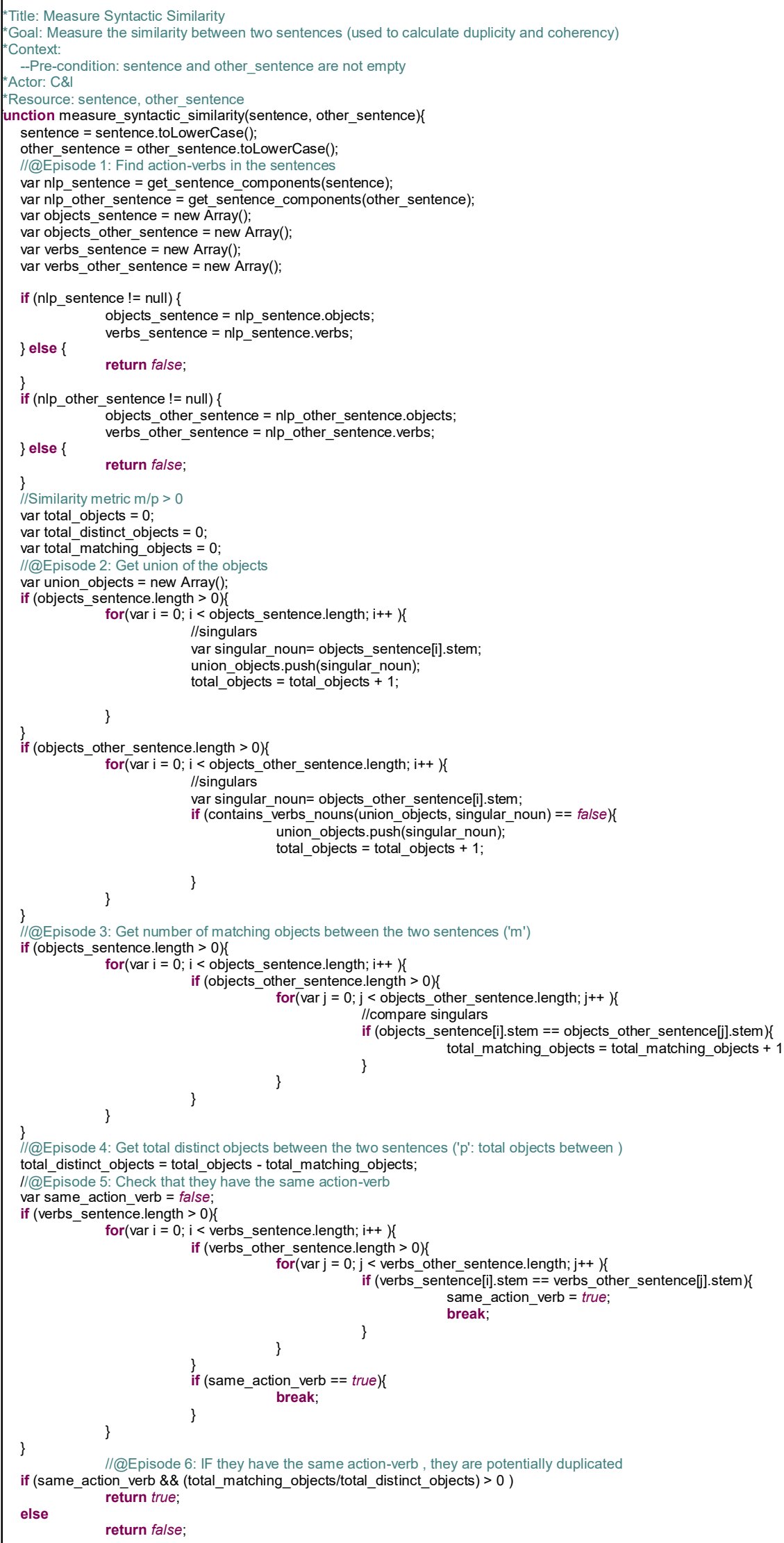

Figure 57 - Syntactic Similarity Implementation. 


\subsubsection{6.}

Reachability Analysis:

Heuristics for searching defect indicators that hurt Consistency properties use Reachability analysis strategy implemented in PIPE2 (2015) tool.

This strategy detects defects in Petri-Nets due to dynamic properties like Boundedness, Liveness and Deadlock-free (Reisig, 1985). In order to detect defects in Petri-Nets and indicate the source of these defects in scenarios and their relationships, we updated the PIPE2 (2015) tool by adding the "stateSpaceAnalysis" method to the module "StateSpace".

- pipe.modules.stateSpace.StateSpace: This module performs the reachabilty analysis of a Petri-Net in format PNML (Petri-Net Markup language);

- stateSpaceAnalysis(String pnmlFileName): Run state space using as parameter a filename (with pnml format). This method returns a feedback of the analysis, indicating the defects in Petri-Nets and their corresponding scenarios;

Other important method added to the class "MyTree" of calculations module is:

- Boolean[] neverEnabledTransitions(): This method returns a list containing never enabled transitions, when the Petri-Net is executed.

Figure 58 depicts the implementation ( $L u a$ ) of the method to run a java command line on Linux to run StateSpace analysis method of PIPE2 (2015). 


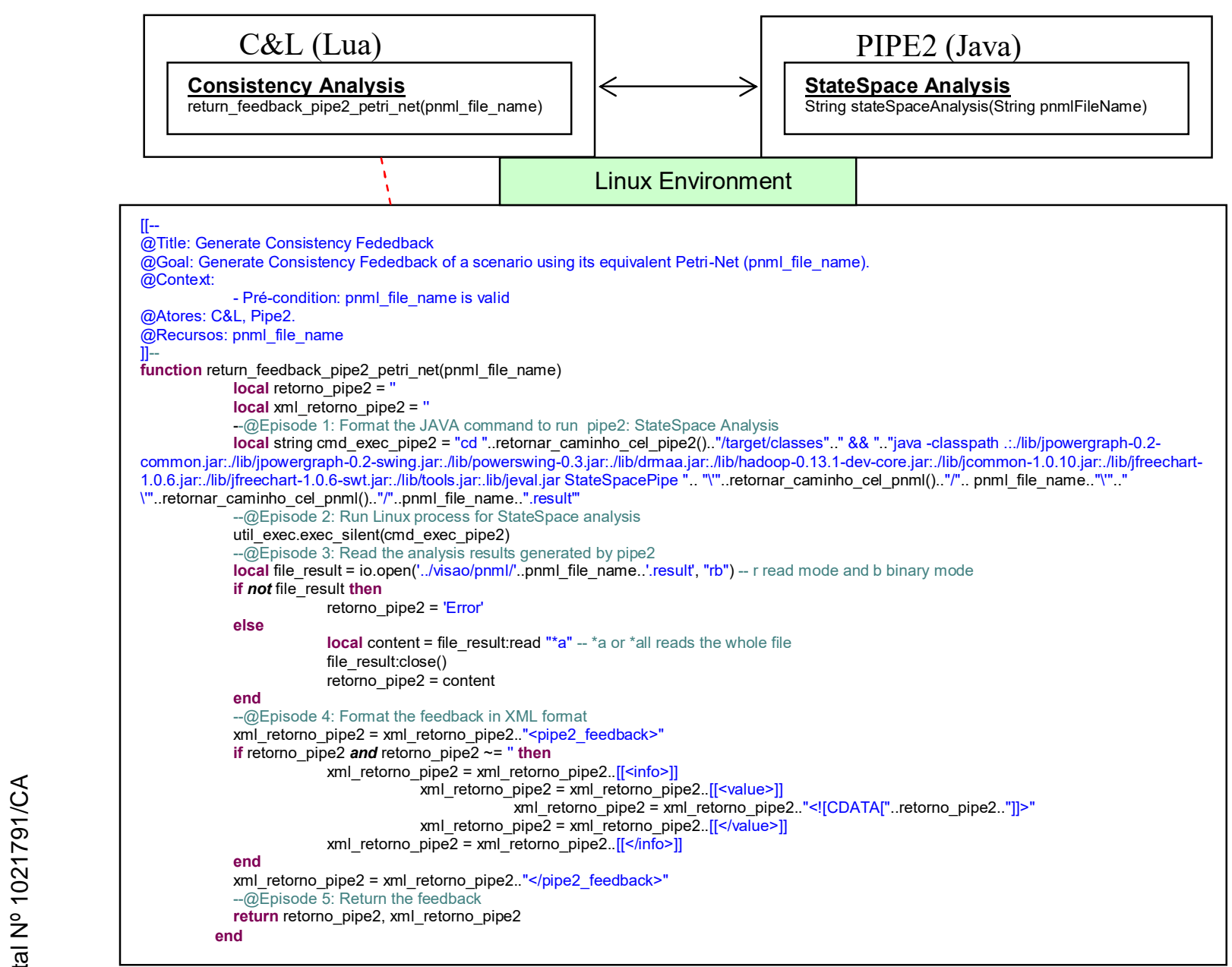

Figure 58 - Reachability Analysis on PIPE2.

Table 29 shows the implementation strategies for each one of the defect detection heuristics of Completeness properties.

\subsection{5.}

\section{Feedback Generator Module}

The output of the Analysis module must be formatted and returned to the requirements engineer. Thus, the defects are classified as Information, Warning or Error; and fix recommendations for each defect.

Figure 59 presents the scenario that describes the steps to format the analysis output of a scenario. 


\subsubsection{1.}

\section{Construct Scenarios}

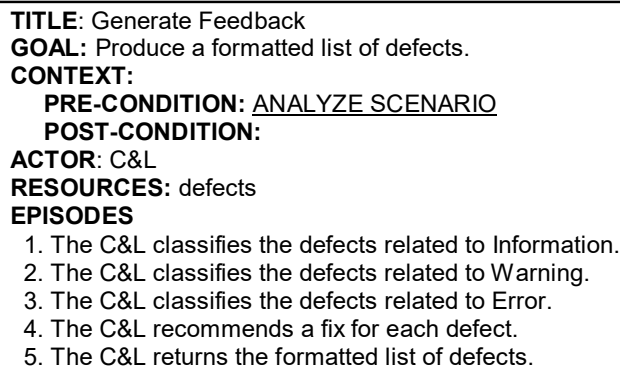

Figure 59 - Scenario to Generate Feedback

\subsubsection{2.}

\section{Operationalize Scenarios}

In Table 29, we detail how each one defect that hurts Completeness properties are classified, and how the heuristics to detect them are implemented and classified. The heuristics are classified according to the analysis strategy. The defects are classified as: Information, Warning or Error.

Defects that hurt Unambiguity properties are detected by String Finding strategy, and defects that hurt Consistency properties are detected by Reachability Analysis of corresponding Petri-Nets. 
Table 29 - Intra-scenario Properties Related to Completeness.

\begin{tabular}{|c|c|c|c|c|}
\hline Property & Heuristic & Analysis Strategy & Defect Category & Implementation \\
\hline \multirow[t]{3}{*}{ Atomicity } & 1 & Lexical & Warning & String finding \\
\hline & 2 & Lexical & Warning & String finding \\
\hline & 3 & Syntactic & Warning & Phrase-structure Parsing \\
\hline \multirow[t]{6}{*}{ Simplicity } & 1 & Syntactic & Warning & Phrase-structure Parsing \\
\hline & 2 & Syntactic & Warning & Phrase-structure Parsing \\
\hline & 3 & Lexical & Information & Regular expression matching \\
\hline & 4 & Lexical & Warning & String finding \\
\hline & 5 & Lexical & Warning & String finding \\
\hline & 6 & Lexical & Warning & String finding \\
\hline Uniformity & 1 & Lexical & Warning & $\begin{array}{l}\text { Regular expression matching, String } \\
\text { finding }\end{array}$ \\
\hline \multirow[t]{6}{*}{ Usefulness } & 1 & Lexical & Warning & String finding \\
\hline & 2 & Syntactic & Warning & Phrase-structure Parsing \\
\hline & 3 & Lexical & Warning & String finding \\
\hline & 4 & Syntactic & Warning & Phrase-structure Parsing \\
\hline & 5 & Lexical & Warning & String finding \\
\hline & 6 & Lexical & Warning & String finding \\
\hline \multirow{9}{*}{$\begin{array}{l}\text { Conceptually } \\
\text { Soundness }\end{array}$} & 1 & Syntactic & Warning & Syntactic Similarity Heuristic \\
\hline & 2 & Semantic & Warning & Difficult to be measured by an automatic \\
\hline & 3 & Semantic & Warning & tool; \\
\hline & 4 & Syntactic & Warning & Phrase-structure Parsing \\
\hline & 5 & Lexical & Information & String finding \\
\hline & 6 & Lexical & Information & String finding \\
\hline & 7 & Lexical & Information & String finding \\
\hline & 8 & Syntactic & Warning & Phrase-structure Parsing \\
\hline & 9 & Lexical & Information & String finding \\
\hline \multirow[t]{3}{*}{ Integrity } & 1 & Lexical & Error & String finding \\
\hline & 2 & Lexical & Information & String finding \\
\hline & 3 & Lexical & Information & String finding \\
\hline \multirow[t]{3}{*}{ Coherency } & 1 & Semantic & Warning & $\begin{array}{l}\text { Difficult to be measured by an automatic } \\
\text { tool; }\end{array}$ \\
\hline & 2 & Lexical & Warning & String finding \\
\hline & 3 & Lexical & Warning & String finding \\
\hline \multirow[t]{5}{*}{ Uniqueness } & 1 & Lexical & Warning & Levenshtein's distance \\
\hline & 2 & Lexical & Warning & Levenshtein's distance \\
\hline & 3 & Lexical & Warning & Levenshtein's distance \\
\hline & 4 & Lexical & Warning & Levenshtein's distance \\
\hline & 5 & Syntactic & Warning & Syntactic Similarity Heuristic \\
\hline \multirow[t]{2}{*}{ Feasibility } & 1 & Lexical & Error & Breadth-first search \\
\hline & 2 & Lexical & Error & Breadth-first search \\
\hline
\end{tabular}

5.4 .

Usage

The extended C\&L - Lua comes with simple web-based user interface. In the C\&L initial page (Figure 60) the user finds a small text explaining the software and links to external information about the C\&L.

To start using the C\&L the user must sign up. The user registration is done through a simple form were the user must provide, among other information, its 
name, e-mail, login and password. After registering in the application, the user can login informing the necessary data.

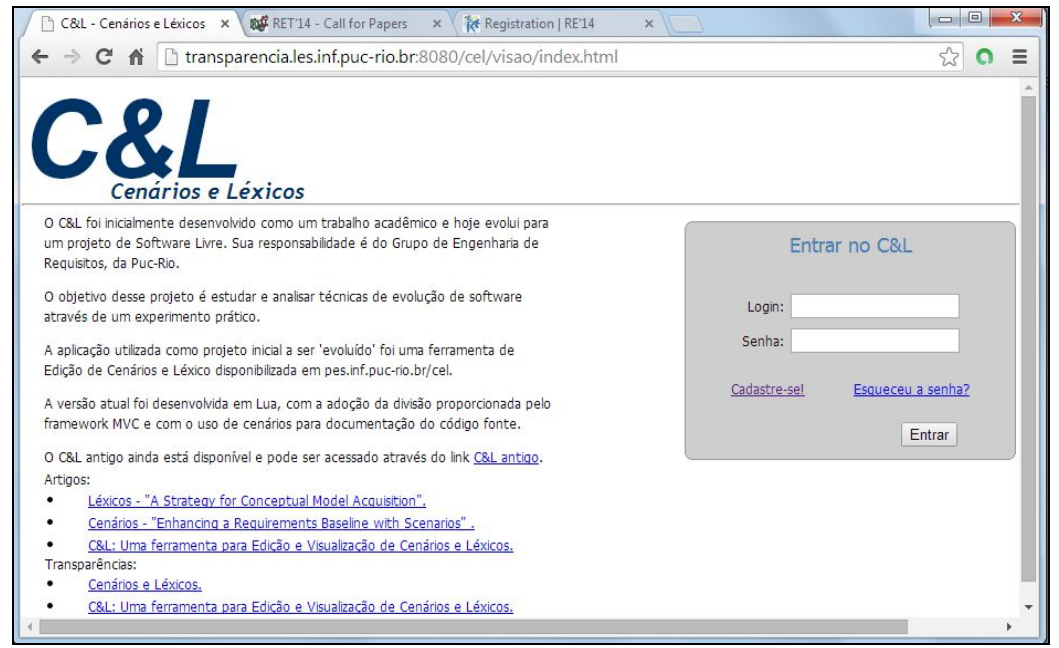

Figure 60 - Initial page of the C\&L.

\subsection{1.}

\section{C\&L Main Menu}

The operation of C\&L is described through an integration scenario (Figure 61), which is an artificial scenario created in order to provide an overview of the features of the software. The episodes of this scenario are references to scenarios that describe functionalities provided by the different modules that composes the system. The underlined terms are references to other scenarios (those that appear in uppercase) or to lexicon symbols (those that appear in lowercase). Thus, the term "SELECT PROJECT" in Figure 61 is a link to the scenario that describes how the user selects a project registered in the system. On the other hand, the term "user" that appears several times in the integration scenario, is a link to the description of the lexicon term whose name is "user". The concept of project is used within the system to represent different domains, where scenarios and lexicon symbols can be grouped.

After the user signing in, the system presents its main interface (Figure 61). In this interface there are many important elements to explore the system functionalities. These elements are: project, lexicon and scenarios menu, and work area. The first element is located at the top right of the interface. It is through this menu that the user selects the project he wants to work with at the moment. 


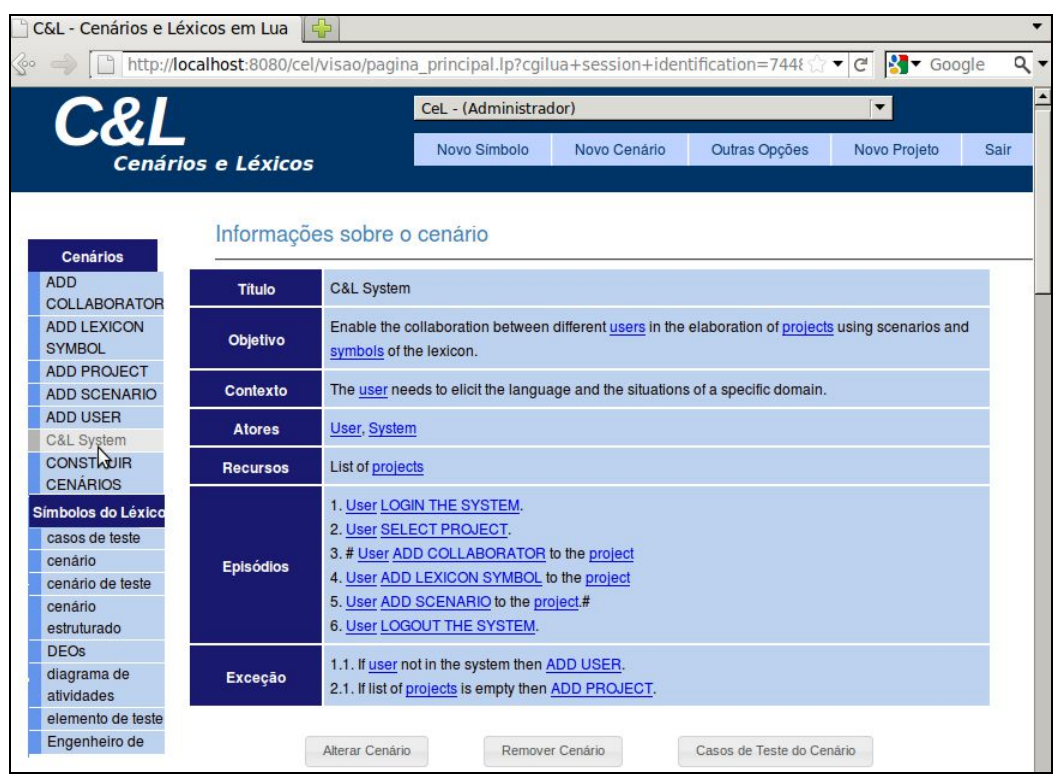

Figure 61 - Integration scenario to use the C\&L.

\subsection{2.}

\section{C\&L Scenario and Lexicon Functionalities}

Three new options appear at the main menu after selecting a project: new lexicon symbol, new scenario and other options. If the user selects the option "new scenario" he is redirected to a form (Figure 62) to include the information about the new scenario. The same happens when the "new lexicon symbol" option is selected, but in this case the form allows including information about a lexicon symbol. As the scenarios and lexicon symbols are included in the projects, their title and names are displayed in the lexicons and scenarios menu (left side of the main interface).

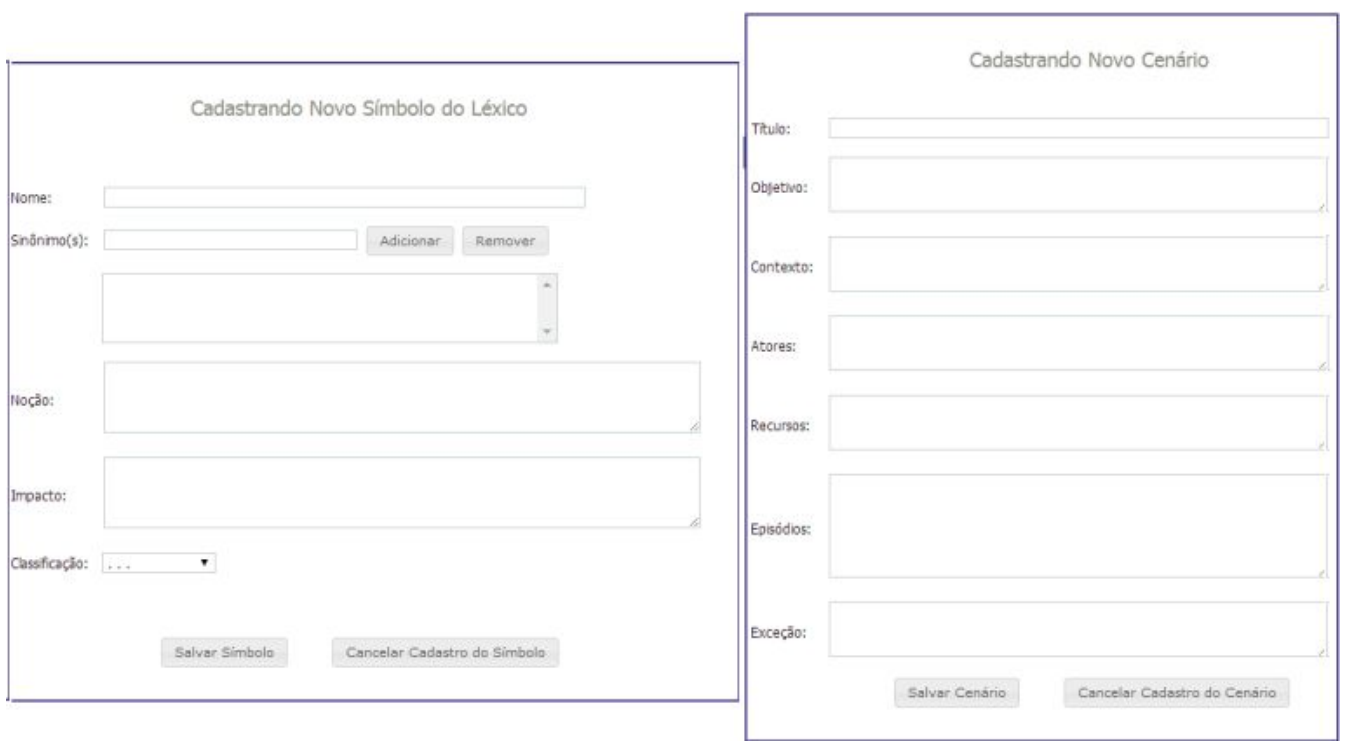

Figure 62 - Add lexicon symbol and add scenario forms. 
When the user selects a lexicon symbol or scenario from lexicons and scenarios menu, the C\&L application automatically assembles a network of relationships identifying what scenarios and lexicons symbols are referenced in the body of the selected element (Figure 61). The relationships identified are used to create two kinds of trace: backward and forward. These traces allow the navigation between the elements referenced by the one being visualized (forward) and the navigation between the elements that references the one being visualized (backward). The C\&L differentiates the links between scenarios (the scenarios are written in uppercase) and lexicon symbols (written in lowercase). The links between scenarios and scenarios, scenarios and lexicon symbols and lexicon symbols and lexicon symbols are created. Those from lexicons to scenarios are not created.

\subsection{3.}

\section{C\&L Analysis Functionality}

The scenario analysis functionality is activated in the project or scenario visualization interfaces (when the user selects a project or a scenario). This functionality generates a feedback containing a list of defects that hurt Unambiguity, Completeness and Consistency properties.

Figure 63 depicts the project visualization interface.

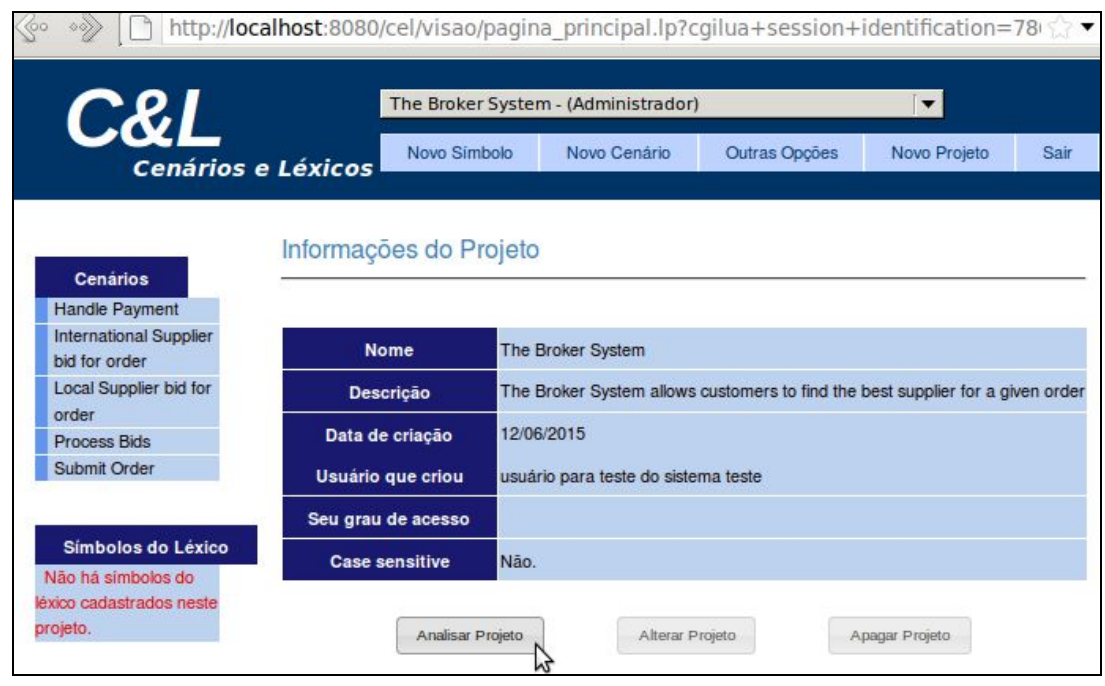

Figure 63 - Visualize Project Form. 


\begin{tabular}{|c|c|c|c|c|}
\hline Título & \multicolumn{4}{|l|}{ Submit Order } \\
\hline Objetivo & \multicolumn{4}{|c|}{ Allow customers to find the best supplier for a given order. } \\
\hline Contexto & \multicolumn{4}{|c|}{ PRE-CONDITION: The Broker System is online AND the Broker System welcome page is being displayed } \\
\hline Atores & \multicolumn{4}{|l|}{ Customer, Broker System } \\
\hline Recursos & \multicolumn{4}{|c|}{ Login page, Login information, Order } \\
\hline Episódios & \multicolumn{4}{|c|}{$\begin{array}{l}\text { 1. The Customer loads the login page } \\
\text { 2. The Broker System asks for the Customer's login information } \\
\text { 3. The Customer enters her login information } \\
\text { 4. The Broker System checks the provided login information } \\
\text { 5. The Broker System displays an order page } \\
\text { 6. The Customer creates a new Order } \\
\text { 7. DO the Customer adds an item to the Order WHILE the Customer has more items to add to the order } \\
\text { 8. The Customer submits the Order } \\
\text { 9. The Broker System broadcast the Order to the Suppliers } \\
\text { 10. \# LOCAL SUPPLIER BID FOR ORDER } \\
\text { 11. INTERNATIONAL SUPPLIER BID FOR ORDER \# } \\
\text { 12. PROCESS BIDS }\end{array}$} \\
\hline Exceção & \multicolumn{4}{|c|}{$\begin{array}{l}\text { 1.1 IF Customer is not registered THEN REGISTER CUSTOMER } \\
\text { 2.1 IF after } 60 \text { seconds THEN The Broker System displays a login timeout page } \\
4.1 \mathrm{IF} \text { the Customer login information is not accurate THEN The Broker System displays an alert message } \\
\text { 8.1 IF the order is empty THEN The Broker System displays an error message }\end{array}$} \\
\hline Alterar Cenário & Remover Cenário & Casos de Teste do Cenário & Analisar Cenánio & Analisar Cenário e seus Rejacionamentos \\
\hline
\end{tabular}

Figure 64 - Visualize Scenario Form.

When the analysis functionality is executed from the project interface, it analyzes each one of the scenarios of the project.

Figure 65 depicts the analysis feedback for the "Online Broker System" project described in Chapter 4.

\begin{tabular}{|c|c|}
\hline $\begin{array}{c}\text { Simplicity } \\
\text { (Intra-Cenário) }\end{array}$ & $\begin{array}{l}\text { - Episode (id = 9.): - Missing Action-Verb in Present Tense form (verbs: broadcast) } \\
\text { - Recommendation: Inform an action-verb in present tense } \\
\text { Núm. Infos: } 0\end{array}$ \\
\hline $\begin{array}{c}\text { Uniformity } \\
\text { (Intra-Cenário) }\end{array}$ & $\begin{array}{l}\text { Núm. Erros: } 0 \\
\text { Núm. Warnings: } 0 \\
\text { Núm. Infos: } 0\end{array}$ \\
\hline $\begin{array}{l}\text { Usefulness } \\
\text { (Intra-Cenário) }\end{array}$ & $\begin{array}{l}\text { Núm. Erros: } 0 \\
\text { Núm. Warnings: } 1 \\
\text { - Episodes : Too long scenario - Num. episodes }<10 \text { (Num. episodes: 12) } \\
\text { Recommendation: Re-write the scenario to keep between } 3 \text { and } 9 \text { episodes } \\
\text { - Episode (id = 10.): } \\
\text { - Actor/Resource (supplier) mentioned in episode is not included in the Actor/Resource element; } \\
\text { - Recommendation: Include the actor/resource in the Actors/Resources } \\
\text { Episode (id =11.): } \\
\text { - Actor/Resource (supplier) mentioned in episode is not included in the Actor/Resource element; } \\
\text { - Recommendation: Include the actoriresource in the Actors/Resources } \\
\text { Episode (id = 12.): } \\
\text { Núm. Infos: } 0\end{array}$ \\
\hline $\begin{array}{l}\text { Conceptually } \\
\text { Soundness } \\
\text { (Intra-Cenário) }\end{array}$ & $\begin{array}{l}\text { Núm. Erros: } 0 \\
\text { Núm. Warnings: } 0 \\
\text { - Title does not describe the Goal (potentially incoherent) } \\
\text { - Recommendation: Re-write the Title to satisfy the Goal } \\
\text { Núm. Infos: } 1\end{array}$ \\
\hline
\end{tabular}

Figure 65 - Project Analysis Feedback Interface (1). 
When the analysis functionality is executed from a specific scenario interface, it analyzes the scenario and the related scenarios (sequentially and nonsequentially related). Figure 66 depicts an excerpt of the analysis feedback for the "Submit Order" scenario of the "Online Broker System" project described in Chapter 4.

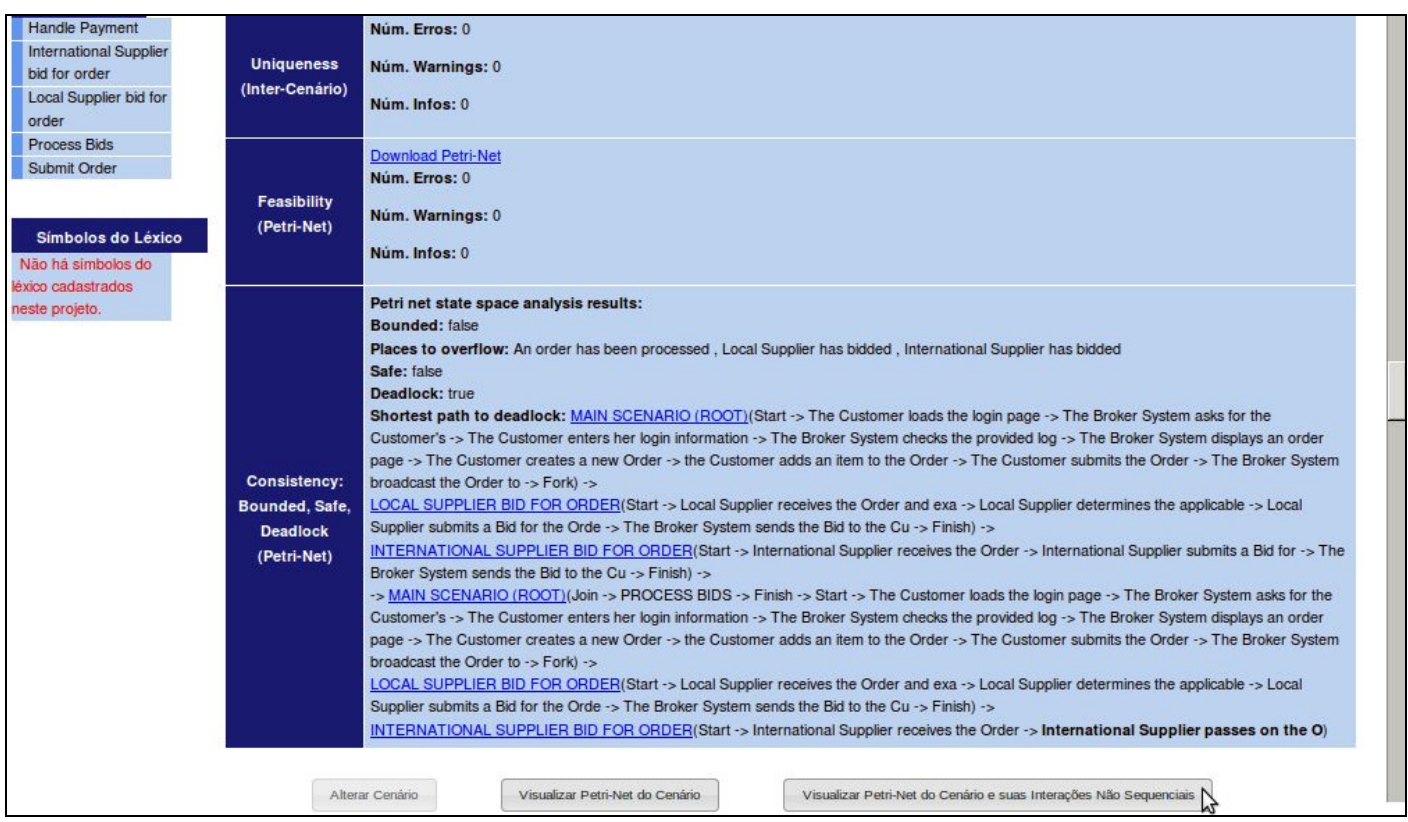

Figure 66 - Project Analysis Feedback Interface (2).

One important task of the feedback generator modules is to trace the defects reported from the Petri-Net tool (PIPE2, 2015), to the source of these in the scenarios. Figure 66 shows how a path to deadlock in the equivalent Petri-Net of the "Submit Order" scenario, is formatted using scenario elements, i.e. the path to deadlock is presented as a sequence of episodes and exceptions involving related scenarios.

\subsection{4.}

\section{C\&L Petri-Net Visualizer Functionality}

Other task of the feedback generator module is the visualization of the equivalent Petri-Net. From the analysis feedback form, we can activate the visualization interface. Figure 67 shows an excerpt of the integrated Petri-Net of "Submit Order" scenario. The places and transitions of the related Petri-Nets (related scenarios) are grouped in different modules. 


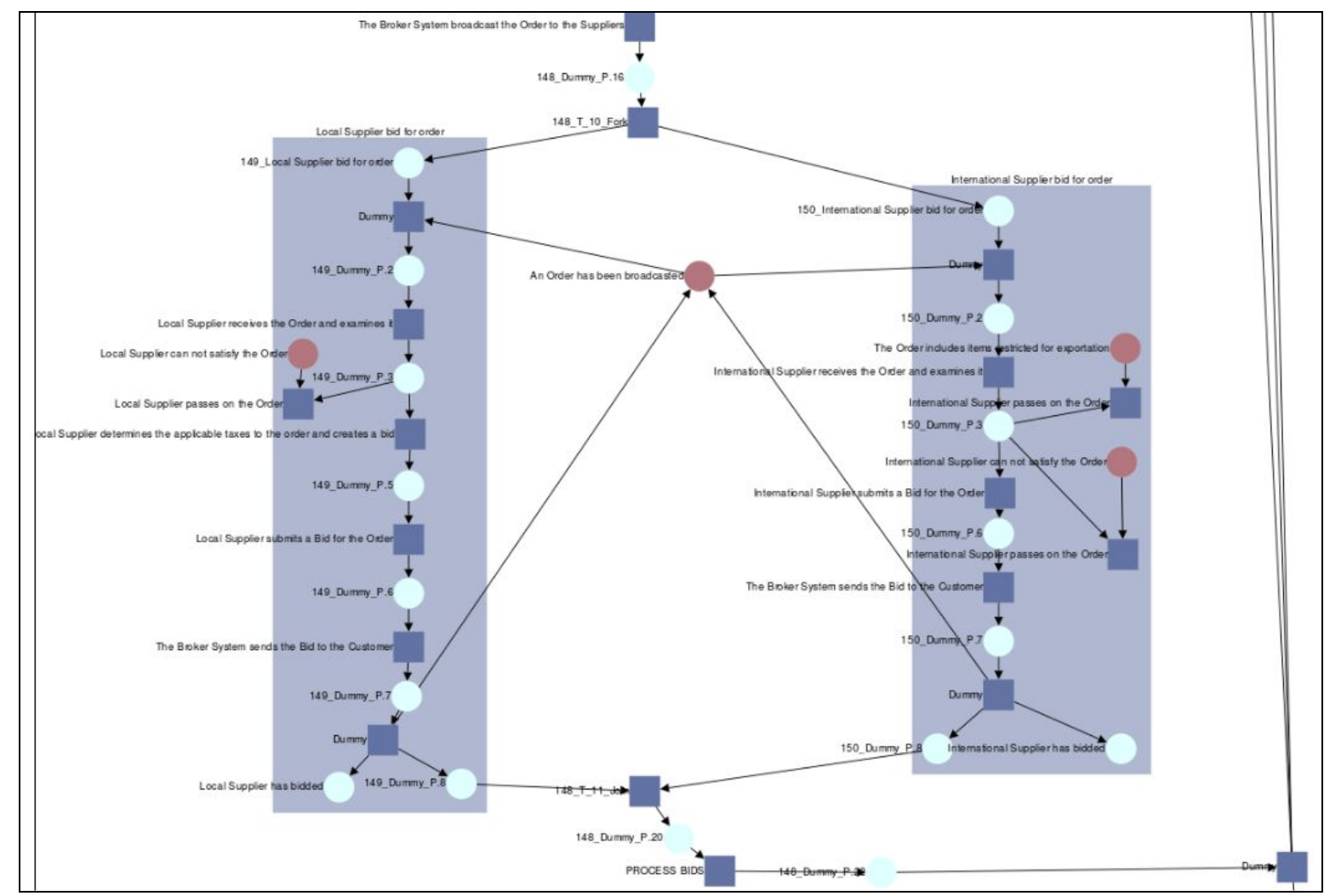

Figure 67 - Petri-Net Visualization Interface.

\section{5.}

\section{Final Considerations}

In this chapter we presented details of the implementation of the C\&L tool, a tool for supporting the tasks performed by our analysis approach. The objective of the extended C\&L is to assist the requirements engineers in their analysis tasks for improving scenario specifications.

Among the limitations of the C\&L are the heuristics (for finding defects) that depends on the NLP tool used (Compendium-js, 2015), which is a small version developed in JavaScript. In a further version, it is recommended the use an NLP tool with higher precision like Stanford (2015), openNLP (2015) or GATE (2015).

Other limitation is the method for measuring similarity between scenarios. It uses a syntactical analysis measure (Syntactic Similarity). In future version, we expect to enrich this method by considering synonymous information provided by the WordNet (2015) lexical database.

The implementation was tested on the unit level and on the integration level, using the examples of scenarios presented in case studies. These results will be shown in the next chapter. 


\section{6 \\ Case Studies}

In order to evaluate the effectiveness of the proposed approach, we apply our analysis approach, implemented in the $\boldsymbol{C} \boldsymbol{\&} \boldsymbol{L}-\boldsymbol{L} \boldsymbol{u} \boldsymbol{a}$ tool, to a set of scenarios from four different SRSs to show which defects are detected.

\section{1 .}

\section{Introduction}

This sub section describes the preparation needed to conduct the case studies.

The Goal of our case studies is to analyze SRSs described as scenarios with the purpose of detecting defects with close to $100 \%$ recall and higher precision. In line with Berry's notion of a dumb RE tool (Berry, 2012).

The case studies address the following research questions:

RQ1: Will the proposed automated analysis approach detect defects in SRSs in due time?

RQ2: Will the proposed automated analysis approach detect defects in the SRSs correctly and consistently?

\subsection{1. Hypothesis}

The general hypothesis of the case studies is that the proposed automated analysis approach should help to identify and show a great deal of defects from a set of scenario specifications, and furthermore take less time than if performed using experts (Requirements Engineers). We aim at evaluating our hypothesis with projects that cover a wide range of software domains, which apply use cases or scenarios for describing requirements, and which are publicly available for other researchers to compare their studies with ours. 


\subsection{2. \\ Variables}

In the case studies, we identified two response variables that will help to corroborate the hypothesis:

- The first variable measures the time needed to identify defects in a set of scenarios (Time Analysis).

- The second variable measures the amount of correct defects identified with our approach (Quality Analysis).

\subsection{3.}

\section{Evaluation Metrics}

Regarding the interpretation of the response variables, especially the second one, we chose to apply measures (precision and recall) from Information Retrieval (Olson, 2008). We used the definition of (Alchimowicz, 2011) for the description of the variables used in the precision and recall definition. Alchimowicz (2011) applied two other metrics: True positive rate and True negative rate.

Based on (Olson, 2008) we describe Precision and Recall:

- Precision measures the rate of correct defects identified by the approach (TP: true positives) in contrast to the amount of incorrect detections (FP: false positives).

- Recall measures the rate of correct defects identified by the approach (TP) in contrast to the amount of missed defects (FN: false negatives) from all the defects present in the set of scenarios.

Precision and Recall are computed as follows:

Precision $=T P / T P+F P$

Recall $=T P / T P+F N$

Based on (Alchimowicz, 2011), a defect within a scenario sentence can be classified as following:

- True Positive (TP): A defect is identified by the Requirements engineers and is detected by the approach (Defect occurs).

- True Negative (TN): A defect is not identified by the Requirements engineers and is not detected by the approach (Defect does not occur). 
- False Positive (FP): A defect is not identified by the Requirements engineers and is detected by the approach (Defect does not occur).

- False Negative (FN): A defect is identified by the Requirements engineers and is not detected by the approach (Defect occurs).

\subsection{4.}

\section{Case Study Selection}

As selection criteria for the four different SRSs, we took into consideration the following parameters:

- Access to a project in early stages of requirements specifications: We are interested in projects which apply use cases or scenarios for describing requirements, and that had already a preliminary analysis result, because we need an initial referential solution (baseline) for comparison.

- Ensuring reasonable diversity of domains: We are interested in projects that cover a wide range of software domains, i.e., type of project.

- Ensuring the availability of the project SRS in the community: We are interested in projects which are publicly available for other researchers to compare their studies.

- Ensuring reasonable scale: The number of scenarios must be reasonable.

Several real projects from the literature and their corresponding requirements specifications were analyzed for choosing the ones to be used in our case study that were representative enough for generalizing the findings of this evaluation. It is important to stress that each selected project did a case study to evaluate their analysis approach towards improving the quality of the project SRS.

As a result, we selected 4 projects, namely: Online Broker System (Somé, 2010), ATM System (Cox et al., 2004), DLibra (Ciemniewska and Jurkiewicz, 2007), and Mobile News (Ciemniewska and Jurkiewicz, 2007). These project's SRSs are described as use cases; so that, in order to evaluate them by our analysis approach, we need to translate them to scenario representations (using the 
scenario language proposed in this thesis). This task is carried out before constructing the referential solution (baseline) for each project.

The translation of use cases into scenarios does not introduce new defects, because use case components (pre-condition, post-condition, steps and alternatives) have corresponding components in the scenario language (precondition, post-condition, episodes and exceptions), and a sentence is written in a similar basic grammar (a basic sentence is composed of a 3-tuple subject-verbobject). These project's scenarios can be found in the Appendix 1.

The Online Broker System consists of use cases, whose goal is to allow customers to find the best supplier (Local and International) for a given order. These use cases were developed by Somé (2010) in order to evaluate an approach to formalize textual use cases using Petri-Net formalism, and detect inconsistencies.

The ATM System consists of a set of simplified use cases that describe the functionalities (for a supplier) to produce a new cash point for a major bank. There are five use cases described in total: access ATM, withdrawing cash, a customer can check their balance, make a deposit and change their PIN number. These use cases are available in Cox et al. (2004), and the authors introduced defects in order to evaluate a manual inspection technique based on checklists.

The DLibra and Mobile News consist of two sets of use cases created as a part of Software Development Studio (SDS) projects. SDS is one of the main components of the Master of Software Engineering program at Poznan University of Technology, Poland. The projects were developed for real customers from the university unit, industry or other organization. Their sources are available in the thesis of Ciemniewska and Jurkiewicz (2007). The case studies of these two projects comprise different software domains: (1) a Web-based Customer Relationship Management (CRM) system for managing the clients of a software for the creation of digital libraries (DLibra CRM), and (2) a news feed system for delivering the latest bulletins to mobile devices. The authors developed use case specifications containing typical defects from industrial projects. Then, they use these use cases to detect common defects, and their results are available in Ciemniewska and Jurkiewicz (2007).

Table 30 summarizes the characteristics of each case study. 
Table 30 - Characteristics of the Case Studies

\begin{tabular}{|l|c|c|c|c|c|}
\hline & $\begin{array}{c}\text { Broker System } \\
\text { (6 scenarios) }\end{array}$ & $\begin{array}{c}\text { ATM System } \\
\text { (5 scenarios) }\end{array}$ & $\begin{array}{c}\text { DLibra } \\
\text { (15 scenarios) }\end{array}$ & $\begin{array}{c}\text { Mobile News } \\
\text { (15 scenarios) }\end{array}$ & $\begin{array}{c}\text { Total } \\
\text { (41 scenarios) }\end{array}$ \\
\hline Num. of episodes & 32 & 33 & 80 & 89 & 234 \\
\hline Num. of exceptions & 9 & 5 & 26 & 5 & 45 \\
\hline $\begin{array}{l}\text { Num. of Pre-condition/ } \\
\text { Condition/Cause/Constraint }\end{array}$ & 15 & 8 & 33 & 0 & 56 \\
\hline Num. of Post-condition & 2 & 9 & 0 & 0 & 11 \\
\hline \multicolumn{1}{|c|}{ Total } & $\mathbf{5 8}$ & $\mathbf{5 5}$ & $\mathbf{1 3 9}$ & $\mathbf{9 4}$ & $\mathbf{3 4 6}$ \\
\hline
\end{tabular}

6.1.5.

Subjects

In order to create a referential baseline solution for each project's case study, we need to construct or validate existing material on the analysis results of each of the four projects, which the respective SRSs were translated to our scenario language.

A referential baseline solution lists the defects contained within scenarios or a set of scenarios, which will act as the basis for the evaluation of our analysis approach. A defect in a baseline solution is described using the following format: $<$ Property $>$ - <Type Defect> : <Detail>, where "Property" is the quality negatively impacted by the defect, "Type Defect" is the classification of the defect, and "Detail" gives a description of the defect for fixing. These project's scenarios and baseline solutions can be found in the Appendix 1.

Thus, to start the evaluation of the project's case studies, we counted on five senior Requirements Engineers as volunteers. These engineers are master and Ph.D Computer Science students at PUC-RIO, 30-40 years old, and they have been working in industry for the last 10 years. Particularly, they have been working with use cases for at least 5 years and with scenarios for at least 2 years.

We applied a questionnaire to collect the experience of these volunteers, which showed that they had similar background; for instance, $100 \%$ of them had knowledge about the syntax of Use Cases (Cockburn, 2001) or Scenarios (Leite et al, 2000), and $70 \%$ had some knowledge about requirements analysis or inspection. In order to build a shared understanding on their tasks, they received training on our proposed analysis proposal, as well as on the scenario language used in this thesis. These volunteers will construct a referential baseline solution for each project's case study. This task is based on the existing preliminary analysis results of each case study. 


\section{2. Referential Baseline Solution}

Whereas the analysis with case studies is comparative in nature, we need to contrast the results obtained with our automated approach (implemented in the $C \& L-L u a)$ with another one. To keep experimental biases at a minimal level, a valid basis for assessing the analysis results of the case studies must be identified in advance, which act as the baseline in the evaluation.

The sources and results, with the exception of one, of the SRSs analyzed by related work are available. Somé (2010) only makes available the material analyzed. Cox et al. (2004), and Ciemniewska and Jurkiewicz (2007) make available the material analyzed and their results, i.e., the defects detected by their approaches. Most of the defects contained in the related work preliminary analysis results (set of defects introduced and detected by related work) are mainly related to Completeness properties, and a fewer to Unambiguity properties.

We used two strategies to establish the baselines for each selected case study: Construction and Validation.

We construct a baseline solution for the "Online Broker System" case study from available scenarios.

We construct the baseline solutions for the "ATM System", "DLibra" and "Mobile News" case studies, by reviewing and validating the available use cases and preliminary analysis results.

It is not difficult to validate the preliminary analysis results, because the use case language used by related work to write use cases has corresponding components in scenario language, and a sentence is written in a similar basic grammar; such as introduced in previous section.

We detail the process of creation of baseline solutions for each case study in the following Sub-Sections.

\subsection{1.}

\section{Online Broker System (Somé, 2010)}

Somé (2010) makes available the set of scenarios formalized using Petrinets, however, this work does not detect defects in scenarios, and this cannot be directly used as case study. Therefore, we had to carefully define a process that would allow us obtain an objective baseline solution. 
In order to obtain a baseline solution for the "Online Broke System", the subjects (volunteers) manually inspected the scenarios, identifying defects across the scenario specifications. The engineers were allocated $1 \mathrm{~h}$ to perform the analysis of the set of scenarios, each one working separately from the rest. At last, after some discussions, we validated the defects they detected from the scenarios and established a single baseline solution from this case study. The number of defects reported for this case study was larger than we expected.

The obtained baseline solution contains defects that hurt Unambiguity (Insertion of ambiguous words in sentences), Simplicity (action-verb in incorrect tense, missing of the subject or object, sentences containing more than one actionverb), Usefulness (actor does not participate in sentences, too short or too long episodes, subjects not described in actor/resource element), and Uniformity (incorrect format or missing of the main components in sentences) and Noninterferential (simultaneously enabled operations).

\subsection{2.}

\section{ATM system (Cox et al., 2004)}

Cox et al. (2004) make available the scenarios analyzed and the defects introduced into the scenarios to manually evaluate an inspection technique. They conducted an experiment with final year undergraduate computer and software engineering students taking a course in Total Quality Management (TQM), at University of New South Wales, National ICT Australia, Sydney, Australia. They introduced several types of defects into the scenarios, including syntactic and semantic defects. Semantics defects are difficult to detect by an automated tool, because this need to understand the meaning of the scenario.

In order to obtain a baseline solution for the "ATM System", we entrusted the preliminary analysis results of this case study to the selected subjects (two senior Requirements Engineers).

The requirements engineers received the preliminary analysis results provided by Cox et al. (2004). They manually reviewed and validated the preliminary analysis results of the set of scenarios; they also removed semantic defects detected by Cox et al. (2004). Semantic defects are difficult to detect by an automated tool. The following episode sentence contains a semantic defect, and it was removed: 
User selects 'Change PIN'. (Defect: no reference to enter current PIN $R E Q)$.

In this example, an automated tool could not detect the missing of a previous episode.

After reviewing and validating defects in preliminary analysis results, the requirements engineers identified some new defects, mainly related to Unambiguity properties. The obtained baseline solution contain defects that contribute to Implicitly (sentences containing pronouns), Vagueness (sentences containing adjectives or adverbs), Simplicity (Complex sentences, complex nested conditional sentences, missing action-verb in correct tense or missing object), Usefulness (lack of actor or subject in sentences, subjects not described in actor/resource element, too short or long scenarios), Conceptually Soundness (missing action-verb in sentences) and Liveness (never enabled operations) properties.

\subsection{3.}

\section{DLibra and Mobile News}

These projects are detailed in (Ciemniewska and Jurkiewicz, 2007), i.e., the set of scenarios and the common defects introduced in industrial projects are publicized.

In order to obtain baseline solutions for the "DLibra" and "Mobile News" case studies, we entrusted the preliminary analysis results of this case studies to the selected subjects (two senior Requirements Engineers). They mapped the ten types of defects detected by the approach proposed by Ciemniewska and Jurkiewicz (2007) into defects that contribute to Multiplicity, Simplicity, Usefulness, Conceptually Soundness, Uniformity and Uniqueness properties of our approach.

The requirements engineers received the preliminary analysis results provided by Ciemniewska and Jurkiewicz (2007). They manually reviewed and validated the preliminary analysis results of the set of scenarios; they also detected defects which do not occur in the referential specification, or are incorrectly classified by Ciemniewska and Jurkiewicz (2007). The following episode sentences contain examples of incorrectly classified defects in the baseline provided by Ciemniewska and Jurkiewicz (2007):

User may sort clients. (Defect: depends on some condition). 
User chooses a news group from the 'Today' menu. (Defect: This step is not performed by an actor).

In these examples, these defects are classified incorrectly. In the first sentence, it does not describe a conditional step, however it contains a weak term, then, it is a weak sentence. In the second sentence, it is performed by an actor, then, the defect does not occur.

After reviewing and validating defects in preliminary analysis results, the requirements engineers identified some new defects, mainly related to Unambiguity properties. The obtained baseline solution contain defects that contribute to Multiplicity (Complex sentences containing more than one sentences), Weakness (sentences containing 'may' word), Simplicity (Complex sentences, complex nested conditional sentences, missing action-verb or missing object), Usefulness (lack of actor or subject in sentences, too short or long scenarios, correct step numbering between episodes and exceptions), Conceptually Soundness (missing action-verb in sentences) and Uniformity (Incomplete exceptions) properties.

\subsection{4.}

\section{Summary of Baselines}

Table 31 summarizes the baseline solution for each case study, and lists the number of defects by the quality negatively impacted.

Table 31 - Summary of the Baseline for the Case Studies

\begin{tabular}{|l|c|c|c|c|c|}
\hline & Broker System & ATM System & DLibra & Mobile News & Total \\
\hline Unambiguity & 6 & 9 & 35 & 54 & 104 \\
\hline Atomicity & & & 1 & & 1 \\
\hline Simplicity & 7 & 7 & 35 & 26 & 75 \\
\hline Uniformity & & 2 & & 3 & 5 \\
\hline Usefulness & 6 & 2 & & 3 & 11 \\
\hline Conceptually Soundness & 3 & & & & 3 \\
\hline Integrity & & & & & \\
\hline Coherency & & & & & \\
\hline Uniqueness & 4 & & & & 4 \\
\hline Non-interferential & 2 & & & & 2 \\
\hline Boundedness & & & & & \\
\hline Reversibility & & & & & \\
\hline Liveness & & 2 & & & 2 \\
\hline \multicolumn{1}{|c|}{ Total } & $\mathbf{2 8}$ & $\mathbf{2 2}$ & $\mathbf{7 1}$ & $\mathbf{8 6}$ & $\mathbf{2 0 7}$ \\
\hline
\end{tabular}

In Table 31, it is possible to note that most of the defects are related to Unambiguity (Insertion of ambiguous words in sentences), Simplicity (action-verb in incorrect tense, missing of the subject or object, sentences containing more than 
one action-verb), Usefulness (actor does not participate in sentences, too short or too long episodes, subjects not described in actor/resource element), and Uniformity (incorrect format or missing of the main components in episodes or exceptions).

We put the set of scenarios and baseline solutions of each case study in Appendix 1.

\section{3.}

\section{Evaluation}

After baseline solutions for each case study were established by volunteers, we evaluated our scenario analysis approach implemented in the C\&L - Lua tool by carrying out the following steps:

- We chose the case studies as the input data for the evaluation (set of scenarios);

- We apply the C\&L - Lua to detect defects contained in scenarios of the four case studies;

- We compare the results automatically obtained with the C\&L - Lua by looking at the baselines;

- We measure the accuracy of the analysis results using the evaluation metrics (recall and precision);

- We use these measures to answer the response variables that help to corroborate the hypothesis.

\subsection{1.}

\section{Time Analysis}

According to Alchimowicz (2011), one of the main characteristics of a tool being developed is its efficiency. Not only developers are interested in this metric, but also for the users, e.g. when requirements engineers have to choose between two tools which give the same results, they would choose the one which is more efficient.

During the evaluation of the case studies, time statistics were gathered to asses the performance of the developed solution to automate the proposed analysis approach.

In the evaluation it was assumed that case study length is the number of sentences (Number of episodes + exceptions + pre-conditions + conditions + 
causes + constraints + post-conditions) the set of scenarios is composed of. From the results it can be observed that the analysis can be performed without delays, which would be noticeable for the user. Only processing long case studies (containing from 94 to 139 sentences) takes significant amount of time (as it can be observed at charts in Figure 68). Moreover, according to Somé (2010), because scenarios describes requirements artifacts, the number of scenarios and sentences in projects is typically limited.

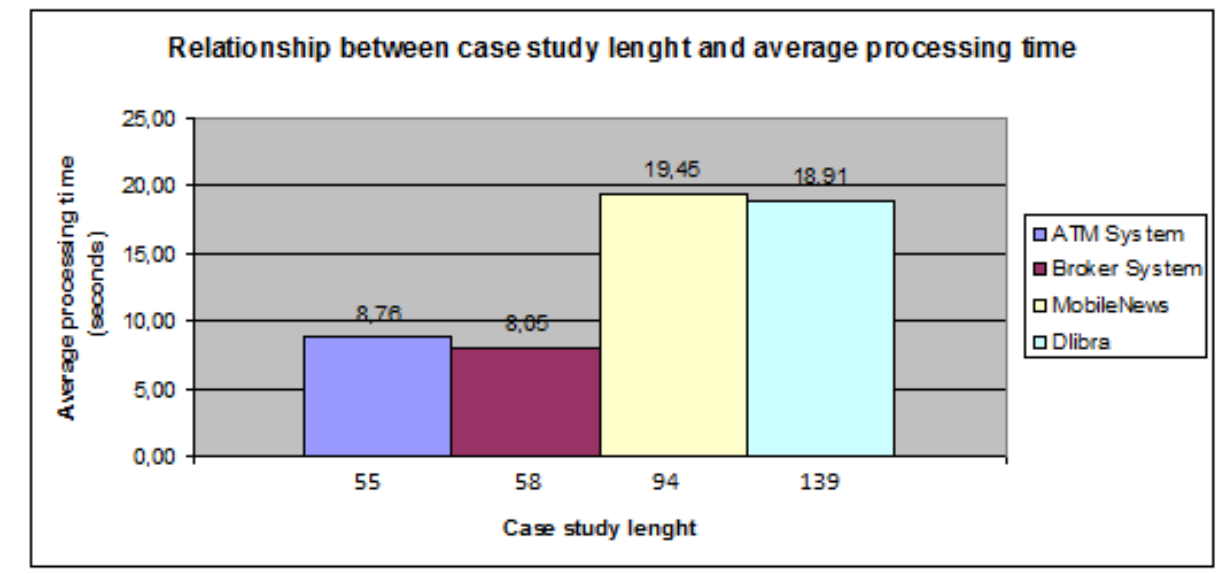

Figure 68 - Relation between case study length and average processing time.

Mobile News (length $=94)$ case study took more time than DLibra (length = 139) because the length (number of words) of the sentences contained in scenarios of Mobile News is larger than DLibra; so that, POS tagging process of the NLP tool took more time.

\subsection{2.}

\section{Analysis Results}

The referential scenario specifications were analyzed by the proposed analysis approach in order to find the defects described for each one of the properties related to Unambiguity, Completeness and Consistency qualities described in Chapter 3.

We apply the $\boldsymbol{C} \boldsymbol{\&} \boldsymbol{L}-\boldsymbol{L u a}$ tool introduced in Chapter 5 to the four scenario sets. The resulting quality analysis shows promising results that indicate high potential for successful further improvements.

Appendix 1 summarizes the data collected from the execution of $\boldsymbol{C} \boldsymbol{\&} \boldsymbol{L}-$ Lua on each of the case studies by comparing the output of our analysis approach against the corresponding baselines. The obtained results ( $\boldsymbol{T P}, \boldsymbol{F P}$ and $\boldsymbol{F N})$ were used to measure the accuracy of the $\boldsymbol{C} \boldsymbol{\&} \boldsymbol{L}-\boldsymbol{L u} \boldsymbol{a}$ tool. For each case study, all of 
the processed scenarios violate one or more quality properties that the $\boldsymbol{C} \boldsymbol{\&} \boldsymbol{L}-\boldsymbol{L u a}$ can detect.

In the following sub sections are shown the results achieved by the $\boldsymbol{C} \boldsymbol{\&} \boldsymbol{L}-$ Lua on each case study, which indicate the Recall and Precision of the approach to detect defects that hurt the main quality properties of scenarios.

\subsubsection{1.}

\section{Results of Unambiguity Analysis}

As it can be seen from the results in Table 32, unambiguity defects were detected with the $100 \%$ recall and high precision. Only the precision for multiplicity defects is under $100 \%$ which results from the natural language ambiguities described in Chapter 3.

C\&L - Lua achieved a Precision above $75 \%$. When this is applied to detect defects related to Multiplicity, the precision is lower (75\%). Multiplicity defects are difficult to detect because a sentence can describe several attributes for the same entity, and this fact can be understand by an automated tool as attributes for different entities. For example, in the "Broker System" case study, the following episode sentence is incorrectly recovered (false positive) as containing a multiplicity defect (defect indicator: "and"):

"Broker System asks for Customer name, date of birth and address".

In this example, the $\boldsymbol{C} \boldsymbol{\&} \boldsymbol{L}-\boldsymbol{L} \boldsymbol{u} \boldsymbol{a}$ tool understood that name, date of birth and address are attributes of different entities; however, they are attributes of the customer entity.

Table 32 - Analysis of Unambiguity using the C\&L - Lua.

\begin{tabular}{|l|c|c|c|c|c|c|c|c|}
\hline Unambiguity & \multicolumn{2}{|c|}{ Broker System } & \multicolumn{2}{c|}{ ATM System } & \multicolumn{2}{c|}{ DLibra } & \multicolumn{2}{c|}{ MobileNews } \\
\cline { 2 - 9 } Analysis & Recall & Precision & Recall & Precision & Recall & Precision & Recall & Precision \\
\hline Vagueness & 1 & 1 & & & 1 & 1 & 1 & 1 \\
\hline Subjectiveness & & & & & & & & \\
\hline Optionality & & & & & & & & \\
\hline Weakness & & & & & 1 & 1 & & \\
\hline Multiplicity & 1 & 0.75 & 1 & 1 & 1 & 0.86 & 1 & 0.97 \\
\hline Implicitly & 1 & 1 & 1 & 1 & 1 & 1 & 1 & 1 \\
\hline Quantifiability & & & & & 1 & 1 & 1 & 1 \\
\hline \multicolumn{1}{r|}{ Total } & $\mathbf{1}$ & $\mathbf{0 . 8 6}$ & $\mathbf{1}$ & $\mathbf{1}$ & $\mathbf{1}$ & $\mathbf{0 . 9 5}$ & $\mathbf{1}$ & $\mathbf{0 . 9 8}$ \\
\hline
\end{tabular}

Overall, the $\boldsymbol{C} \boldsymbol{\&} \boldsymbol{L}-\boldsymbol{L u} \boldsymbol{a}$ produced reasonable results with perfect recall and above $86 \%$ precision. 


\subsubsection{2. \\ Results of Completeness Analysis}

From the analysis results of Completeness properties (Table 33), one can observe that every analyzed scenario sentence was correctly classified by the $\boldsymbol{C} \boldsymbol{\&} \boldsymbol{L}$ - $\boldsymbol{L} \boldsymbol{u} \boldsymbol{a}$ tool. When it comes to the individual properties Recall results, the $\boldsymbol{C} \boldsymbol{\&} \boldsymbol{L}-$ Lua identified the entire set of defects in 3 of the case studies (Broker System, ATM System and DLibra). This result is reflected by a perfect recall score in the Table 33. In the "MobileNews" case study, the tool obtained $93 \%$ recall.

"MobileNews" results had two false negatives, attributed to two sentences with more than one action-verb that were not uncovered. Such behavior can be explained by the specificity of English. In English some words can be both verbs and nouns (as described in Chapter 5). This leads to a situation when verb can be confused with noun, for instance in the following sentences:

"System finds all users matching deletion criteria and deletes found user accounts"

"System receives the confirmation and displays it"

In these examples, the $\boldsymbol{C} \boldsymbol{\&} \boldsymbol{L}-\boldsymbol{L} \boldsymbol{u} \boldsymbol{a}$ understood that "deletes" and "displays" are nouns (NNS).

Regarding the individual properties Precision results, the $\boldsymbol{C} \boldsymbol{\&} \boldsymbol{L}-\boldsymbol{L u a}$ obtained acceptable results. In Broker System and DLibra, $\boldsymbol{C} \boldsymbol{\&} \boldsymbol{L} \boldsymbol{L} \boldsymbol{u} \boldsymbol{a}$ achieved a precision with $100 \%$. In ATM System and MobileNews, $\boldsymbol{C} \boldsymbol{L} \boldsymbol{L}-\boldsymbol{L} \boldsymbol{u} \boldsymbol{a}$ achieved $75 \%$ and $88 \%$ precision. This value means that from the complete set of defects identified, only a quarter corresponded to false positives.

ATM System and MobileNews results had 4 and 3 false positives, caused by the "Parsing" strategy used to identify subjects and action-verbs in sentences (Detailed in Chapter 5). Such behavior is due to the situation when verb can be confused with a noun or adjective, for instance in the following sentences:

System assigns an expiry date and time to each incoming message

User re-enters new PIN.

In these examples, the "parsing" strategy understood that "time" is a verb (VB), and "re-enters" is an adjective (JJ).

A more detailed analysis revealed that this precision loss was also caused by the "Syntactic Similarity" heuristic used to measure the coherency between the title and the goal of a scenario (Detailed in Chapter 5). We noticed that some 
scenario titles and goals are written using synonymous terms (i.e., domain entities or actions can be different names or synonymous). Consequently, the detection problem can be attributed to the syntactic analysis strategy used by our approach, which is not improved by a semantic strategy. For instance, this situation was encountered in the following sentences:

\section{TITLE:} WITHDRAW CASH

GOAL: User wants to withdraw money.

In this example, the "cash" and "money" are synonymous, but they are understood as different terms by our approach.

Table 33 - Analysis of Completeness using the C\&L - Lua.

\begin{tabular}{|l|c|c|c|c|c|c|c|c|}
\hline $\begin{array}{l}\text { Unambiguity } \\
\text { Analysis }\end{array}$ & \multicolumn{2}{|c|}{ Broker System } & \multicolumn{2}{c|}{ ATM System } & \multicolumn{2}{c|}{ DLibra } & \multicolumn{2}{c|}{ MobileNews } \\
\cline { 2 - 9 } & Recall & Precision & Recall & Precision & Recall & Precision & Recall & Precision \\
\hline Atomicity & & & & & 1 & 1 & & \\
\hline Simplicity & 1 & 1 & 1 & 0.88 & 1 & 1 & 0.93 & 0.93 \\
\hline Uniformity & & & 1 & 1 & & & 1 & 0.75 \\
\hline Usefulness & 1 & 1 & 1 & 1 & & & 1 & 1 \\
\hline $\begin{array}{l}\text { Conceptually } \\
\text { Soundness }\end{array}$ & 1 & 1 & & & & & & \\
\hline Integrity & & & & & & & & \\
\hline Coherency & & & & & & & & \\
\hline Uniqueness & 1 & 1 & & & & & & \\
\hline \multicolumn{1}{r|}{ Total } & $\mathbf{1}$ & $\mathbf{1}$ & $\mathbf{1}$ & $\mathbf{0 . 7 3}$ & $\mathbf{1}$ & $\mathbf{1}$ & $\mathbf{0 . 9 4}$ & $\mathbf{0 . 9 1}$ \\
\hline
\end{tabular}

Overall, the $\boldsymbol{C} \boldsymbol{\&} \boldsymbol{L}-\boldsymbol{L} \boldsymbol{u a}$ produced reasonable results with above $94 \%$ recall and $73 \%$ precision.

\subsubsection{3. Results of Consistency Analysis}

Reveal the incorrect behavior (dynamic) of a set of scenarios from initial requirements engineering activities is difficult and hard. This problem can be attributed to the informal nature of scenario descriptions, usually written using natural language. Thus, it is difficult to establish a baseline solution to compare with analysis results obtained using an automated approach.

However, the requirements engineers manually inspected the scenarios of "Broker System" and "ATM System" case studies, and they identified defects like Non-interferential and Liveness (Table 31). In short projects, it can be possible identify non-deterministic situations or never executed sentences, without executing or simulating the scenarios using rigorous representations.

As it can be seen from the results in Table 34, consistency defects were detected with the $100 \%$ recall and precision. 
Table 34 - Analysis of Consistency using the C\&L - Lua.

\begin{tabular}{|c|c|c|c|c|c|c|c|c|}
\hline \multirow{2}{*}{$\begin{array}{c}\text { Unambiguity } \\
\text { Analysis }\end{array}$} & \multicolumn{2}{|c|}{ Broker System } & \multicolumn{2}{|c|}{ ATM System } & \multicolumn{2}{|c|}{ DLibra } & \multicolumn{2}{|c|}{ MobileNews } \\
\hline & Recall & Precision & Recall & Precision & Recall & Precision & Recall & Precision \\
\hline Non-interferential & 1 & 1 & & & & & & \\
\hline Boundedness & & & & & & & & \\
\hline Reversibility & & & & & & & & \\
\hline Liveness & & & 1 & 1 & & & & \\
\hline Total & 1 & 1 & 1 & 1 & & & & \\
\hline
\end{tabular}

When facing large projects, the number of scenarios could be unmanageable and the requirements engineers have difficulties to manually identify inconsistencies from the behavior of scenarios, mainly because the relationships among several scenarios of the project can not be explicit.

With such situations on mind, the $\boldsymbol{C} \boldsymbol{\&} \boldsymbol{L}-\boldsymbol{L u a}$ generates warning or information messages instead of error, to indicate possible behavioral defects. $\boldsymbol{C} \boldsymbol{\&} \boldsymbol{L}-\boldsymbol{L u} \boldsymbol{a}$ can be applied to detect such defects as non-deterministic situations, deadlocks and never enabled operations. That is, it is sufficient to translate related scenarios (identified by our approach, such as demonstrated in Chapter 4) into a consistent Integrated Petri-Net and to perform simulation and reachability analysis over the Petri-Net.

$\boldsymbol{C} \boldsymbol{\&} \boldsymbol{L}-\boldsymbol{L} \boldsymbol{u a}$ generates warning messages to indicate possible deadlock when two or more scenarios are executed concurrently. Such behavior is due to simultaneously executed scenarios. For instance in the "Broker System", the suppliers are executed concurrently due to common pre-conditions, such behavior can produce a potential deadlock among then. Figure 69 shows the warning message produced by the $\boldsymbol{C} \boldsymbol{\&} \boldsymbol{L}-\boldsymbol{L u} \boldsymbol{a}$ to indicate a possible path to deadlock when suppliers' scenarios are invoked from the main scenario "Submit Order".

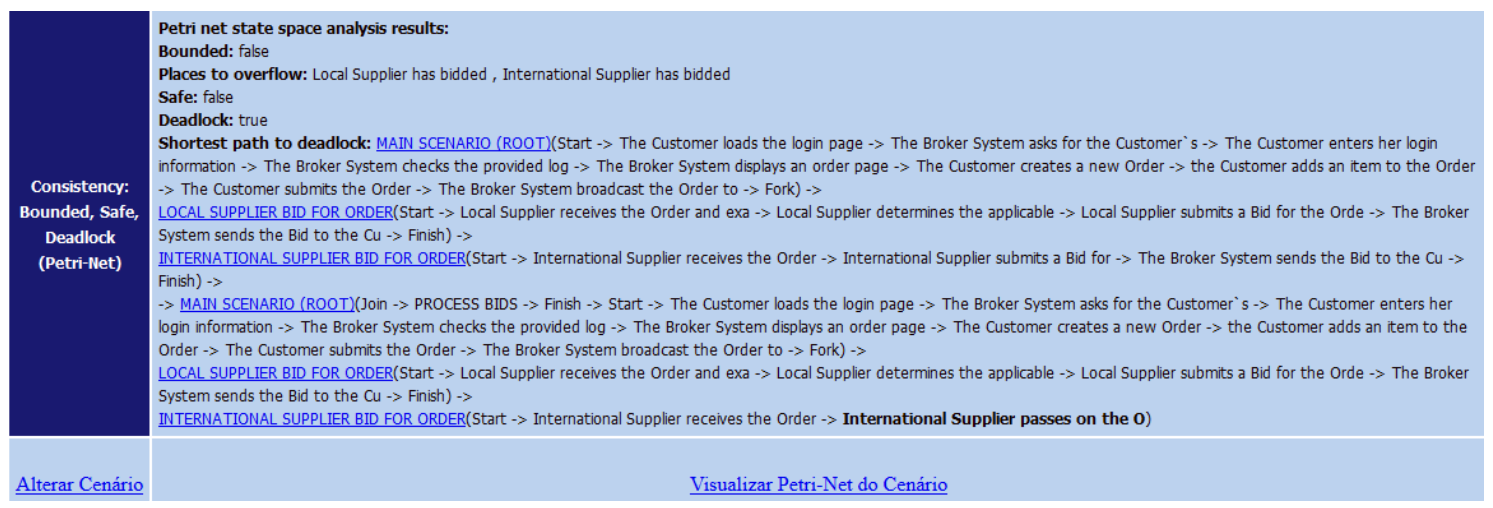

Figure 69 - Consistency Analysis Using Petri-Nets in "Broker System".

"MobileNews" analysis results recovered Liveness defects, attributed to 4 exceptions which are never enabled for execution. Such behavior can be detected 
by simulating the Petri-Net and detecting never enabled transitions. Figure 70 shows a never enabled exception from "Delete a User Group" scenario.

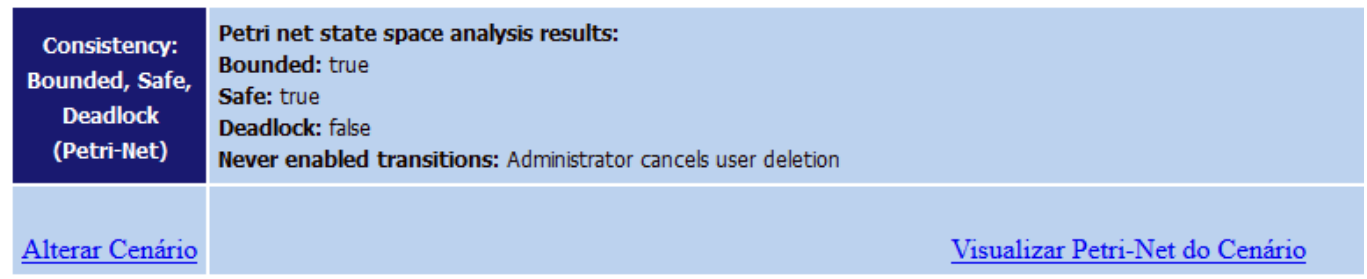

Figure 70 - Consistency Analysis Using Petri-Nets in "Mobile News".

\subsubsection{4}

\section{Results of Correctness Analysis}

Considering that Correctness is positively contributed by Unambiguity, Completeness and Consistency qualities; we can aggregate the quality results produced by related properties.

Overall, the automated identification of defects produced reasonable results. Aggregated values of the above accuracy-metrics are as in Table 35:

Table 35 - Analysis of Correctness using the C\&L - Lua.

\begin{tabular}{|r|c|c|c|c|}
\hline & Broker System & ATM System & DLibra & Mobile News \\
Correctness Analysis & & & & \\
\hline Recall & 1 & 1 & 1 & 0.97 \\
\hline Precision & 0.96 & 0.91 & 0.98 & 0.95 \\
\hline
\end{tabular}

$\boldsymbol{C} \boldsymbol{\&} \boldsymbol{L}-\boldsymbol{L} \boldsymbol{u a}$ detects the total amount of scenarios with defects with above $91 \%$ precision and $97 \%$ recall.

\section{4 . Interpretation}

Four case studies have been carried out to evaluate the accuracy of the proposed analysis approach. These set of scenarios have been evaluated with respect to Information Retrieval metrics. We evaluated the degree of accuracy of results produced by the developed tool (C\&L-Lua) with respect to reference solutions elaborated by expert Requirements Engineers.

The general hypothesis was verified: "the proposed automated analysis approach should help to identify and show a great deal of defects from a set of scenario specifications, and furthermore take less time than what it would take to Requirements engineers". The proposed solution detects defects in scenarios in an acceptable response time, and with close to $100 \%$ recall and above $83 \%$ precision, inline with Berry's notion (Berry 2012). 
The results obtained with the proposed approach implemented in $C \& L-$ Lua were precise, making only a few mistakes in the detection process. The overall precision was quite high ( $93 \%$ precision), exceeding our expectations. Three out of 4 case studies obtained the maximum recall, which means that, for those defects correctly identified, our approach was able to provide the right advice every time.

The analysis of the evaluation allows to state that the developed methods and heuristics of automatic defects detection are reliable and can significantly improve the quality of the scenario descriptions. Although these set of scenarios describe some abstract systems, they show the typical defects of the industrial requirements specifications, such as demonstrated in (Alchimowicz, 2011).

Finally, case studies show that the developed referential scenario specifications can be used in different ways by both researchers and analysts.

\subsubsection{Accuracy of the Petri-Net Generator}

We generate one Petri-Net for each scenario; the accuracy of the method to transform a scenario into a Petri-Net is measured by the accuracy of the control flow information of the generated Petri-Net. To be specific, we check (1) whether the nodes (places and transitions) and arcs are correctly generated and linked in the Petri-Net and (2) whether the input places (pre-conditions, conditions, constraints or causes) are correctly associated with the corresponding transitions (episode sentence or exception solution) in the Petri-Net.

According to Feasibility property of Completeness (Chapter 3), it must be possible to perform each operation described in a scenario and each internal/external condition must not be violated. $C \& L-L u a$ evaluates this property by: (1) deriving a Petri-Net for each scenario, and (2) verifying that exist at least a path from the first place (Initial state) to every Petri-Net node. The results obtained shown that all scenarios were correctly translated to Petri-Nets, i.e., they are feasible.

Appendix 1 details the number of Petri-Net input places, transitions and output places generated for each Scenario pre-condition, condition, constraint, cause, episode sentence, exception solution and post-condition, accordingly. 


\subsubsection{Considerations about Scalability}

The case studies considered in this Chapter, involve projects that specify between 5 and 15 scenarios with different degree of complexity, i.e., every scenario describes between 3 and 12 episodes, between 1 and 5 exceptions, and 1 concurrency construct (\#<episode series>\#). However, in order to evaluate the scalability of our approach it will be necessary to verify the accuracy and response time using larger projects.

In the literature, it is difficult to find publicly available referential specifications to evaluate defect detection approaches. Only, Alchimowicz et al. (2011) make available a referential use case specification containing typical defects in industrial projects. Based on the use cases stored in UCDB (2015), they developed a Referential Specification that has a near-typical profile, that means that its properties is more or less what you can expect to find in real projects. They make available this referential specification to be used for benchmarking tools for use-case analysis.

We used the "Admission System" referential specification (Alchimowicz et al., 2011) to evaluate the scalability of the $C \& L-L u a$. However, they do not make available the defects introduced in the use cases to compare with the results obtained by our approach. "Admission System" case study is a system, which enables candidates to apply for the studies through the Internet.

First, I translated the 34 use cases into scenario descriptions; this task is not difficult because use case components (pre-condition, post-condition, steps and extensions) have corresponding components in scenario language (pre-condition, post-condition, episodes and exceptions). Table $\mathbf{3 6}$ shows the characteristics and length of this case study.

Table 36 - Characteristics of the Admission System Case Study

\begin{tabular}{|l|c|}
\hline & Admission System (34 scenarios) \\
\hline Num. of episodes & 161 \\
\hline Num. of exceptions & 75 \\
\hline Num. of Pre-conditions/Conditions/Causes & 79 \\
\hline Num. of Post-conditions & \\
\hline \multicolumn{1}{|c|}{ Total } & $\mathbf{3 1 5}$ \\
\hline
\end{tabular}

From the evaluation results, we observed that the analysis can be performed without delays. The processing took 1 minute and 35 seconds, and detected defects that hurt Unambiguity (Insertion of ambiguous words in sentences), 
Simplicity (action-verb in incorrect tense, missing of the subject or object, sentences containing more than one action-verb), Usefulness (actor does not participate in sentences, too short or too long episodes), Uniqueness (scenarios enabled by the same pre-conditions) and Liveness (never enabled exceptions).

This shows that the developed tool for defects detection handled the load, therefore scaling to larger projects may not be an issue because of the generally polynomial complexity of Petri-Net transformation, Petri-Net integration and NLP Syntactic Analysis heuristics implemented in the $C \& L-L u a$. However, more studies should be carried out.

Other important strategy to improve the scalability of our analysis approach is the MULTI-STEP BOTTOM-UP consistency analysis approach. This strategy reduces the state explosion of the reachability graph for large and complex PetriNets, because it analyses a large system in a compositional way.

\section{5 .}

\section{Threats to Validity}

In order to reduce the subjectivity of the results obtained in the manual analysis (baseline), we verified that the requirements engineers had similar degrees of background about scenarios or scenarios inspection techniques, by means of a questionnaire. Therefore, more studies should be carried out by requirements engineers with non-homologous profiles.

In order to translate use case descriptions (reported in the related work) into scenario descriptions (proposed in this thesis), manual effort are needed. In this translation process some issues can be introduced, however this is not considered harmful. This task is not difficult because use case components (pre-condition, post-condition, steps and extensions) have corresponding components in scenario language (pre-condition, post-condition, episodes and exceptions), and step/alternative sentences are written in a similar basic grammar (a basic sentence is composed of a 3-tuple subject-verb-object).

\section{6.}

\section{Conclusion}

In order to corroborate the hypothesis, we used statistical metrics to answer the response variables. Thus, measures of the analysis results are considered reliable. 
In order to generalize the results obtained in this evaluation, we used SRSs with different degree of complexity, size, domain, common defects reported in the industry. However more empirical experiment and detailed analysis of proposed heuristics are advisable. 


\section{7 \\ Conclusion}

Natural language based requirements specification techniques, like the scenario language explored in this work, helps users and developers to improve the quality at early activities of software development process. As such, the analysis of software requirements specifications described as scenario representations improves the quality of the product from the initial stages of software production, contributing to the reduction of failures and the reduction of maintenance costs after the final product was developed.

In this context, it is relevant to develop techniques that enable the automated analysis of these scenarios, so that defects in early requirements artifacts may be identified in way that is more efficient.

Frequently, scenario's representations are normally informal or semi-formal and, in these cases, they cannot be used for further automated analysis. In order to identify defects within scenarios, it is necessary: (1) to review the scenarios using formal inspection techniques; (2) to pre-process the scenario descriptions to reduce the ambiguity and analyze them using Natural Language Processing strategies; or (3) to translate the scenarios from informal to formal representations, like Petri Nets, and simulate the behavior of them to identify consistency defects.

The assessment of quality properties in requirements artifacts has long been investigated in requirements engineering. These are complex concepts, that demands the fulfillment of many others characteristics in order to be achieved.

The main aim of this thesis was to develop an automated approach for detecting defects in scenario specifications to support the analysis of the SRSs. This aim has been achieved by: (1) modeling and organizing the properties related to scenario's quality; (2) distinguishing the defect indicators that hurt these quality properties; (3) proposing operationalizations or heuristics to search these defect indicators; (4) proposing heuristics for finding non-explicit relationships among scenarios, and improve the analysis results; (5) investigating Natural Language Processing techniques for automatic detection of syntactic defects; and (6) 
investigating rigorous mechanisms to translate scenarios into Petri-Nets and simulate the behavior of a set of related scenarios.

In this thesis, we introduced a novel perception of Correctness and its complex relationships with Unambiguity, Completeness and Consistency describing it as a quality that should be satisficed by contributions of related qualities or properties. We also shown how the properties of: (1) Vagueness, Subjectiveness, Optionality, Multiplicity, Quantifiability, Readabiity, Minimality, Weakness and Implicitly contribute to Unambiguity; (2) Atomicity, Simplicity, Uniformity, Usefulness, Conceptually Soundness, Integrity, Coherency and Uniqueness contribute to Completeness; (3) Non-interferential, Boundedness, Reversibility and Liveness contribute to Consistency; and (4) Unambiguity, Completeness and Consistency contribute to Correctness. It means that addressing these properties, we are contributing to requirements Unambiguity, Completeness, Consistency, and consequently to Correctness.

As scenarios are written in natural language, we investigated NLP strategies to develop mechanisms of automatic detection of structural or syntactic defects, i.e., these mechanisms verify that scenario sentences are composed of basic attributes like Subject, Action-Verb and Object.

In order to detect defects due to behavioral properties of a set of related scenarios, we proposed an automated strategy to simulate and detect defects from the execution; this strategy is based on the transformation of a set of related scenarios into a whole consistent Petri-Net model. From this transformation, it was possible to: (1) Define criteria to verify Feasibility, i.e., verify that is possible derive an initial system design from a set of related scenarios; (2) analyze behavioral properties (like reachability, boundness and liveness) of equivalent Petri-Nets; and (3) support traceability, detecting the defects in Petri-Net models and indicating the defects within scenarios or in their relationships.

To increase the practical meaning of the proposed methods and heuristics, a prototype solution was developed. The prototype solution was designed and implemented as a set of modules of the $\boldsymbol{C} \boldsymbol{\&} \boldsymbol{L}-\boldsymbol{L u} \boldsymbol{a}$ (Almentero, 2009). This tool assists the requirements engineers during the requirements modeling and analysis phases.

To assess the quality of the results achieved by the C\&L - Lua, five case studies were carried out on a set of 75 scenarios with 661 sentences altogether. 
The analysis of this evaluation shows that the developed solution is reliable and can improve the requirements quality.

\section{1.}

\section{Comparison with Related Work}

Many researches have shown the importance to address the problem of finding defects in early software requirements artifacts written using Natural Language. Some approaches propose inspection techniques with quality models to evaluate properties of scenario specifications (Anda and Sjoberg, 2002; Leite et al., 2005; Phalp et al., 2007); usually these approaches are manually applied by requirements inspectors. Other approaches, in order to benefit from automated scenarios analysis, propose the use of Natural Language Processing strategies to analyze structural defects in scenario sentences (Ciemniewska and Jurkiewicz, 2007; Liu et al., 2014). Some research focused on developing the formal semantics for scenario analysis (Hsia et al., 1994; Cheung et al., 2006). Others are focusing on developing techniques to translate scenarios into executable models and detect inconsistencies among scenarios (Lee et al., 1998; Lee et al., 2001; Sinnig et al., 2009; Zhao and Duan, 2009; Somé, 2010).

In (Anda and Sjoberg, 2002; Leite et al., 2005; Phalp et al., 2007) are presented approaches that address the problem of finding defects in scenarios documents with the aid of quality models and inspection techniques. These approaches assess the quality of the documents and to provide hints to potential ambiguities, incompleteness and inconsistencies within the use case descriptions. They are manually performed, and they do not provide insights for further automation. Only Leite et al. (2005) provides some feasible heuristics for finding defects in scenarios.

In (Ciemniewska and Jurkiewicz, 2007; Liu et al., 2014) are proposed approaches to identify syntactic defects in use case documents (semi) automatically with the aid of Natural Language Processing (NLP) techniques. These approaches extract relevant information from scenario sentences and verify that are correctly written. They use phrase parsing strategy to identify the Subject, Action-Verb and Object of a use case sentence; and appoint incompleteness or inconsistencies in use case descriptions. However, in order to improve the 
accuracy of the phrase parser, manual effort is needed to train the parser by providing annotated data.

In (Lee et al., 1998; Lee et al., 2001; Sinnig et al., 2009; Zhao and Duan, 2009; Somé, 2010) are proposed systematic procedures to convert use case descriptions into Petri-Nets (or their variations) or Labeled Transition Systems LTS (Sinnig et al., 2009), allowing the analysis of use cases. To facilitate the transformation, use cases are described using a semi-formal syntax. Reachability analysis techniques are used to evaluate completeness and consistency properties in equivalent Petri-Nets. However, in most of cases, intermediate models are created (Lee et al., 1998; Zhao and Duan, 2009), relationships among use cases are not considered, defects detected in Petri-Nets are not traced to scenarios, and they are not automated. Moreover, only Lee et al. (1998) manages the state explosion issue, a problem of Reachability Analysis.

More details of each one of these approaches are presented in Related Work section of Chapter 2.

In contrast, our approach: (1) defines the properties that contribute to scenarios quality, and describes defect indicators that contribute to these properties; (2) uses a semi-structured natural language to write scenarios; (3) presents heuristics for finding non-explicit relationships among scenarios; (4); takes into consideration the results achieved by requirement statements and user story analysis techniques in finding ambiguity indicators; (5) investigates NLP techniques and linguistic characteristics in order to improve the accuracy of parsing strategy; (6) implements automated transformation rules from scenarios into Petri-Nets; (7) preserves the original properties of scenarios when they are translated; (8) identifies potential concurrency defects due to non-sequential relationships; (9) manages the state explosion in Petri-Net analysis; and (10) demonstrates the feasibility of our proposal by implementing the proposed heuristics and methods in a prototype tool. Finally, no additional manual effort is needed for analysis. Table 37 compares our approach with related approaches. 
Table 37 - Comparing SRS Analysis Techniques

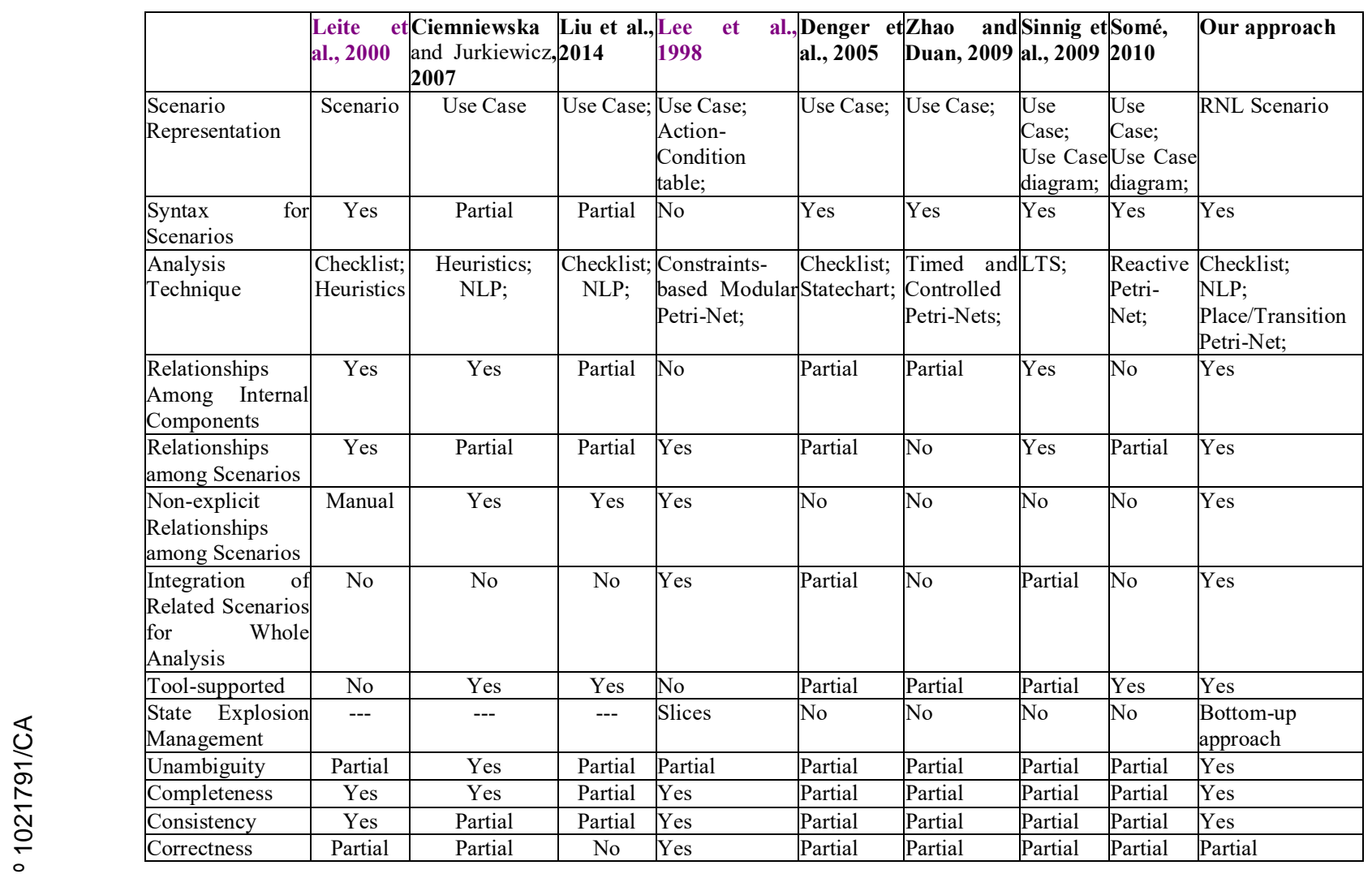

\section{2.}

\section{Contribution}

The proposed approach offers four major contributions:

- An automated scenarios analysis architecture: We presented an architecture for scenarios analysis, composed by: 1) Algorithms to transform scenario descriptions into Petri-Net models, 2) Criteria to analyze the unambiguity, completeness, consistency and correctness of scenario descriptions based on analysis of structural and behavioral properties of Petri-Nets, 3) Criteria to interpret the results obtained from Petri-Nets analysis and to allow requirements engineers reduce fault locating time significantly in requirements at early activities of development. Initial results of this approach have been published in (Sarmiento et al. 2015a; Sarmiento et al. 2014e).

- A restricted-form of natural language for scenarios: We presented semi-structured linguistic patterns for writing scenario elements, such as 
episodes, exceptions, constraints and concurrency; it reduces the ambiguity in natural language scenario descriptions; we also presented formal heuristics for finding non-explicit relationships among scenarios. Initial result has been published in (Sarmiento et al. 2015b).

- A Quality Model for Scenarios: The definition of a reusable Quality Model for Scenarios, which describes the potential Defect Indicators that contribute to properties of the Quality Model from previous related works about requirements statements, user story, use case and scenarios languages. Initial result has been published in (Sarmiento et al. 2015c).

- Modularity: A systematic procedure to synthesize a system design from the resulting Petri-Nets of related scenarios, which manages the State Explosion Issue. State explosion issue is a serious problem when applying Petri-Net analysis to large systems. A contribution of this thesis is a MULTI-STEP BOTTOM-UP analysis approach to manage this problem. Initial result has been published in (Sarmiento et al. 2015a).

Our scenario analysis approach can be applied to a set of scenarios of a project or individual scenarios. When it is applied to a specific scenario, the analysis is carried out on the selected scenario and its related scenarios. So that, the impact among related scenarios are analyzed and defects into the relationships are identified. Therefore, our analysis approach can be used with incremental software development strategies and it also contributes to better understand scenarios evolution and their impacts.

\section{3.}

\section{Limitation}

The transformation procedure from scenarios into Petri-Nets works well if a requirements engineer can properly write scenarios using the syntax and semantic rules described in this thesis, i.e. following the linguistic patterns and putting the correct markers (IF THEN, Constraint, and so on) on sentences, It is our assumption that the use of Restricted-form of Natural Language scenarios is well accepted by the most stakeholders in RE process, and it is amenable to automated processing.

The scalability of Petri-Net model and the state explosion of the generated reachability graph are limitations, however these limitations are overcame because 
the proposed analysis approach is scalable; the analysis of a large and complex system can be performed in a compositional way, i.e., a MULTI-STEP BOTTOM-UP analysis approach.

The Part-Of-Speech tagger is written in JavaScript programming language, thus the parser memory usage expands roughly with the square of the sentence length. Although, we considered case studies with typical defects and length of industrial scenarios, additional experiments with the requirements taken from the industry could be helpful.

\section{4.}

\section{Future Work}

The C\&L prototype tool has been used and evolved by the PUC - Rio requirements engineering group. Methods for model transformation and syntactic analysis using NLP are being improved. Their results are positive and therefore its evolution continues.

In the future, we plan investigating other properties related to the main qualities considered; and instantiate the Quality Model used in this thesis to use case language proposed by Cockburn (2001) or its variations.

In a future research, we will explore to enrich our approach by considering semantic analysis. The WordNet (2015) database can be inspected to provide additional information like synonymous of the "Nouns" and "Verbs" of POS tagging phase; and improve the accuracy of the parsing strategy and syntactic similarity heuristic.

Moreover, as a future research direction, we intend to extend the approach to analyze scenarios written in other languages, e.g. Portuguese.

Other future research plan will consider investigating strategies, which automatically traverse the Petri-Net model and its reachability graph to generate test scenarios based on path analysis strategies. This strategy will take into account interactions by "shared resources" or "message passing". Initial results of this approach have been published in (Sarmiento et al. 2015d; Sarmiento et al. 2014e). 


\section{References}

ALCHIMOWICZ, B.; JURKIEWICZ, J.; NAWROCKI, J.; OCHODEK, M. Towards use-cases benchmark. In: Software engineering techniques. Lecture notes in computer science, Springer-Verlag, v. 4980, Heidelberg, p 20-33, 2011.

ALMENTERO, E.; LEITE, J. C. S. P.; LUCENA, C. Towards Software Modularization from Requirements, In: ACM symposium on Applied Computing, 2014. Proceedings of ACM symposium on Applied Computing, 2014.

ALMENTERO, E. Re-engenharia do software C\&L para plataforma LuaKepler utilizando princípios de transparência. Master Thesis, PUC-Rio, Brazil, 2009.

AL-OTAIBY, T. N.; ALSHERIF, M.; BOND, W. P. Toward software requirements modularization using hierarchical clustering techniques. In: Annual Southeast Regional Conference, 2005. Proceedings of the 43rd annual southeast regional conference, 2005, p. 223-228.

ANDA, B.; SJØBERG, D. I. Towards an inspection technique for use case models. In: International conference on Software engineering and knowledge engineering, 2002. Proceedings of the 14th international conference on Software engineering and knowledge engineering, 2002, p. 127-134.

ANDA, B.; HANSEN, K.; SAND, G. An investigation of use case quality in a large safety-critical software development project. Information and Software Technology, v. 51, n. 12, p. 1699-1711, 2009.

ANDERSSON, M.; BERGSTRAND, J. Formalizing Use Cases with Message Sequence Charts. 1995. Master's thesis, Lund Inst. of Technology.

ALEXANDER, I. F.; MAIDEN, N. Scenarios, stories, use cases: through the systems development life-cycle. John Wiley \& Sons, 2005.

ARORA, C.; SABETZADEH, M.; BRIAND, L.; ZIMMER, F. Automated checking of conformance to requirements templates using natural language processing. IEEE TSE, 2015.

BANSAL, S. Text data cleaning steps python. 2014. Available at: http://www.analyticsvidhya.com/blog/2014/11/text-data-cleaning-stepspython/

BASILI, V.; ROMBACH, H. D. "The TAME Project: Towards ImprovementOriented Software Environments". IEEE Trans. on Software Engineering, v. 14, n. 6, pp. $758-773,1988$. 
BERNSTEIN, L.; YUHAS, C. M. Trustworthy Systems Through Quantitative Software Engineering. Wiley-IEEE Computer Society Press, 2005.

BERRY, D.; GACITUA, R.; SAWYER, P.; TJONG, S. "The Case for Dumb Requirements Engineering Tools". Requirements Engineering: Foundation for Software Quality, Springer, v. 7195, p. 211-217, 2012.

BOEHM, B. W. "Guidelines for verifying and validating software requirements and design specifications". In: European Conf. Applied Information Technology, 1979. Proceedings of European Conf. Applied Information Technology, 1979, p. 711-719.

BOEHM, B.; BASILI, V. R. Software Defect Reduction Top 10 List. Computer, v. 34, n. 1, p.135-137, 2001.

CABRAL, G.; SAMPAIO, A. Formal specification generation from requirement documents. In: Brazilian Symposium on Formal Methods (SBMF), 2006. Proceedings of Brazilian Symposium on Formal Methods (SBMF), 2006, p. 217-232.

CAMBRIDGE.

2015.

Available

at:

http://dictionary.cambridge.org/grammar/british-grammar/verbs-types

C\&L, Scenarios \& Lexicons. 2015. Available at: http://pes.inf.puc-rio.br/cel.

CHEUNG, K. S.; CHEUNG, T. Y.; CHOW, K. O. A petri-net-based synthesis methodology for use-case-driven system design. J. Syst. Softw. v. 79, n. 6, p. 772-790, 2006.

CHUNG, L.; NIXON, B.A.; YU, E.; MYLOPOULOS, J. Non-Functional Requirements in Software Engineering. Boston: Kluwer Academic Publishers, 2000.

COHN, M. User Stories Applied: for Agile Software Development. Redwood City: Addison Wesley Longman Publishing Co., Inc., 2004.

COMPENDIUM-JS. 2015.2 Available https://github.com/Ulflander/compendium-js

COX, K.; AURUM, A.; JEFFERY, R. A use case description inspection experiment. In: Australian workshop on software requirements, Sydney, Australia, 2003. Proceedings of Australian workshop on software requirements, 2003.

DAMAS, C.; LAMBEAU, B.; LAMSWEERDE, A. V. Scenarios, goals, and state machines: a win-win partnership for model synthesis. In: ACM SIGSOFT international symposium on Foundations of software engineering, 2006. Proceedings of 14th ACM SIGSOFT international symposium on Foundations of software engineering, ACM, New York, NY, USA, 2006, p. 197-207.

DAMM, W.; HAREL, D. "LSCs: Breathing Life into Message Sequence Charts". Formal Methods in System Design, v. 19, n. 1, p. 45-80, 2001.

DENGER, C.; PAECH, B.; FREIMUT, B. Achieving high quality of usecase-based requirements. Informatik-Forschung und Entwicklung, v. 20, n. 1, p. 11-23, 2005. 
DOWNEY, A. B. The Little Book of Semaphores. Green Tea Press, 2005. Available at: http://greenteapress.com/semaphores.

EASTERBROOK, S. "The Difference between verification and validation", 2010. Available at: http://www.easterbrook.ca/steve/2010/11/thedifference-between-verification-and-validation/

ESHUIS, R.; DEHNERT, J. Application and Theory of Petri Nets. LNCS 2679, 2003, p. 295-314.

FEMMER, H.; FERNÁNDEZ, D. M.; JUERGENS, E.; KLOSE, M.; ZIMMER, I.; ZIMMER, J. Rapid requirements checks with requirements smells: two case studies. In: International Workshop on Rapid Continuous Software Engineering, 2014. Proceedings of the 1st International Workshop on Rapid Continuous Software Engineering, ACM, 2014, p. 1019.

GATE. 2015. Available at: https://gate.ac.uk/

GLINZ, M. Improving the quality of requirements with scenarios, In: World Congress for Software Quality(2WCSQ), 2000, Yokohama. Proceedings of the Second World Congress for Software Quality (2WCSQ), 2000, p. 5560.

GNESI, S.; FABBRINI, F.; FUSANI, M.; TRENTANNI, G. An automatic tool for the analysis of natural language requirements. CSSE Journal, v. 20, n. 1, p. 53-62, 2005.

GRAMMARING. 2015. Available at: http://www.grammaring.com/stateverbs-and-action-verbs

GUTIÉRREZ, J. J.; CLÉMENTINE, N.; ESCALONA, M. J.; MEJÍAS, M.; RAMOS, I. M. Visualization of Use Cases through Automatically Generated Activity Diagrams. In: CZARNECKI, K.; OBER, I.; BRUEL, J. M.; UHL, A.; VÖLTER, M. (eds.) MODELS, 2008, Heidelberg: LNCS, Springer, v. 5301, p. 83-96, 2008.

HAREL, D. StateCharts: a visual formalism for complex systems. SciComput Program, v. 8, n. 3, p. 231-274, 1987.

HEITMEYER, C. Formal methods for specifying, validating, and verifying requirements. J Univ Comput Sci, v. 13, n. 5, 2007, p. 607-618.

HSIA, P.; SAMUEL, J.; GAO, J.; KUNG, D.; TOYOSHIMA, Y.; CHEN, C. Formal Approach to Scenario Analysis. IEEE Software, p. 33-41, 1994.

KELLER, R. Formal Verification of Parallel Programs. Communications of the ACM, v. 19, n. 7, p. 561-572, 1976.

KEPLER PROJECT. 2009. Available at: https://github.com/keplerproject

KLEIN, D.; MANNING, C. D. Accurate unlexicalized parsing. In: Annual Meeting on Association for Computational Linguistics - ACL'03, 2003. Proceedings of the 41st Annual Meeting on Association for Computational Linguistics, 2003, p. 423-430.

IEEE Computer Society. IEEE Recommended Practice for Software Requirements Specications. Technical report, 1998. 
IERUSALIMSCHY, R. Programming in Lua. Lua.org, 3 edition, 2013.

ISO/IEC 14977. Extended Backus-Naur Form. 2015. Available at: http://standards.iso.org/ittf/PubliclyAvailableStandards/s026153_ISO_IEC_ 14977_1996(E).zip

ISO, IEC, and IEEE. ISO/IEC/IEEE 29148:2011. Technical report, ISO IEEE IEC, 2011.

LEE, W.; CHA, S.; KWON, Y. Integration and analysis of use cases using Modular Petri Nets in requirements engineering. IEEE Trans. on Software Engineering, v. 24, n. 12, p. 1115-1130, 1998.

LEE, J.; PAN, J. I.; KUO, J.Y. Verifying scenarios with time petri-nets. Inf. Softw. Technol., v. 43, n. 13, p. 769-781, 2001.

LEITE, J. C. S. P.; HADAD, G; DOORN, J.; KAPLAN, G. A scenario construction process, Requirements Engineering Journal, Springer-Verlag London Limited, v. 5, n. 1, p. 38-61, 2000.

LEITE, J. C. S. P.; DOORN, J. H.; HADAD, G. D.; KAPLAN, G. N. Scenario inspections. Requirements Engineering, v. 10, n. 1, p. 1-21, 2005.

LEITE, J. C. S. P. Livro Vivo: Engenharia de Requisitos. Available at: http://livrodeengenhariaderequisitos.blogspot.com/, 2007.

LEVENSHTEIN, V. I. "Binary codes capable of correcting deletions, insertions, and reversals". Soviet Physics Doklady, v. 10, n. 8, p. 707-710, 1966.

LIU, S.; SUN, J.; LIU, Y.; ZHANG, Y.; WADHWA, B.; DONG, J. S.; WANG, $X$. Automatic early defects detection in use case documents. In: ACM/IEEE international conference on Automated software engineering, 2014. Proceedings of the 29th ACM/IEEE international conference on Automated software engineering, 2014, p. 785-790.

LUCASSEN, G.; DALPIAZ, F.; BRINKKEMPER, S.; VAN DER WERF, J. M. E. M. Forging High-Quality User Stories: Towards a Discipline for Agile Requirements. In: IEEE International Requirements Engineering Conference, 2015. Proceedings of the IEEE International Requirements Engineering Conference, 2015, p. 126-135.

MAVIN, A.; WILKINSON, P.; HARWOOD, A.; NOVAK, M. "Easy approach to requirements syntax (EARS)". In: IEEE International Requirements Engineering Conference (RE'09), 2009. Proceedings of 17th IEEE International Requirements Engineering Conference (RE'09), 2009, p. 317-322.

MURATA, T. Petri nets: Properties, analysis and applications. Proceedings of the IEEE, v. 77, n. 4, p. 541-580, 1989.

NLTK. 2015. Available at: http://text-processing.com/demo/tag/

NOUNANDVERB. Words that are also nouns. 2015. Available at: http://www.enchantedlearning.com/wordlist/nounandverb.shtml

OLSON, D. Advanced data mining techniques. Springer Verlag, 2008. 
OPENNLP. 2015. Available at: http://opennlp.apache.org/

PHALP, K. T.; VINCENT, J.; COX, K. Assessing the quality of use case descriptions. Software Quality Journal, v. 15, n. 1, p. 69-97, 2007.

PIPE2. Platform Independent Petri net Editor 2. 2015. Available at http://pipe2.sourceforge.net.

$\mathrm{POHL}, \mathrm{K}$. The three dimensions of requirements engineering: a framework and its applications. Information Systems Journal, v. 19, n. 3, p. 243-258, 1994.

POHL, K.; RUPP, C. Requirements Engineering Fundamentals, 1st ed. Rocky Nook, 2011.

SARMIENTO, E.; BORGES, M. R. S.; CAMPOS, M. L. M. Applying an event-based approach for detecting requirements interaction, In: International Conference on Enterprise Information Systems (ICEIS 2009), 2009.

SARMIENTO, E.; ALMENTERO, E.; LEITE, J. C. S. P. C\&L: Generating Model Based Test Cases From Natural Language Requirements Descriptions In: IEEE International Workshop on Requirements Engineering and Testing - RET'2014, Sweden, 2014.

SARMIENTO, E.; LEITE, J. C. S. P.; RODRIGUEZ, N.; VON STAA, A. An Automated Approach of Test Case Generation for Concurrent Systems from Requirements Descriptions. In: XVI International Conference on Enterprise Information Systems (ICEIS 2014), Portugal, 2014.

SARMIENTO, E.; ALMENTERO, E.; LEITE, J. C. S. P. Analysis of scenarios with Petri-Net models, In: Brazilian Symposium on Software Engineering - SBES, 2015.

SARMIENTO, E.; ALMENTERO, E; LEITE, J. C. S. P.; SOTOMAYOR, G. Mapping Textual Scenario to Analyzable Petri-Nets. In: XVII International Conference on Enterprise Information Systems (ICEIS 2015), Spain, 2015.

SARMIENTO, E.; ALMENTERO, E.; LEITE, J. C. S. P. Using Correctness, Consistency and Completeness Patterns for Automated Scenarios Analysis. In: 5th IEEE Workshop of Requirements Engineering Patterns RePa, Canada, 2015.

SINHA, A.; SUTTON, S. M.; PARADKAR, A. Text2Test: Automated inspection of natural language use cases. In: International Conference on Software Testing, Verification and Validation (ICST), 2010. Proceedings of Third International Conference on Software Testing, Verification and Validation, 2010, p. 155-164.

SINNIG, D.; CHALIN, P.; KHENDEK, F. LTS semantics for use case models. In: ACM symposium on Applied Computing, 2009. Proceedings of ACM symposium on Applied Computing, 2009, p. 365-370.

SOMÉ, S. S. Formalization of textual use cases based on petri nets. International Journal of Software Engineering and Knowledge Engineering, v. 20, n. 05, p. 695-737, 2010. 
SOMMERVILLE, I. Software Engineering. 9 ed. Boston: Addison-Wesley, 2010.

STANFORD. 2015. Available at: http://nlp.stanford.edu

RAGO, A.; MARCOS, C.; DIAZ-PACE, J.A. Identifying duplicate functionality in textual use cases by aligning semantic actions. Software \& Systems Modeling, p. 1-25, 2014.

REISIG, W. Petri Nets: An Introduction. Heidelberg: Springer-Verlag, 1985.

ROSCOE, A. W. The Theory and Practice of Concurrency. Prentice Hall, 1998.

ROSS, D. T. Structured Analysis (SA): A Language for Communicating Ideas. IEEE Transactions on Software Engineering, v. 3, n. 1, p. 16-34, 1977.

TJONG, S. F. Avoiding ambiguity in requirements specifications, 2008. PhD thesis, University of Nottingham Malaysia Campus, Faculty of Engineering \& Computer Science, Malaysia.

TREEBANK. 2015. Available at: http://www.cis.upenn.edu/ treebank/

UCDB, 2015. Available at: http://www.se.cs.put.poznan.pl/knowledgebase/software-projects-database/use-cases-database-ucdb

UML. Object Management Group, 2015. Available at: http://www.omg.org/spec/UML/

USINGENGLISH. 2015.2 Available http://www.usingenglish.com/glossary/copula-verb.html

VAN LAMSWEERDE, A.; WILLEMET, L. Inferring declarative requirements specifications from operational scenarios. IEEE Transactions on Software Engineering, v. 24, n. 12, p. 1089-1114, 1998.

VAN LAMSWEERDE, A. Goal-oriented requirements engineering: a guided tour. In: Symposium on Requirements Engineering, 2001, Toronto. Proceedings of fifth IEEE International Symposium on Requirements Engineering, 2001, p. 249-262.

WILSON, W. M.; ROSENBERG, L. H.; HYATT, L. E. Automated Analysis of Requirement Specifications. In: International Conference on Software Engineering (ICSE-97), 1997. Proceedings of Nineteenth International Conference on Software Engineering, Boston, 1997.

WORDNET. 2015. Available at: https://wordnet.princeton.edu/

ZHAO, J.; DUAN, Z. Verification of use case with petri nets in requirement analysis. Computational Science and Its Applications-ICCSA, 2009, p. 2942.

ZOWGHI, D.; GERVASI, V. "On the Interplay Between Consistency, Completeness, and Correctness in Requirements Evolution". Information and Software Technology, v. 45, p. 993-1009, 2003. 


\section{Appendix A1 Referential Specification Used as Baseline}

The following scenario descriptions detail the behavior perceived in four systems used in the literature as baseline or referential specification to evaluate the accuracy of defect detection approaches in use cases or scenarios: Online Broker System (Somé, 2010), ATM use cases (Cox et al., 2003), Dlibra CRM (Ciemniewska and Jurkiewicz, 2007) and Mobile News (Ciemniewska and Jurkiewicz, 2007).

The highlighted words or phrases within internal scenario elements (Title, Goal, Context, Resource, Actor, Episodes, Exception), are defect indicators manually detected by Requirements Engineers from the documents, which act as the baseline for the evaluation of our automated analysis approach.

When a defect is detected within scenario element, it is detailed in a new line after the scenario element using the following format: (<Property $>-<$ Type Defect $><$ Detail $>$ ), where "Property" is the quality negatively impacted by the defect, "Type Defect" is the classification of the defect, and "Detail" gives a description of the defect for fixing.

Based on Olson (2008), a defect or error can be classified as following:

- True Positive (TP): A defect is identified by the experts and is detected by the approach (Defect occurs).

- True Negative (TN): A defect is not identified by the experts and is not detected by the approach (Defect does not occur).

- False Positive (FP): A defect is not identified by the experts and is detected by the approach (Defect does not occur).

- False Negative (FN): A defect is identified by the experts and is not detected by the approach (Defect occurs). 


\section{A1.1}

\section{The Online Broker System}

Table 38 shows the quantitative analysis in scenarios (Pre-conditions, Postconditions, Episodes, Exceptions) from Online Broker System, and how they are mapped into Petri-Net elements (Input places, Output places, Transitions).

Table 38 - Quantitative Analysis of Online Broke System

\begin{tabular}{|c|c|c|c|c|c|c|c|c|c|}
\hline $\begin{array}{l}\text { ID } \\
\text { Scenario }\end{array}$ & Scenario & $\begin{array}{l}\text { Num. Pre- } \\
\text { conditions/Conditions/ } \\
\text { Causes/Constraints }\end{array}$ & $\begin{array}{l}\text { Num. } \\
\text { Post- } \\
\text { conditions }\end{array}$ & $\begin{array}{l}\text { Num. } \\
\text { Episodes }\end{array}$ & $\begin{array}{l}\text { Num. } \\
\text { Exceptions }\end{array}$ & $\begin{array}{l}\text { Num. } \\
\text { Input } \\
\text { Places - } \\
\text { Petri-Net }\end{array}$ & $\begin{array}{l}\text { Num. } \\
\text { Transitions } \\
\text { - Petri-Net }\end{array}$ & $\begin{array}{l}\text { Num. } \\
\text { Output } \\
\text { Places - } \\
\text { Petri-Net }\end{array}$ & $\begin{array}{l}\text { Num. } \\
\text { Dummy } \\
\text { Places } \\
\text { Petri-Net }\end{array}$ \\
\hline 1 & $\begin{array}{l}\text { Handle } \\
\text { Payment }\end{array}$ & 1 & 0 & 4 & 1 & 1 & 5 & 0 & 7 \\
\hline 2 & $\begin{array}{l}\text { International } \\
\text { Supplier bid for } \\
\text { order }\end{array}$ & 3 & 1 & 3 & 2 & 3 & 5 & 1 & 7 \\
\hline 3 & $\begin{array}{l}\text { Local Supplier } \\
\text { bid for order }\end{array}$ & 2 & 1 & 4 & 1 & 2 & 5 & 1 & 7 \\
\hline 4 & Process Bids & 2 & 0 & 4 & 0 & 2 & 4 & 0 & 6 \\
\hline 5 & $\begin{array}{l}\text { Register } \\
\text { Customer }\end{array}$ & 1 & 0 & 5 & 1 & 1 & 6 & 0 & 8 \\
\hline 6 & Submit Order & 6 & 0 & 12 & 4 & 6 & 16 & 0 & 18 \\
\hline & Total & 15 & 2 & 32 & 9 & 15 & 41 & 2 & 53 \\
\hline
\end{tabular}

Table 39 shows the Unambiguity analysis (qualitative) in scenarios (Title, Goal, Episodes, Exceptions) from Online Broker System. Let $\boldsymbol{T P}$ be the number of defects detected correctly by out analysis approach; $\boldsymbol{F P}$ be the number of defects detected incorrectly (defect does not occur); $\boldsymbol{F} N$ be the number of defects that are not detected (defect occurs).

Table 39 - Unambiguity Analysis of Online Broke System

\begin{tabular}{|c|c|c|c|c|c|c|c|c|c|c|c|c|c|c|c|c|c|c|c|c|c|}
\hline \multirow[t]{2}{*}{ Scenario } & \multicolumn{3}{|c|}{ Vague } & \multicolumn{3}{|c|}{ Subjective } & \multicolumn{3}{|c|}{ Optional } & \multicolumn{3}{|c|}{ Weak } & \multicolumn{3}{|c|}{ Multiple } & \multicolumn{3}{|c|}{ Implicit } & \multicolumn{3}{|c|}{ Quantifiable } \\
\hline & TP & FP & FN & TP & FP & FN & TP & FP & FN & TP & FP & FN & TP & FP & FN & TP & FP & FN & TP & FP & FN \\
\hline 1 & & & & & & & & & & & & & & & & 1 & & & & & \\
\hline 2 & & & & & & & & & & & & & 1 & & & & & & & & \\
\hline 3 & & & & & & & & & & & & & 2 & & & & & & & & \\
\hline 4 & & & & & & & & & & & & & & & & & & & & & \\
\hline 5 & & & & & & & & & & & & & & 1 & & & & & & & \\
\hline 6 & 1 & & & & & & & & & & & & & & & 1 & & & & & \\
\hline Total & 1 & 0 & $\mathbf{0}$ & 0 & $\mathbf{0}$ & 0 & 0 & $\mathbf{0}$ & 0 & 0 & $\mathbf{0}$ & 0 & 3 & 1 & $\mathbf{0}$ & 2 & 0 & $\mathbf{0}$ & 0 & 0 & 0 \\
\hline
\end{tabular}

Table 40 shows the Completeness analysis (qualitative) in scenarios (Title, Goal, Episodes, Exceptions) from Online Broker System.

Table 40 - Completeness Analysis of Online Broke System

\begin{tabular}{|c|c|c|c|c|c|c|c|c|c|c|c|c|c|c|c|c|c|c|c|c|c|c|c|c|}
\hline \multirow[t]{2}{*}{$\begin{array}{c}\text { ID } \\
\text { Scenario }\end{array}$} & \multicolumn{3}{|c|}{ Atomicity } & \multicolumn{3}{|c|}{ Simplicity } & \multicolumn{3}{|c|}{ Uniformity } & \multicolumn{3}{|c|}{ Usefulness } & \multicolumn{3}{|c|}{$\begin{array}{c}\text { Conceptually } \\
\text { Soundness }\end{array}$} & \multicolumn{3}{|c|}{ Integrity } & \multicolumn{3}{|c|}{ Coherency } & \multicolumn{3}{|c|}{ Uniqueness } \\
\hline & TP & FP & FN & TP & $\mathbf{F P}$ & FN & TP & FP & FN & TI & FP & FN & TP & FP & $\overline{F N}$ & TP & FP & FN & TP & $\mathbf{F P}$ & FN & TP & FP & FN \\
\hline 1 & & & & 1 & & & & & & & & & & & & & & & & & & & & \\
\hline 2 & & & & 1 & & & & & & 1 & & & 1 & & & & & & & & & 2 & & \\
\hline 3 & & & & 2 & & & & & & 1 & & & 1 & & & & & & & & & 2 & & \\
\hline 4 & & & & 1 & & & & & & 1 & & & & & & & & & & & & & & \\
\hline 5 & & & & 1 & & & & & & & & & & & & & & & & & & & & \\
\hline 6 & & & & 1 & & & & & & 3 & & & 1 & & & & & & & & & & & \\
\hline Total & 0 & 0 & 0 & 7 & 0 & 0 & 0 & 0 & 0 & 6 & 0 & 0 & 3 & 0 & 0 & 0 & 0 & 0 & 0 & 0 & 0 & 4 & 0 & 0 \\
\hline
\end{tabular}


Table 41 shows the Consistency analysis (qualitative) in scenarios and related scenarios from Online Broker System.

\section{Table 41 - Consistency Analysis of Online Broke System}

\begin{tabular}{|c|c|c|r|c|c|c|c|c|c|c|c|c|}
\hline \multirow{2}{*}{ ID Scenario } & \multicolumn{3}{|c|}{ Non-interferential } & \multicolumn{3}{|c|}{ Boundedness } & \multicolumn{2}{|c|}{ Reversibility } & \multicolumn{3}{|c|}{ Liveness } \\
\hline & TP & FP & FN & TP & FP & FN & TP & FP & FN & TP & FP & FN \\
\hline $\mathbf{1}$ & & & & & & & & & & & & \\
\hline $\mathbf{2}$ & 1 & & & & & & & & & & & \\
\hline $\mathbf{3}$ & 1 & & & & & & & & & & & \\
\hline $\mathbf{4}$ & & & & & & & & & & & & \\
\hline $\mathbf{5}$ & & & & & & & & & & & & \\
\hline $\mathbf{6}$ & & & & & & & & & & & & \\
\hline Total & $\mathbf{2}$ & $\mathbf{0}$ & $\mathbf{0}$ & $\mathbf{0}$ & $\mathbf{0}$ & $\mathbf{0}$ & $\mathbf{0}$ & $\mathbf{0}$ & $\mathbf{0}$ & $\mathbf{0}$ & $\mathbf{0}$ & $\mathbf{0}$ \\
\hline
\end{tabular}

TITLE: Handle Payment

GOAL: Handle Payment

CONTEXT: Handle payment for a Bid

PRE-CONDITION:

POST-CONDITION:

ACTOR: Customer, Broker System, Payment System

RESOURCES: Credit card information

\section{EPISODES}

1. The Broker System asks the Customer for Credit Card information

2. The Customer provides her Credit Card information

(Implicit - TP: ambiguous indicator)

3. The Broker System asks a Payment System to process the Customer's Payment.

(Simplicity - TP: Contains more than one Action-Verb)

4. The Broker System displays an acknowledgement message to the Customer

EXCEPTIONS

1.1 IF The Customer Payment is denied THEN The Broker System displays a payment denied page

TITLE: International Supplier bid for order

(Soundness - TP: Title does not describe the Goal)

(Non-interferential - TP: Simultaneous enabled with Local Supplier)

(Uniqueness - TP: Local Supplier bid for order and International Supplier bid for order are potentially duplicated)

GOAL: Submit a bid

CONTEXT: Create a Bid for an Order

PRE-CONDITION: An Order has been broadcasted

(Uniqueness - TP: Context Pre-condition coincidence with Related scenario's Local Supplier)

POST-CONDITION: International Supplier has bidden

ACTOR: International Supplier, Broker System

RESOURCES: Order, Bid

\section{EPISODES}

1. International Supplier receives the Order and examines it

(Multiplicity - TP: ambiguous indicator)

(Simplicity - TP: Contains more than one Action-Verb)

2. International Supplier submits a Bid for the Order

3. The Broker System sends the Bid to the Customer

(Usefulness - TP: Actor/Resource mentioned in episode is not included in the Actor/Resource element)

\section{EXCEPTIONS}

1.1 IF The Order includes items restricted for exportation THEN International Supplier passes on the Order

1.2 IF International Supplier can not satisfy the Order THEN International Supplier passes on the Order 
(Soundness - TP: Title does not describe the Goal)

(Non-interferential - TP: Simultaneous enabled with International Supplier)

(Uniqueness - TP: Local Supplier bid for order and International Supplier bid for order are potentially duplicated)

GOAL: Submit a bid

CONTEXT: Create a Bid for an Order

PRE-CONDITION: An Order has been broadcasted

(Uniqueness - TP: Context Pre-condition coincidence with Related scenario's International

Supplier)

POST-CONDITION: Local Supplier has bidden

ACTOR: Local Supplier, Broker System, Customer

RESOURCES: Order, Bid

\section{EPISODES}

1. Local Supplier receives the Order and examines it

(Multiplicity - TP: ambiguous indicator)

(Simplicity - TP: Contains more than one Action-Verb)

2. Local Supplier determines the applicable taxes to the order and creates a bid.

(Multiplicity - TP: ambiguous indicator)

(Simplicity - TP: Contains more than one Action-Verb)

3. Local Supplier submits a Bid for the Order

4. The Broker System sends the Bid to the Customer.

(Usefulness - TP: Actor/Resource mentioned in episode is not included in the Actor/Resource element)

EXCEPTIONS

1.1 IF Local Supplier can not satisfy the Order THEN Local Supplier passes on the Order

\section{TITLE: Process Bids}

GOAL: Process a bid

CONTEXT: Process a Bid for an Order

PRE-CONDITION: Local Supplier has bidden OR International Supplier has bidden

ACTOR: Customer, Broker System

(Usefulness - TP: never participates in episodes)

RESOURCES: Order, Bid

\section{EPISODES}

1. Customer examines the bid

2. Customer signals the system to proceed with bid

3. HANDLE PAYMENT

4. System put an order with the selected bidder

(Simplicity - TP: Missing Action-Verb in Present Tense form)

TITLE: Register Customer

GOAL: Register Customer

CONTEXT: login page loaded

PRE-CONDITION:

POST-CONDITION:

ACTOR: Customer, Broker System

RESOURCES: registration operation, name, date of birth, address, login information

\section{EPISODES}

1. Customer selects registration operation

2. Broker System asks for Customer name, date of birth and address

(Multiplicity - FP: ambiguous indicator)

3. Customer enters registration information

4. Broker System validates Customer information

5. Broker System generate login information for Customer

(Simplicity - TP: Missing Action-Verb in Present Tense form)

EXCEPTIONS

4.1. IF Customer registration information is not valid THEN Broker System displays registration failure page

TITLE: Submit Order 
(Soundness: Title does not describe the Goal)

GOAL: Allow customers to find the best supplier for a given order.

CONTEXT:

PRE-CONDITION: The Broker System is online AND the Broker System welcome page is being displayed

ACTOR: Customer, Broker System

RESOURCES: Login page, Login information, Order

EPISODES

(Usefulness - TP: Too long scenario - Num. episodes > 10)

1. The Customer loads the login page

2. The Broker System asks for the Customer's login information

3. The Customer enters her login information

(Implicit - TP: ambiguous indicator)

4. The Broker System checks the provided login information

(Vagueness - TP: ambiguous indicator)

5. The Broker System displays an order page

6. The Customer creates a new Order

7. DO the Customer adds an item to the Order WHILE the Customer has more items to add to the order

8. The Customer submits the Order

9. The Broker System broadcast the Order to the Suppliers

(Simplicity - TP: Missing Action-Verb in Present Tense form)

10. \# LOCAL SUPPLIER BID FOR ORDER.

(Usefulness - TP: Actor/Resource mentioned in episode is not included in the Actor/Resource element)

11. INTERNATIONAL SUPPLIER BID FOR ORDER \#

(Usefulness - TP: Actor/Resource mentioned in episode is not included in the Actor/Resource element)

12. PROCESS BIDS

\section{EXCEPTIONS}

1.1 IF Customer is not registered THEN REGISTER CUSTOMER

2.1 IF after 60 seconds THEN The Broker System displays a login timeout page.

4.1 IF the Customer login information is not accurate THEN The Broker System displays an alert message

8.1 IF the order is empty THEN The Broker System displays an error message

\section{A1.2}

\section{The ATM System}

Table 42 shows the quantitative analysis in scenarios (Pre-conditions, Postconditions, Episodes, Exceptions) from ATM System, and how they are mapped in Petri-Net elements (Input places, Output places, Transitions).

Table 42 - Quantitative Analysis of ATM System

\begin{tabular}{|c|c|c|c|c|c|c|c|c|c|}
\hline $\begin{array}{l}\text { ID } \\
\text { Scenario }\end{array}$ & Scenario & $\begin{array}{l}\text { Num. Pre- } \\
\text { conditions/Conditions/ } \\
\text { Causes/Constraints }\end{array}$ & $\begin{array}{l}\text { Num. } \\
\text { Post- } \\
\text { conditions }\end{array}$ & $\begin{array}{l}\text { Num. } \\
\text { Episodes }\end{array}$ & $\begin{array}{l}\text { Num. } \\
\text { Exceptions }\end{array}$ & $\begin{array}{l}\text { Num. } \\
\text { Input } \\
\text { Places } \\
\text { - Petri- } \\
\text { Net }\end{array}$ & $\begin{array}{l}\text { Num. } \\
\text { Transitions } \\
\text { - Petri-Net }\end{array}$ & $\begin{array}{l}\text { Num. } \\
\text { Output } \\
\text { Places - } \\
\text { Petri- } \\
\text { Net }\end{array}$ & $\begin{array}{l}\text { Num. } \\
\text { Dummy } \\
\text { Places - } \\
\text { Petri- } \\
\text { Net }\end{array}$ \\
\hline 1 & ACCESS ATM & 1 & 3 & 6 & 1 & 1 & 7 & 3 & 9 \\
\hline 2 & CHANGE PIN & 2 & 2 & 7 & 1 & 2 & 8 & 2 & 10 \\
\hline 3 & CHECK BALANCE & 2 & 2 & 5 & 1 & 2 & 6 & 2 & 8 \\
\hline 4 & MAKE DEPOSIT & 2 & 1 & 4 & 1 & 2 & 5 & 1 & 7 \\
\hline & $\begin{array}{rr}\text { Total } \\
\end{array}$ & 8 & 9 & 33 & 5 & 8 & 38 & 9 & 48 \\
\hline
\end{tabular}

Table 43 shows the Unambiguity analysis (qualitative) in scenarios (Title,

Goal, Episodes, Exceptions) from ATM System. Let $\boldsymbol{T P}$ be the number of defects 
detected correctly by out analysis approach; $\boldsymbol{F P}$ be the number of defects detected incorrectly (defect does not occur); $\boldsymbol{F N}$ be the number of defects that are not detected (defect occurs).

Table 43 - Unambiguity Analysis of ATM System

\begin{tabular}{|c|c|c|c|c|c|c|c|c|c|c|c|c|c|c|c|c|c|c|c|c|c|}
\hline \multirow[t]{2}{*}{ Scenario } & \multicolumn{3}{|c|}{ Vague } & \multicolumn{3}{|c|}{ Subjective } & \multicolumn{3}{|c|}{ Optional } & \multicolumn{3}{|c|}{ Weak } & \multicolumn{3}{|c|}{ Multiple } & \multicolumn{3}{|c|}{ Implicit } & \multicolumn{3}{|c|}{ Quantifiable } \\
\hline & TP & FP & FN & TP & FP & FN & TP & FP & FN & TP & FP & FN & TP & FP & FN & TP & FP & FN & TP & FP & FN \\
\hline 1 & & & & & & & & & & & & & 1 & & & 1 & & & & & \\
\hline 2 & & & & & & & & & & & & & & & & 3 & & & & & \\
\hline 3 & & & & & & & & & & & & & & & & 2 & & & & & \\
\hline 4 & & & & & & & & & & & & & & & & & & & & & \\
\hline 5 & & & & & & & & & & & & & & & & 2 & & & & & \\
\hline Total & $\mathbf{0}$ & $\mathbf{0}$ & $\mathbf{0}$ & $\mathbf{0}$ & $\mathbf{0}$ & $\mathbf{0}$ & 0 & $\mathbf{0}$ & $\mathbf{0}$ & $\mathbf{0}$ & $\mathbf{0}$ & 0 & 1 & $\mathbf{0}$ & $\mathbf{0}$ & 8 & $\mathbf{0}$ & $\mathbf{0}$ & $\mathbf{0}$ & $\mathbf{0}$ & 0 \\
\hline
\end{tabular}

Table 44 shows the Completeness analysis (qualitative) in scenarios (Title, Goal, Episodes, Exceptions) from ATM System.

Table 44 - Completeness Analysis of ATM System

\begin{tabular}{|c|c|c|c|c|c|c|c|c|c|c|c|c|c|c|c|c|c|c|c|c|c|c|c|c|}
\hline \multirow[t]{2}{*}{$\begin{array}{l}\text { ID } \\
\text { Scenario }\end{array}$} & \multicolumn{3}{|c|}{ Atomicity } & \multicolumn{3}{|c|}{ Simplicity } & \multicolumn{3}{|c|}{ Uniformity } & \multicolumn{3}{|c|}{ Usefulness } & \multicolumn{3}{|c|}{$\begin{array}{l}\text { Conceptually } \\
\text { Soundness }\end{array}$} & \multicolumn{3}{|c|}{ Integrity } & \multicolumn{3}{|c|}{ Coherency } & \multicolumn{3}{|c|}{ Uniqueness } \\
\hline & TP & FP & FN & TP & FP & FN & TP & FP & FN & TP & FP & FN & TP & FP & FN & TP & FP & FN & TP & FP & FN & TP & FP & FN \\
\hline 1 & & & & 1 & & & 1 & & & 1 & & & & & & & & & & & & & & \\
\hline 2 & & & & 3 & 1 & & & & & & & & & & & & & & & & & & & \\
\hline 3 & & & & & & & & & & & & & & 1 & & & & & & & & & & \\
\hline 4 & & & & 1 & & & & & & & & & & 1 & & & & & & & & & & \\
\hline 5 & & & & 2 & & & 1 & & & 1 & & & & 1 & & & & & & & & & & \\
\hline Total & 0 & 0 & 0 & 7 & 1 & 0 & 2 & 0 & 0 & 2 & 0 & 0 & 0 & 3 & 0 & 0 & 0 & 0 & 0 & 0 & 0 & 0 & 0 & 0 \\
\hline
\end{tabular}

Table 45 shows the Consistency analysis (qualitative) in scenarios and related scenarios from ATM System.

Table 45 - Consistency Analysis of ATM System

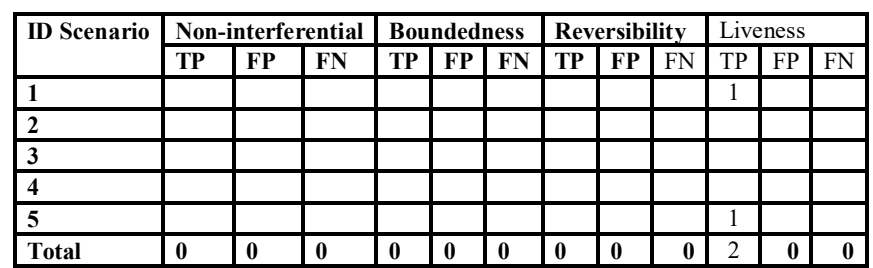

TITLE: ACCESS ATM

GOAL: User access the ATM.

Context: User wants to use the ATM.

Pre-condition: ATM in ready state for new User

Post-condition: User access granted AND PIN AND card validated.

ACTOR: User

RESOURCE: ATM, card, PIN, account EPISODES:

1. User inserts card into ATM.

2. ATM asks for a PIN.

3. User types in the numbers of his PIN and presses the Enter button

(Multiplicity - TP: ambiguous indicator)

(Implicit - TP: ambiguous indicator)

(Simplicity - TP: Contains more than one Action-Verb)

4. ATM asks for account type.

5. Customer selects account.

(Usefulness - TP: Actor/Resource mentioned in episode is not included in the Actor/Resource element)

6. ATM displays User options.

EXCEPTION: 
4.1. ATM rejects unidentifiable card.

(Uniformity - TP: Incomplete Cause)

(Liveness - TP: Never enabled transition)

\section{TITLE: CHANGE PIN}

GOAL: User wants to change their PIN.

(Implicit - TP: ambiguous indicator)

Context:

Pre-condition: User already logged onto the ATM

Post-condition: New PIN read to card and Bank account

ACTOR: User

RESOURCE: ATM, PIN

\section{EPISODES:}

1. User selects Change PIN.

2. ATM prompts her to enter new PIN.

(Implicit - TP: ambiguous indicator)

(Simplicity - TP: Contains more than one Action-Verb)

3. It enters new PIN.

(Implicit - TP: ambiguous indicator)

(Simplicity - TP: Missing Subject)

4. ATM prompts User to re-enter new PIN.

5. User re-enters new PIN.

(Simplicity - FP: Contains more than one Subject, Missing Action-Verb)

6. ATM displays New PIN Successful message.

7. ATM displays list of options.

\section{EXCEPTION:}

4.1. IF ATM refuses new PIN THEN User asked to re-enter new PIN.

(Simplicity - TP: Missing Action-Verb in Present Tense form)

\section{TITLE: CHECK BALANCE}

(Soundness - FP: Title does not describe the Goal)

GOAL: The User wants to check their account balance before withdrawing money.

(Implicit - TP: ambiguous indicator)

Context:

Pre-condition: User already logged onto the ATM.

Post-condition: Balance no longer displayed AND ATM ready for a transaction.

ACTOR: User, Bank

RESOURCE: ATM, account

\section{EPISODES:}

1. User selects balance of account.

2. User selects On Screen option.

3. ATM displays current balance on screen.

4. Bank retrieves User's current balance from their account.

(Implicit - TP: ambiguous indicator)

5. ATM prompts for new option.

\section{EXCEPTION:}

2.1. IF User selects On Paper option THEN ATM prints balance on receipt.

\section{TITLE: MAKE DEPOSIT}

(Soundness - FP: Title does not describe the Goal)

GOAL: The User wants to deposit cash into the ATM

Context:

Pre-condition: The User has logged onto the ATM .

Post-condition: ATM ready for a new transaction.

ACTOR: User

RESOURCE: ATM, envelope, deposit

EPISODES:

1. User selects Deposit.

2. Selects envelope

(Simplicity - TP: Missing Subject) 
3. ATM accepts deposit

4. User takes deposit receipt.

EXCEPTION:

3.1. IF ATM rejects deposit envelope THEN ATM signals User of rejection.

\section{TITLE: WITHDRAW CASH}

(Soundness - FP: Title does not describe the Goal)

GOAL: User wants to withdraw money.

Context: User wants to use the ATM.

Pre-condition: User has already logged onto the ATM.

Post-condition: ATM ready for next User.

ACTOR: User, Bank

RESOURCE: ATM, account, card

EPISODES:

(Usefulness - TP: Too long scenario - Num. episodes $>10$ )

1. User selects Withdraw Cash.

2. ATM prompts for amount.

3. User enters amount.

4. ATM verifies with the Bank that the User has enough money in account.

(Implicit - TP: ambiguous indicator)

4.1 If insufficient funds in her account,

(Implicit - TP: ambiguous indicator)

(Simplicity - TP: Nested Episode Sentence must be treated by a scenario)

4.2 ATM returns card to User.

4.3 User takes card.

5. ATM releases cash.

6. User takes cash.

7. ATM releases card.

8. User takes card.

(Simplicity - TP: Episode Sentence coincidence with episode "4.3")

\section{EXCEPTION:}

7.1. ATM eats card.

(Uniformity - TP: Incomplete Cause)

(Liveness - TP: Never enabled transition) 


\section{A1.3 \\ DLibra CRM}

Table 46 shows the quantitative analysis in scenarios (Pre-conditions, Postconditions, Episodes, Exceptions) from Online Broker System, and how they are mapped in Petri-Net elements (Input places, Output places, Transitions).

Table 46 - Quantitative Analysis of Online Broke System

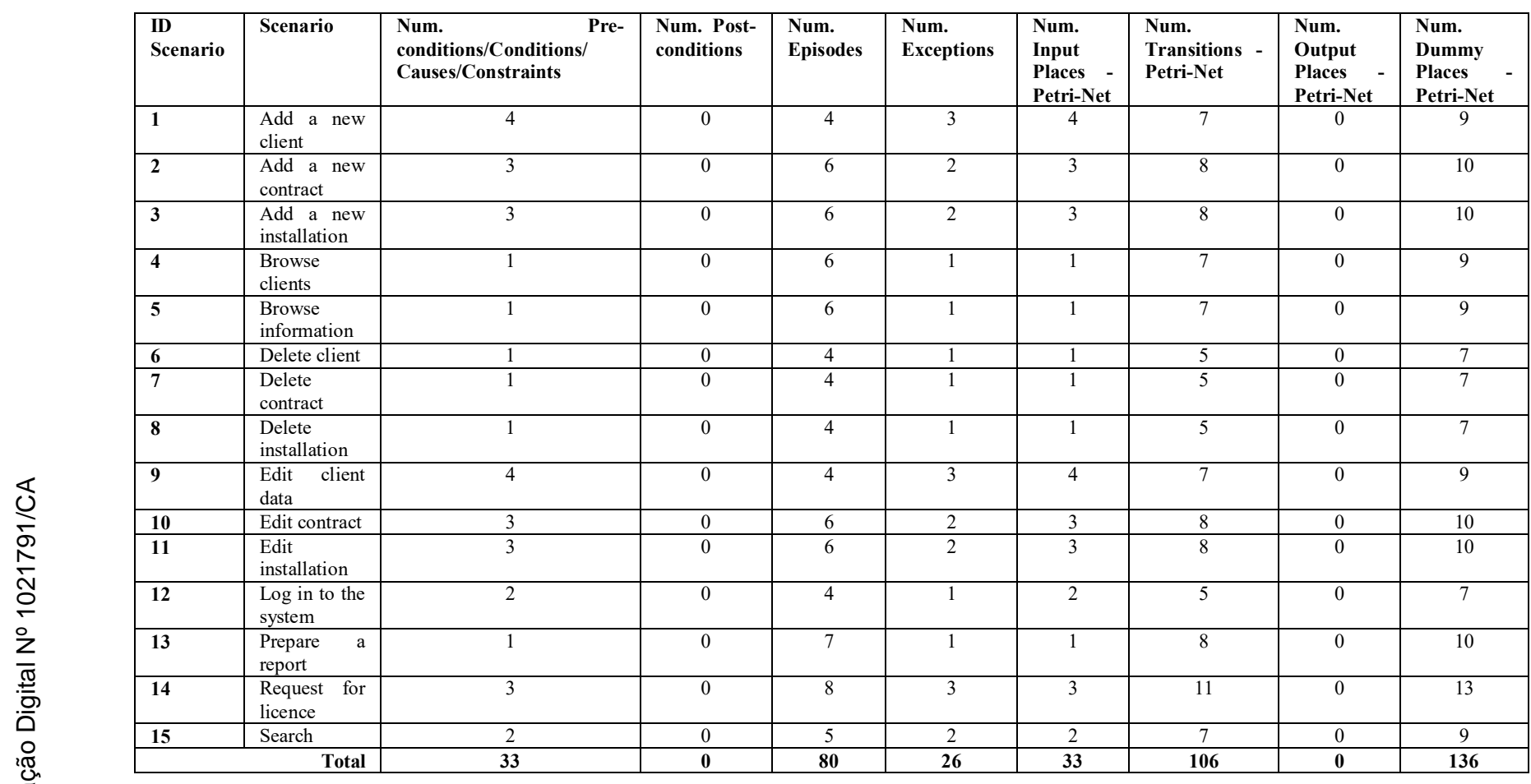

Table 47 shows the Unambiguity analysis (qualitative) in scenarios (Title, Goal, Episodes, Exceptions) from Dlibra System. Let $\boldsymbol{T P}$ be the number of defects detected correctly by out analysis approach; $\boldsymbol{F P}$ be the number of defects detected incorrectly (defect does not occur); $\boldsymbol{F N}$ be the number of defects that are not detected (defect occurs).

Table 47 - Unambiguity Analysis of Dlibra System

\begin{tabular}{|c|c|c|c|c|c|c|c|c|c|c|c|c|c|c|c|c|c|c|c|c|c|}
\hline \multirow[t]{2}{*}{ ID Scenario } & \multicolumn{3}{|c|}{ Vague } & \multicolumn{3}{|c|}{ Subjective } & \multicolumn{3}{|c|}{ Optional } & \multicolumn{3}{|c|}{ Weak } & \multicolumn{3}{|c|}{ Multiple } & \multicolumn{3}{|c|}{ Implicit } & \multicolumn{3}{|c|}{ Quantifiable } \\
\hline & TP & FP & FN & TP & FP & FN & TP & FP & FN & TP & FP & FN & TP & FP & FN & TP & FP & FN & TP & FP & FN \\
\hline 1 & & & & & & & & & & & & & 1 & & & 1 & & & 1 & & \\
\hline 2 & & & & & & & & & & 1 & & & 1 & & & 3 & & & 1 & & \\
\hline 3 & & & & & & & & & & 1 & & & 1 & & & 3 & & & & & \\
\hline 4 & & & & & & & & & & 1 & & & 1 & & & & & & & & \\
\hline 5 & & & & & & & & & & 3 & & & 2 & & & & & & & & \\
\hline 6 & & & & & & & & & & & & & 1 & & & & & & & & \\
\hline 7 & & & & & & & & & & & & & 1 & & & & & & & & \\
\hline 8 & & & & & & & & & & & & & 1 & & & & & & & & \\
\hline 9 & & & & & & & & & & & & & 1 & & & 1 & & & & & \\
\hline 10 & & & & & & & & & & & & & & & & 1 & & & & & \\
\hline 11 & & & & & & & & & & & & & & & & 1 & & & & & \\
\hline 12 & & & & & & & & & & & & & & & & & & & 1 & & \\
\hline 13 & & & & & & & & & & & & & 1 & 2 & & & & & & & \\
\hline 14 & 1 & & & & & & & & & & & & 1 & & & 1 & & & 1 & & \\
\hline 15 & & & & & & & & & & & & & & & & 1 & & & & & \\
\hline Total & 1 & $\mathbf{0}$ & $\mathbf{0}$ & $\mathbf{0}$ & $\mathbf{0}$ & $\mathbf{0}$ & $\mathbf{0}$ & $\mathbf{0}$ & $\mathbf{0}$ & 6 & $\mathbf{0}$ & $\mathbf{0}$ & 12 & 2 & $\mathbf{0}$ & 12 & $\mathbf{0}$ & $\mathbf{0}$ & 4 & $\mathbf{0}$ & $\mathbf{0}$ \\
\hline
\end{tabular}


Table 48 shows the Completeness analysis (qualitative) in scenarios (Title, Goal, Episodes, Exceptions) from Dlibra System. Consistency is not shown because we do not have a baseline manually identified by Requirements Engineers.

Table 48 - Completeness Analysis of Dlibra System

\begin{tabular}{|c|c|c|c|c|c|c|c|c|c|c|c|c|c|c|c|c|c|c|c|c|c|c|c|c|}
\hline \multirow[t]{2}{*}{$\begin{array}{l}\text { ID } \\
\text { Scenario }\end{array}$} & \multicolumn{3}{|c|}{ Atomicity } & \multicolumn{3}{|c|}{ Simplicity } & \multicolumn{3}{|c|}{ Uniformity } & \multicolumn{3}{|c|}{ Usefulness } & \multicolumn{3}{|c|}{$\begin{array}{l}\text { Conceptually } \\
\text { Soundness }\end{array}$} & \multicolumn{3}{|c|}{$\begin{array}{l}\text { Integrity } \\
\end{array}$} & \multicolumn{3}{|c|}{ Coherency } & \multicolumn{3}{|c|}{ Uniqueness } \\
\hline & TP & FP & FN & TP & $\mathbf{F P}$ & FN & TP & FP & FN & TP & FP & FN & TP & FP & FN & TP & $\mathbf{F P}$ & FN & TP & FP & FN & TP & FP & FN \\
\hline 1 & & & & 2 & & & & & & & & & & & & & & & & & & & & \\
\hline 2 & & & & 1 & & & & & & & & & & & & & & & & & & & & \\
\hline 3 & & & & 1 & & & & & & & & & & & & & & & & & & & & \\
\hline 4 & & & & 5 & & & & & & & & & & & & & & & & & & & & \\
\hline 5 & & & & 5 & & & & & & & & & & & & & & & & & & & & \\
\hline 6 & & & & 3 & & & & & & & & & & & & & & & & & & & & \\
\hline 7 & & & & 3 & & & & & & & & & & & & & & & & & & & & \\
\hline 8 & & & & 3 & & & & & & & & & & & & & & & & & & & & \\
\hline 9 & & & & 2 & & & & & & & & & & & & & & & & & & & & \\
\hline 10 & & & & 1 & & & & & & & & & & & & & & & & & & & & \\
\hline 11 & & & & 1 & & & & & & & & & & & & & & & & & & & & \\
\hline 12 & & & & & & & & & & & & & & & & & & & & & & & & \\
\hline 13 & & & & 3 & & & & & & & & & & & & & & & & & & & & \\
\hline 14 & & & & 5 & & & & & & & & & & & & & & & & & & & & \\
\hline 15 & 1 & & & & & & & & & & & & & 1 & & & & & & & & & & \\
\hline Total & 1 & 0 & 0 & 35 & 0 & 0 & 0 & 0 & 0 & 0 & 0 & 0 & 0 & 1 & 0 & 0 & 0 & 0 & 0 & 0 & 0 & 0 & 0 & 0 \\
\hline
\end{tabular}

TITLE: Add a new client

GOAL: Add a new client

CONTEXT: user Add a new client

ACTOR: User, System

RESOURCE: database, clients

EPISODES:

1. User select option for adding new clients.

(Simplicity - TP: Missing Action-Verb in Present Tense)

2. User fills all required personal client data forms.

(Quantifiable - TP: ambiguous indicator)

3. System verifies correctness of data.

4. System adds a new client to the database and informs user about it.

(Multiplicity - TP: ambiguous indicator)

(Simplicity - TP: Contains more than one Action-Verb)

EXCEPTION:

3.1. IF Data is incomplete or incorrect THEN System informs user about problems.

3.2. IF Client with same personal data already exists THEN System informs user about that fact.

(Implicit - TP: ambiguous indicator)

4.1. IF Client can't be added THEN System informs user about reason why client can't be added.

TITLE: $\quad$ Add a new contract

GOAL: Add a new contract

CONTEXT: Add a new contract

ACTOR: User, System

RESOURCE: client, contract

EPISODES:

1. User select a client for whom new contract will be added.

(Simplicity - TP: Missing Action-Verb in Present Tense)

(Implicit - TP: ambiguous indicator)

(Weakness - TP: ambiguous indicator)

2. User chooses option for adding new contract.

3. System displays transaction form.

4. User fills all required data.

(Quantifiable - TP: ambiguous indicator)

5. System verifies information.

6. System saves contract and bounds it to the selected client. 
(Multiplicity - TP: ambiguous indicator)

(Implicit - TP: ambiguous indicator)

EXCEPTION:

5.1. IF Data is incomplete or incorrect THEN System informs user about problems.

6.1. IF contract can't be saved THEN System informs user about that fact.

(Implicit - TP: ambiguous indicator)

TITLE: Add a new installation

GOAL: Add a new installation

CONTEXT: Add a new installation

ACTOR: User, System

RESOURCE: client, installation

EPISODES:

1. User select a client for whom new installation will be added.

(Simplicity - TP: Missing Action-Verb in Present Tense)

(Implicit - TP: ambiguous indicator)

(Weakness - TP: ambiguous indicator)

2. User chooses option for adding new installation.

3. System displays installation form.

4. User fills required data.

5. System verifies information.

6. System saves installation and bounds it to the selected client.

(Multiplicity - TP: ambiguous indicator)

(Implicit - TP: ambiguous indicator)

EXCEPTION:

5.1. IF Data is incomplete or incorrect THEN System informs user about problems.

6.1. IF Installation can't be saved THEN System informs user about that fact.

(Implicit - TP: ambiguous indicator)

\section{TITLE: $\quad$ Browse clients}

GOAL: Browse clients

CONTEXT: Browse clients

ACTOR: user, System

RESOURCE: clients, scenario

EPISODES:

1. User select option for browsing clients.

(Simplicity - TP: Missing Action-Verb in Present Tense)

2. System displays the list of clients.

3. User may filter clients with specified criteria.

(Simplicity - TP: Missing Action-Verb in Present Tense) (Weakness: ambiguous indicator)

4. User may sort clients.

(Simplicity - TP: Missing Action-Verb in Present Tense)

(Weakness - TP: ambiguous indicator)

5. User may view details about selected client.

(Simplicity - TP: Missing Action-Verb in Present Tense)

(Weakness - TP: ambiguous indicator)

6. Scenario ends when user logs out or selects different option.

(Multiplicity - TP: ambiguous indicator)

(Simplicity - TP: Contains more than one Action-Verb)

EXCEPTION:

2.1. IF There are no clients to display THEN System displays blank list.

\section{TITLE: Browse information}

GOAL: Browse information

CONTEXT: Browse information

ACTOR: User, System

RESOURCE: licences, keys, contracts, installations, scenario

EPISODES:

1. User select option for browsing data.

(Simplicity - TP: Missing Action-Verb in Present Tense) 
2. System displays the list of licences, keys, contracts and installations (grouping by type).

(Multiplicity - TP: ambiguous indicator)

3. User may filter data with specified criteria.

(Simplicity - TP: Missing Action-Verb in Present Tense)

(Weakness - TP: ambiguous indicator)

4. User may sort data.

(Simplicity - TP: Missing Action-Verb in Present Tense)

(Weakness - TP: ambiguous indicator)

5. User may view details about selected element.

(Simplicity - TP: Missing Action-Verb in Present Tense)

(Weakness - TP: ambiguous indicator)

6. Scenario ends when users logs out or select different option.

(Multiplicity - TP: ambiguous indicator)

(Simplicity - TP: Contains more than one Action-Verb)

EXCEPTION:

2.1. IF There are no data to display THEN System displays blank list.

TITLE: Delete client

GOAL: Delete client

CONTEXT: Delete client

ACTOR: User, System

RESOURCE: client, clients, database

EPISODES:

1. User select option for deleting clients.

(Simplicity - TP: Missing Action-Verb in Present Tense)

2. User delete chosen client.

(Simplicity - TP: Missing Action-Verb in Present Tense)

3 . System verifies possibility to perform deleting.

4. System saves changes to the database and informs user about it.

(Multiplicity - TP: ambiguous indicator)

(Simplicity - TP: Contains more than one Action-Verb)

EXCEPTION:

3.1. IF Client can not be deleted THEN System informs user about the conditions.

\section{TITLE: Delete contract}

GOAL: Delete contract

CONTEXT: Delete contract

ACTOR: User, System

RESOURCE: contract, database

EPISODES:

1. User select option for deleting contract.

(Simplicity - TP: Missing Action-Verb in Present Tense)

2. User delete chosen contract.

(Simplicity - TP: Missing Action-Verb in Present Tense)

3. System verifies possibility to perform deleting.

4. System saves changes to the database and informs user about it.

(Multiplicity - TP: ambiguous indicator)

\section{EXCEPTION:}

(Simplicity - TP: Contains more than one Action-Verb)

3.1. IF Contract can not be deleted THEN System informs user about problems.

\section{TITLE: Delete installation}

GOAL: Delete installation

CONTEXT: Delete installation

ACTOR: User, System

RESOURCE: installation, database

EPISODES:

1. User select option for deleting installation.

(Simplicity - TP: Missing Action-Verb in Present Tense)

2. User delete chosen installation. 
(Simplicity - TP: Missing Action-Verb in Present Tense)

3. System verifies possibility to perform deleting.

4. System saves changes to the database and informs user about it.

(Multiplicity - TP: ambiguous indicator)

\section{EXCEPTION:}

(Simplicity - TP: Contains more than one Action-Verb)

3.1. IF Installation can not be deleted THEN System informs user about problems.

\section{TITLE: $\quad$ Edit client data}

GOAL: Edit client data

CONTEXT: Edit client data

ACTOR: user, System

RESOURCE: clients, database

EPISODES:

1. User select option for editing clients.

(Simplicity - TP: Missing Action-Verb in Present Tense)

2. User modifies personal client data.

3. System verifies correctness of data.

4. System saves a new client data to the database and informs user about it.

(Multiplicity - TP: ambiguous indicator)

(Simplicity - TP: Contains more than one Action-Verb)

EXCEPTION:

3.1. IF Data is incomplete or incorrect THEN System informs user about problems.

3.2. IF Client with same personal data already exists THEN System informs user about that fact. (Implicit - TP: ambiguous indicator)

4.1. IF Client data changes can't be saved THEN System informs user about reason why client can't be modified.

\section{TITLE: $\quad$ Edit contract}

GOAL: Edit contract

CONTEXT: Edit contract

ACTOR: User, System

RESOURCE: client, contract

EPISODES:

1. User select a client.

(Simplicity - TP: Missing Action-Verb in Present Tense)

2. User chooses option for editing an existing contract.

3. System displays transaction form.

4. User changes desired data.

5. System verifies information.

6. System saves changed contract.

EXCEPTION:

5.1. IF Data is incomplete or incorrect THEN System informs user about problems.

6.1. IF contract can't be saved THEN System informs user about that fact.

(Implicit - TP: ambiguous indicator)

TITLE:

\section{Edit installation}

GOAL: Edit installation

CONTEXT: Edit installation

ACTOR: User, System

RESOURCE: client, installation

EPISODES:

1. User select a client.

(Simplicity - TP: Missing Action-Verb in Present Tense)

2. User chooses option for editing an existing installation.

3. System displays installation form.

4. User changes desired data.

5. System verifies information.

6. System saves changed installation.

EXCEPTION: 
5.1. IF Data is incomplete or incorrect THEN System informs user about problems.

6.1. IF Installation can't be saved THEN System informs user about that fact.

(Implicit - TP: ambiguous indicator)

TITLE: Log in to the system

GOAL: $\log$ in to the system

CONTEXT: User Log in to the system

ACTOR: User, System

RESOURCE: main page, login option

EPISODES:

1. User selects login option.

2. User provides all required data.

(Quantifiable - TP: ambiguous indicator)

3. System verifies correctness of data.

4. System displays a main page.

EXCEPTION:

3.1. IF Data is incomplete or incorrect THEN System asks for data again.

TITLE: $\quad$ Prepare a report

GOAL: Prepare a report

CONTEXT: Prepare a report

ACTOR: User, System

RESOURCE: report, database, file

EPISODES:

1. User select option for creating reports.

(Simplicity - TP: Missing Action-Verb in Present Tense)

2. System displays a list of possible fields in the report.

3. User selects fields to be included in the report and rules to filter values from database.

(Multiplicity - TP: ambiguous indicator)

(Simplicity - TP: Contains more than one Action-Verb)

4. User order report generation.

(Simplicity - TP: Missing Action-Verb in Present Tense)

5. System asks for type and localisation of the output file with report.

(Multiplicity - FP: ambiguous indicator)

6 . User selects the type and localisation of the output file with report.

(Multiplicity - FP: ambiguous indicator)

7. System generates a report.

EXCEPTION:

7.1. IF Report can't be saved in given location THEN System displays information.

\section{TITLE: Request for licence}

GOAL: Request for licence

CONTEXT: Request for licence

ACTOR: User, System, PCSS Team Participant

RESOURCE: dLibra server, licence, contracts, file

EPISODES:

1. User select option for requesting a new licence.

(Simplicity - TP: Missing Action-Verb in Present Tense)

2. System displays the list of user's contracts.

3 . User selects one contract for licence request.

4. System contact with dLibra server to obtain all necessary data.

(Simplicity - TP: Missing Action-Verb in Present Tense)

(Quantifiable - TP: ambiguous indicator)

(Vagueness - TP: ambiguous indicator)

5. System validate given data.

(Simplicity - TP: Missing Action-Verb in Present Tense)

6. System store a request new licence and informs user about it.

(Multiplicity - TP: ambiguous indicator)

(Simplicity - TP: Contains more than one Action-Verb)

7. PCSS Team Participant approve request for a new licence. 
(Simplicity - TP: Missing Action-Verb in Present Tense)

8. User downloads the licence file.

\section{EXCEPTION:}

2.1. IF There are no contracts THEN System displays blank list.

3.1. IF selected contract can not have more licenses THEN System informs user about that fact. (Implicit - TP: ambiguous indicator)

5.1. IF Data is not valid THEN System informs user about incorrect data.

\section{TITLE: $\quad$ Search}

(Atomicity - TP: Missing Object)

(Soundness -FP: Title does not describe the Goal)

GOAL: Search

CONTEXT: Search

ACTOR: User, System

RESOURCE: filter, criteria, database, results

\section{EPISODES:}

1. User select option for searching.

(Simplicity - TP: Missing Action-Verb in Present Tense)

2. User selects subject of search (clients, contracts, installations).

3. System displays list of possible criteria.

4. User creates filter for searching.

(Simplicity - TP: Missing Object)

5. System search the database and displays the results.

(Multiplicity - TP: ambiguous indicator)

(Simplicity - TP: Contains more than one Action-Verb)

\section{EXCEPTION:}

4.1. IF Chosen criteria are invalid THEN System warns user.

5.1. IF No records found THEN System displays blank list. 


\section{A1.4 \\ Mobile News}

Table 49 shows the quantitative analysis in scenarios (Pre-conditions, Postconditions, Episodes, Exceptions) from Mobile News System, and how they are mapped in Petri-Net elements (Input places, Output places, Transitions).

Table 49 - Quantitative Analysis of Mobile News System

\begin{tabular}{|c|c|c|c|c|c|c|c|c|c|}
\hline $\begin{array}{c}\text { ID } \\
\text { Scenario }\end{array}$ & Scenario & $\begin{array}{l}\text { Num. Pre- } \\
\text { conditions/Conditions/ } \\
\text { Causes/Constraints }\end{array}$ & $\begin{array}{l}\text { Num. } \\
\text { Post- } \\
\text { conditions }\end{array}$ & $\begin{array}{l}\text { Num. } \\
\text { Episodes }\end{array}$ & $\begin{array}{l}\text { Num. } \\
\text { Exceptions }\end{array}$ & $\begin{array}{l}\text { Num. } \\
\text { Input } \\
\text { Places - } \\
\text { Petri-Net }\end{array}$ & $\begin{array}{l}\text { Num. } \\
\text { Transitions } \\
\text { - Petri-Net }\end{array}$ & $\begin{array}{l}\text { Num. } \\
\text { Output } \\
\text { Places } \\
\text { - Petri- } \\
\text { Net } \\
\end{array}$ & $\begin{array}{l}\text { Num. } \\
\text { Dummy } \\
\text { Places - } \\
\text { Petri- } \\
\text { Net }\end{array}$ \\
\hline 1 & Add a new channel & 0 & 0 & 12 & 1 & 0 & 13 & 0 & 15 \\
\hline 2 & $\begin{array}{l}\text { Add a new channel } \\
\text { group }\end{array}$ & 0 & 0 & 7 & 0 & 0 & 7 & 0 & 9 \\
\hline 3 & Configure the server & 0 & 0 & 4 & 1 & 0 & 5 & 0 & 7 \\
\hline 4 & $\begin{array}{ll}\begin{array}{l}\text { Configure } \\
\text { preferences }\end{array} & \text { user } \\
\end{array}$ & 0 & 0 & 4 & 0 & 0 & 4 & 0 & 6 \\
\hline 5 & Delete a channel & 0 & 0 & 10 & 0 & 0 & 10 & 0 & 12 \\
\hline 6 & $\begin{array}{lll}\begin{array}{l}\text { Delete } \\
\text { group }\end{array} & \text { a channel } \\
\end{array}$ & 0 & 0 & 7 & 0 & 0 & 7 & 0 & 9 \\
\hline 7 & Delete a user group & 0 & 0 & 7 & 1 & 0 & 8 & 0 & 10 \\
\hline 8 & Delete news & 0 & 0 & 2 & 0 & 0 & 2 & 0 & 4 \\
\hline 9 & Download news & 0 & 0 & 6 & 0 & 0 & 6 & 0 & 8 \\
\hline 10 & Post a group message & 0 & 0 & 4 & 0 & 0 & 4 & 0 & 6 \\
\hline 11 & Read news & 0 & 0 & 6 & 0 & 0 & 6 & 0 & 8 \\
\hline 12 & Register a new user & 1 & 0 & 5 & 2 & 1 & 7 & 0 & 9 \\
\hline 14 & $\begin{array}{l}\text { Subscribe/unsubscribe } \\
\text { news channels }\end{array}$ & 0 & 0 & 7 & 0 & 0 & 7 & 0 & 9 \\
\hline 15 & Update news & 0 & 0 & 5 & 0 & 0 & 5 & 0 & 7 \\
\hline & Total & 1 & 0 & 89 & 5 & 1 & 94 & 0 & 124 \\
\hline
\end{tabular}

Table 50 shows the Unambiguity analysis (qualitative) in scenarios (Title, Goal, Episodes, Exceptions) from Mobile News System. Let $\boldsymbol{T P}$ be the number of defects detected correctly by out analysis approach; $\boldsymbol{F P}$ be the number of defects detected incorrectly (defect does not occur); $\boldsymbol{F N}$ be the number of defects that are not detected (defect occurs).

Table 50 - Unambiguity Analysis of Mobile News System

\begin{tabular}{|c|c|c|c|c|c|c|c|c|c|c|c|c|c|c|c|c|c|c|c|c|c|}
\hline \multirow[t]{2}{*}{ Scenario } & \multicolumn{3}{|c|}{ Vague } & \multicolumn{3}{|c|}{ Subjective } & \multicolumn{3}{|c|}{ Optional } & \multicolumn{3}{|c|}{ Weak } & \multicolumn{3}{|c|}{ Multiple } & \multicolumn{3}{|c|}{ Implicit } & \multicolumn{3}{|c|}{ Quantifiable } \\
\hline & TP & FP & FN & TP & FP & FN & TP & FP & FN & TP & FP & FN & TP & FP & FN & TP & FP & FN & TP & FP & FN \\
\hline 1 & 1 & & & & & & & & & & & & 5 & & & 1 & & & 1 & & \\
\hline 2 & & & & & & & & & & & & & 4 & & & & & & & & \\
\hline 3 & & & & & & & & & & & & & 1 & & & & & & & & \\
\hline 4 & 1 & & & & & & & & & & & & 1 & & & & & & & & \\
\hline 5 & & & & & & & & & & & & & 3 & & & 2 & & & 2 & & \\
\hline 6 & & & & & & & & & & & & & 2 & & & 1 & & & 1 & & \\
\hline 7 & & & & & & & & & & & & & 1 & & & & & & 1 & & \\
\hline 8 & & & & & & & & & & & & & 1 & & & 1 & & & 1 & & \\
\hline 9 & & & & & & & & & & & & & 2 & & & 1 & & & 2 & & \\
\hline 10 & & & & & & & & & & & & & 1 & & & & & & & & \\
\hline 11 & 1 & & & & & & & & & & & & 1 & & & 1 & & & & & \\
\hline 12 & & & & & & & & & & & & & 1 & & & 4 & & & & & \\
\hline 13 & & & & & & & & & & & & & & & & & & & & & \\
\hline 14 & 2 & & & & & & & & & & & & 4 & & & 2 & & & & & \\
\hline 15 & 1 & & & & & & & & & & & & & 1 & & & & & & & \\
\hline Total & 6 & 0 & 0 & 0 & 0 & 0 & 0 & 0 & 0 & 0 & 0 & 0 & 27 & 1 & 0 & 13 & 0 & 0 & 8 & 0 & 0 \\
\hline
\end{tabular}

Table 51 shows the Completeness analysis (qualitative) in scenarios (Title, Goal, Episodes, Exceptions) from Mobile News System. Consistency is not 
shown because we do not have a baseline manually identified by Requirements Engineers.

Table 51 - Completeness Analysis of Mobile News System

\begin{tabular}{|c|c|c|c|c|c|c|c|c|c|c|c|c|c|c|c|c|c|c|c|c|c|c|c|c|}
\hline \multirow[t]{2}{*}{ ID Scenario } & \multicolumn{3}{|c|}{ Atomicity } & \multicolumn{3}{|c|}{ Simplicity } & \multicolumn{3}{|c|}{ Uniformity } & \multicolumn{3}{|c|}{ Usefulness } & \multicolumn{3}{|c|}{$\begin{array}{l}\begin{array}{l}\text { Conceptually } \\
\text { Soundness }\end{array} \\
\end{array}$} & \multicolumn{3}{|c|}{ Integrity } & \multicolumn{3}{|c|}{ Coherency } & \multicolumn{3}{|c|}{ Uniqueness } \\
\hline & TP & FP & $\mathbf{F N}$ & TP & FP & FN & TP & FP & FN & TP & FP & $\mathbf{F N}$ & TP & FP & FN & TP & FP & $\mathbf{F N}$ & TP & FP & FN & TP & $\mathbf{F P}$ & FN \\
\hline 1 & & & & 4 & & & 1 & & & 1 & & & & & & & & & & & & & & \\
\hline 2 & & & & 2 & & & & & & & & & & & & & & & & & & & & \\
\hline 3 & & & & 1 & & & 1 & & & & & & & & & & & & & & & & & \\
\hline 4 & & & & 2 & & & & & & & & & & & & & & & & & & & & \\
\hline 5 & & & & 2 & & & & & & 1 & & & & & & & & & & & & & & \\
\hline 6 & & & & 1 & & & & & & & & & & & & & & & & & & & & \\
\hline 7 & & & & & 1 & & 1 & & & & & & & & & & & & & & & & & \\
\hline 8 & & & & & & & & & & 1 & & & & & & & & & & & & & & \\
\hline 9 & & & & 1 & & & & & & & & & & & & & & & & & & & & \\
\hline 10 & & & & 1 & & & & & & & & & & & & & & & & & & & & \\
\hline 11 & & & & 1 & & & & & & & & & & & & & & & & & & & & \\
\hline 12 & & & & 5 & & & & 1 & & & & & & & & & & & & & & & & \\
\hline 13 & & & & 1 & & & & & & & & & & & & & & & & & & & & \\
\hline 14 & & & & 5 & & 1 & & & & & & & & & & & & & & & & & & \\
\hline 15 & & & & & & 1 & & & & & & & & & & & & & & & & & & \\
\hline Total & 0 & 0 & 0 & 26 & 1 & 2 & 3 & 1 & 0 & 3 & 0 & 0 & 0 & 0 & 0 & 0 & 0 & 0 & 0 & 0 & 0 & 0 & 0 & 0 \\
\hline
\end{tabular}

TITLE: Add a new channel

GOAL: Add a new channel

CONTEXT: Add a new channel

ACTOR: Administrator, System

RESOURCE: channel, service, channel group, database EPISODES:

(Usefulness - TP: Too long scenario - Num. episodes > 10)

1. Administrator logs on to the administration panel.

2. System displays administration options.

3. Administrator selects the Group and channel management option.

(Multiplicity - TP: ambiguous indicator)

4. System displays a list of defined channel groups and an add/delete group menu.

(Multiplicity - TP: ambiguous indicator)

5. Administrator chooses a group to which he wants to add a new channel.

(Simplicity - TP: Contains more than one Action-Verb)

(Implicit - TP: ambiguous indicator)

6. System displays a list of channels in the selected group and an add/delete menu.

(Multiplicity - TP: ambiguous indicator)

7. Administrator types the name of the channel and the URL of the news service and selects Add channel.

(Simplicity - TP: Contains more than one Action-Verb)

(Multiplicity - TP: ambiguous indicator)

8. System checks if a channel with the given name or URL has not been already defined and if so, inserts the channel information into a database.

(Multiplicity - TP: ambiguous indicator)

9. System adds an information about the new channel to a group message.

10. See step 6.

(Simplicity - TP: Missing Subject)

11. Administrator selects the Finish option.

12. System posts a group message containing information about all new channels in the selected channel group.

(Quantifiable - TP: ambiguous indicator)

\section{EXCEPTION:}

5.1. Administrator adds more channels. Proceed to step 7.

(Simplicity - TP: Contains more than one Sentence)

(Uniformity - TP: Incomplete cause)

(Vagueness - TP: ambiguous indicator)

TITLE: $\quad$ Add a new channel group

GOAL: Add a new channel group

CONTEXT: Add a new channel group 


\begin{abstract}
ACTOR: Administrator, System
RESOURCE: Group, channel, database EPISODES:
\end{abstract}

1. Administrator $\operatorname{logs}$ on to the administration panel.

2. System displays administration options.

3. Administrator selects the Group and channel management option.

(Multiplicity - TP: ambiguous indicator)

4. System displays a list of defined channel groups and an add/delete group menu.

(Multiplicity - TP: ambiguous indicator)

5. Administrator types the name of a new group and selects Add group.

(Simplicity - TP: Contains more than one Action-Verb)

(Multiplicity - TP: ambiguous indicator)

6. System checks if a group with the given name has not been already defined and if so, inserts the name of a new group into a database.

(Multiplicity - TP: ambiguous indicator)

7. See step 4.

(Simplicity - TP: Missing Subject)

TITLE: Configure the server

GOAL: Configure the server

CONTEXT: Configure the server

ACTOR: Administrator

RESOURCE: configuration settings

EPISODES:

1. Administrator logs on to the administration panel.

2. Administrator selects the Configure option.

3. Administrator chooses and changes the desired settings.

(Multiplicity - TP: ambiguous indicator)

(Simplicity - TP: Contains more than one Action-Verb)

4. Administrator saves configuration settings.

\title{
EXCEPTION:
}

4.1. Administrator cancels configuration changes.

(Uniformity - TP: Incomplete cause)

TITLE: Configure user preferences

GOAL: Configure user preferences

CONTEXT: Configure user preferences

ACTOR: User, System

RESOURCE: Preferences, options

\section{EPISODES:}

1. User chooses the Preferences option.

2. System displays a list of available options (i.e. font and color settings, local news caching, etc..).

(Vagueness - TP: ambiguous indicator)

3. User configures the option according to his/her preferences and confirm the changes.

(Multiplicity - TP: ambiguous indicator)

(Simplicity - TP: Contains more than one Action-Verb)

4. System saves user preferences configuration and displays main application view.

(Multiplicity - TP: ambiguous indicator)

(Simplicity - TP: Contains more than one Action-Verb)

TITLE:

Delete a channel

GOAL: Delete a channel

CONTEXT: Delete a channel

ACTOR: Administrator, System

RESOURCE: channel, channels, group, database

\section{EPISODES:}

(Usefulness - TP: Too long scenario - Num. episodes > 10)

1. Administrator logs on to the administration panel.

2. System displays administration options.

3. Administrator selects the Group and channel management option. 
(Multiplicity - TP: ambiguous indicator)

4. System displays a list of defined channel groups and an add/delete group menu.

(Multiplicity - TP: ambiguous indicator)

5. Administrator chooses a group containing the channel he wants to delete.

(Implicit - TP: ambiguous indicator)

(Simplicity - TP: Contains more than one Action-Verb)

6. System displays a list of channels in the selected group and an add/delete menu.

(Multiplicity - TP: ambiguous indicator)

7. Administrator selects the channel(s) he wants to delete and chooses the Delete option.

(Implicit - TP: ambiguous indicator)

(Simplicity - TP: Contains more than one Action-Verb)

8. System deletes the selected channels from the database.

9. System posts a group message containing information about the deleted channels in the selected channel group to all users involved (subscribing the deleted channels).

(Quantifiable - TP: ambiguous indicator)

10. System deletes all subscription information concerning the deleted channels.

(Quantifiable - TP: ambiguous indicator)

TITLE: Delete a channel group

GOAL: Delete a channel group

CONTEXT: Delete a channel group

ACTOR: Administrator, System

RESOURCE: channel group

EPISODES:

1. Administrator logs on to the administration panel.

2. System displays administration options.

3. Administrator selects the Group and channel management option.

(Multiplicity - TP: ambiguous indicator)

4. System displays a list of defined channel groups and an add/delete group menu.

(Multiplicity - TP: ambiguous indicator)

5. Administrator selects the group(s) he wants to delete and chooses the Delete option.

(Simplicity - TP: Contains more than one Action-Verb)

6. System asks for confirmation.

(Implicit - TP: ambiguous indicator)

7. System deletes all channels from the selected groups (see: UC5, steps 8 to 10).

(Quantifiable - TP: ambiguous indicator)

\section{TITLE: Delete a user group}

GOAL: Delete a user group

CONTEXT: Delete a user group

ACTOR: Administrator, System

RESOURCE: users, accounts

EPISODES:

1. Administrator logs on to the administration panel.

2. System displays administration options.

3. Administrator selects the Delete users option.

4. System displays the users deletion menu.

5. Administrator selects deletion options (i.e. date of users` last login).

6. Administrator confirms deletion request.

7. System finds all users matching deletion criteria and deletes found user accounts.

(Simplicity - FN: Contains more than one Action-Verb)

(Quantifiable - TP: ambiguous indicator)

(Multiplicity - TP: ambiguous indicator)

EXCEPTION:

6.1. Administrator cancels user deletion.

(Uniformity - TP: Incomplete cause)

TITLE: Delete news

GOAL: Delete news

CONTEXT: Delete news 


\author{
ACTOR: System \\ RESOURCE: messages, database \\ EPISODES:
}

(Usefulness - TP: Too short scenario - Num. episodes $<3$ )

1. System queries the database for news messages, whose expiry date and time have passed.

(Implicit - TP: ambiguous indicator)

(Multiplicity - TP: ambiguous indicator)

2. System deletes all returned messages from the database.

(Quantifiable - TP: ambiguous indicator)

TITLE: Download news

GOAL: Download news

CONTEXT: Download news

ACTOR: User, system

RESOURCE: news, server, messages, channels, database

\title{
EPISODES:
}

1. User chooses to update locally stored news.

2. System sends a HTTP request to the Mobile News server.

3. Server sends all pending group messages.

(Quantifiable - TP: ambiguous indicator)

4. Server sends separate news messages from all subscribed channels.

(Quantifiable - TP: ambiguous indicator)

5. System receives news messages and stores them in a local database.

(Multiplicity - TP: ambiguous indicator)

(Implicit - TP: ambiguous indicator)

(Simplicity - TP: Contains more than one Action-Verb)

6. System displays a list of groups with subscribed channels and the number of new messages in each of them.

(Multiplicity - TP: ambiguous indicator)

TITLE: $\quad$ Post a group message

GOAL: Post a group message

CONTEXT: Post a group message

ACTOR: Administrator, User

RESOURCE: message, data

\section{EPISODES:}

1. Administrator logs on to the administration panel.

2. Administrator selects the Post group message option.

3. Administrator types the message and posts it.

(Multiplicity - TP: ambiguous indicator)

(Simplicity - TP: Contains more than one Action-Verb)

4. User receives the message when downloading new data.

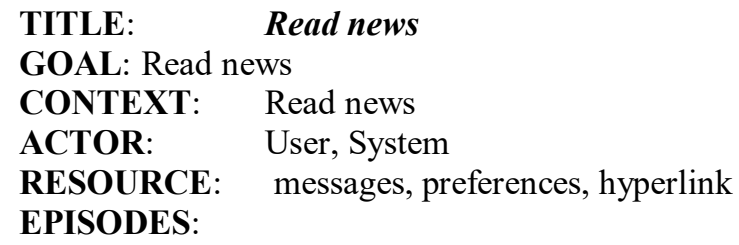

1. User chooses a news group from the Today menu.

2. System displays a list of topics of available messages in chosen group.

(Vagueness - TP: ambiguous indicator)

3. User chooses a topic.

4. System displays the message using user's appearance preferences.

5. User reads the message and closes it or uses a hyperlink to go to the full message.

(Multiplicity - TP: ambiguous indicator)

(Simplicity - TP: Contains more than one Action-Verb)

(Implicit - TP: ambiguous indicator)

6. System marks the message as Read. 
TITLE: Register a new user

GOAL: Register a new user

CONTEXT: Register a new user

ACTOR: User, System

RESOURCE: server, user account, ID, preferences

EPISODES:

1. System asks the user if he/she wants to register.

(Implicit - TP: ambiguous indicator)

(Simplicity - TP: Contains more than one Action-Verb)

2. User confirms he/she wants to register.

(Implicit - TP: ambiguous indicator)

(Simplicity - TP: Contains more than one Action-Verb)

3. System sends a registration request to the server.

4. Server creates a new user account and sends back a user ID.

(Multiplicity - TP: ambiguous indicator)

(Simplicity - TP: Contains more than one Action-Verb)

5. System stores the user ID and instructs the user how to subscribe news channels or configure his/her preferences.

(Implicit - TP: ambiguous indicator)

(Simplicity - TP: Contains more than one Action-Verb)

EXCEPTION:

2.1. IF User refuses to register THEN System displays an information that it cannot be used without prior registration.

(Implicit - TP: ambiguous indicator)

2.1.2 User confirms the message.

(Simplicity - TP: Nested Exception Solution must be treated by a scenario)

(Uniformity - FP: Incomplete cause)

\section{TITLE: Run the application}

GOAL: Run the application

CONTEXT: Run the application

ACTOR: User, System

RESOURCE: application, channels

\section{EPISODES:}

1. User starts the application.

2. System checks for registration information.

3. IF the user is already registered THEN the system automatically updates news messages from subscribed channels (refer to Download news use case). If no, the system attempts to Register a new user (refer to Register a new user use case).

(Simplicity - TP: Contains more than one Action-Verb)

TITLE: $\quad$ Subscribe/unsubscribe news channels

GOAL: Subscribe/unsubscribe news channels

CONTEXT: Subscribe/unsubscribe news channels

ACTOR: User, System

RESOURCE: server, channels, database

EPISODES:

1. User chooses the Channel subscription option.

2. System requests for and downloads a list of available groups and channels.

(Multiplicity - TP: ambiguous indicator)

(Vagueness- TP: ambiguous indicator)

(Simplicity - TP: Contains more than one Action-Verb)

3. System displays a tree view of available groups and channels and marks those already subscribed by the user.

(Multiplicity - TP: ambiguous indicator)

(Vagueness- TP: ambiguous indicator)

(Simplicity - TP: Contains more than one Action-Verb)

4. User selects the channels he/she wants to subscribe and/or deselects already subscribed channels to unsubscribe them and chooses the Change subscription options.

(Implicit - TP: ambiguous indicator) 
(Simplicity - TP: Contains more than one Action-Verb)

5. System sends the subscription configuration to the Mobile News server and waits for confirmation.

(Multiplicity - TP: ambiguous indicator)

(Simplicity - TP: Contains more than one Action-Verb)

6. Server alters the user's subscription configuration in a database and sends a change confirmation.

(Multiplicity - TP: ambiguous indicator)

(Simplicity - TP: Contains more than one Action-Verb)

7. System receives the confirmation and displays it.

(Implicit - TP: ambiguous indicator)

(Simplicity - FN: Contains more than one Action-Verb)

TITLE: Update news

GOAL: Update news

CONTEXT: Update news

ACTOR: Daemon, System

RESOURCE: service, file, message, database

EPISODES:

1. Daemon sends a HTTP request to a defined news service.

2. System receives a RSS-like formatted news file.

3. System parses the received news file.

4. System assigns an expiry date and time to each incoming message.

(Simplicity - FP: Contains more than one Action-Verb)

(Multiplicity - FP: ambiguous indicator)

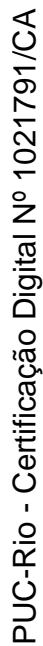

5. System inserts appropriate parts of the news file into a news database.

(Vagueness - TP: ambiguous indicator) 


\title{
Appendix A2 \\ Quality Models of Related Work
}

\author{
A.2.1. \\ Static Analysis of Software Requirements Specification
}

A.2.1.1.
Static Analysis of Requirement Statements

Table 52 - Quality Indicators of ARM (Wilson et al., 1997)

\begin{tabular}{|c|c|c|c|}
\hline Property & \multicolumn{2}{|l|}{ Description } & Indicators \\
\hline Imperatives & \multicolumn{2}{|l|}{$\begin{array}{l}\text { The sentence contains words or phrases that command } \\
\text { that something must be provided. }\end{array}$} & $\begin{array}{l}\text { shall, must, must not, is required } \\
\text { to, are applicable. }\end{array}$ \\
\hline Continuances & \multicolumn{2}{|l|}{$\begin{array}{l}\text { The sentence contains phrases that follow an } \\
\text { imperative and introduce the SRS at a lower level. }\end{array}$} & $\begin{array}{l}\text { below: , as follows: , following: } \\
\text { listed:: }\end{array}$ \\
\hline Directives & \multicolumn{2}{|l|}{$\begin{array}{l}\text { The sentence contains words or phrases that point to } \\
\text { illustrative information within the SRS. }\end{array}$} & figure, table, for example, note: \\
\hline Options & \multicolumn{2}{|l|}{$\begin{array}{l}\text { The sentence contains words that give the developer } \\
\text { latitude in satisfying the specification statements that } \\
\text { contain them. }\end{array}$} & can , may, optionally \\
\hline Weak Phrases & \multicolumn{2}{|l|}{$\begin{array}{l}\text { The sentence contains clauses that are apt to cause } \\
\text { uncertainty and leave room for multiple } \\
\text { interpretations. }\end{array}$} & $\begin{array}{l}\text { adequate, as a minimum, as } \\
\text { applicable, easy, as appropriate }\end{array}$ \\
\hline Readability & \multicolumn{2}{|l|}{$\begin{array}{l}\text { It measures the difficulty in reading the Document or a } \\
\text { sentence. } \\
\text { This metric is the Coleman-Liau Formula readability } \\
\text { metric: } \quad(5.89 * \\
0.3 * \text { sentences } /(100 * \text { words })-15.8])\end{array}$} & $\begin{array}{l}\text { If it is }>55.8 \text { the document is } \\
\text { difficult -to-read. }\end{array}$ \\
\hline \multicolumn{4}{|c|}{ Table 53 - Expressiveness Quality Model of QuARS (Gnesi et al., 2005) } \\
\hline Property & Description & \multicolumn{2}{|c|}{ Indicators } \\
\hline Vagueness & $\begin{array}{l}\text { The sentence contains words or phrases having a non } \\
\text { uniquely quantifiable meaning. }\end{array}$ & \multicolumn{2}{|r|}{$\begin{array}{l}\text { clear, easy, strong, good, bad, efficient, } \\
\text { useful, significant }\end{array}$} \\
\hline Subjectivity & $\begin{array}{l}\text { The sentence contains words or phrases expressing } \\
\text { personal opinions or feeling. }\end{array}$ & \multicolumn{2}{|c|}{$\begin{array}{l}\text { similar, better, similarly, worse, } \\
\text { having in mind, take into account }\end{array}$} \\
\hline Optionality & $\begin{array}{l}\text { The sentence contains words or phrases expressing an } \\
\text { optional part (i.e. a part that can or cannot be considered). }\end{array}$ & \multicolumn{2}{|c|}{$\begin{array}{l}\text { possibly, eventually, if case, if } \\
\text { possible, if appropriate, if needed }\end{array}$} \\
\hline Implicitly & $\begin{array}{l}\text { The sentence does not specify the subject or object by } \\
\text { means of its specific name but uses pronoun or other } \\
\text { indirect reference. }\end{array}$ & \multicolumn{2}{|c|}{$\begin{array}{l}\text { this, these, that, those, it, they, } \\
\text { previous, next, following, below }\end{array}$} \\
\hline Weakness & $\begin{array}{l}\text { The sentence contains a weak verb. A verb that makes the } \\
\text { sentence not imperative is considered weak. }\end{array}$ & \multicolumn{2}{|c|}{ can, could, may, ... } \\
\hline $\begin{array}{l}\text { Under- } \\
\text { specification }\end{array}$ & $\begin{array}{l}\text { The sentence contains a word identifying a class of objects } \\
\text { without a modifier specifying an instance of this class }\end{array}$ & \multicolumn{2}{|c|}{$\begin{array}{l}\text { flow instead of data flow, control flow, } \\
. . \quad \text { testing instead of functional } \\
\text { testing, unit testing }\end{array}$} \\
\hline Multiplicity & $\begin{array}{l}\text { The sentence has more than one main verb, subject or } \\
\text { object }\end{array}$ & \multicolumn{2}{|r|}{ and, or, and/or, ... } \\
\hline Readability & $\begin{array}{l}\text { It measures the difficulty in reading the Document or a } \\
\text { sentence. } \\
\text { This metric is the Coleman-Liau Formula readability } \\
\text { metric: ( } 5.89^{*} \text { letters/words- } 0.3 * \text { sentences/(100*words)- } \\
15.8])\end{array}$ & \multicolumn{2}{|c|}{$\begin{array}{l}\text { If it is }>15 \text { the document is difficult - } \\
\text { to-read. }\end{array}$} \\
\hline
\end{tabular}


Table 54 - Ambiguity Indicators of SRRE (Tjong, 2008)

\begin{tabular}{|c|c|c|}
\hline Property & Description & Indicators \\
\hline Continuance & $\begin{array}{l}\text { The sentence introduces further } \\
\text { specification }\end{array}$ & as follows, below, following, in addition, in particular \\
\hline Coordinator & $\begin{array}{l}\text { The sentence introduces a } \\
\text { coordination ambiguity }\end{array}$ & and, and/or, or \\
\hline Directive & $\begin{array}{l}\text { The sentence introduces extra } \\
\text { information }\end{array}$ & e.g., etc., figure, for example, i.e., note, table \\
\hline Incomplete & $\begin{array}{l}\text { The sentence introduces } \\
\text { information that are not in SRS }\end{array}$ & $\begin{array}{l}\text { as a minimum, as defined, as specified, in addition, is defined, } \\
\text { no practical limit }\end{array}$ \\
\hline Optional & $\begin{array}{l}\text { The sentence expresses an optional } \\
\text { part }\end{array}$ & as desired, at last, either, eventually, if appropriate \\
\hline Pronoun & $\begin{array}{l}\text { The sentence uses pronouns or } \\
\text { indirect reference }\end{array}$ & $\begin{array}{l}\text { anyone, anybody, anything i, it, its, itself, me, mine, most, my, } \\
\text { myself, nobody, none, no one, nothing, our, ours, ourselves, } \\
\text { she, someone, somebody, yourselves }\end{array}$ \\
\hline Plural & The sentence contains plural words & $\begin{array}{l}\text { The Plural corpus contains a list of } 11,287 \text { plural nouns, each } \\
\text { ending in s }\end{array}$ \\
\hline Quantifier & $\begin{array}{l}\text { The sentence introduces terms } \\
\text { used for quantification }\end{array}$ & all, any, few, little, many, much, several, some \\
\hline Vague & $\begin{array}{l}\text { The sentence introduces terms that } \\
\text { contribute vagueness }\end{array}$ & $\begin{array}{l}l,<>,(),[],\{\}, ;, ?, !, \text { adaptability, additionally, adequate, } \\
\text { aggregate, also, ancillary, arbitrary, appropriate, as } \\
\text { appropriate varying }\end{array}$ \\
\hline Weak & The sentence contains a weak verb & $\begin{array}{l}\text { can, could, may, might, ought to, preferred, should, will, } \\
\text { would }\end{array}$ \\
\hline
\end{tabular}

Table 55 - Requirements language criteria (IEEE, 2011; Femmer et al., 2014)

\begin{tabular}{|l|l|l|}
\hline Smell & Description & Indicators \\
\hline $\begin{array}{l}\text { Ambiguous Adverbs and } \\
\text { Adjectives }\end{array}$ & $\begin{array}{l}\text { Refer to adverbs and adjectives that are } \\
\text { unspecific }\end{array}$ & almost always, significant, minimal \\
\hline Vague Pronouns & Are unclear relations of a pronoun & Using Part-of-speech Tagging \\
\hline Subjective Language & $\begin{array}{l}\text { Refer to words of which the semantics is not } \\
\text { objective }\end{array}$ & $\begin{array}{l}\text { User friendly, easy to use, cost } \\
\text { effective }\end{array}$ \\
\hline Comparative Phrases & $\begin{array}{l}\text { Are used in requirements that express a } \\
\text { relation of the system to specific other systems }\end{array}$ & Using Morphological Analysis \\
\hline Superlatives & $\begin{array}{l}\text { Are used in requirements that express a } \\
\text { relation of the system to all other systems }\end{array}$ & $\begin{array}{l}\text { Using Morphological Analysis and } \\
\text { Part-of-speech Tagging }\end{array}$ \\
\hline Negative Statements & $\begin{array}{l}\text { Are "statements of system capability not to be } \\
\text { provided" }\end{array}$ & must not \\
\hline $\begin{array}{l}\text { Open-ended, Non-verifiable } \\
\text { Terms }\end{array}$ & $\begin{array}{l}\text { Are hard to verify as they offer a choice of } \\
\text { possibilities }\end{array}$ & $\begin{array}{l}\text { Provide support, but not limited to, as } \\
\text { a minimum }\end{array}$ \\
\hline Loopholes & $\begin{array}{l}\text { Enable stakeholders to ignore certain parts of } \\
\text { the application }\end{array}$ & $\begin{array}{l}\text { Is possible, as appropriate, as } \\
\text { applicable }\end{array}$ \\
\hline Incomplete References & Are references that a reader cannot follow & Not implemented \\
\hline
\end{tabular}

Table 56 - Potentially problematic constructs (from Berry et al., 2012)

\begin{tabular}{|c|c|c|}
\hline Smell & Description & Indicators \\
\hline Warn_AND & The "and" conjunction can imply several meanings, & and \\
\hline Warn_OR & $\begin{array}{l}\text { The "or" conjunction can imply "exclusive or", or "inclusive } \\
\text { or". }\end{array}$ & or \\
\hline Warn_Quantifier & Terms used for quantification & all, any, every \\
\hline Warn_Pronoun & Pronouns can lead to referential ambiguity. & they \\
\hline Warn_VagueTerms & $\begin{array}{l}\text { There are several vague terms that are commonly used in } \\
\text { requirements documents. }\end{array}$ & $\begin{array}{l}\text { user-friendly, support, } \\
\text { acceptable, up to, } \\
\text { periodically }\end{array}$ \\
\hline Warn_PassiveVoice & $\begin{array}{l}\text { Passive voice blurs the actor of the requirement and must be } \\
\text { avoided in requirements. }\end{array}$ & it \\
\hline $\begin{array}{l}\text { Warn_Complex_Sente } \\
\text { nce }\end{array}$ & $\begin{array}{l}\text { Using multiple conjunctions in the same requirements } \\
\text { sentence make the sentence hard to read and are likely to } \\
\text { cause ambiguity. }\end{array}$ & and, or \\
\hline Warn_Plural_Noun & Plural Nouns can potentially lead to ambiguous situations & \\
\hline $\begin{array}{l}\text { Warn_Adverb_in_Ver } \\
\text { b_Phrase }\end{array}$ & $\begin{array}{l}\text { Adverbial verb phrases are discouraged due to vagueness and } \\
\text { the chances of important details remaining tacit in the adverb }\end{array}$ & periodically \\
\hline $\begin{array}{l}\text { Warn_Adj_followed_b } \\
\text { y_Conjunction }\end{array}$ & $\begin{array}{l}\text { The adjective followed by two nouns separated by a } \\
\text { conjunction, can lead to ambiguity due to the possible } \\
\text { relation of adjective with just first noun or both nouns. }\end{array}$ & compliant \\
\hline
\end{tabular}


Table 57 - Quality User Story Framework (Lucassen et al., 2015)

\begin{tabular}{|c|c|c|}
\hline Quality & Criteria & Description \\
\hline \multirow{3}{*}{$\begin{array}{l}\text { Syntactic: quality, concerning } \\
\text { the textual structure of a user } \\
\text { story without considering its } \\
\text { meaning. }\end{array}$} & Atomic & A user story expresses a requirement for exactly one feature \\
\hline & Minimal & $\begin{array}{l}\text { A user story contains nothing more than role, means and } \\
\text { ends }\end{array}$ \\
\hline & Well-formed & A user story includes at least a role and a means \\
\hline \multirow{4}{*}{$\begin{array}{l}\text { Semantic: quality, concerning } \\
\text { the relations and meaning of } \\
\text { (parts of) the user story text. }\end{array}$} & Conflict-free & $\begin{array}{l}\text { A user story should not be inconsistent with any other user } \\
\text { story }\end{array}$ \\
\hline & $\begin{array}{l}\text { Conceptually } \\
\text { sound }\end{array}$ & $\begin{array}{l}\text { The means expresses a feature and the ends expresses a } \\
\text { rationale, not something else }\end{array}$ \\
\hline & $\begin{array}{l}\text { Problem- } \\
\text { oriented }\end{array}$ & A user story only specifies the problem, not the solution to it \\
\hline & Unambiguous & $\begin{array}{l}\text { A user story avoids terms or abstractions that may lead to } \\
\text { multiple interpretations }\end{array}$ \\
\hline \multirow{7}{*}{$\begin{array}{l}\text { Pragmatic: quality, regarding } \\
\text { choosing the most effective } \\
\text { alternatives for communicating } \\
\text { a given set of requirements. }\end{array}$} & Complete & $\begin{array}{l}\text { Implementing a set of user stories creates a feature-complete } \\
\text { application, no steps are missing }\end{array}$ \\
\hline & $\begin{array}{l}\text { Explicit } \\
\text { dependencies }\end{array}$ & $\begin{array}{l}\text { Link all unavoidable, non-obvious dependencies on user } \\
\text { stories }\end{array}$ \\
\hline & Full sentence & A user story is a well-formed full sentence \\
\hline & Independent & $\begin{array}{l}\text { The user story is self-contained, avoiding inherent } \\
\text { dependencies on other user stories }\end{array}$ \\
\hline & Scalable & $\begin{array}{l}\text { User stories do not denote too coarse-grained requirements } \\
\text { that are difficult to plan and prioritize }\end{array}$ \\
\hline & Uniform & All user stories follow roughly the same template \\
\hline & Unique & Every user story is unique, duplicates are avoided \\
\hline
\end{tabular}

A.2.1.2.

\section{Static Analysis of Scenarios}

\section{Table 58 - Taxonomy of defects in use case models (Anda and Sjoberg, 2002)}

\begin{tabular}{|c|c|c|}
\hline \multicolumn{2}{|l|}{ Checklist Element } & Description \\
\hline \multirow{2}{*}{$\begin{array}{l}\text { Use case } \\
\text { diagram: check } \\
\text { the } \\
\text { completeness } \\
\text { and consistency } \\
\text { in use case } \\
\text { diagrams }\end{array}$} & Actor & $\begin{array}{l}\text { Human users or external entities that will interact with the system are not identified. } \\
\text { Incorrect description of actors or wrong connection between actor and use case. } \\
\text { Description of actor is inconsistent with its behavior in use cases. } \\
\text { Too broadly defined actors or ambiguous description of actor. } \\
\text { Actors that do not derive value from/provide value to the system. }\end{array}$ \\
\hline & Use case & $\begin{array}{l}\text { Required functionality is not described in use cases. Actors have goals that do not } \\
\text { have corresponding use cases. } \\
\text { Incorrect description of a use case } \\
\text { Description is inconsistent with reaching the goal of the use case. } \\
\text { Name of use case does not reflect the goal of the use case. } \\
\text { Use cases with functionality outside the scope of the system or use cases that } \\
\text { duplicate functionality. }\end{array}$ \\
\hline \multirow{4}{*}{$\begin{array}{l}\text { Use case } \\
\text { description: } \\
\text { check the } \\
\text { completeness } \\
\text { and consistency } \\
\text { in use cases and } \\
\text { their } \\
\text { relationships }\end{array}$} & Flow of events & $\begin{array}{l}\text { Input or output for use cases is not described. Events that are necessary for } \\
\text { understanding the use cases are missing. } \\
\text { Incorrect description of one or several events. } \\
\text { Events that are inconsistent with reaching the goal of the use case they are part of. } \\
\text { Ambiguous description of events, perhaps because of too little detail. } \\
\text { Superfluous steps or too much detail in steps. }\end{array}$ \\
\hline & Variations & $\begin{array}{l}\text { Variations that may occur when attempting to achieve the goal of a use case are not } \\
\text { specified. } \\
\text { Incorrect description of a variation. } \\
\text { Variations that are inconsistent with the goal of the use case. } \\
\text { Ambiguous description of what leads to a particular variation. } \\
\text { Variations that are outside the scope of the system. }\end{array}$ \\
\hline & $\begin{array}{l}\text { Relation } \\
\text { between use } \\
\text { cases }\end{array}$ & $\begin{array}{l}\text { Common functionality is not separated out in included use cases. } \\
\text { Inconsistencies between diagram and descriptions, inconsistent terminology, } \\
\text { inconsistencies between use cases, or different level of granularity. }\end{array}$ \\
\hline & $\begin{array}{l}\text { Trigger, pre- } \\
\text { condition and } \\
\text { post-condition }\end{array}$ & $\begin{array}{l}\text { Trigger, pre- or post-conditions have been omitted. } \\
\text { Incorrect assumptions or results have led to incorrect pre- or post- conditions. } \\
\text { Pre- or post- conditions are inconsistent with goal or flow of events. } \\
\text { Ambiguous description of trigger, pre- or post-condition } \\
\text { Superfluous trigger, pre-or post-conditions. }\end{array}$ \\
\hline
\end{tabular}


Table 59 - Scenario Checklist (Leite et al., 2000; Leite et al., 2005)

\begin{tabular}{|c|c|c|}
\hline \multicolumn{2}{|l|}{ Checklist Element } & \multirow[b]{2}{*}{$\begin{array}{l}\text { Description } \\
\text { Check the existence of more than one episode per } \\
\text { scenario; } \\
\text { Check the syntax of each scenario element as } \\
\text { established in the scenario model; }\end{array}$} \\
\hline \multirow{3}{*}{$\begin{array}{l}\text { Intra-scenario: verify } \\
\text { each component in } \\
\text { every scenario to } \\
\text { confirm its consistency } \\
\text { with the components } \\
\text { and adherence to the } \\
\text { scenario model }\end{array}$} & $\begin{array}{l}\text { Syntactic } \\
\text { verification }\end{array}$ & \\
\hline & $\begin{array}{l}\text { Relationship among } \\
\text { components }\end{array}$ & $\begin{array}{l}\text { Check that every Actor participates in at least one } \\
\text { episode; } \\
\text { Check that every Actor mentioned in episodes is } \\
\text { included in the Actor element; } \\
\text { Check that every Resource is used in at least one } \\
\text { episode; } \\
\text { Check that every Resource mentioned in episodes is } \\
\text { included in the Resource element; }\end{array}$ \\
\hline & $\begin{array}{l}\text { Semantic } \\
\text { verification }\end{array}$ & $\begin{array}{l}\text { Check the coherence between the Title and the Goal; } \\
\text { Ensure that the set of Episodes satisfies the Goal and is } \\
\text { within the Context; } \\
\text { Ensure that actions presents in the Pre-conditions are } \\
\text { already performed; } \\
\text { Ensure that Episodes contain only actions to be } \\
\text { performed; }\end{array}$ \\
\hline \multirow[t]{2}{*}{ 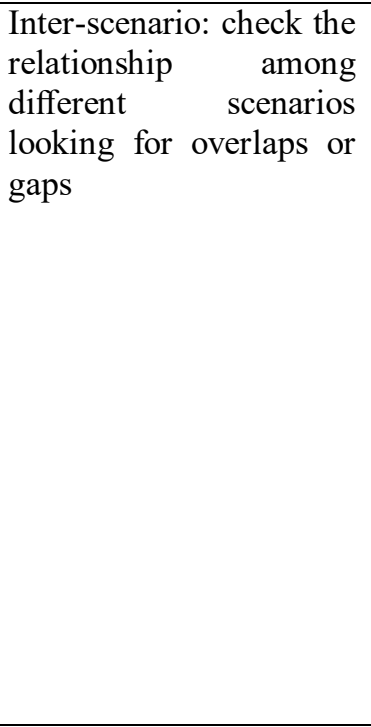 } & $\begin{array}{l}\text { Scenario } \\
\text { relationship }\end{array}$ & $\begin{array}{l}\text { Check that every Episode identified as sub-scenario } \\
\text { exists within the set of scenarios; } \\
\text { Check that the set of Episodes of every sub-scenario is } \\
\text { not already included in another scenario; } \\
\text { Check that every Exception is treated by a scenario; } \\
\text { Check that every Pre-condition is either an } \\
\text { uncontrollable fact or is satisfied by another scenario; } \\
\text { Check coherence between related scenario Pre- } \\
\text { conditions and scenario Pre-conditions; } \\
\text { Check that geographical and Temporal location of } \\
\text { related scenarios are equal or more restricted than } \\
\text { those of scenario; }\end{array}$ \\
\hline & Scenario overlap & $\begin{array}{l}\text { Check that Goal coincidence only takes place in } \\
\text { different situations; } \\
\text { Check that Episode coincidence only takes place in } \\
\text { different situations; } \\
\text { Check that Context coincidence only takes place in } \\
\text { different situations; }\end{array}$ \\
\hline $\begin{array}{l}\text { LEL Coverage: ensure } \\
\text { that LEL symbols are } \\
\text { properly used and that } \\
\text { every phrase } \\
\text { emphasized as a LEL } \\
\text { symbol is actually part } \\
\text { of LEL. }\end{array}$ & \multicolumn{2}{|c|}{$\begin{array}{l}\text { Check that every lexicon symbol is identified; } \\
\text { Check the correct use of lexicon symbols; } \\
\text { Check that Actors are preferentially Subject symbols; } \\
\text { Check that Resources are preferentially Object symbols; } \\
\text { Check that the behavioral response of Subject symbols are covered by } \\
\text { scenarios; }\end{array}$} \\
\hline
\end{tabular}


Table 60 - The 7Cs Verification Heuristics (Phalp et al., 2007)

\begin{tabular}{|c|c|c|}
\hline \multicolumn{2}{|l|}{ Property } & \multirow{2}{*}{$\begin{array}{l}\text { Description } \\
\text { The use case should contain all that is required to answer } \\
\text { the problem. }\end{array}$} \\
\hline \multirow[t]{2}{*}{ Coverage } & Scope & \\
\hline & Span & $\begin{array}{l}\text { The use case should only contain detail relevant to the } \\
\text { problem statement. Extra unnecessary information } \\
\text { provided is out of problem scope and not required. }\end{array}$ \\
\hline \multirow[t]{3}{*}{ Cogent } & Text Order & $\begin{array}{l}\text { The use case should follow a logical path with events in the } \\
\text { description in the correct order. }\end{array}$ \\
\hline & Dependencies & $\begin{array}{l}\text { The use case should complete as an end-to-end transaction } \\
\text { (which can include alternative/exceptional flows). Does the } \\
\text { actor reach a state that stops the transaction from } \\
\text { terminating as expected? }\end{array}$ \\
\hline & Rational Answer & $\begin{array}{l}\text { The logic of the use case description should provide a } \\
\text { plausible answer to the problem. }\end{array}$ \\
\hline Coherent & \multicolumn{2}{|c|}{$\begin{array}{l}\text { The sentence being written should repeat a noun in the last sentence or a previous } \\
\text { sentence, if possible. The description is easier to read and quicker to understand if } \\
\text { there is logical coherence throughout. }\end{array}$} \\
\hline $\begin{array}{l}\text { Consistent } \\
\text { Abstraction }\end{array}$ & \multicolumn{2}{|c|}{$\begin{array}{l}\text { The use case should be at a consistent level of abstraction through- out. Mixing } \\
\text { abstraction levels (problem domain, interface specification, internal design mixes) } \\
\text { may cause difficulty in understanding. }\end{array}$} \\
\hline \multirow[t]{2}{*}{$\begin{array}{l}\text { Consistent } \\
\text { Structure }\end{array}$} & Variations & $\begin{array}{l}\text { Alternative paths should be excluded from the main flow. } \\
\text { Inclusion of alternative paths in the main flow reduces } \\
\text { readability. }\end{array}$ \\
\hline & Sequence & Numbering of events in the main flow should be consistent. \\
\hline $\begin{array}{l}\text { Consistent } \\
\text { Grammar }\end{array}$ & \multicolumn{2}{|c|}{$\begin{array}{l}\text { Simple present tense should be used throughout. Adverbs, adjectives, pronouns, } \\
\text { synonyms and negatives should be avoided. }\end{array}$} \\
\hline \multirow[t]{3}{*}{$\begin{array}{l}\text { Consideration of } \\
\text { Alternatives }\end{array}$} & Separation & $\begin{array}{l}\begin{array}{l}\text { There should be a separate section for any } \\
\text { alternative/exceptional paths to the main flow. }\end{array} \\
\end{array}$ \\
\hline & Viable & Alternatives should be viable and make sense. \\
\hline & Numbering & $\begin{array}{l}\text { Alternative numberings should exactly match the numbers } \\
\text { in the main flow. }\end{array}$ \\
\hline
\end{tabular}


Table 61 - The Use Case Defects (Ciemniewska and Jurkiewicz, 2007)

\begin{tabular}{|c|c|c|}
\hline Level & Defect & Description \\
\hline $\begin{array}{l}\text { Specification-Level } \\
\text { Bad Smells }\end{array}$ & $\begin{array}{l}\text { Use-Case } \\
\text { Duplication }\end{array}$ & $\begin{array}{l}\text { At the level of requirements specification, where there are } \\
\text { many use cases, a quite common defect which we have } \\
\text { observed is Use-Case Duplication. }\end{array}$ \\
\hline \multirow[t]{4}{*}{ Use-Case Level } & $\begin{array}{l}\text { Too long or too short } \\
\text { use cases }\end{array}$ & $\begin{array}{l}\text { It is strongly recommended to keep use cases 3-9 steps } \\
\text { long. Too long use cases are difficult to read and } \\
\text { understand. Too short use cases, consisting of one or two } \\
\text { steps, distract a reader from the context and, as well, } \\
\text { make the specification more difficult to understand. }\end{array}$ \\
\hline & $\begin{array}{l}\text { Complicated } \\
\text { extension }\end{array}$ & $\begin{array}{l}\text { When the interruption causes the execution of a } \\
\text { repeatable, consistent sequence of steps, then this } \\
\text { sequence should be extracted to a separate use case }\end{array}$ \\
\hline & $\begin{array}{l}\text { Repeated actions in } \\
\text { neighbouring steps }\end{array}$ & $\begin{array}{l}\text { Every step of a use case should represent one particular } \\
\text { action. The action may consist of one or more moves } \\
\text { which can be taken as an integrity. Every step should } \\
\text { contain significant information which rather reflect user } \\
\text { intent then a single move. Splitting these movements into } \\
\text { separate steps may lead to long use cases, bothersome to } \\
\text { read and hard to maintain. }\end{array}$ \\
\hline & Inappropriate naming & $\begin{array}{l}\text { Every use case should have a descriptive name. The title } \\
\text { of each use case presents a goal that the primary actor } \\
\text { wants to achieve. There is a few conventions of naming } \\
\text { use cases, but it is preferable to use active verb phrase in } \\
\text { the use case name. Furthermore, chosen convention } \\
\text { should be used consistently in all us cases. }\end{array}$ \\
\hline \multirow[t]{5}{*}{ Step Level } & $\begin{array}{l}\text { Too Complex } \\
\text { Sentence Structure }\end{array}$ & $\begin{array}{l}\text { The structure of a sentence used for describing each step } \\
\text { of use case should be as simple as possible. It means that } \\
\text { it should generally consists of a subject, a verb, an object } \\
\text { and a prepositional phrase }\end{array}$ \\
\hline & Lack of the Actor & $\begin{array}{l}\text { The reader should know which step is performed by } \\
\text { which actor. Thus, every step in a use case should be an } \\
\text { action that is performed by one particular actor. }\end{array}$ \\
\hline & $\begin{array}{l}\text { Misusing Tenses and } \\
\text { Verb Forms }\end{array}$ & $\begin{array}{l}\text { Use cases should be written in a way which is highly } \\
\text { readable for everyone. Therefore the action ought to be } \\
\text { described from the user point of view. In order to ensure } \\
\text { this approach, the present simple tense and active form of } \\
\text { a verb should be used. }\end{array}$ \\
\hline & $\begin{array}{ll}\text { Using } & \text { Technical } \\
\text { Jargon } & \\
\end{array}$ & $\begin{array}{l}\text { Technical details should be kept outside of the functional } \\
\text { requirements specification. }\end{array}$ \\
\hline & Conditional Steps & $\begin{array}{l}\text { Conditional sentences (if condition then action) is } \\
\text { preferred by computer scientists, but it can confuse the } \\
\text { customer. Especially it can be difficult to read when } \\
\text { nested if statement is used in a use case step. Use cases } \\
\text { should be as readable as possible. Such a style of writing } \\
\text { makes it complex, hard to understand and follow. }\end{array}$ \\
\hline
\end{tabular}


Table 62 - Use Case Checklist of Text2Test (Sinha et al., 2010)

\begin{tabular}{|l|l|}
\hline Condition of interest & Description \\
\hline Stylistic checks & $\begin{array}{l}\text { For English sentences e.g., voice use of actions of recognized kinds, use of } \\
\text { anaphora. }\end{array}$ \\
\hline Complexity checks & $\begin{array}{l}\text { For the number of actions in a statement, the number of statements in a use case, } \\
\text { and so on. }\end{array}$ \\
\hline Completeness checks & Of use case statements e.g., missing actors and actions, missing parameters. \\
\hline Structural checks & For the model e.g., consistent use of aliases, dangling use case references. \\
\hline Flow checks & For data and control flow e.g., attempts to use items before they are created. \\
\hline Concurrency-related checks & e.g., for possibly concurrent actions or possibly non-serializable behaviors. \\
\hline Inter-model checks & $\begin{array}{l}\text { To compare the actors and items referenced in a use case to an associated domain } \\
\text { model. }\end{array}$ \\
\hline
\end{tabular}

Table 63 - Common use case defects (Liu et al., 2014)

\begin{tabular}{|l|l|}
\hline Defect & Description \\
\hline $\begin{array}{l}\text { Inconsistent step numbering } \\
\text { defect }\end{array}$ & $\begin{array}{l}\text { Inconsistent step numbering captures the situation where the sentence numbers of main } \\
\text { flow or alternative flow are not consistent. This may lead to incorrect step referencing. }\end{array}$ \\
\hline $\begin{array}{l}\text { Use case contains the } \\
\text { unclear alternative flow } \\
\text { starting step defect }\end{array}$ & $\begin{array}{l}\text { In some use cases, the starting step (in main flows) of the alternative flow is not clearly } \\
\text { specified. This may lead to ambiguity when merging the alternative flows with the } \\
\text { main flow. }\end{array}$ \\
\hline $\begin{array}{l}\text { Conflict is a function } \\
\text { deciding whether two } \\
\text { predicates conflict }\end{array}$ & $\begin{array}{l}\text { An overly-strong precondition is one such that inconsistencies between the } \\
\text { precondition and the guard conditions of an edge may occur. }\end{array}$ \\
\hline $\begin{array}{l}\text { The use case contains the } \\
\text { missing alternative flow } \\
\text { defect. }\end{array}$ & $\begin{array}{l}\text { Missing of alternative flows is the case when the main flow defines some action under } \\
\text { some specific condition, however not all the other possible conditions are addressed. }\end{array}$ \\
\hline $\begin{array}{l}\text { Missing Scenarios } \\
\text { Missing Pre/Post-conditions }\end{array}$ & $\begin{array}{l}\text { By interacting with the users, the approach must be able to find missing scenarios } \\
\text { which are not captured by the use case documents. } \\
\text { In most of the use cases, the authors of the use case specifications tend to focus on } \\
\text { Consequently the use cases usually have their preconditions and post-conditions } \\
\text { partially documented; missing or redundant conditions also appear frequently. } \\
\text { Therefore, it is extremely helpful to provide a way for the users to correct/complete the } \\
\text { pre-conditions/post-conditions in order to improve the integrity of the use case } \\
\text { document. }\end{array}$ \\
\hline
\end{tabular}

\section{A.2.2. \\ Dynamic Analysis of Software Requirements Specification}

Table 64 - Consistency and Completeness in CMPN (Lee et al.,1998)

\begin{tabular}{|l|l|}
\hline Property & Description \\
\hline Deadlock & $\begin{array}{l}\text { If there exists a set of transitions that are never enabled. This type of flaw is analogous to unreachable } \\
\text { code in programs. Since use cases are expected to reflect genuine needs, it is reasonable to require } \\
\text { that CMPNs do not contain transitions that are never enabled (inconsistency). }\end{array}$ \\
\hline Non-determinism & $\begin{array}{l}\text { If the reachability analysis reveals the presence of non-deterministic execution paths, the CMPN may } \\
\text { be incomplete because users may have forgotten to fully specify the constraints associated with the } \\
\text { use cases. It must be emphasized that nondeterministic execution paths may have been introduced on } \\
\text { purpose and that the final decision can be made only by the domain experts. }\end{array}$ \\
\hline $\begin{array}{l}\text { Missing toggle place } \\
\text { reference }\end{array}$ & $\begin{array}{l}\text { State variables are modeled as toggle places, i.e, a toggle place consists of a pair of places where a } \\
\text { place is the negation of the other. When one of these places is missing, the CMPNs are surely } \\
\text { incomplete. }\end{array}$ \\
\hline $\begin{array}{l}\text { Toggle place values } \\
\text { never modified }\end{array}$ & $\begin{array}{l}\text { State variables are never changed during system operation. CMPNs must contain transitions that are } \\
\text { capable of removing or depositing a token from or to the toggle places, respectively. Otherwise, the } \\
\text { CMPNs are surely incomplete. }\end{array}$ \\
\hline $\begin{array}{l}\text { Slices with no shared } \\
\text { transitions }\end{array}$ & $\begin{array}{l}\text { Slices are likely to contain shared transitions which serve as synchronization points among } \\
\text { concurrently executing CMPN slices. The presence of a slice that never interacts with the rest of the } \\
\text { system is likely, although not conclusively, to be incorrect. }\end{array}$ \\
\hline
\end{tabular}


Table 65 - Faults Detected by Time Petri-Nets (Lee et al., 2001)

\begin{tabular}{|l|l|}
\hline Fault & Description \\
\hline Missing information & $\begin{array}{l}\text { The name of places or transitions in Petri-Net are not specified; } \\
\text { Place, that is not an initial place does not have input arcs; } \\
\text { Place, that is not an final place does not have output arcs; }\end{array}$ \\
\hline Wrong information & $\begin{array}{l}\text { Timing constraints inconsistently - intra-scenario: contradictory timing constraints in a scenario; } \\
\text { Timing constraints inconsistently - inter-scenario: specifying two different values on the same } \\
\text { interval of two events may cause this inconsistency; } \\
\text { Non-determinism: when there is the same event sent from the same object with the same timing } \\
\text { constraints but with different objects to receive this event; } \\
\text { Incorrectly specified timing constraints. }\end{array}$ \\
\hline
\end{tabular}

Table 66 - Use Case Defect Classification (Denger et al., 2005)

\begin{tabular}{|l|l|l|}
\hline Defect Class & Description & Example \\
\hline Incorrectness & $\begin{array}{l}\text { The UC does not match the expected or intended } \\
\text { behavior; that is, the information presents in the UC is } \\
\text { wrong and does not represent the user requirements. }\end{array}$ & $\begin{array}{l}\text { The flow of a UC does not } \\
\text { represent the flow of activities } \\
\text { expected by the user. }\end{array}$ \\
\hline Incompleteness & $\begin{array}{l}\text { The UC does not contain all necessary scenarios. } \\
\text { The UC set does not contain all necessary Use Cases. } \\
\text { Information that is required for subsequent activities is } \\
\text { not present. }\end{array}$ & $\begin{array}{l}\text { An important exception is not } \\
\text { specified, a certain actor is not } \\
\text { considered. }\end{array}$ \\
\hline \multirow{J}{*}{ Inconsistency } & $\begin{array}{l}\text { A piece of information of a single Use Case or of } \\
\text { different Use Cases is described in at least two } \\
\text { different, incompatible ways so that there is a } \\
\text { contradiction between them. }\end{array}$ & $\begin{array}{l}\text { The quality constraints of a Use } \\
\text { Case contradict the event flow. } \\
\text { One user action in two different } \\
\text { Use Cases requires contradictory } \\
\text { system behavior. }\end{array}$ \\
\hline Ambiguity & $\begin{array}{l}\text { Elements of the Use Case can be interpreted in two or } \\
\text { more ways. Thus, it is not clear which of the } \\
\text { interpretations are true. }\end{array}$ & $\begin{array}{l}\text { A condition containing "and" } \\
\text { and "or" does not explicitly state } \\
\text { the required bracketing. }\end{array}$ \\
\hline
\end{tabular}

Table 67 - Properties of UC-LTSs (Sinnig et al., 2009)

\begin{tabular}{|l|l|}
\hline Property & Description \\
\hline Well-formedness & $\begin{array}{l}\text { All use case steps and extensions IDs be unique. } \\
\text { For every step or extension reference, there exists a corresponding use case step or use case } \\
\text { extension within the same use case, respectively. } \\
\text { There are not circular inclusions in include relationships. } \\
\text { The last element of every use case step sequence be either Goto, Success or Failure. }\end{array}$ \\
\hline Livelock & $\begin{array}{l}\text { Phenomenon, where an application performs an infinite sequence of internal actions. } \\
\text { Erroneous use case specifications may contain loops of internal system steps. }\end{array}$ \\
\hline Refinement & $\begin{array}{l}\text { Verify whether a refining use case model has the same traces and exposes the same non- } \\
\text { determinism (existing nondeterministic transitions are preserved). }\end{array}$ \\
\hline
\end{tabular}

Table 68 - Properties of Timed and Controlled Petri-Nets (Zhao and Duan, 2009)

\begin{tabular}{|l|l|}
\hline Property & Description \\
\hline Completeness & $\begin{array}{l}\text { All places and transitions are specified by particular names; } \\
\text { Not isolated subnet exists in the TCPN model of each use case; }\end{array}$ \\
\hline Consistency & $\begin{array}{l}\text { The TCPN model itself is consistency, i.e. the TCPN is live; } \\
\text { TCPN models of related use cases are consistency, i.e. TCPN model of the use case U is } \\
\text { consistent with that of U's include use case; TCPN model of the use case U is consistent with } \\
\text { that of U's base use case. }\end{array}$ \\
\hline Correctness & $\begin{array}{l}\text { The reachability graph of TCPN model is correct; } \\
\text { The TCPN models is bounded; } \\
\text { The time delay of the transition of TCPN models is valid. }\end{array}$ \\
\hline
\end{tabular}

Table 69 - Properties of Reactive Petri-Nets (Somé, 2010)

\begin{tabular}{|l|l|}
\hline Property & Description \\
\hline Balancedness & The absence of connection between parallel place/transitions. \\
\hline 1 -safety & $\begin{array}{l}\text { If for all reachable marking (state) M, each place in Petri-Net contains at most one } \\
\text { token. These two properties are sufficient conditions for the generation of structure- } \\
\text { preserving State- Charts from Petri-Nets }\end{array}$ \\
\hline Lack of non-determinism & The Petri-Nets obtained from use cases are devoid of non-determinism. \\
\hline
\end{tabular}

